\title{
Controlled levels of canonical Wnt signaling are required for neural crest migration
}

\author{
PhD Thesis \\ in partial fulfillment of the requirements \\ for the degree "Doctor rerum naturalium (Dr.rer.nat)" \\ in the Molecular Biology Program \\ at the Georg August University Göttingen \\ Faculty of Biology
}

Submited by

Ewa Anna Maj

Born in Gdańsk, Poland

September 2015 


\section{Affidavit}

Herewith I declare that I prepared the PhD thesis "Controlled levels of canonical Wnt signaling are required for neural crest migration" on my own and with no other sources and aids than quoted.

30.09.2015

Submission date

Ewa Anna Maj 
Members of the Thesis Committee:

\section{Supervisor}

Prof. Dr. Annette Borchers, Molecular Embryology, Philipps-Universität Marburg

Second member of the thesis committee

Prof. Dr. Andreas Wodarz, Anatomy and Molecular Cell Biology, Universität zu Köln

Third member of the thesis committee

Prof. Dr. Heidi Hahn, Molecular Developmental Genetics, Georg-August-Universität 


\section{List of publications}

Maj E, Künneke L, Melchert J, Pieler T, Aspelmeier T and Borchers A. Controlled levels of canonical Wnt signaling are required for neural crest migration. In preparation

Shao L, Fan C, Maj E and Fang Q (2010) Molecular characterization of nonstructural protein NS38 of grass carp reovirus. Virol Sin., 25(2):123-9. 
Tabel of content

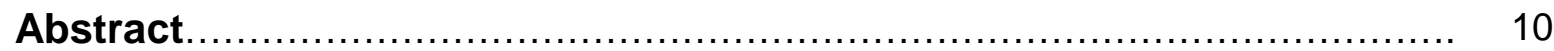

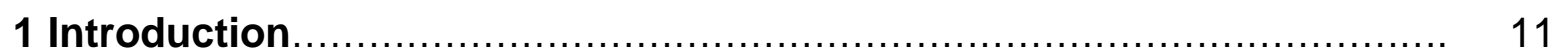

1.1 An overview of NC cell development 11 and the importance of NC research..

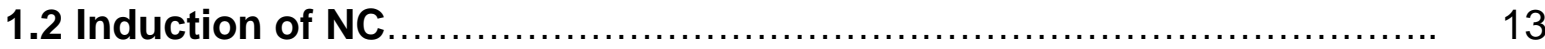

1.2.1 NC induction during gastrulation and neurulation ........................... 13

1.2.2 Molecular basis of NC induction-cross-talk of BMP, FGF and Wnt 15 signaling....

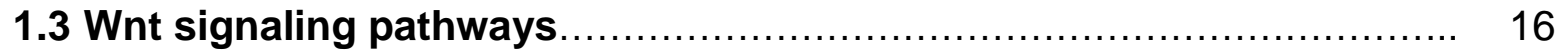

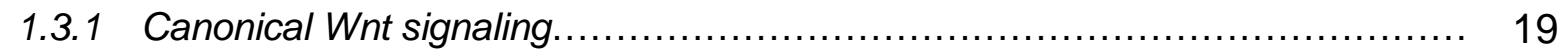

1.3.2 Canonical Wnt signaling in the development of NC cells...................... 20

1.4 Transcriptional network determining NC identity $\ldots \ldots \ldots \ldots \ldots \ldots \ldots \ldots \ldots \ldots \ldots \ldots \ldots \ldots \ldots$

1.5 Epidermal-to-mesenchymal transition (EMT)- basic concept........... 22

1.5.1 Loss of epithelial polarity and modulation of cell adhesion ...................... 23

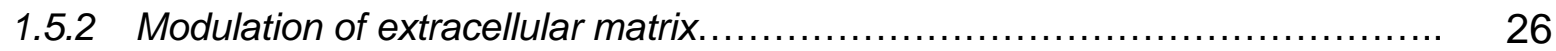

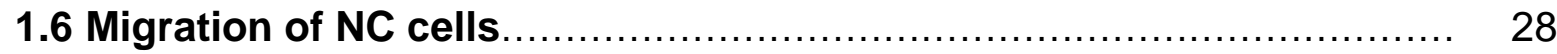

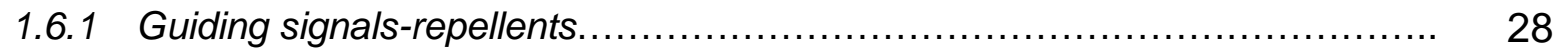

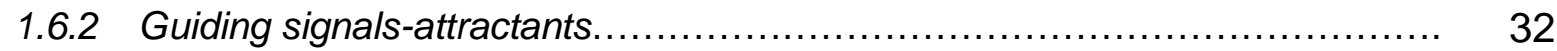

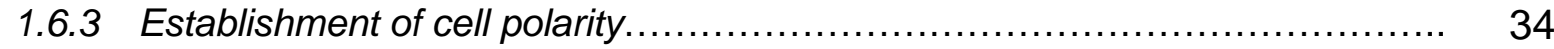

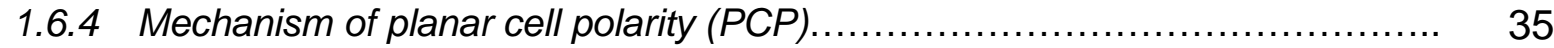

1.6.5 Role of PCP in NC migration ............................................... 41

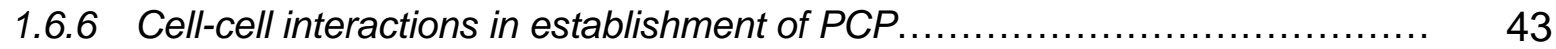

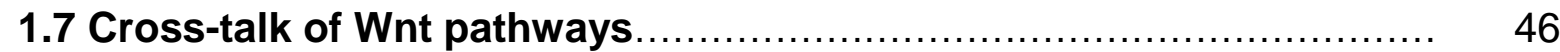

1.8 Methodes to investigate migration of NC cells in Xenopus laevis...... 47

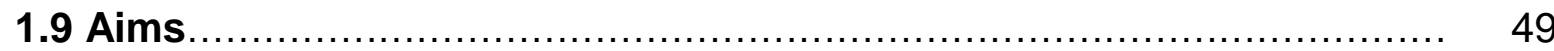




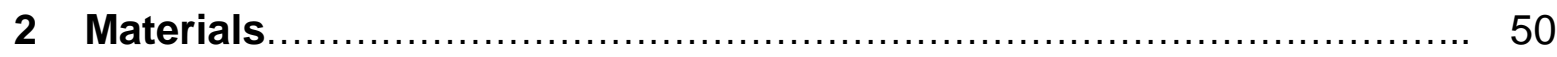

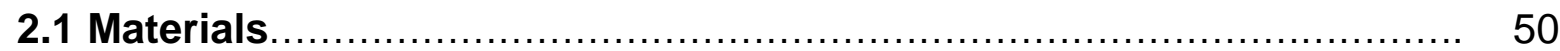

2.2 Chemicals, solutions, media and buffers ................................... 50

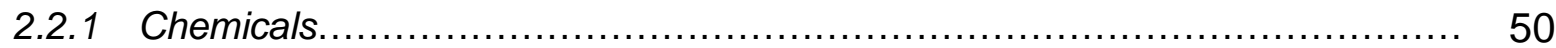

2.2.2 Buffers and Solutions......................................................... 50

2.3 Other chemical substances and reagents.................................. 51

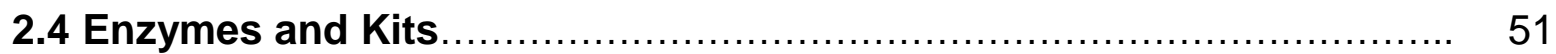

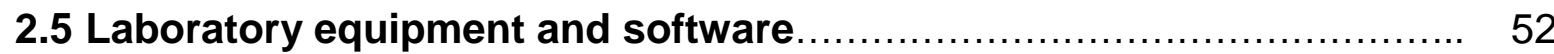

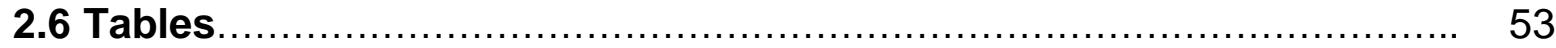

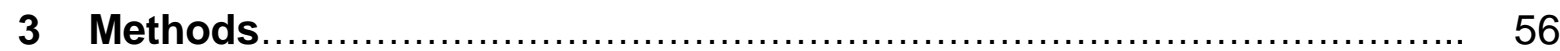

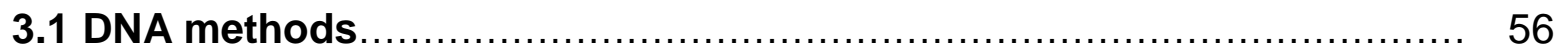

3.1.2 DNA concentration measurement .............................................. 56

3.1.3 Agarose-gel electrophoresis..................................................... 56

3.1.4 DNA restriction digest.............................................................. 57

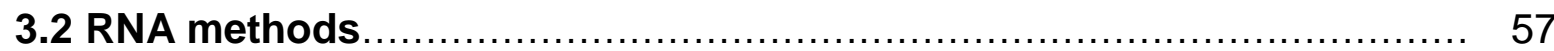

3.2.1 Total RNA extraction, CDNA synthesis and RT-PCR .......................... 57

3.2.2 Revers transcription and RT-PCR ........................................... 58

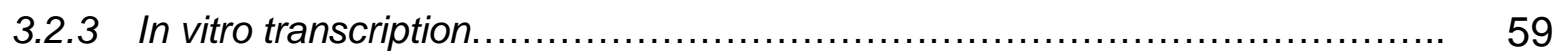

3.2.3.1 In vitro transcription of dioxigenin-labelled antisense RNA for whole mount in 59 situ hybridization (WISH).....

3.2.3.2 In vitro transcription of capped-mRNA for microinjections........................ 59

3.2.4 RNA analysis and concentration measurement ................................. 60 
3.4.1 Preparation of Xenopus laevis testis.............................................. 60

3.4.2 Injections and culture of Xenopus laevis embryos.................................. 60

3.4.2.1 Functionality of GR-inducible constructs in canonical Wnt 61 pathway.

3.4.2.2 In vivo and in vitro analysis of NC migration ..................................... 61

3.4.2.3 Xenopus NC explants for life-cell imaging ........................................ 62

3.4.2.4 ß-catenin localization in Xenopus NC explants............................... 62

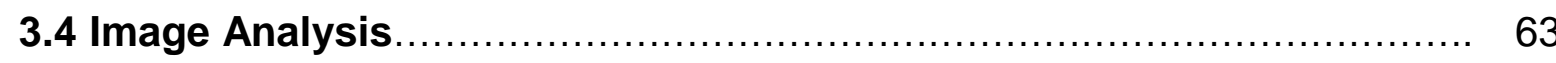

3.4.1 Data evaluation- cell classification ............................................... 64

3.4.2 Data evaluation-mean cluster size .............................................. 65

3.4.3 Data evaluation-mean square displacement ...................................... 65

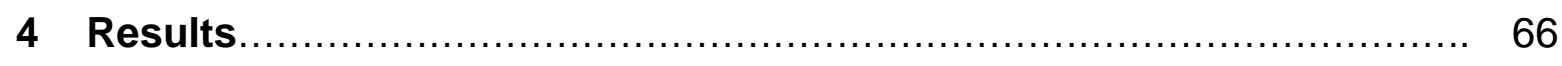

4.1 Activation of canonical Wnt signaling by BIO treatment 66

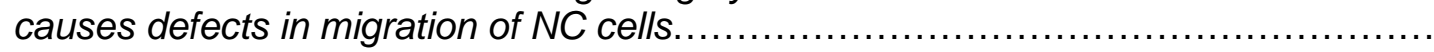

4.2 Functionality of Lef1/Tcf3 constructs in double axis assay ........................... 69

4.3 Activation of Lef1/Tcf3 signaling causes the same 69

defects as $B I O$ treatment.

4.4 Activation of canonical Wnt signaling also causes 72

defects in migration of Sox10-positive NC cells.

4.5 Inhibition of canonical Wnt signaling by IWR-1 treatment causes the same 75 defects in migration of NC cells as BIO treatment.

4.6 Functionality of Lef1/Tcf3 constructs in ventralization assay ........................ 76

4.7 Inhibition of Tcf3 signaling causes the same 76

defects in migration of NC cells as Lef1/Tcf-activation

4.8 Inhibition of canonical Wnt signaling also causes 78

defects in migration of Sox10-positive NC cells. 
4.9 Activation of canonical Wnt signaling by BIO affects in vitro NC migration.

79

4.10 Activation of canonical Wnt signaling by Lef1-GR affects in vitro NC migration..... 80

4.11 Inhibition of canonical Wnt signaling by IWR1 affects in vitro NC migration......... 83

4.12 Inhibition of canonical Wnt signaling by Tcf3 $\triangle C-G R$ affects in vitro NC migration.. 84

4.13 Comparison of Mean Square Displacement................................. 85

4.14 Endogenous nuclear ß-catenin levels decrease during NC migration............... 86

4.15 Modulation of non-canonical Wnt pathway................................. 89

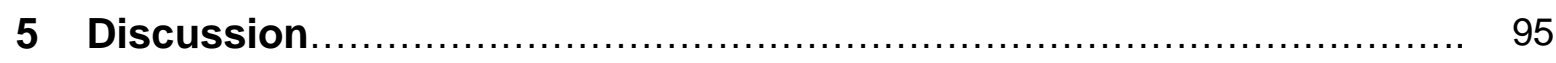

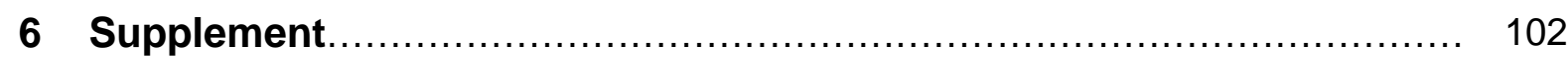

6.1 Functionality of Lef1/Tcf3 constructs in double axis assay ........................ 102

6.2 Functionality of Lef1/Tcf3 constructs in ventralization assay..................... 103

6.3 Inhibition of canonical Wnt pathway by overexpression 104

of EnR-Lef1-GR does not cause any defects in migration of Twist-positive NC cells.....

6.4 Activation of canonical Wnt signaling by LiCl treatment 105

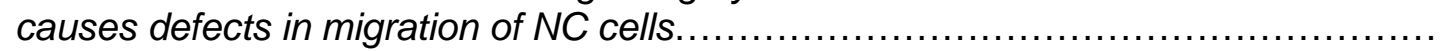

6.5 Modulation of non-canonical Wnt pathway affects migration 106

of Sox10-positive NC cells...

6.6 Determination of Twist and Sox10 expression by RT-PCR ..................... 107

6.7 Determination of c-Jun and Brachury expression by RT-PCR ................. 108

6.8 Effects of chemical modulation on AP2- and Krox20-positive NC cells.............. 109

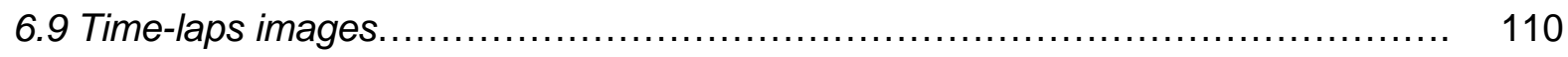

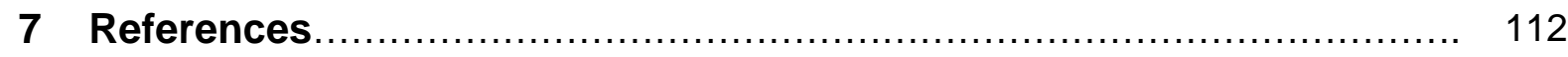

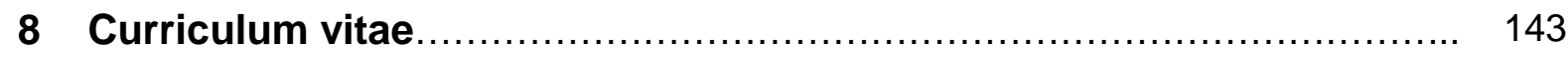




\section{Acknowladgments}

First of all, I would like to thank my supervisor Prof. Borchers for giving me the opportunity to do my $\mathrm{PhD}$ thesis in her laboratory and to work on a very interesting project. I am grateful for all the guidance and help. I would also like to thank Prof. Pieler who kindly agreed on my further work in his Department during 'translocation' of our research group into new laboratory in Marburg

I would also like to thank the members of my Thesis Committee, Prof. Heidi Hahn and Prof. Andreas Wodarz for the helpfull discussions and advices, which I got during our meetings.

Additionally, I would like to thank Dr. Timo Aspelmeier and Lutz Künneke for fruitful collaboration

Furthermore, I want to thank all my colleagues from the Dept. of Molecular Embryology in Marburg and from the Dept. of Developmental Biochemistry in Göttingen, especially Dr. Martina Podleschny, Dr. Peter Wehner, Hanna Berger, Dr. Juliane Melchert, Dr. Juliane Wellner, Dr. Sreemukta Acharya, Dr. Hung-wei Sung and Franziska Winkler for all the technical support.

Last but not least, I would like to thank the International MSc/PhD program in Molecular Biology and especially Dr. Steffen Burkhardt for giving me an opportunity to study in Göttingen.

Finally, I want to thank my family and friends for all the love and care. 


\section{Abstract}

Canonical Wnt signaling has been shown to orchestrate the development of the neural crest (NC), a transient population of multipotent, migratory cells that generate a vast array of cell types. Canonical Wnt signaling is required for NC induction, as well as differentiation; however, its role in NC migration remains elusive. Conversely, ß-catenin independent, non-canonical Wnt pathways have been shown to be required in the migration of NC cells. To delineate a possible function of canonical Wnt-signaling in Xenopus NC migration, canonical Wnt signaling was activated or inhibited at different time points after NC induction using chemical modulators affecting ß-catenin stability and inducible glucocorticoid fusion-constructs of Lef/Tcf transcription factors. Modulation of non-canonical Wnt pathways was performed using chemical inhibitors and provided a comparision to migration defects observed upon dysregulation of canonical Wnt signaling. In each case, alternations in NC migration were analyzed either in vivo using whole mount in situ hybridization or in vitro by life-cell imaging of explanted NC cells. Ectopic activation of canonical Wnt signaling caused predominantly a strong inhibition of cranial NC migration. Less frequently patterning defects of the cranial NC streams were observed. Intriguingly, inhibition of canonical Wnt signaling phenocopies NC migration defects already observed upon activation. Furthermore, modulation of canonical Wnt caused differences in expression of NC-marker genes. Inhibition of non-canonical Wnt signaling results mostly in formation of unstructured NC branches, however, changes in expression of NC-markers were not observed. Additionally, life-cell imaging in combination with biophysical data analysis of explanted NC cells confirmed the in vivo findings and demonstrated that modulation of both canonical and non-canonical Wnt signalings affect cell mobility and the ability to perform single cell migration. Furthermore, it has been shown that endogenous ß-catenin is present in the nucleus at premigratory stages, but starts to be removed in the progress of migration, suggesting that canonical Wnt activity has to decrease to basal levels at the onset of NC migration. Thus, the data confirms necessity of non-canonical Wnt signaling for proper NC cells migration and support the hypothesis that canonical Wnt signaling needs to be tightly controlled to enable migration of NC cells. 


\section{Introduction}

\subsection{An overview of NC cell development and the importance of NC research}

Neural crest (NC) is a population of multipotent cell progenitors found exclusively in vertebrate embryos. They are induced along the entire length of neuraxis as a bilateral, segmented stripe of cells at the lateral border of the neural plate and nonneural ectoderm (Hall, 2008; Le Douarin and Kalcheim, 1999; Sauka-Spengler and Bronner-Fraser, 2008; Steventon et al., 2005). NC cells are subdivided into cranial (Graham et al., 2004; Cordero et al., 2011), cardiac (Kirby et al., 1983; Keyte and Hutson, 2012), vagal (Kuo and Erickson, 2011; Peters-Van Der Sanden et al., 1993; Burns and Le Douarin, 1998; Yntema and Hammond, 1954), trunk (Bronner-Fraser and Fraser, 1989; Serbedzija et al., 1994) and sacral (Burns and Le Douarin, 1998; Anderson et al., 2006) NC cells due to their diversity along neuraxis (Fig.1.1A). Upon closure of the neural plate, NC cells undergo epithelial-to-mesenchymal transition (EMT) (Ahlstrom and Erickson, 2009; Alfandari et al., 2010; Berndt et al., 2008 and Duband, 2010) allowing them to delaminate from prospective neural tube and migrate throughout the embryo (Fig.1.1B). Many of the genes involved in EMT of NC cells are transcription factors which have been also classified as proto-oncogenes contributing to cancer development (Thiery, 2003). Furthermore, motile properties as well as migration behavior of NC cells mirror the migration of cancer cells (Kuriyama and Mayor, 2008; Morales et al., 2005; Theveneau and Mayor, 2012; Dupin and Sommer, 2012). Therefore, investigation of NC migration provides a deeper understanding of the molecular machinery govering the progression and invasivnes of metastatic cancer. Once NC cells reach their final location, they differentiate into various cell types including peripheral and enteric neurons, glia, smooth muscle cells, craniofacial cartilage and bone, endocrine cells and pigment cells (Dupin et al., 2006; Grenier et al., 2009; Hall, 2008; Kirby and Hutson, 2010; Le Douarin and Kalcheim, 1999; Le Douarin and Teillet, 1971; Minoux and Rijli, 2010 and Theveneau and Mayor, 2011a) (Fig.1.1C). Consequently many different systems like skin, face, and heart will have a contribution from the NC cells. Defects in induction, migration or differentiation of NC cell to derivatives will cause severe organ deficienties and malformations. These pathologies, called neurocristopathies (Bolande, 1997; Carmen Carrascosa-Romero and Carlos de Cabo de la Vega, 2012), include disorders such as Waardenburg- 
Shah syndrome (deafness and pigmentation anomalies) (Mahmoudi et al., 2013; Keyte and Hutson, 2012), frontonasal dysplasia (malformations of the craniofacial structures) (Dee et al., 2013) DiGeorge syndrome (craniofacial, heart, kidney and mental defects) (Keyte and Hutson, 2012; Bertsch et al., 2015; Zhang et al., 2014) and Bardet-Biedl syndrome (malformations of the craniofacial structures) (Tobin et al., 2008). Therefore, revealing the mechanisms of NC development represents an important step in understanding the basis of various pathologies.

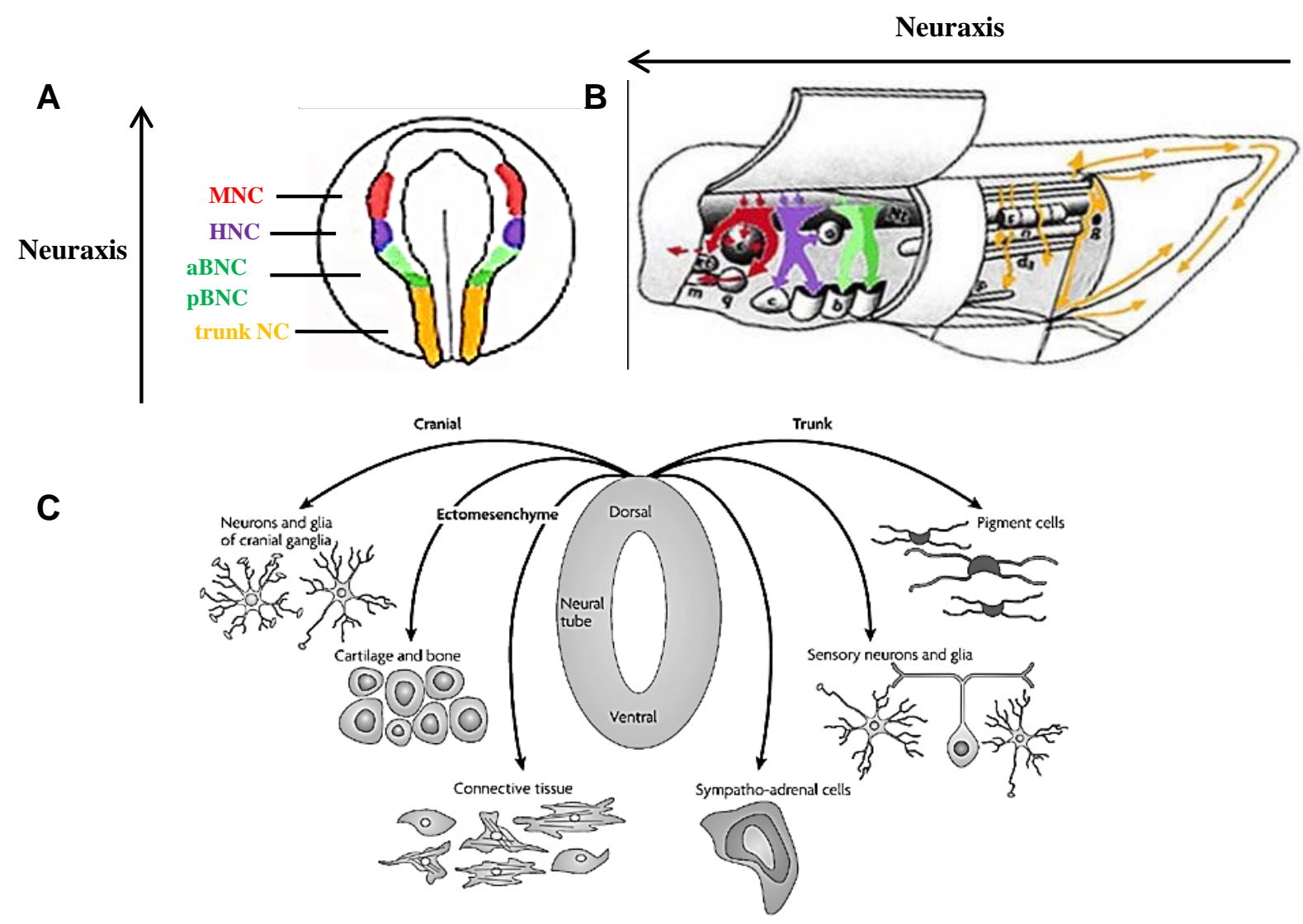

Figure.1.1. Induction, migration and differentiation of the NC cells on example of Xenopus leavis. A NC cells are induced along the whole neuraxis as a segmented group of cells. Cranial NC cells are subdivided into mandibular NC cells (MNC), hyoid NC (HNC), and anterior/posterior branchial NC (aBNC and pBNC). Cardiac, vagal and sacral NC cells being a small subset of cranial and trunk NC are not depicted B Migration of NC cells is performed in streams and quarters them in different locations all over the developing embryo. C Already during migration NC cells initiate a differentiation program which will lead to formation of many various derivatives. Adapted from Mayor et al.,1999 and Mason, 2007. 


\subsection{Induction of NC}

\subsubsection{NC induction during gastrulation and neurulation}

NC induction is a sophisticated and long-lasting process, which in some vertebrates begins already during gastrulation, continues throughout the neurula stages and can be easily monitored by expression of NC-specific genes including several families of transcription factors. During the complex tissues rearrangments of gastrulation and neurulation prospective NC cells are thought to receive variety of inductive signals from different sources, which coordinate the formation of the NC. NC are initially induced already during gastrulation by the signals derived most likely from the ectoderm and subsequently during neurulation by the signals derived from the surrounding paraxial mesoderm, neural plate and non-neural ectoderm (Fig.1.2). Both in Xenopus and zebrafish mesoderm was believed to be the initial source of NC-inductive signals during gastrulation, however, most recent studies show that embryos lacking mesoderm or mesoderm-derived signaling are still able to express NC-specific genes (Wu et al., 2011; Ragland and Raible, 2004). On the contrary, studies in chick embryo show that NC induction during gastrulation can be performed without any support from the surrounding tissues (Basch et al., 2006) leaving the participation of other tissues in initial NC induction during gastrulation as an open question. The final emergence of NC cells at the boarder of neural plate and the nonneural ectoderm into the post-gastrula development raises the question about the contribution of those tissues in the induction of NC cells. There are several lines of evidence that formation of NC cells depends on inductive signals derived from nonneural ectoderm, but also from the underlying paraxial mesoderm (Fig.1.2C). Transplantation experiments using pigmented/non-pigmented axolotl embryos proved that interactions between neural plate and non-neural ectoderm lead to the development of NC in the cells derived from both tissues. (Moury and Jacobson, 1989). Additionally, it has been shown that in vitro recombination of explanted neural plate and non-neural ectoderm from Xenopus and avian embryos is sufficient to activate expression of NC-specific gene Snail2 in the cells derived from both tissues (Mancilla and Mayor, 1996; Selleck and Bronner-Fraser, 1995; Dickinson et al., 1995; Mayor et al., 1995; Nieto et al., 1994). The preliminary evidence for a role of mesoderm as source of inductive signals comes from the path-breaking experiment of Raven and Kloos who were able to obtain NC derivatives in salamander embryos 
by grafting paraxial and lateral mesoderm into the ectoderm of blastocoel (Raven and Kloos, 1945). Further studies show that removal of paraxial mesoderm in Xenopus embryos causes reduced expression of Snail2 and recombination of ectoderm explants with paraxial mesoderm can activate Snail2 expression and induce melanocyte formation (Bonstein et al., 1998, Marchant et al., 1998). Taken together these data provide the support for the necessity of inductive interactions between neural plate, non-neural ectoderm and paraxial mesoderm for proper formation of NC cells.

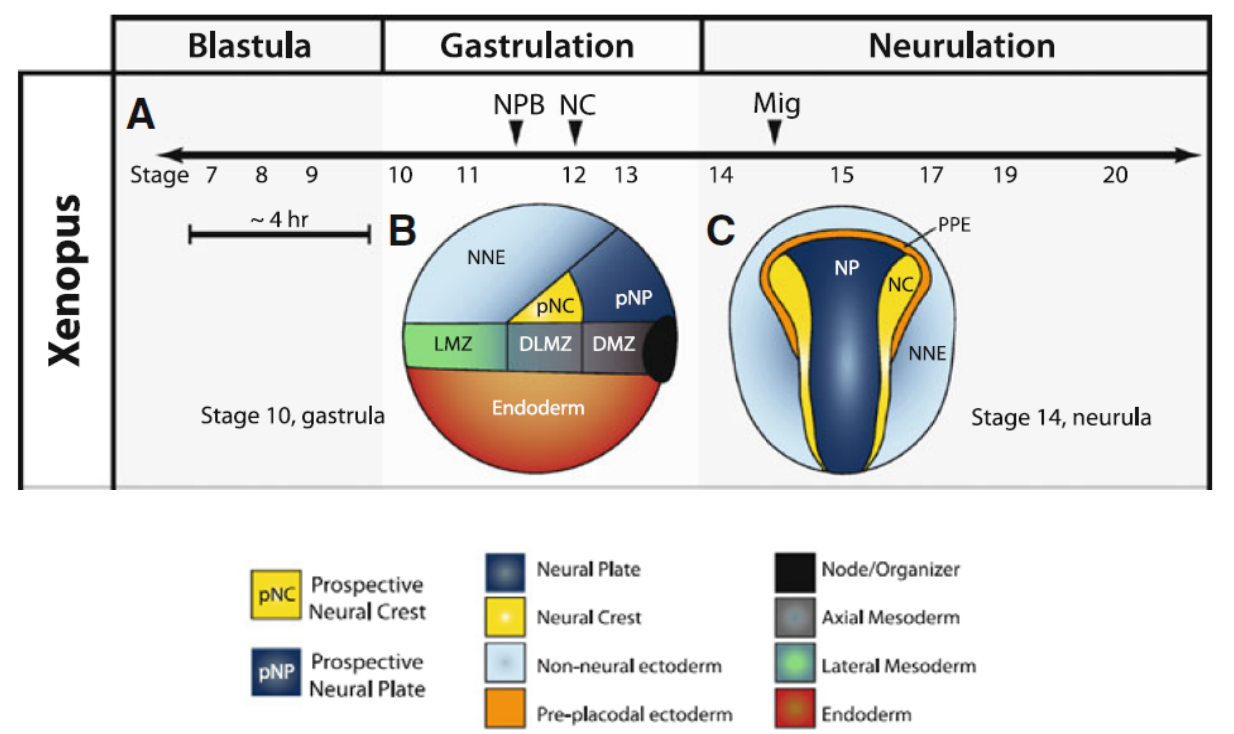

D Neural plate border Neural plate Non-neural ectoderm

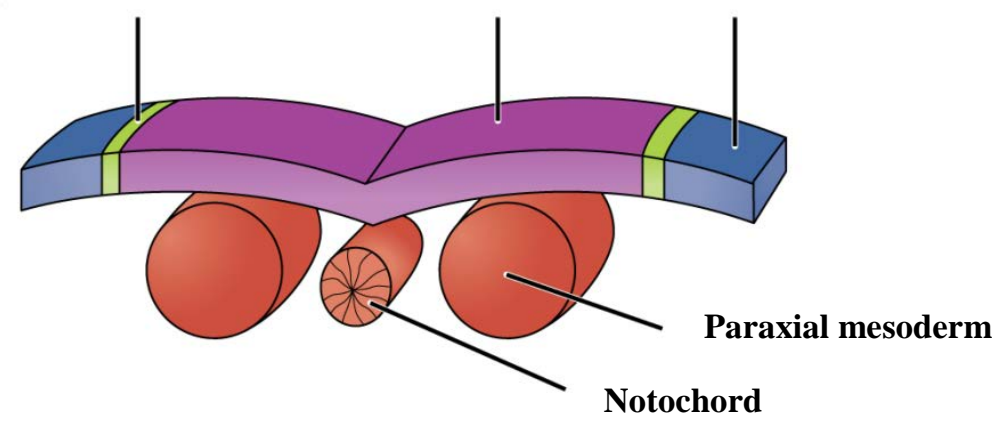

Figure.1.2. Formation of NC cells during gastrulation and neurulation on the example of a Xenopus embryo. A Induction of NC cells in respect to Nieuwkoop and Faber stages of Xenopus development. B Lateral view of the early gastrula. Preliminary NC induction starts at gastrulation. NC-specific markers (NC) are already expressed during gastrulation. Prospective NC cells are found above dorsolateral marginal zone (DLMZ) constituted from prospective paraxial mesoderm. C Dorsal view of the early neurula. NC cells are positioned between neural plate (NP) and non-neural ectoderm (NNE). D The proper induction of NC cells depends on the inductive signals derived from surrounding neural and non-neural ectoderm, but also from the underlying paraxial mesoderm. Combined action of those signals contributes to the formation of neural plate borders (NPB) - a NC progenitor domain. Adapted from Stuhlmiller and Garcia-Castro, 2012; OpenStax College- Antomy and Physiology. 


\subsubsection{Molecular basis of NC induction- cross-talk of BMP, FGF and Wnt signaling}

The molecular machinery controlling formation of NC is still not fully understood, however, series of studies performed across Xenopus, zebrafish, chick and mouse confirm BMP, Wnt and FGF signaling pathways as key players responsible for establishing a signaling network orchestrating the formation of neural plate borders (NPB), a NC progenitor domain which differs from neural plate and non-neural ectoderm (Fig.1.2.2). BMPs are members of the transforming growth factor- $ß$ (TGFß) family of secreted proteins, which binds to type I and type II serine-threonine receptor and transduce the signal through canonical Smad and non-Smad pathways. The BMP signaling pathway has a broad spectrum of biological activities throughout embryonic development and its role in NC induction is tightly linked with the induction of the neural plate. BMP signaling plays a crucial role in the establishment of dorsal-ventral polarity in the early embryo and the promotion of non-neural ectoderm over neural cell fates (Barth et al., 1999). Currently there are two models which explain the role of BMP as well as FGF/Wnt signaling in induction of the NC cells; BMP gradient hypothesis and two-step model. In BMP gradient hypothesis, during gastrulation signaling molecules including noggin, chordin and follistatin, which are derived from the dorsal mesoderm induce the formation of the neural plate by blocking BMPs signaling in the ectoderm (Hammerschmidt et al., 1996). As a consequence of a spatial concentration gradient at neurulation, regions with high levels of BMP signaling form non-neural ectoderm while an intermediate level of BMP signaling together with FGFMnt signaling from the adjacent tissues determine NC fate at the neural plate border (NPB) - a NC progenitor domain (Mayor et al., 1995; Marchant et al., 1998; Tribulo et al., 2003; Weinstein and Hemmati-Brivanlou, 1999; Morgan and Sargent, 1997; Nguyen et al., 1998). On the contrary, various studies contradict the gradient hypothesis and propose a two-step model in which inhibition of BMPs during gastrulation allows FGF/Wnt signaling pathways from the adjacent tissues to specify NC, while later activation of BMPs at neurula stages maintains NC fate in NPB (Fig.1.2.2). An alternated absence of Smad1/5/8 signaling activity during gastrulation followed by gradual increase during neurulation at NPB and non-neural ectoderm has been observed in the avian embryos (Sthulmiller and Garcia-Castrio, 2012). Blocking Smad signaling in the chick embryo leads to the loss of NPB-specific genes (Faure et 
al., 2002). Moreover, cultivation of prospective NC explants from gastrulating chick embryos in medium containing Noggin for the first $10 \mathrm{~h}$ has no effect on NC fate, but treatment after $10 \mathrm{~h}$ causes a loss of already present NC-specific genes and promotes induction of neural-specific genes. Correspondingly, cultivation of prospective NC explants with Bmp4 for the first 10h causes loss of NC-specific genes and promotion of epidermal fate, while treatment after first $10 \mathrm{~h}$ causes no change in expression of NC-specific genes (Patthey et al., 2009).

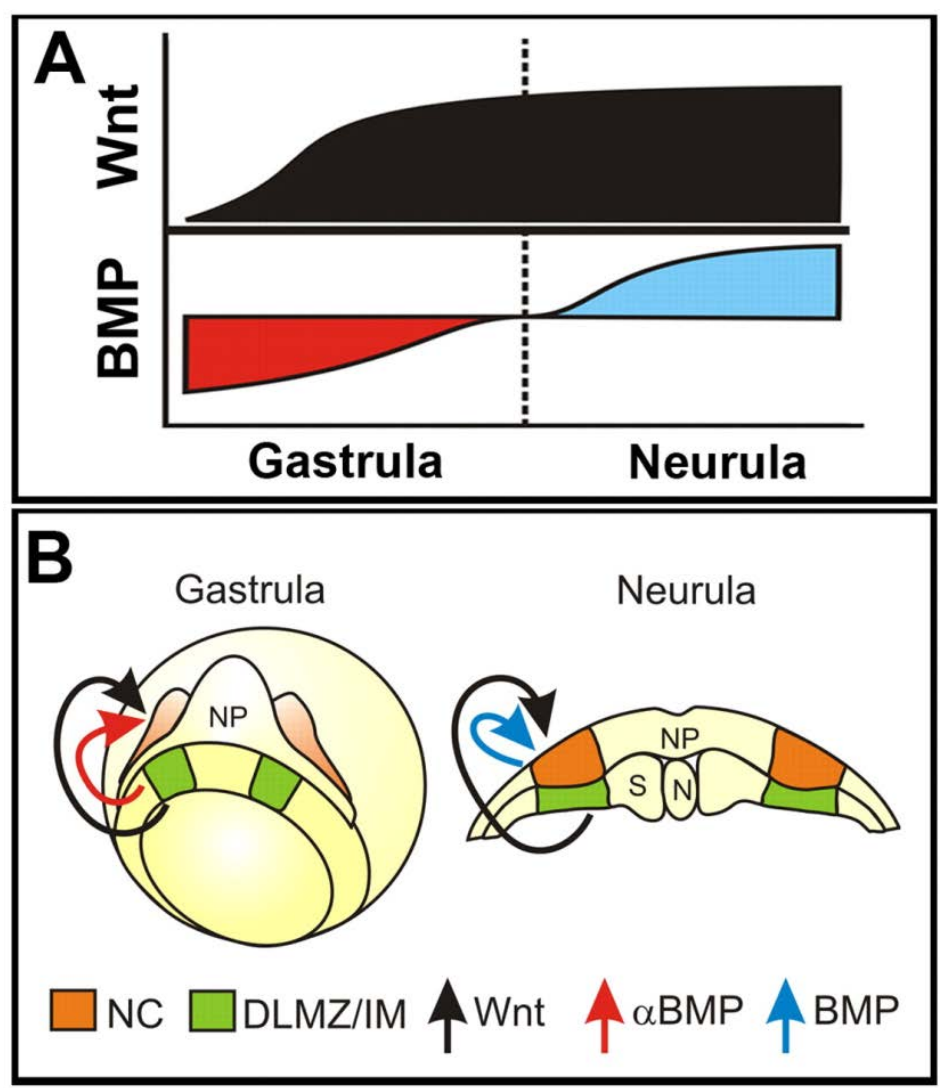

Figure.1.2.2. Two phases of BMP activity during NC induction in Xenopus. During gastrulation initial induction of NC cells requires inhibition of BMP signaling, while later during neurulation BMP signaling becomes activated. Wnt signaling is required in both steps of NC induction. Signals responsible for induction of NC cells are derived from adjacent tissues. Abbreviations: NC-neural crest, DLZM/IM-dorsolateral marginal zone/intermediate mesoderm. Adapted from Steventon et al., 2009.

\subsection{Wnt signaling pathways}

Wnts are a large family of secreted lipid-modified glycoproteins (Willert and Nusse, 2012), which can transduce signal through several different pathways including the canonical or $\beta$-catenin-dependent pathway and the non-canonical or $\beta$-catenin- 
independent pathways, which can be divided further into the Planar Cell Polarity (PCP) pathway and the Wnt/Ca2+ pathway (Komiya and Habas, 2008; Rao and Kühl, 2010) (Fig.1.3). Depending which pathway becomes activated Wnts are subdivided into two classes; canonical and non-canonical according to the ability to induce accumulation of $\beta$-catenin in the nucleus. Signaling through some Wnts such as; Wnt1 (Hinck et al., 1994; Young et al., 1998; Shimizu et al., 1997), Wnt3a (Shimizu et al., 1997) and Wnt8 (Wikramanayake et al., 2004; Kohn and Moon, 2005) has been shown to correlate with accumulation of $\beta$-catenin in the nucleus and transcriptional changes, while signaling trough others like Wnt5a (Shimizu et al., 1997; Kikuchi et al., 2012) and Wnt11 (Kohn and Moon, 2005; Tada and Smith, 2000; Pandur et al., 2002) is $\beta$-catenin-independent and involves regulation of cytoskeleton or calcium release. All the metazoan species express $W n t$ genes and all of those genes encode secreted proteins based on their amino acid structure and biochemical characterization (Coudreuse and Korswagen, 2007; Smolich et al., 1993). Upon translation and targeting to the extracellular space, Wnt proteins are exposed to various modifications, which are essential for folding, secretion and Wnt-signaling activity (Mikels and Nusse, 2006; Coudreuse and Korswagen, 2007; Willert and Nusse, 2012). The most prominent modifications are glycosylation and acylation (Komekado et al., 2007; Kurayoshi et al., 2007; Janda et al., 2012; Takada et al., 2006). Although the mechanism by which the lipid residues are attached to the Wntpolypepide backbone is still not fully understood several comperative and loss-offunction studies suggest the involvement of the Porcupine (Porcn), an ER resident protein in this process (van den Heuvel et al., 1993; Kadowaki et al., 1996; Hofmann, 2000; Barrott et al., 2011; Biechele et al., 2011). Beginning with Wnt secretion, Wntdependent signal transduction requires binding of the Wnt ligand to the extracellular cysteine-rich domain of the seven-transmembrane-span receptor Frizzled (Fz) (Vinson et al., 1989; Adler et al., 1990; Park et al., 1994; Wang et al., 1996) and activation of the cytoplasmic phosphoprotein Disheveled (Dsh) (Wallingford and Habas, 2005), which regulates and branches all of the Wnt pathways (Miller et al., 1999; Komiya and Habas, 2008) (Fig.1.3). Dsh itself is a protein exhibiting a typical modular structure (Fig.1.3). It contains three domains; a DIX, a PDZ and a DEP domain. DIX (Disheveled/Axin) domain located at the N-terminus (Capelluto et al., 2002) is responsible for interaction with a scaffolding protein Axin and functions exclusively in canonical Wnt signaling (Axelrod et al., 1998; Boutros et al., 1998; 
Moriguchi et al., 1999; Penton et al., 2002; Rothbächer et al., 2000). PZD (PSD-95, DLG, ZO1) domain located in the center mediates protein-protein interactions (Cheyette et al., 2002; Wong et al., 2003) and is required for all branches of Wnt signaling (Axelrod et al., 1998; Boutros et al., 1998; Moriguchi et al., 1999; Penton et al., 2002; Rothbächer et al., 2000). Finally, the DEP (Disheveled, EGL-10, Pleckstrin) domian situated at the C-terminus is essential for PCP signaling (Heisenberg et al., 2000; Moriguchi et al., 1999; Tada and Smith, 2000; Wallingford et al., 2000). Additionally, PDZ and DEP domains are used in Wnt/Ca+ signaling (Sheldahl et al., 2003).

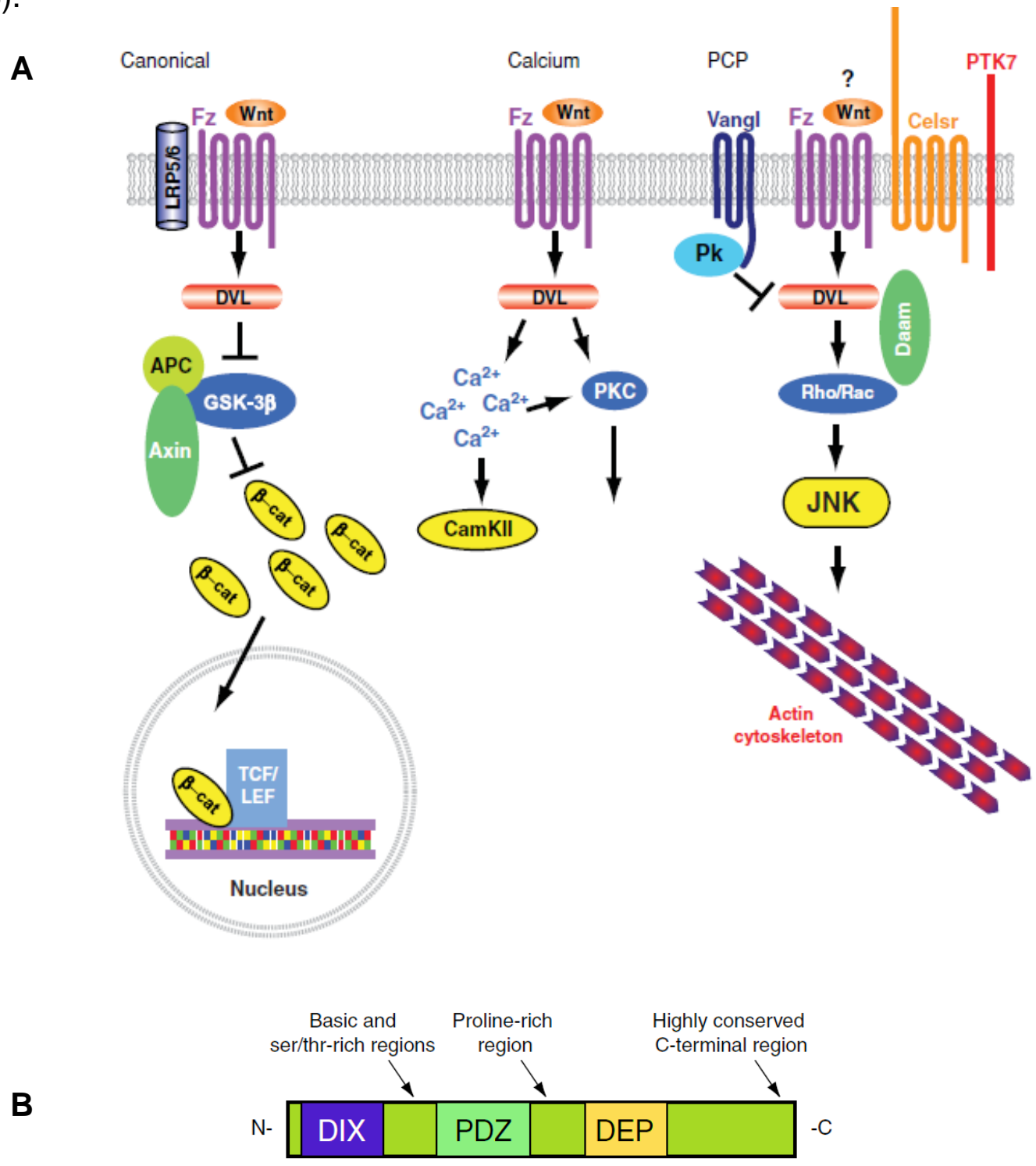

Figure.1.3. Different branches of Wnt signaling pathways. A Canonical WNT/ß-catenin-dependent signaling. B Wnt/Ca+ signaling. C Planar Cell Polarity signaling. B The schematic structure of the Disheveled protein. Disheveled is composed of three major domains; DIX, PDZ and DEP and various conserved motifs including a basic ser/thr-rich region, a proline-rich region and a highly conserved C-terminal region. Adapted from Wallingford and Habas, 2005; Montcouquiol et al., 2006. 


\subsubsection{Canonical Wnt signaling}

The canonical Wnt pathway was identified for the first time in the fruit fly Drosophila melanogaster and intensive studies in the various model organisms delineated the basic molecular signaling framework. Under unstimulated conditions cytoplasmic level of ß-catenin is kept low through the dual phosphorylation (Liu et al., 2002) by glycogen synthase kinase $3 \beta$ (GSK-3ß) (Hart et al., 1999; Yost et al., 1996) and casein kinase 1 $\alpha$ (CK1 $\alpha$ ) (Price, 2006; Amit et al., 2002), which together with the adenomatous polyposis coli tumor suppressor protein (APC) (Hart et al., 1999; Ha et al., 2004), protein phosphatase 2A (PP2A) (Hsu et al., 1999; Seeling et al., 1999; Ratcliffe et al., 2000; Yamamoto et al., 2001) and Axin (Hart et al., 1999; Ikeda et al., 1998; Dajani et al., 2003) form a destruction complex (Stamos and Weis, 2013). Phosphorylated $\beta$-catenin becomes ubiquitylated by $\beta$-TrCP and is targeted for degradation (Aberle et al., 1997; Orford et al., 1997; Kitagawa et al., 1999; Latres et al., 1999; Liu et al., 1999) (Fig.1.3.1A). Upon binding of canonical Wnt ligands to their coreceptors Frizzleds ( $\mathrm{Fz}$ ) and low-density-lipoprotein-related protein5/6 (Lrp5/6) on the cell surface (He et al., 2004; MacDonald and He, 2012) Lrp5/6 becomes dually phosphorylated by $\mathrm{CK} 1$ and GSK-3 $\beta$ and inactivates the destruction complex by recruitment of Axin to the plasma membrane (Davidson et al., 2005; Zeng et al., 2005). Additionally, Dsh is also recruited to the plasma membrane where it binds Fz receptors (Wong et al., 2003) and regulates phosphorylation of Lrp5/6 (Bilic et al., 2007). $\beta$-catenin, which is no longer phosphorylated and tagged for degradation translocates to the nucleus and regulates gene expression by interactions with Lef/Tcf family of transcription factors (lymphoid enhancer factor $/ T$ cell factor) (Behrens et al., 1996; Molenaar et al., 1996; van de Wetering et al., 1997). (Fig.1.3.1B). In the absence of Wnt signal Lef/Tcf acts as transcriptional repressors of Wnt target genes by forming a complex with several corepressors including myeloid translocation gene related-1 (Mtgr1) (Moore et al., 2008), corepressor of Pan (Coop) (Song et al., 2010), hydrogen peroxide-inducible clone (HIC5) (Ghogomu et al., 2006; Li et al., 2011) and the most intensively studied Groucho/transducin-like enhancer of split (Gro/TLE) repressor family (Roose et al., 1998; Chen and Courey, 2000). Upon translocation of $\beta$-catenin to nucleus Lef/Tcf factors become converted into transcriptional activators and perform expression of the target genes (Daniels and Weis, 2005). 

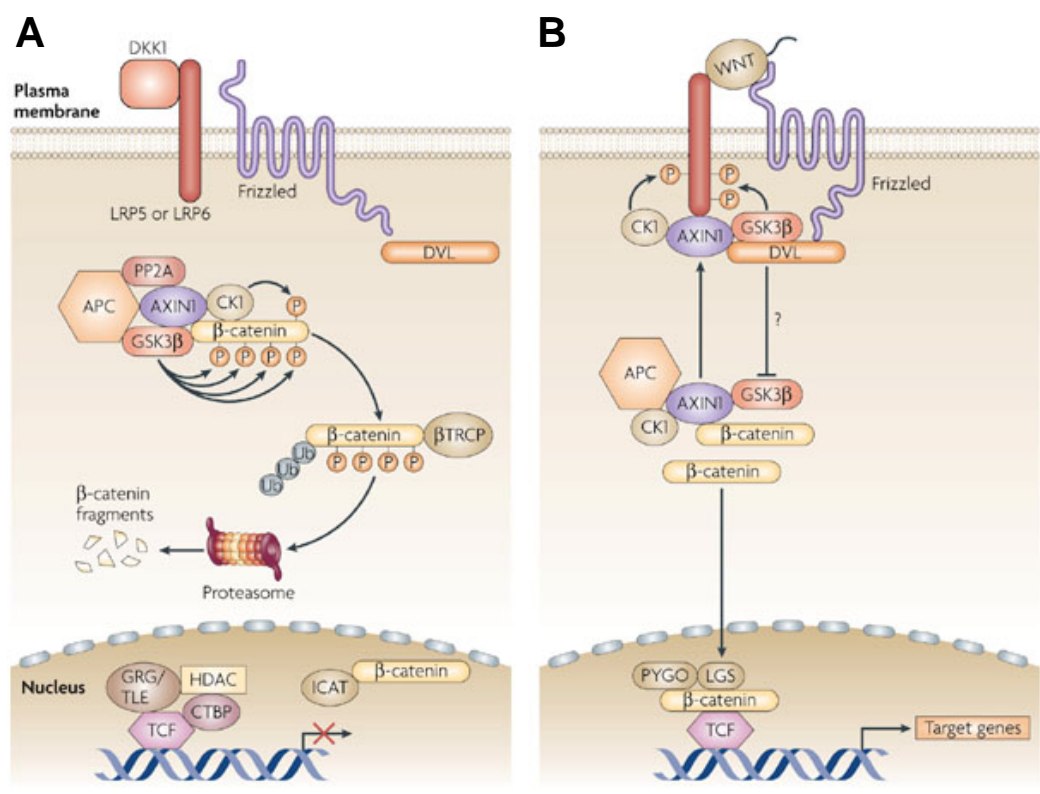

Nature Reviews | Immunology

Figure.1.3.1. Wnt signaling pathway. A In the absence of the Wnt ligands the cytoplasmic and nuclear levels of ß-catenin are kept low. ß-catenin is constantly phosphorylated and tagged for degradation by combined action of proteins forming the destruction complex (APC, PP2A, AXIN1, CK1, and GSK3ß) and BTRCP. B Binding of Wnt ligands cause the disassembly of the destruction complex and translocation of ß-catenin to the nucleus. Adapted from Staal et al., 2008.

\subsubsection{Canonical Wnt signaling in development of NC cells}

As in the case of BMP signaling, broad range of experimental data confirms involvement of canonical Wnt signaling in the induction as well as in delamination and differentiation of NC cells. Inhibition of Wnt signaling in Xenopus and chick embryos by targeting different components of canonical Wnt pathway was shown to impair formation of the NC, while overexpression of some Wnt ligands or downstream components led to ectopic expression of NC-specific genes. Inhibition of canonical Wnt signaling by dominant-negative Wnt constructs results in an inhibition of NC formation in chick and Xenopus embryos (Garcia-Castro et al., 2002; LaBonne and Bronner-Fraser, 1998). Similar effects were also seen by Morpholino-mediated knockdown of $\beta$-catenin, LRP6 or frizzled3 in Xenopus embryos (Deardorff et al., 2001; Hassler et al., 2007; Wu et al., 2005). Correspondingly, activation of canonical Wnt signaling by overexpression of $\beta$-catenin, LRP6, frizzled3 or canonical Wnt ligands in various experimental models led to an expansion of the NC cell population (Chang and Hemmati-Brivanlou, 1998; Deardorff et al., 2001; Saint-Jeannet et al., 
1997; Tamai et al., 2000). In accordance with two-step induction model, canonical Wnt signaling was shown to be required for BMP activation during second step of NC induction in chick embryo (Patthey et al., 2009). What's more, using avian embryos it was shown that canonical Wnt signaling stimulates G1/S transition and activates NC delamination, a process required for initiation of NC migration (Burstyn-Cohen et al., 2004). Finally, a large body of evidence shows that canonical Wnt signaling regulates the last stage of NC development, namely lineage diversification. Constitutive activation of $\beta$-catenin in NC cells promotes sensory neural fate at the expense of other NC derivatives in mice or isolated NC stem cells (Lee et al., 2004). In zebrafish, targeting overexpression of $\beta$-catenin to premigratory NC cells promotes pigment cell formation at the expense of neurons and glia. Conversely, the inhibition of canonical Wnt signaling by truncated Tcf3 or a dominant negative Wnt promoted neuronal fates at the expense of pigment cells (Dorsky et al., 1998). MITF, a transcription factor required for melanocyte development, is directly activated by Wnt signaling indicating that canonical Wnt signaling can directly specify NC fate (Dorsky et al., 2000). As loss of Wnt1 and Wnt3a in the mouse leads to a marked deficiency in NC derivatives, canonical Wnt signaling may also be important for the proliferation of NC cells (Ikeya et al., 1997). Thus, canonical Wnt signaling possibly has a broad range of functions in post-induction stages of NC development.

\subsection{Transcriptional network maintaining NC identity}

Taken together BMBs, Wnts and FGFs signaling pathways integrate into complex transcriptional network, which induce the expression of the first set of regulatory transcription factors called neural plate border specifiers including: Tfap2, Msx1, Zic1, Gbx2, Pax3/7, Dlx5/6, Gata2/3, Foxi1/2 and Hairy2 (Simões-Costa Bronner, 2015) (Fig.1.4A). Those genes specify the neural plate border, a wider domain including prospective epidermis and neural plate. Subsequently these factors along with combinations of the same signaling pathways then trigger the expression of $N C$ specifires, a second set of transcription factors including Ets1, Snail1/2, FoxD3, Sox9/10, Twist, cMyc, and Ap2 (Simões-Costa Bronner, 2015), which activate the epithelial-mesenchymal transition (EMT) allowing NC cells to delaminate from the neural tube and become migratory cells (Fig.1.4B). On the contrary to neural plate border specifiers, expression of $N C$ specifires is restricted exclusively to the 
prospective NC cells. The combination of these genes is most probably also crucial for the maintenance of NC cells in undifferentiated state through delamination and migration (Fig.1.4C,D).

A) NBP specification

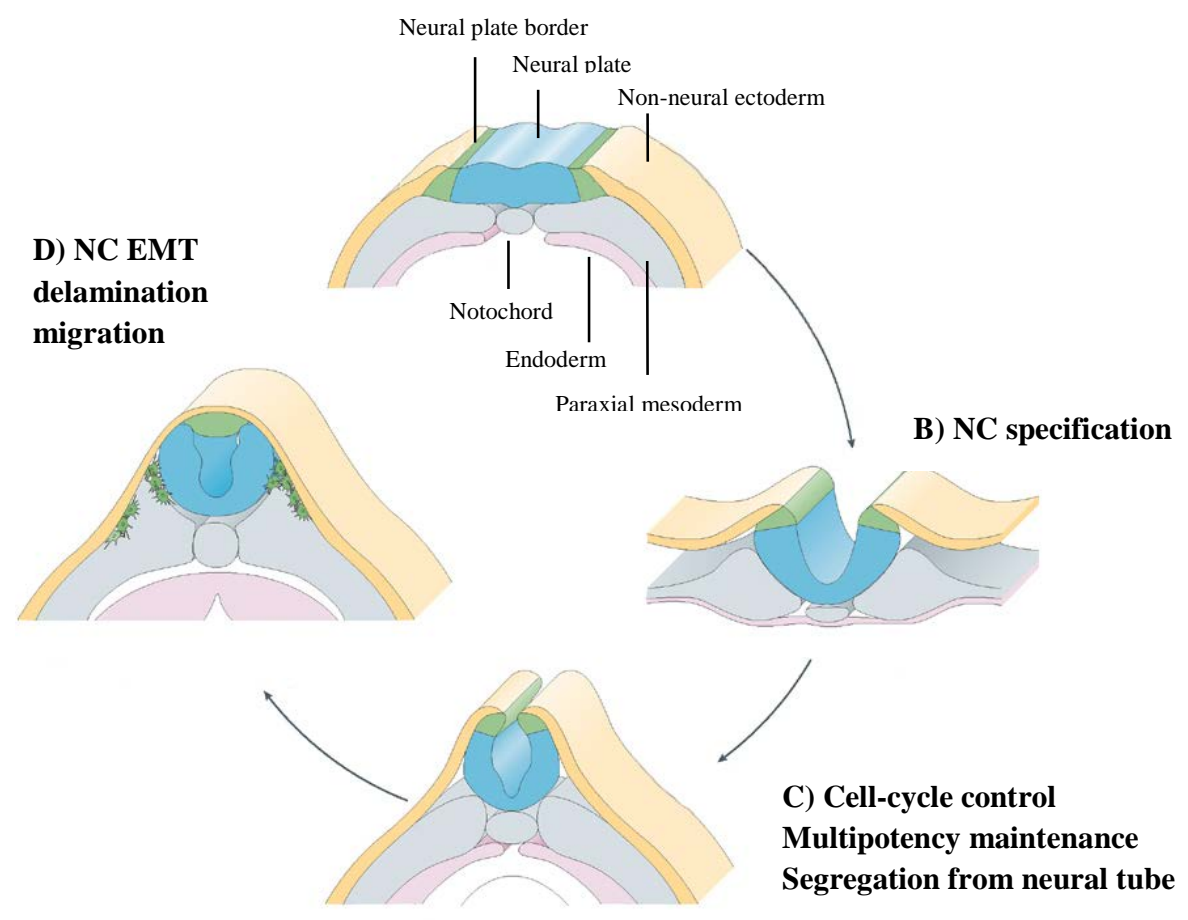

Figure.1.4. Signaling cascades in particular stages of NC induction. A FGF signaling from the paraxial mesoderm as well as canonical Wnt signaling from the paraxial mesoderm and non-neural ectoderm induce expression of NPB specifiers. B FGF/Wnt signaling in combination with NPB specifiers induce expression of NC speifiers which control $\mathbf{C}$ NC maintenance and $\mathbf{D}$ progression through EMT. Adapted from Sauka-Spengler and Bronner-Fraser, 2008.

\subsection{Epidermal-to-mesenchymal transition (EMT)- basic concept}

After specification NC cells undergo epidermal-to-mesenchymal transition (EMT), a sophisticated process, which governs a structural remodeling of the premigratory NC cells. The core of EMT is the breakdown of the highly integrated epithelium and formation of the mesenchymal cells causing complex tissue rearrangements common in early embryogenesis and tumor metastasis (Fig.1.5) (Nieto, 2011; Thiery et al., 2009). During EMT cells can go through the series of transient stages, from typical epithelial tissues with firm cell-cell adhesions, to not fully determined mesenchymal cells with transient contacts and finally to the entirely separated mesenchymal cell without epithelial polarity and cell-cell adhesions (Acloque et al., 2009; Ahlstrom and 
Erickson, 2009; Baum et al., 2008; Duband, 2010; Theveneau and Mayor, 2012). During NC delamination from the prospective neural tube, NC cells undergo partial EMT and migrate as group of mesenchymal cells with transient cell-cell adhesions. EMT of NC cells involves divers cellular processes and is performed in several steps to rearrange cytoskeleton and loosen the stable junctions before delamination from the neuroepithelium. Additionally, EMT is triggered by the combined action of extracellular signals, including components of the extracellular matrix (ECM), like collage and hyaluronic acid, many of secreted ligands, such as members of TGFß, Wnt and FGF family (Vallin et al., 2001; Yook et al., 2006; Thiery and Sleeman, 2006) and numerous transcription factors belonging to the group of NC-specifires. Proteins of the tight junctions responsible for establishing an epithelial state as well as type I classical cadherins are mostly downregulated. Cell-cell adhesions after NC delamination are maintained usually by type II classical cadherins (Kuriyama et al., 2014; Theveneau et al., 2010).

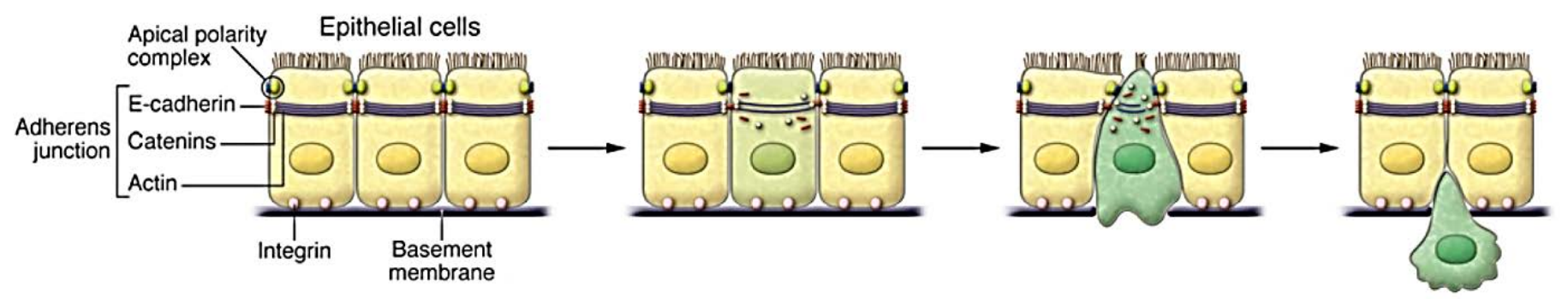

Figure.1.5. Scheme of epidermal-to-mesenchymal transition. Upon EMT NC cells lose stable junction and the typical close-adherent epithelial arrangement. Cells acquire a mesenchymal character, become loosely associated and can easily migrate. Adapted from Acloque et al., 2009.

\subsubsection{Loss of epithelial polarity and modulation of cell adhesion}

Initially, premigratory NC cells are arranged in epithelial structure defined by apicalbasal polarity, which needs to be abolished to allow for the proper delamination (Fig.1.5.1). In avian embryos shortly before migration de-epithelization of cranial NC tissue is achieved by downregulation of claudin-1, which leads to the decomposition of tight junctions and subsequently loss of the apical zones (Aaku-Saraste et al., 1996). Moreover, studies in cultured mouse epithelia show that repression of occludin and claudins (claudin-3, claudin-4 and claudin-7) is performed by the direct interaction of Snail1 (Ikenouchi, 2003), what indicates that similar mechanism might 
be involved in the EMT of NC cells. Disassembly of tight junctions has been recognized as a transition into the gap junctions both in avian and amphibian EMT during early embryonic development (Shook and Keller, 2003). As a support for this observation an excess of the gap junction protein connexin-43a1 (Cx43a1) has been shown to significantly increase the migration rate of mouse cardiac NC cells. On the other hand, downregulation of $\mathrm{C} \times 43 \alpha 1$ impairs migration of human glioblastoma cells, which share similarities with migrating NC cells (Huang et al., 1998). The cadherindependent adhesion, a major player of cell interactions is also subjected to modifications. Cadherins constitute a large family of cell-cell adhesion proteins comprising classical cadherins, protocadherins and atypical cadherins (Halbleib and Nelson, 2006). In various organisms delamination of the cranial neural crest is preceded by a switch in expression between different types of classical cadherins, which bind each other in Ca2+-dependent manner to form adherens junctions (DeLuca et al., 1999). Type I cadherins mediating stronger cell-cell interations (Ecadherin and $\mathrm{N}$-cadherin) are downregulated at the expense of type II cadherins (cadherin-6B, cadherin-7, cadherin-11), which not only mediate weaker, more suitable for migratory NC cell-cell interactions, but also promote protrusive activities (Fig.1.5.1) (Kashef et al., 2009). Modulation of cadherin composition is an essential step in EMT progression, however, there are crucial species-specific differences in the term of cadherin type exchange. Many NC specifires have been shown to directly down- or upregulate specific cadherins with the predominant emphasis on Snail2, Sox10, FoxD3, Twist and Zeb2 (Sip1) transcription factors. Snail2, has been shown to participates in EMT progression in many system ranging from cancer lines to embryos (Niesto et al., 1994; Blanco et al., 2007). The role of Snail2 in the EMT of chick trunk NC cells is linked with the transcriptional repression of $\mathrm{N}$-cadherins by interaction with LIM domain only protein 4 (Lmo4) (Ferronha et al., 2013). On the contrary, in Xenopus $\mathrm{N}$-cadherins are only slightly downregulated during delamination and migration of cranial NC cells and have been shown to be crucial for the response to chemoattractants (Barriga et al., 2013; Theveneau et al., 2010). Additionally, cranial NC cells in Xenopus require expression of cadherin-11 for proper migration (Borchers et al., 2001). Interestingly Snail2 has been also shown to transcriptionally repress cadherin-6B, which belongs to type II classical cadherins in chick cranial and trunk NC cells during delamination (Taneyhill et al., 2007; Coles et al., 2007). The action of Snail2 is supported by interaction with Sox9, another 
transcription factor, which upon phosphorylation, induced by BMP and canonical Wnt signaling, binds directly Snail2 and promotes EMT of chick trunk NC (Cheung and Briscoe, 2003; Liu et al., 2013). Furthermore, canonical Wnt signaling is also thought to be involved in EMT by transcriptional and post-transcriptional regulation of Snail2 (Vallin et al., 2001; Yook et al., 2006). Sox10 and FoxD3 have been also shown to downregulate $\mathrm{N}$-cadherins in the migratory chick trunk NC cells (Cheung et al., 2005; Dottori et al., 2001). What's more, FoxD3 also downregulates cadherin-6B by repressing transmembrane protein tetraspanin18 (Tspan18), which is required for maintenance of cadhrein-6B and prevents delamination of chick cranial NC (Fairchild and Gammill, 2013). FoxD3 additionally up-regulates cadherin-7, which promotes NC motility (Cheung et al., 2005). Further progression of EMT requires dispersion of NC and acquisition of truly mesenchymal fate, what is achieved in later stages of migration. In Xenopus, the dissociation of cranial NC is mediated by Twist, which represses E-cadherin, a type I cadherin, in the delaminating cells. Knockdown of Twist together with its regulator Hifa causes up-regulation of E-cadherin and impairment in NC dispersion (Barriga et al., 2013). In contrast to frog, Twist is not expressed in the avian NC cells during premigratory and migratory stages, however, its role is performed by another transcription factor called Zeb2 (Sip1). In chick, knockdown of Zeb2 leads to maintains of E-cadherin, which normally needs to be downregulated in migratory cranial NC cells. Persistence in expression of E-cadherin does not prevent delamination from the prospective neural tube, but results in the formation of aggregated NC cells remaining in the premigratory position (Rogers et al., 2013). Thus, repression of particular cadherins is crucial for loss of adhesion between NC cells and prospective neural tube, while repression of other cadherins enables NC cells dispersion. 


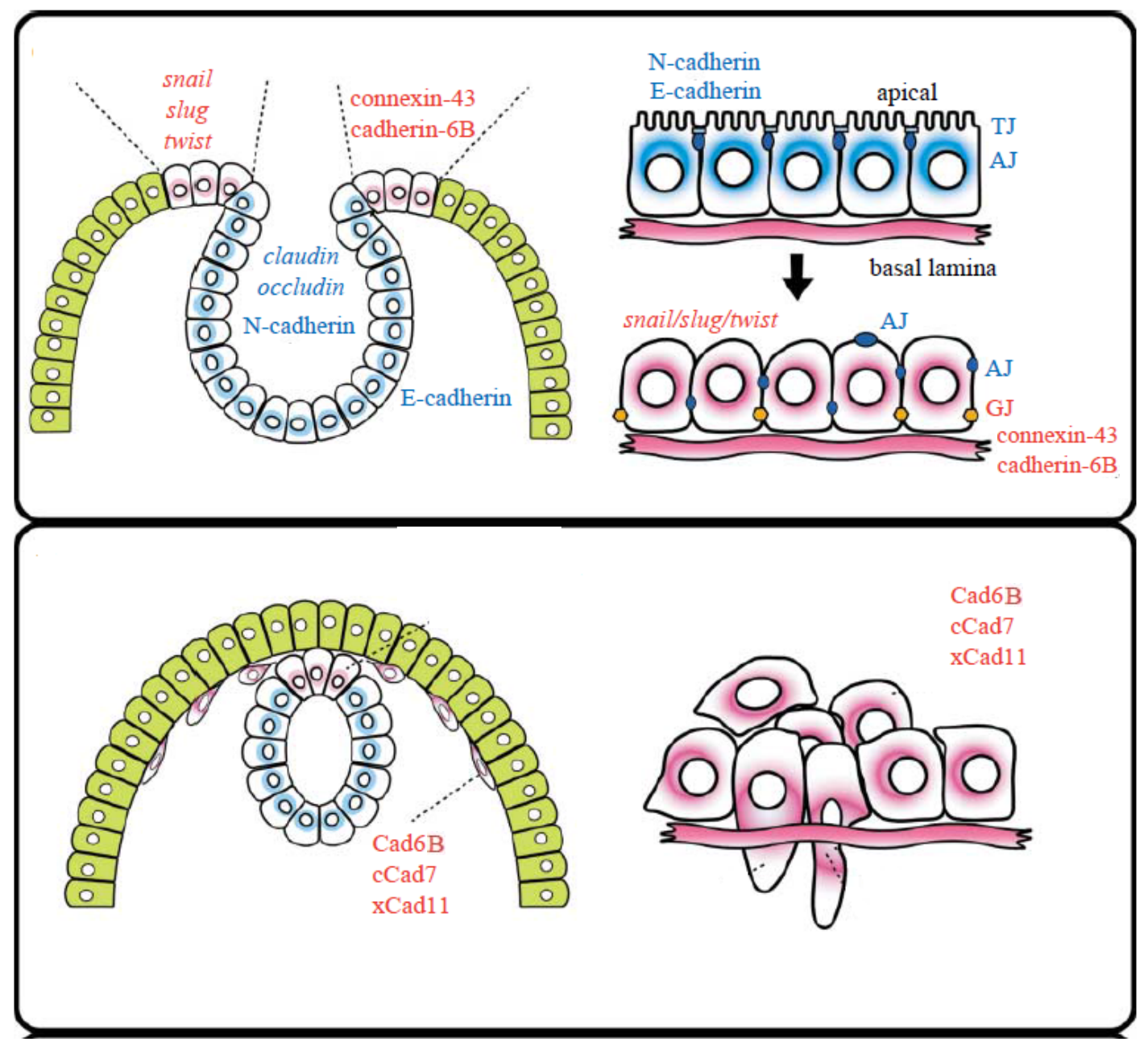

Figure.1.5.1. Loss of epidermal polarity and modulation of cadherin-dependent adhesion. During EMT NC cells lose the epithelial polarity, tight junctions become disassembled and are substituted by gap junctions, what is reflected in the downregulation of occludin and claudins and up-regulation of connexin-43 respectively. Delamination of NC cells from prospective neural tube is additionally facilitated by a switch in expression between class I and II of classical cadherins causing changes in adherens junctions (AJ). Expression of $\mathrm{E}-$ and $\mathrm{N}$ cadherins is usually repressed at the expanse of Cad6B, Cad7 and Cad11. Many NC-specifieres including Snail1/2 and Twist are involved in this processes. Abbreviations: TJ-tight junctions, AJ-adherens junctions, Cad6B- cadherin-6B, cCda7- chick cadherin-7, xCad11- Xenopus cadherin-11. Adapted from Kuriyama and Mayor, 2008.

\subsubsection{Modulation of extracellular matrix}

In addition to the structural rearrangements, delamination of NC cells and active migration throughout the whole embryo involves penetration of the basement membranes and invading extracellular matrices (ECM) (Fig.1.5.2). Migrating NC cells encounter barriers of connective tissue buildup of collagens, fibronectin, laminins, vitronectin and proteoglycans. Proteolytic activity of matrix metalloproteases (MMPs) 
favoring invasive behavior of the metastatic cells is also involved in progression of NC migration, however, in contrast to cancer, NC cells invade surrounding tissue in controlled manner upon regulation by the tissue inhibitors of matrix metalloproteinase (TIMPs) (Fig.1.5.2). Matrix metalloproteinase-2 (MMP-2) and it inhibitor TIMP-2 were shown to be required for the migration of the chick cardiac NC cells. Both MMP-2 and TIMP-2 accumulate on the surface of migrating NC cells and are more abundant at the leading edge in comparison to the trailing edge of the migratory front (Cai et al., 2000; Cantemir et al., 2004; Duong and Erickson, 2004). Also expression of several members of the novel metalloproteinase/disintegrin family ADAM identified in cancer have been reported in Xenopus cranial NC cells (Alfandari et al., 2001; Gaultier et al., 2002; Smith et al., 2002; Harrison et al., 2004). In Xenopus, ADAM13 has been shown to initially decrease adhesion of NC cells to the ECM, facilitate delamination from the prospective neural tube and subsequently enable further migration by cleaving ECM substratum (Fig.1.5.2) (Alfandari et al., 2001; Kee et al., 2007). What's more, ADAM13 has been shown to cleave cadherin-11 generating an extracellular soluble fragment and a membrane-bound cytoplasmic tail, which is crucial for protrusive activities by stimulating small Rho GTPases in cranial NC (Kashef et al., 2009; McCusker et al., 2009). Although the transcriptional control of MMPs/ADAMs proteolytic activity is not fully understood, Zeb2 (Sip1) and Snail2 could be potential regulators of those proteinases (Joseph et al., 2009). After initial degradation of connective barriers further invasion and migration requires additionally formation of contacts between NC cells and proteins of EMC. These interactions are established by integrins (Fig.1.5.2); a large family of transmembrane non-covalently associated $\alpha$ and $B$ subunits, which bind EMC proteins to the cell's actin cytoskeleton. In Xenopus, at least four integrins have been shown to be expressed in favorable temporal and special distribution to function in cranial NC migration (Joos et al., 1995; Kil et al., 1996; Lallier et al., 1996; Ransom et al., 1993; Whittaker and DeSimone, 1993). However, so far only $\alpha 5 ß 1$ was shown to support cranial NC migration on fibronectin (Alfandari et al., 2003). In chick, B1 promotes attachment of trunk NC cells to fibronectin, laminin and collagen (Lallier and Bronner-Fraser, 1991) and more recent studies also confirm involvement of $B 1$ in attachment of cranial NC to fibronectin (Strachan and Condic, 2003, 2008). 

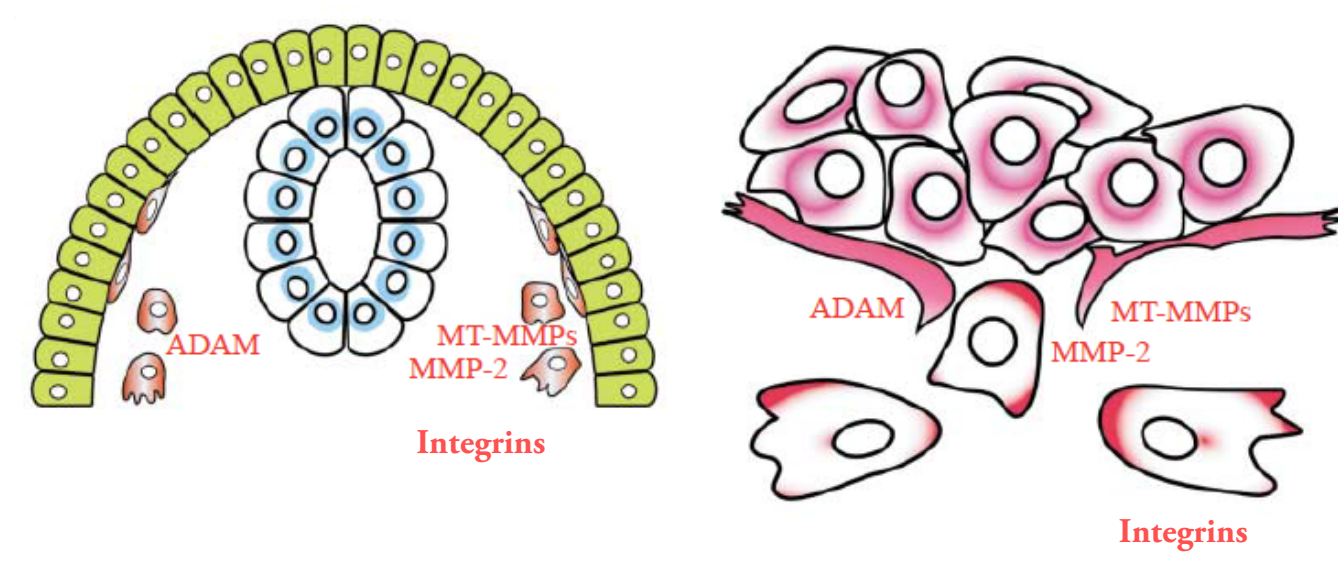

Figure.1.5.2. Interactions with basement membranes and extracellular matrix (ECM). Combined action of MMPS/ADAMs metalloproteinases and integrins facilitates invasion and migration of NC cells through connective tissues. Adapted from Kuriyama and Mayor, 2008.

\subsection{Migration of NC cells}

\subsubsection{Guiding signals-repellents}

After successful completion of delamnination NC cells start active migration throughout the embryo following specific external guiding cues. NC cells migrate as a segmented groups of cell at all axial levels following two stereotypical pathways; a medial pathways leading directly through the anterior-half of the somatic mesoderm or between the neural tube and somatic mesoderm, and a dorsolateral route between the dermamyotome and ectoderm (Fig.1.6.1). During migration NC cells express many genes crucial for migratory behavior, but also initiate a differentiation program, which will lead to formation of various derivatives. After induction, usually during neurulation cranial NC becomes segmented into three groups corresponding to the segmental organization of the brain (Fig.1.6.1) (Sadaghiani and Thiebaud, 1987). Subsequently, those groups of NC cells migrate dorsolateraly into specific branchial arches giving rise to mandibular, hyoid and branchial NC cells (Sadaghiani and Thiebaud, 1987; Bradley et al., 1993; Smith et al., 1997). Only a small amount of cranial NC cells invade the underlying mesoderm (Noden, 1975, 1988). Migration into NC arches is performed in a rostrocadual wave and is temporally separated. NC cells segregated into specific arches contribute to various derivatives like bones, 
cartilages, cranial ganglia and melanocytes. In turn, trunk NC cells migrate in the metameric fashion along two pathways; a medial and dorsolateral route (Fig.1.6.1). Migration via both pathways is also temporally separated. Firstly, NC cells migrating along the medial path generate neurons and glial, of the peripheral nervous system. In a second wave NC cells travel longwise the dorsolateral path and differentiate into melanocytes. In addition, vagal NC cells, regarded as a transition between the cranial and trunk NC cells, were shown to migrate both in a medial and dorsolateral pathway (Kuo and Erickson, 2011). Those NC cells contain a subset of the cardiac NC, which mainly contributes to the connective tissue and smooth muscles (Kirby et al., 1983; Kirby and Waldo, 1995). In all cases, NC cells require a special signaling guidance to assure proper separation of distinct NC cells groups. Interestingly, most of the characterized external guiding cues exhibit a repulsive effect preventing NC cells from entering certain regions and mutual intermingling. Studies in various animal systems proved an essential role of ephrins, semaphorins and slit/robo signaling pathway in directing NC movement (Fig.1.6.1) (Kuriyama and Mayor, 2008).

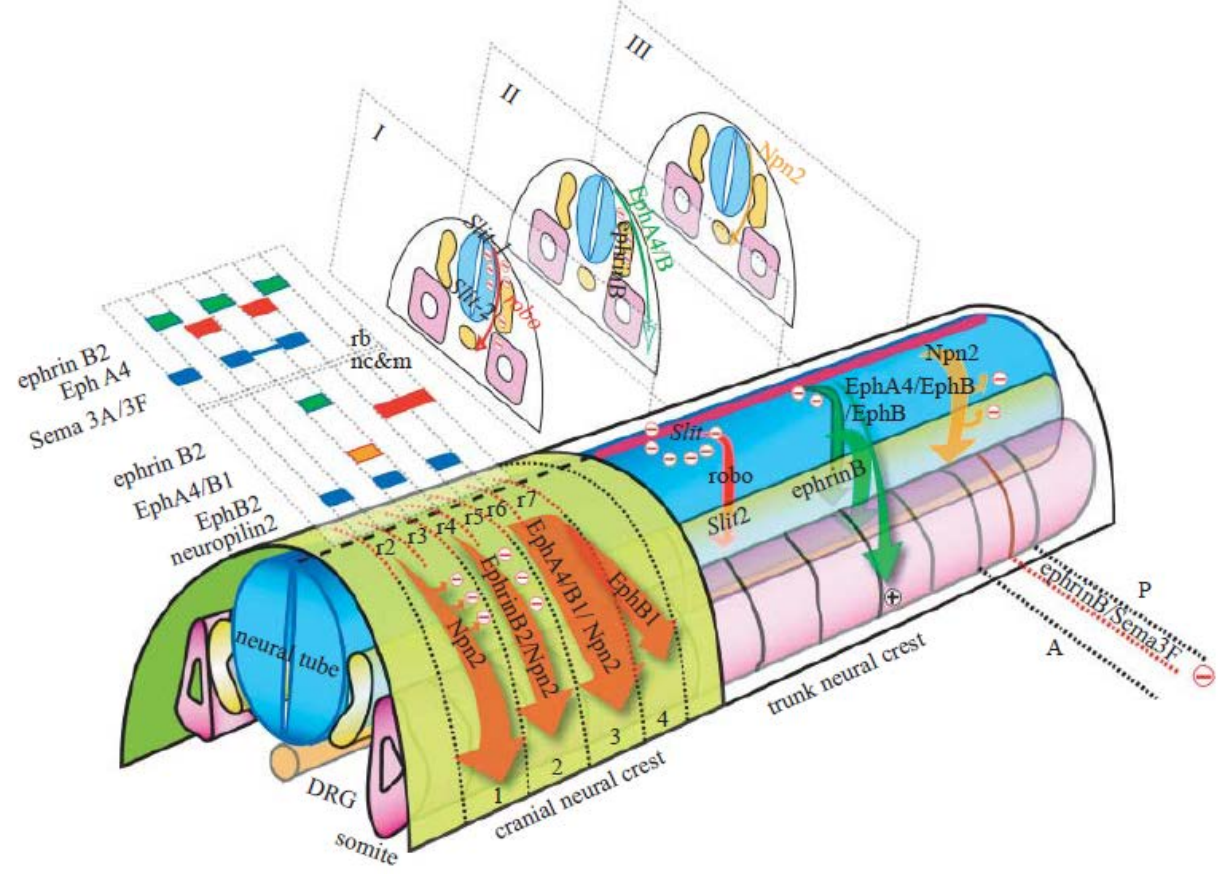

Figure.1.6.1. Repulsive guidance of migrating NC cells. NC cells migrate as segmented group of cells along two stereotypical pathways; medial and dorsolateral. At all axial levels NC cells receive repulsive signals preventing them from spreading and intermingling. Ephirin and semaphoring signaling control migration of cranial NC, while trunk NC are additionally guided by slit/robo signaling. Adapted from Kuriyama and Mayor, 2008. 
Ephrins constitute a family of membrane-bound proteins, which serve as ligands for Eph receptors. Due to their structural differences and varied linkage to the cell membrane they are divided into ephrin-As and ephrin-Bs subclasses. Eprin receptors in turn belong to the superfamily of receptor protein-tyrosine kinases and are classified as either EphAs or EphBs. Although Eph receptors demonstrate high affinity binding for ligands belonging to corresponding classes there are also cases of cross-interactions (Himanen et al., 2004). Activation of the Eph signaling cascade occurs via direct cell-cell interactions, as both ligands and receptors are membranebound proteins. In Xenopus, EphA4 and EphB1 are expressed in migrating cranial $\mathrm{NC}$ cells as well as in mesoderm of the third and fourth arch respectively (Smith et al.,1997; Helbling et al.,1998), while their ligand, namely ephrin-B2 is expressed in migrating NC cells/mesoderm of the adjacent second arch (Robinson et al., 1997; Smith et al., 1997). Using truncated forms of those proteins it has been shown that their interactions provide a repulsive signaling allowing the proper segregation of the second and third-arch NC cells and additionally target third-arch NC cells to their proper destination (Smith et al., 1997). Mutations in human ephrin-B1 and ephrin-B4 are linked with failure of cranial NC migration (Twigg et al., 2004; Merrill et al., 2006), while overexpression of cytoplasmic domain of ephrin-B2 rescues cranial NC migration in ephrin-B2 knockdown mice (Adams et al., 2001). Furthermore, in zebrafish ephrin-B2 has been shown to interact with gap junction protein; connexin43 and regulate its distribution in migratory cranial NC cells (Mellitzer et al., 1999). Avian trunk NC cells invade only anterior, but not the posterior part of each somatic mesoderm due to repulsive guidance cue imposed by the somite. EphB3 expression is localized to the anterior-half of the somite and NC cells, while ephrin-B1 ligand is expressed in the posterior-half of the somite. The addition of soluble ephrin-B1 causes a loss of metameric migration pattern. A similar situation is observed in the murine system where ephrin-B1 and ephrin-B2 ligands are expressed in the posterior-half of the somite, while corresponding Eph receptors are localized exclusively to anterior-half of the somite and truck NC cells (Krull et al., 1997; Wang and Anderson, 1997; Koblar et al., 2000; McLennan and Krull, 2002; KasemeierKulesa et al., 2006; Santiago and Erickson, 2002). Ephrin signaling also controls entering into medial versus dorsolateral pathway by quail trunk NC cells. NC cells which are specified as neurons and glial migrate only via the medial pathway and are prevented from dorsolateral migration into epidermis. On the contrary, NC cells 
specified as melanoblasts are directed into dorsolateral pathway (Santiago and Erickson, 2002). Another group of proteins crucial for proper guidance of NC cells are semaphorins constituting a class of secreted and membrane-bound proteins which were preliminary recognized as axon growth cone guiding molecules (Kolodkin, 1998). Semaphorins can alter the organization of actin filaments and the microtubule network by interaction with neuropilins and plexins receptors, which in turn have well established roles in regulation of Rho-family GTPases (Yu and Kolodkin, 1999). Additionally, recent work shows that Sema4D/plexins-B1 can also influence R-Ras GAP activity and inhibit cell migration by regulating $ß 1$ integrin what could be relevant for NC migration (Oinuma et al., 2006). In zebrafish, Sema3F and Sema3G ligands are expressed in the NC-free cephalic region, while Npl2a and Npl2b are expressed in the migrating NC cells ( $\mathrm{Yu}$ and Moens, 2005). Similarly, in chick Sema3F and Sema3A are expressed in the hindbrain neuroepitelium adjacent to the NC-free mesenchyme, while their neurophilins are expressed in NC cells. Expression of neurophilin-Fc fusion construct causes a migration of NC cells into a NC-free mesenchymal tissue (Osborne et al., 2005; Gammill et al., 2006). Sema3F is also expressed in the posterior-half of the somites where it serves as a repulsive ligand for trunk NC cells expressing Npl2. Sema3F $\%$ and Npl2 $\%$ mutant mice show loss of metameric migration of trunk NC cells. Additionally, in mice Sema3A/Npl1 repulsion prevents population of sacral NC from the premature entrance of the gut (Anderson et al., 2007). In chick, RNAi-mediated knockdown of PlexinA2 or PlexinD1 impairs migration of cardiac NC cells into the outflow tract (Toyofuku et al., 2008). PlexinA1 is expressed in premigratory and migratory cranial NC cells in Xenopus. Both up- and downregulation of PlexinA1 inhibits migration of NC indicating that level of PlexinA1 needs to be finely controlled (Wagner et al., 2010). In addition to ephrin and semaphorin signaling another pathway termed Slit/Robo also plays a role in the NC guidance. Slits are secreted proteins, which bind to the Robo receptors. Slit2 expressed in the chick dermamyotome has been shown to repel early migrating trunk NC cells expressing Robo1 and Robo2 showing that Slit/Robo repulsive interactions are required to prevent the entry of NC cells into the dorsolateral pathway (Jia et al., 2005). Moreover, in quail Slit/Robo interactions prevent migrating trunk NC cell expressing Robo1 and Robo2 from invading gut tissue expressing Slit2 but not the vagal NC cells (De Bellard et al., 2003). 


\subsubsection{Guiding signals-attractants}

Although most of the investigated molecules exhibit repellent interactions restricting NC migration some actually work as attractants. Among them are netrins which belong to family of laminin-related proteins and have been shown to have a dual behavior in axon guidance (Culotti and Merz, 1998; Serafini et al., 1994; Wang et al., 1999). In avian and mice, a subpopulation of vagal NC cells expressing netrin receptors (deleted colon cancer gene (DCC)) migrate toward the developing gut and pancreas expressing netrins. NC cells from explanted intestine were able to migrate out towards the cocultured cells expressing netrin-1, while in vivo blockage of DCC causes loss of directional migration toward target tissues (Jiang et al., 2003). Glial cell line-derived neurotrophic factor (GDNF), belonging to TGFß superfamily also plays a role in attracting a subpopulation of vagal NC expressing receptor tyrosine kinase RET into the prospective gut (Fig.1.6.2.1). Similarly, NC cells from explanted intestine were able to migrate in the direction of GDNF-exogenous source, while RET loss-of-function causes defects in NC migration into target tissue in mice (Natarajan, 2002). Additionally, Endothelin signaling has also been implicated in guiding migratory NC cell, which will contribute to the formation of the enteric nervous system (ENS) (Heanue and Pachnis, 2007). Endothelins are a family of peptides comprising potent vasoconstrictors, which are primaliry expressed by the endothelium (Agapitov and Haynes, 2002). NC cells, which invade the developing gut express Endothelin receptor type $B(E D N R B)$, while Edn3 is expressed in the midgut and hindgut mesoderm as well as in the caecum and proximal colon (Barlow et al., 2003, Leibl et al., 1999) (Fig.1.6.2.1) In mouse, mutations in Edn3 and EDNRB cause delayed migration of gut-destinated NC cells (Barlow et al., 2003; Lee et al., 2003; Ro et al., 2006). Another factor, which has been proposed to attract NC cells is stromal derived factor-1 (Sdf1), a secreted protein which binds transmembrane receptor CXCR4. In mice and chick, expression of CXCR4 is observed in the subpopulation of trunk NC emerging from the neural tube, while Sdf1is expressed along the migration pathway to the dorsal root ganglia (DRG) or sympathetic ganglia respectively. CXCR4 mice show underdeveloped DRG (Belmadani, 2005), while chick NC cells expressing CXCR-shRNA perform slower migration in comparison to control cells. Furthermore, overexpression of CXCR4 in other subpopulations of trunk NC exerts migration to sympathetic ganglia (Kasemeier-Kules et al., 2010). In Xenopus, Sdf1 is expressed in 
the ectoderm facing premigratory and migratory cranial NC cells (Fig.1.6.2.2). Morpholino-mediated knockdown of CXCR4 inhibits NC migration. Interestingly, in both in vitro/in vivo studies NC cells steer their migration in the direction of exogenous/ectopis source of Sdf1 proving its role as an attractant (Theveneau et al., 2010).

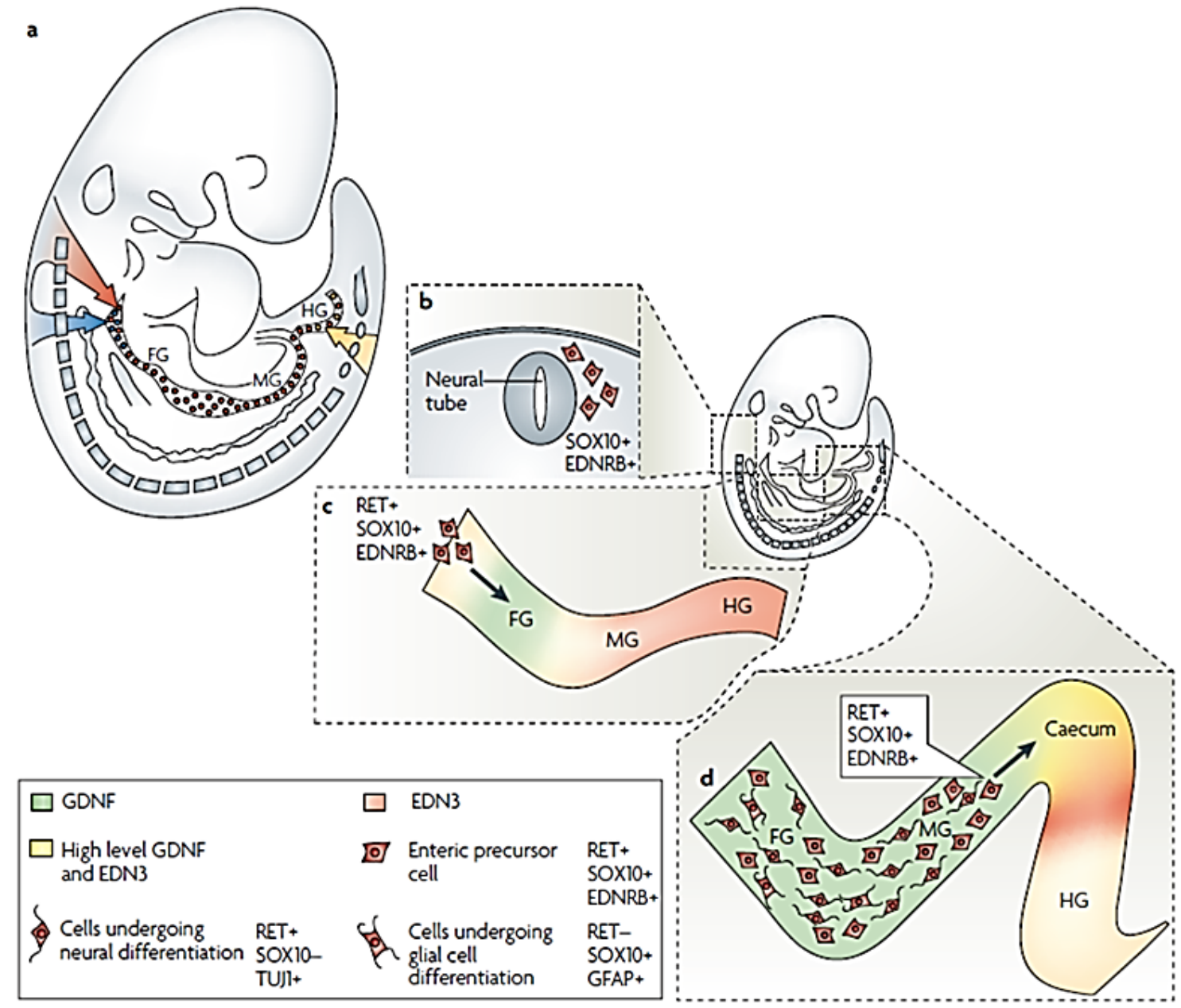

Figure.1.6.2.1. Migration of NC cells contribuits to the formation of the enteric nervous system (ENS). A In the early development of mouse embryos, vagal NC cells (red stream) enter the anterior gut and migrate rostrocadualy to populate foregut (FG), midgut (MG), caecum, and hindgut (HG) respectively. Those NC cells will form the majority of the ENS. The most cadual subpopulation of vagal NC cells together with the most anterior subpopulation of trunk NC cells (blue stream) will contribute to the formation of ENS in oesophagus and the anterior stomach (blue dots). The sacral NC cells positioned at the most posterior part of the developing neural tube (yellow stream) migrate in cadual-rostral direction to colonize the colon (yellow dots). All of the NC cells, which enter the developing gut, are defined as enteric neural crest-derived cells (ENCCS) and will give rise to the ENS. B,C Vagal NC cells migrating anteriorly into the gut express EDNRB and start to express RET, while mesenchyme of the gut expresses high levels of GDNF in foregut (green) and EDN3 in mid- and hingut (pink). D In the later stages, NC cells migrating cadually encounter high levels of GDNF and EDN3 expression in the caecum (yellow). Adapted from Heanue and Pachnis, 2007. 

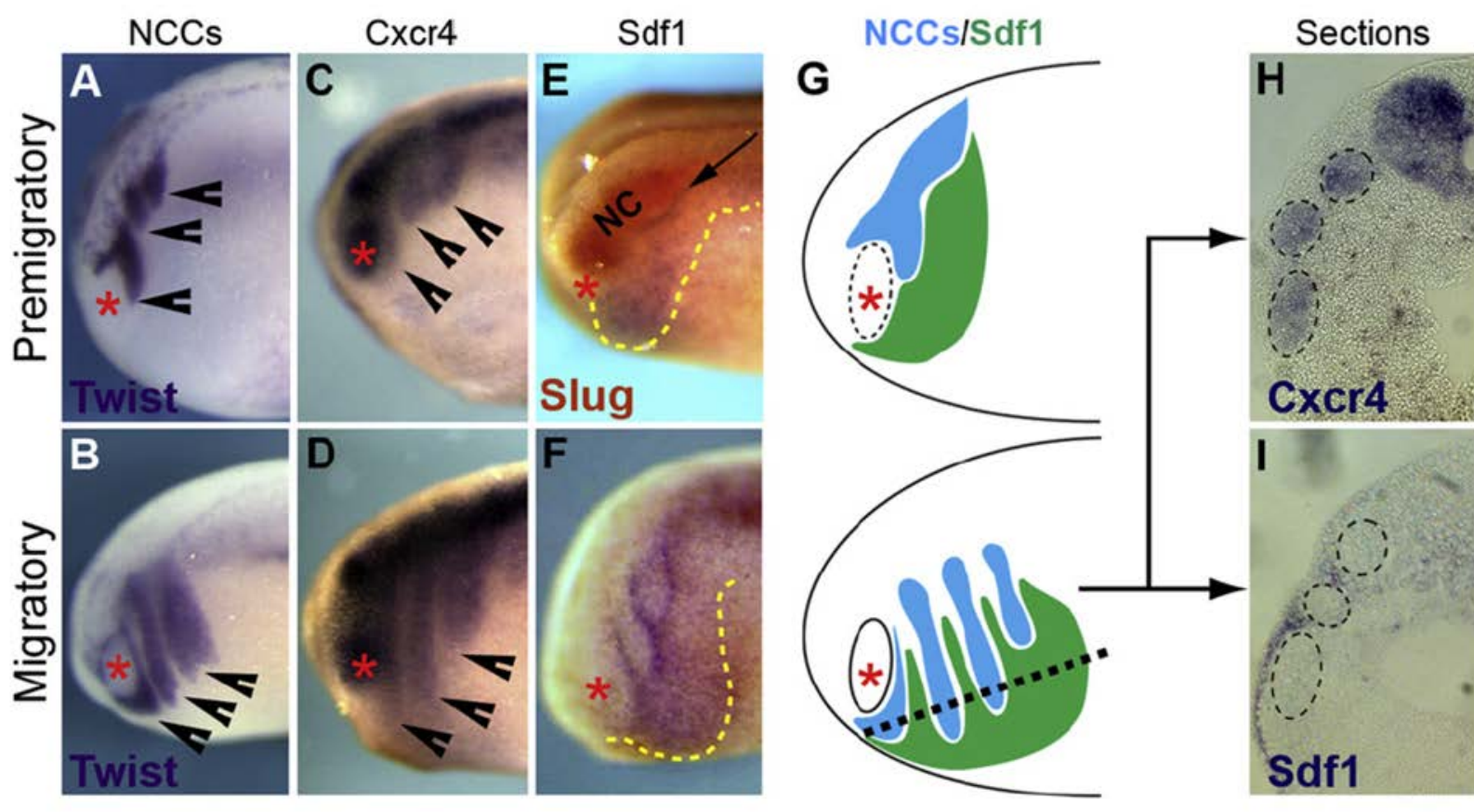

Figure.1.6.2.2. Expression of CXCR4 receptor and Sdf1 ligand. In Xenopus, expression of CXCR4 overlaps with expression of NC-marker genes confirming expression of this receptor by NC cells. Conversely, CXCR4 ligand, namely Sdf1 is expressed in the ectoderm facing premigratory and migratory NC cells. A,B Expression of NC-marker Twist in the premigratory and migratory NC cells. C,D Expression of CXCR4 receptor in premigratory and migratory NC cells. E Expression of Sdf1 ligand facing premigratory NC cells expressing Slug (Snai2) marker. F Expression of Sdf1 ligand in front of the migratory NC cells. G Graphical comparison of premigratory and migratory NC cells and expression of Sdf1 ligand. $\mathbf{H}$ Horizontal sections of Xenopus embryos showing expression of CXCR receptor and Sdf1 ligand. Adapted from Theveneau et al., 2010.

\subsubsection{Establishment of cell polarity}

Once NC cells undergo delamination, they migrate with very persistent polarity either as clusters or as individual cells in the direction of a target tissue. A prerequisite for directional migration is a formation of front-back polarity. In vitro analysis of singlecell migration revealed that cell protrusions arise at the front and retract at the back of cells respectively through the localized assembly of the actin controlled by the Rho family of small GTPases (Fig.1.6.3). At the very tip of the cell front cdc42 GTPase controls actin polymerization, which becomes organized in the parallel bundles and contributes to formation of filopodia and nascent cell-substratum adhesion (Itoh et al., 2002). More backwards, but still at the front Rac1 GTPase regulates actin polymerization, which forms a mesh-like network controlling lamellipodia extension and formation of nascent cell-substratum adhesion. Rac1 directly activate the WAVE family of actin nucleators and phosphoinositides, which are required for activity of 
actin-nucleating proteins including N-WASP-Arp2/3 complex (Burridge and Wennerberg, 2004; Ridley et al., 1992; Rohatgi et al., 2001). Finally, at the rear RhoA small GTPase promotes assembly of stress fibers and mature focal adhesions (Burridge and Wennerberg, 2004; Ridley and Hall, 1992). RhoA activates Rho kinase (ROCK), which in turn activates PTEN and causes actomyosin-based contractility, protrusions collapse and finally cell translocation (Chrzanowska-Wodnicka and Burridge, 1996; Li et al., 2005; Ridley et al., 2011). RhoA and Rac1 have also been shown to mutually antagonized each other at the front and back (Bustos et al., 2008; Ohta et al., 2006)

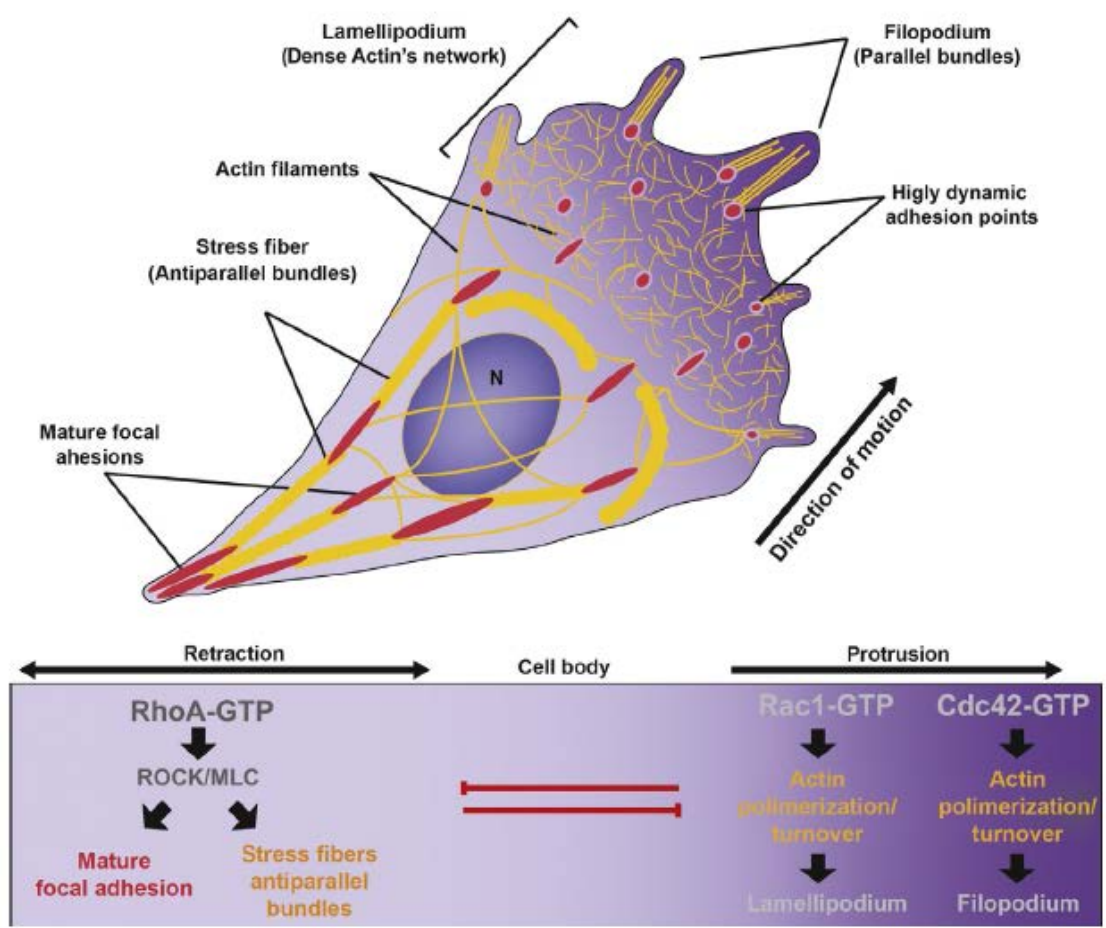

Figure.1.6.3. Small GTPases and acting remodeling. Polarized activities of Cdc42, Rac1 and RhoA are responsible for the establishment of front-back polarity of the migratory cell. At the front Cdc2 and Rac1 promote protrusion activity, while at the back Rho controls protrusion disassembly. Adapted from Barriga and Mayor, 2015.

\subsubsection{Mechanism of planar cell polarity (PCP)}

Activation of proteins belonging to the Rho family of small GTPases occurs mainly through the planar cell polarity (PCP) pathway, which was extensively studied in the fruit fly Drosophila melanogaster where it controls the proximal-distal orientation of hair and bristles on the body (Klein and Mlodzik, 2005; Tree et al., 2002; Adler 2002). Genetic screening of Drosophila mutants revealed a set of 'core PCP' proteins, which drive localized actin polymerization responsible for the localized growth of hair and 
bristles. The 'PCP' core components include several transmembrane proteins, such as Frizzled (Fz) (Adler et al., 1997; Vinson and Adler, 1987), Strabismus (Stbm)/Van Gogh (Vang) (Taylor et al., 1998; Wolff and Rubin, 1998), Flamingo (Fmi) (Chae et al., 1999; Usui et al., 1999) and intracellular proteins like Disheveled (Dsh) (Theisen et al., 1994), Prickle (Pk) (Gubb et al.,1999) and Diego (Dgo) (Feiguin et al., 2001). In the Drosophila wing cells, all of those proteins are initially uniformly distributed in the whole cell membrane, however, upon mutual interactions they become asymmetrically segregated; Fz, Dsh and Dgo accumulate at the distal and Stbm/Vang and Pk at the proximal edge of the cell membrane (Axelrod, 2001; Strutt, 2001; Feiguin et al., 2001; Tree et al., 2002; Bastock et al., 2003). The atypical cadherin, Fmi resides on both distal and proximal edges, where it binds neighboring cells through heomophilic interactions (Usui et al., 1999; Shimada et al., 2001). Establishment of PCP is the best understood in Drospohila and involves intra and intercellular interactions. The initial asymmetrical proximal-distal distribution of FzFmi and Stbm/Vang-Fmi is most likely caused by preferential recruitment of Fz to the cell membrane by Fmi by so far unknown mechanism (Chen et al., 2008; Strutt and Strutt, 2008; Struhl et al., 2012). Fz-Fmi complexes interact with Stbm/Vang-Fmi complexes, which are localized in the membranes of the adjacent cells, however, the same complexes, anchored in the proximal and distal edges of the same cell antagonizes each other. This repulsive interaction between Fz- and Stbm/Vangcomplexes is performed by cytoplasmic proteins; Dgo and Pk, which can bind the same domain of Dsh (Fig.1.6.4). Overexpression of Pk was shown to prevent Dsh translocation to the membrane, suggesting that $\mathrm{Pk}$ can displace Dsh from the proximal edge of the cell (Tree et al., 2002; Carreira-Barbosa et al., 2003). On the contrary, Dgo binding to Dsh at the distal edge of the cell would prevent association with $\mathrm{Pk}$ and promote distal localization of Dsh. In vertebrates embryos, such as Xenopus, zebrafish and the mouse, the same set of 'core PCP' proteins has been shown to regulate a plethora of early developmental events ranging from convergent extension movements during gastrulation, orientation of cochlear hair cell in the ear, neural tube closure, oriented cell division, lung branching, hair follicle alignment and polarized ciliary beating in the trachea and brain ventricles (Goodrich and Strutt, 2011, Gray et al., 2011; Zallen, 2007; Bosoi et al., 2011; De Marco et al., 2012; Kibar et al., 2009; Kibar et al., 2007; Rida and Chen, 2009; Wang and Nathans, 2007; Yates and Dean, 2007; Segalen and Bellaïche, 2009; Vladar et al., 2012) 
(Fig.1.6.4.1). In all cases local modulation of cytoskeleton is crucial for the proper conduct of these processes.
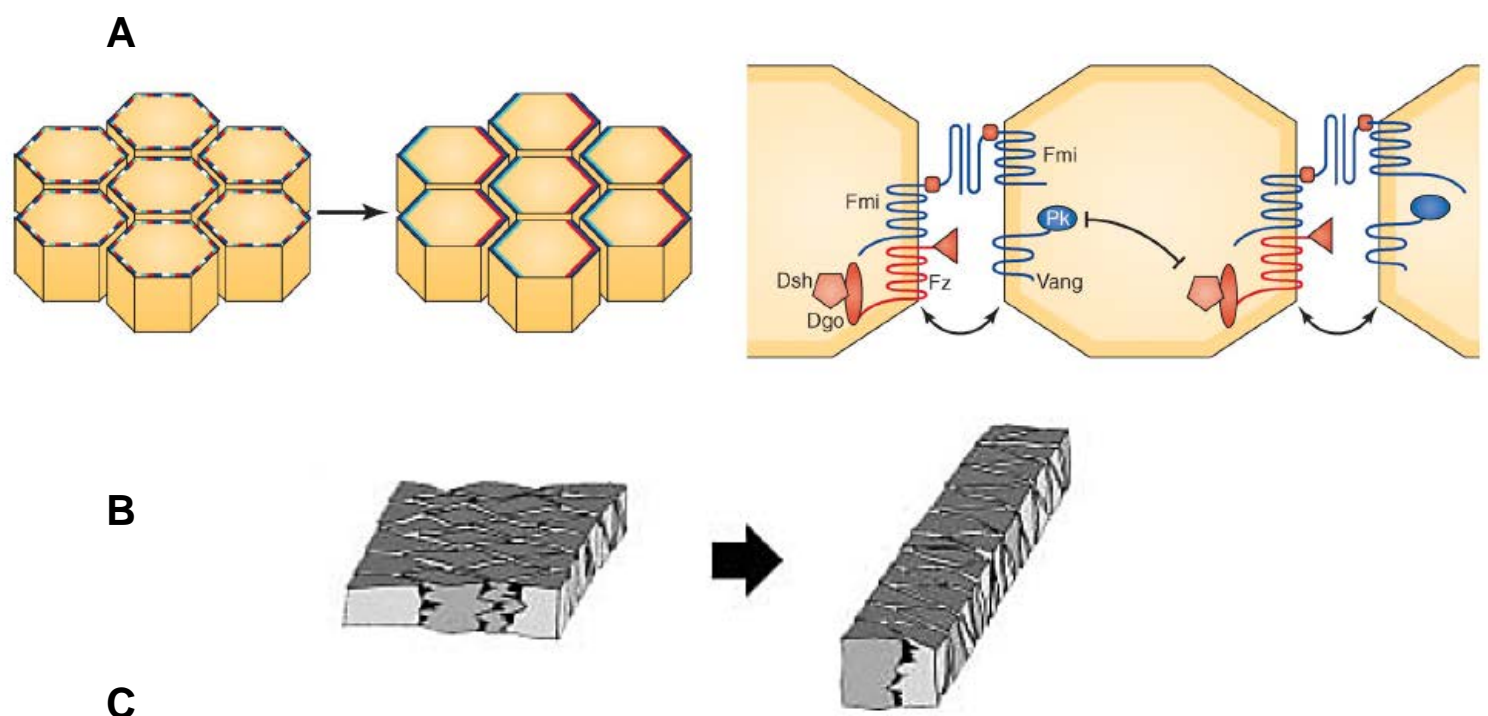

C

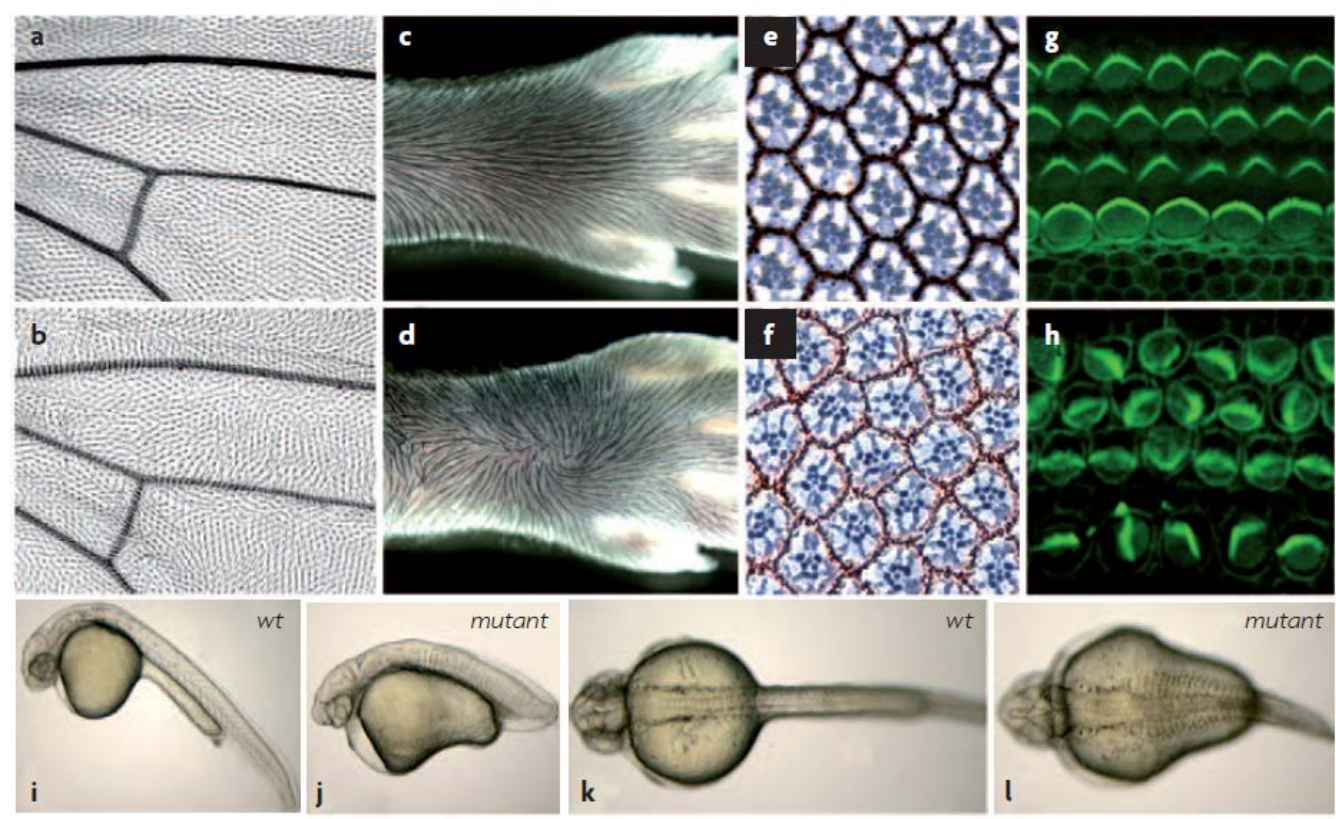

Figure.1.6.4.1. Planar cell polarity in invertebrates and vertebrates model organisms. A In the wing of Drosophila PCP is established by the asymmetrical distribution of 'core PCP' components. Fz, Dsh and Dgo are localized to distal/posterior end, while Vang and Pk are localized to proximal/anterior end. Fz-Dsh-Dgo and VangPk interact extracellulary, but antagonize each other intracellulary. B PCP controls convergent extension (CE) movements which narrow the later tissues and elongate the embryo along anterior-posterior axis. $\mathbf{C}$ Defects in PCP caus; misorientation of wing hair cells in the fly (b), misorientation of mice hair cells (d), misorientation of rhabdomers in ommatidia (f), misorientaion of inner ear hair cells (h), shortened and widened body axis due to impaired CE. Adapted from Deventpor, 2014, Seifert and Mlodzik, 2007.

Similarly to the canonical Wnt pathway, Wnt ligands are also involved in PCP. In vertebrates, Wnt ligands which do not cause stabilization of $\beta$-catenin such as Wnt5a, Wnt5b, Wnt9b and Wnt11 were shown to activate PCP signaling in various 
processes. What's more, those ligands also act as a global directional cues, which orient PCP in a group of cells. In zebrafish, mutations in Wnt5b (pipetail) and Wnt11(silberblick) interfere with convergent extension movements. Elongation of the body axis is impaired and phenocopies mutation in core PCP protein Vangl2 (Heisenberg et al., 2000; Jessen et al., 2002; Rauch et al., 1997). Orientation of sensory hair cells in inner ear and neural tube closure in mice is regulated by Wnt5a and also involves Vangl2 (Qian et al., 2007). Additionally, Wnt5a is required for establishing PCP in the developing murine limb (Fig.1.6.4.2). Gradient of Wnt5a along the developing limb bud causes a gradient in Vangl2 phosphorylation which is translated into asymmetrical localization of this protein in chondrocytes and polarized cell behavior. Gradients of Wnt5a were also described in the facial primordia, tail bud and tongue where most likely are responsible for regulating PCP (Fig.1.6.4.2) (Gao et al., 2011). In chick, expression of Wnt11 in the neural tube controls the alignment of skeletal muscle fibers along the body axis by providing the directional cue for PCP (Gros et al., 2009). Convergent extension and polarized divisions of the kidney epithelial cells, which maintain proper diameter of kidney tubules, are controlled by Wnt9b through PCP. In mice, mutations in Wnt9b disturb PCP causing an increase in diameter of kidney tubules which contributes to cystogenesis (Karner, 2009).
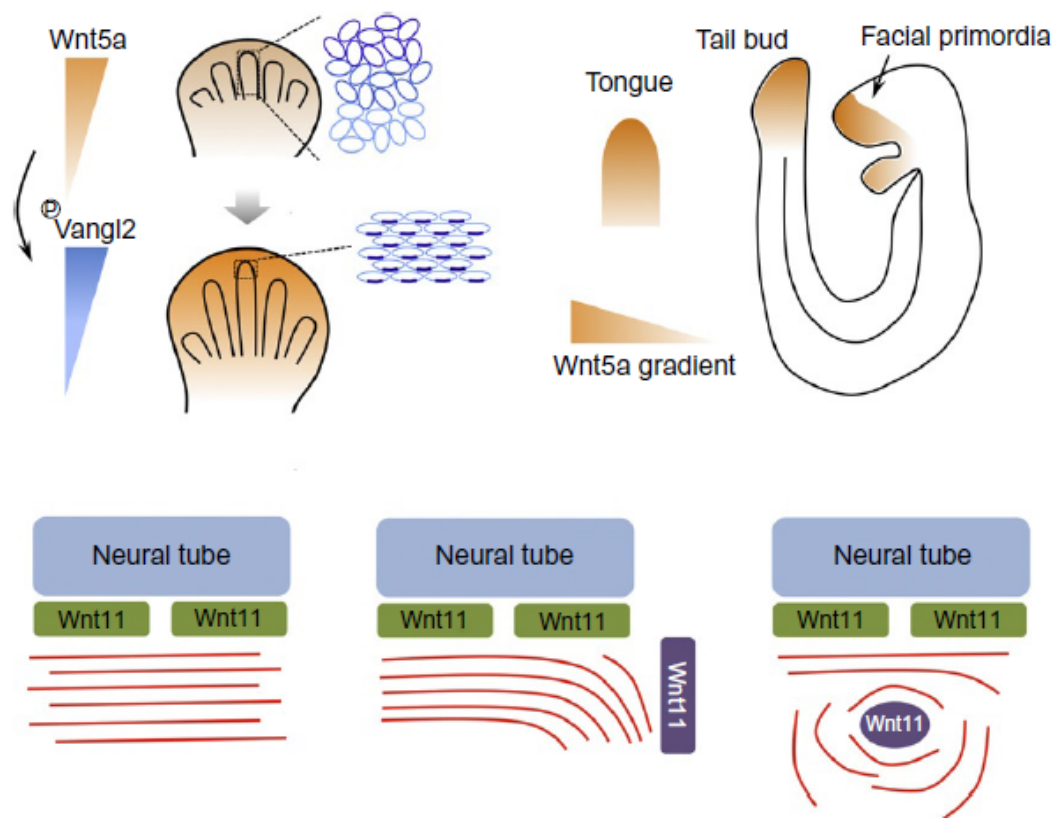

Figure.1.6.4.2. Wnt ligands as a source of PCP directional cues. In mouse embryo, gradient of Wnt5a establishes a gradient of Vang activity, which results in a polarized character of cells building up limbs and most probably tongue, tail bud and facial primordial. In the chick embryo, Wnt11 provides a PCP directional cue for the muscle cells aligned along the neural tube. Ectopic source of Wnt11 causes alignment of muscle cells along the source. Adapted from Gao, 2012. 
Binding of non-canonical Wnt ligands to the Frizzled receptor includes recruitment of Dsh to the cell membrane, but on the contrary to the canonical Wnt pathway, interactions with downstream targets is performed by the PDZ and DEP domains (Cheyette et al., 2002; Wong et al., 2003; Heisenberg et al., 2000; Moriguchi et al., 1999; Tada and Smith, 2000; Wallingford et al., 2000). Once Dsh is properly localized it affects cytoskeleton rearrangement via two distinct small GTPases-dependent pathways, namely Rho- and Rac- pathways. In the Rho-pathway, Dsh associates with Disheveled-associated activator of morphogenesis 1 (DAAM1), which resides in the cytoplasm at the autoinhibited state achieved by intramolecular binding between $\mathrm{N}$ - and C-terminal domains (Fig.1.6.4.3A) (Alberts, 2002; Higgs, 2005). Dsh activates DAAM1 through interaction with its C-terminal domain (Liu et al., 2008). Subsequently, Dsh as well as activated DAAM1 activate Rho through a guanine exchange factor WEGF (weak-similarity GEF), which in turn activates Rhoassociated kinase (ROCK) and remodels actin cytoskeleton. WEGF has been reported in regulation of convergent extension during Xenopus gastrulation (Habas et al., 2001). Additionally, DAAM1 can also mediate actin modifications by interaction with the actin-binding protein Profilin1, which has been shown to regulate blastopore closure during gastrulation of Xenopus embryos (Fig.1.6.4.3A) (Matusek et al., 2008; Sato et al., 2006). In the Rac-pathway, Dsh activates Rac, which then activates cJun N-terminal kinase also involved in actin modulation (Fig.1.6.4.3A) (Habas et al., 2001). The activation of cdc- 42 , which is required for the formation of filipodia usually occurs through the $\mathrm{Wnt} / \mathrm{Ca}^{2}+$ pathway, however, it was also shown to be a downstream transducer of 'core PCP' proteins (Schlessinger et al., 2007; Tada and Kai 2009). Transduction of the signal through the PCP pathway also requires binding of co-receptors to Wnt/Fz complexes, but unlike canonical Wnt pathway Lrp5/6 is not involved. Instead PTK7, ROR2 or Ryk, have been reported to work as co-receptors in PCP signaling (Fig.1.6.4.3B). 
A

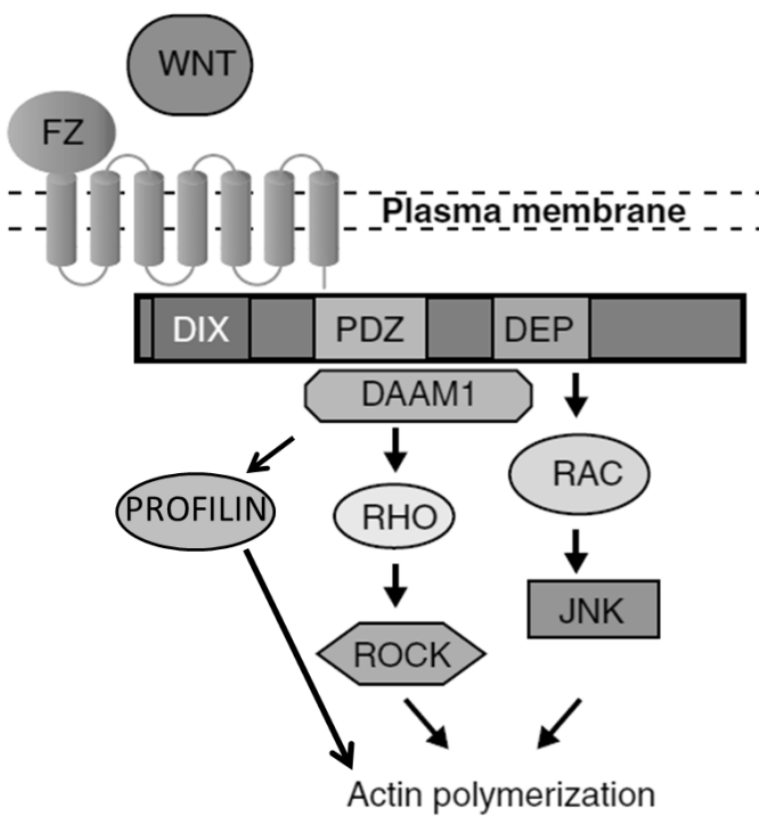

B

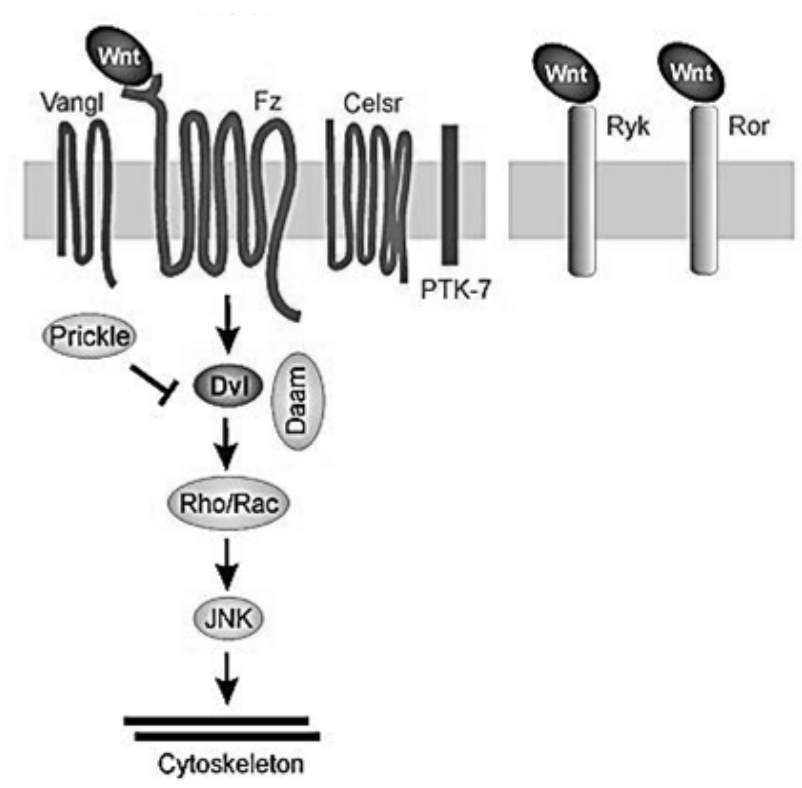

Figure.1.6.4.3. A Two branches of the PCP pathway; Dsh-Rac1 and Dsh-DAAM1. Actin modification and thus polarized cell behavior can be achieved by direct interaction of Dsh with Rac1 or interactions with DAAM1, which in turn interacts with Rho or Profilin causing localized actin assembly. B Atypical cooreceptores regulating PCP pathway. Several single transmembrane proteins classified as tyrosine kinase receptors with dead-kinase domain were shown to participate in regulation of PCP signaling. Among them are; PTK7, Ryk and Ror2. Adapted from Wallingford and Habas, 2005; Avilés et al., 2013.

Protein tyrosine kinase 7 (PTK7) with defective kinase domain is a single transmembrane protein belonging to the family of receptor tyrosine kinases. Also known as colon carcinoma kinase 4 (CCK4), PTK7 was initially identified in colon cancer where its up-regulation is linked with high metastatic. In mice, PTK7 has been shown to interact genetically with Vangl2 in regulating neural tube closure and polarity of inner ear hair cells (Lu et al., 2004). In addition, PTK7 was reported to regulate convergent extension movements during gastrulation both in mice and Xenopus (Yen et al., 2009; Lu et al., 2004).

Receptor tyrosine kinase like orphan receptor 2 (Ror2) is a single transmembrane protein belonging to receptor tyrosine kinase like orphan receptor (Ror) family. Ror2 interaction with Wnt5a is well established and is classified as a separated branch of the Wnt pathway, namely Wnt5a/Ror2 pathway (Green et al., 2008; Minami et al., 2010). In Xenopus, activation of Wnt5a/Ror2 pathway was shown to promote 
expression of paraxial protocedherin (XPAPC), which promotes convergent extension movements by acting through PI3K, Cdc-42, MKK7, JNK and c-jun (Schambony and Wedlich, 2007). In mice, double knockdowns of Wnt5a and Ror2 cause a plethora of anatomical defects, which phenocopy defects observed in individual with Robinow syndrome caused as well by mutations in Wnt5a and Ror2 (DeChiara et al., 2000; Gao et al., 2011; Oishi et al., 2003; Takeuchi et al. 2000; Afazal et al., 2000). Vangl2 $\%$ mutants mice have similar defects as Wnt5a $\%$ and Ror2 $\%$. Furthermore, a biochemical study demonstrated that Wnt5a induces complex formation between Ror2 and Vangl2 indicating that Ror2 is involved in regulation of PCP signaling (Gao et al., 2011).

Receptor-like tyrosine kinase Ryk is another single transmembrane receptor with defective kinase domain involved in regulating PCP. In mice, Ryk $\%$ mutants show defects in the orientation of sensory hair cells in the inner ear and genetic interactions with Vangl2 \% mutants (Macheda et al., 2012). Additionally, Ryk was also found to genetically interact with Wnt11 during convergent extension movements in zebrafish (Kim et al., 2008).

\subsubsection{Role of PCP in NC migration}

Several studies across various vertebrates' models confirmed the requirement of the PCP pathway in NC migration. Expression of Wnt11 and Wnt11R ligands was observed in the ectoderm next to premigratory NC cells just before the delamination in Xenopus (De Calisto et al., 2005; Garriock and Krieg, 2006). What's more, inhibition of Wnt11 through overexpression of dominant-negative construct leads to a complete inhibition of NC migration (De Calisto et al., 2005). In zebrafish, mutations of Stbm/trilobite (Jessen et al., 2002), Wnt11/silberblick (Heisenberg et al., 2000) or Wnt5/pipetail also causes a defect in directional NC migration (Rauch et al., 1997; Matthews et al., 2008). Injections of dominant-negative Dishevelled inhibiting PCP signaling but not canonical Wnt signaling inhibit migration of NC cells in Xenopus (De Calisto et al., 2005) and severely disrupt the directional migration of NC cells in zebrafish (Matthews et al., 2008). In Xenopus and mice, expression of 'core PCP' components including Stbm/Vang, Prickle and DAAM was observed in the cranial NC (Bekman and Henrique, 2002; Darken et al., 2002; Goto and Keller, 2002; Nakaya et al., 2004). Depletion of the syndecan-4, a transmembrane modulator of PCP 
signaling, which co-operated with integrins during cell-matrix interactions, reduces the directional migration of NC cells in Xenopus. Loss of syndecan-4 causes uncontrolled formation of lamellipodia in all directions as an outcome of upregulated Rac activity (Fig.1.6.5). Additionally, depletion of Dishevelled causes the same effect on migration/protrusion orientation through downregulation of RhoA activity which elevates Rac activity (Fig.1.6.5) (Carmona-Fontaine et al., 2008). FRET analysis of $\mathrm{NC}$ cell migrating in vivo revealed that interaction of syndecan-4 with fibronectin is responsible for spatial modulation of Rac and Rho activity. Delaminated NC cells come in contact with fibronectin, which constitutes the main substrate for NC migration. Interactions of fibronectin with syndecan-4 causes the gradient of Rac activity across the cell with the highest enrichment at the leading edge where it promotes formation of lamellipodia required for directional migration. PCP signaling becomes localized to the back of the cell where it promotes Rho activity and simultaneously inhibits Rac thereby preventing formation of protrusions (CarmonaFontaine et al., 2008).

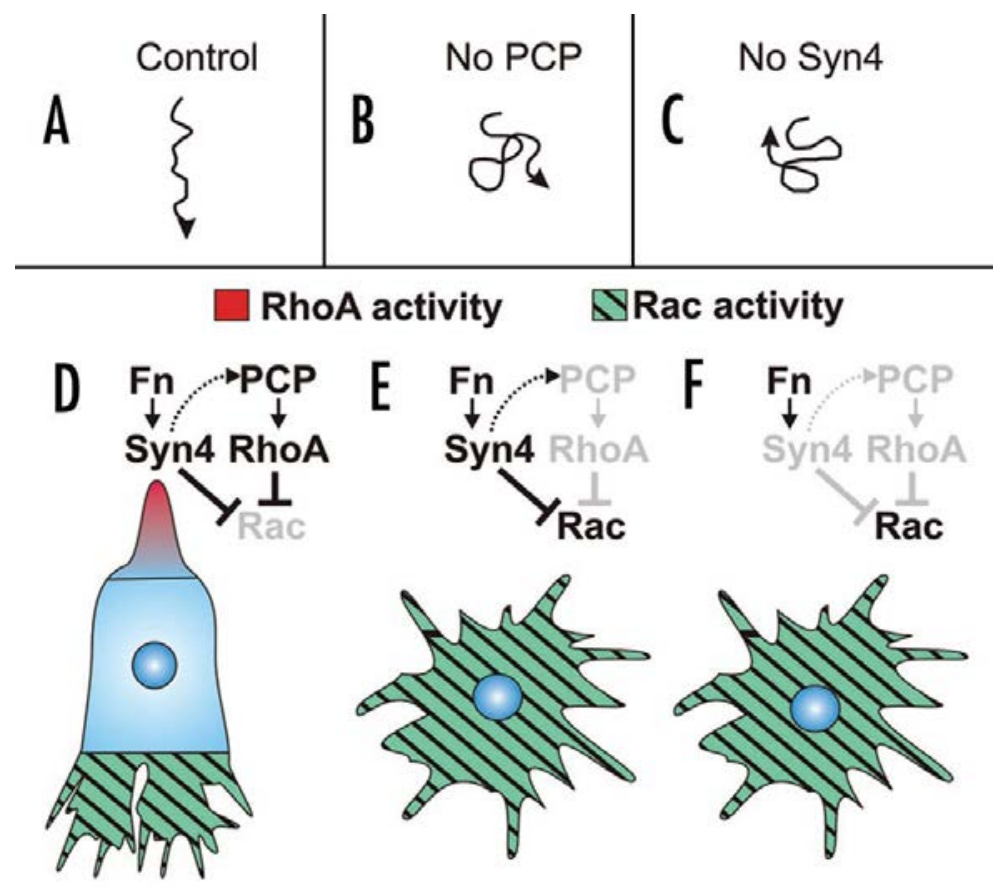

Figure.1.6.5 A,B,C Exemplary tracks of control NC cell and NC cells with impaired PCP or Syn4 signaling. Lack of PCP and Syn4 signaling causes loss of directional migration. D Activation of PCP and Syn4 signaling results in inhibition of Rac activity at the back of the cell leading to cell polarization and directional migration. E,F Inhibition of PCP and Syn4 increases Rac activity at the back of the cell leading to ubiquitous formation of cell protrusions. Adapted from Carmona et al., 2008. 
In Xenopus, PTK7 which has been shown to modulate PCP signaling in many developmental processes is expressed in premigratory and migratory cranial NC cells. Morpholino-mediated knockdown of PTK7 causes complete inhibition of NC migration. What's more, in vitro studies revealed that PTK7 recruits Dsh to the plasma membrane and this interaction depends on Dsh-PZD domain and defective kinase domain of PTK7. Immunoprecipitation assay demonstrated that Frizzled-7 is required for PTK7-mediated recruitment of Dsh. In vivo, NC-specific overexpression of PTK7 construct lacking kinase homology domain also leads to a dramatic inhibition of migration. Interestingly, NC-specific overexpression of Dsh- $\triangle \mathrm{PZD}$ causes only mild migration defects in comparison to defects observed upon overexpression of Dsh$\triangle D E P$ what indicates that PTK7 mediates PCP signaling in NC (Shnitsar and Borchers, 2008). Taken together these data confirm a requirement of PCP signaling in the migration of NC cells.

\subsubsection{Cell-cell interactions in establishment of PCP}

During migration NC exhibit typical mesenchymal morphology and maintain cadherindepended interactions, which are also crucial in performing directional movement by abolishing cell protrusion at contact points and restricting protrusive activity to the front. Studies in epithelial as well as non-epithelial cells revealed several steps in establishment of cadherin-dependent cell-cell contacts. Initially, exploring cells use large protrusions to scan the surrounding for potential binding partners. Establishing a cell-cell contact results in accumulation of cadherins at the contact point, which bind adjacent cells together through calcium-dependent heomophilic interactions (Cavey and Lecuit, 2009; Meng and Takeichi, 2009). During the initial step of exploration, at the very first moment of contact cells exhibit a transient peak of Rac1 activity allowing for further exploration, which is immediately inhibited by activation of RhoA upon formation of adherents junctions (Perez et al., 2008). Activation of RhoA also leads to the disassembly of actin filaments and retraction of lamellipodia (Baum and Georgiou, 2011; Vasioukhin et al., 2000; Yonemura et al., 2011). Initial contact sites are regarded as footholds for lateral expansion of adherent junctions, which occurs outward in alternated rounds of activation and inhibition of Rac1 and RhoA activities. Subsequently myosin-mediated tension pulling inward on the contacts sites 
mechanically facilitates expansion of adherent junctions (Cavey and Lecuit, 2009). And finally, maintenance and stabilization of adherence junctions is achieved by downregulation of actin turnover at the contact points (Pilot et al., 2006; Zandy et al., 2007). In various epithelial and mesenchymal cells which perform migration, similar process contributes to contact inhibition of locomotion (CIL), a cellular behavior causing retraction of cell protrusions upon cell-cell contact. In migratory group of cells, CIL prevents formation of protrusions between neighboring cells. Filopodia and lamellipodia are only formed at the leading edge imposing directionally for the migrating collective. In singularly migrating cells, which collide with each other, CIL enable collapse of protrusion at the contact point, repolarization and migration in the opposite direction (Shih and Yamada, 2012; Carmona-Fontaine et al., 2008; Heckman, 2009). In Xenopus and zebrafish, N-cadherin has been shown to cause local activation of PCP signaling in colliding cranial NC cells. Activation of RhoA results in inhibition of Rac1 activity at the contact points and establishment of cell protrusion at the opposite ends (Carmona-Fontaine et al., 2008; Theveneau et al., 2010; Theveneau and Mayor, 2011b). Recently, cadherin-11 was shown to promote $\mathrm{CIL}$ in colliding NC cells by mediating cell-cell adhesion, however, mechanism orchestrating this process requires further investigation (Becker et al., 2013). Taking in consideration that $\mathrm{CIL}$ causes repolarization of cells after contact, one could assume that it would lead to complete dispersion of NC cells and loss of collective migration. Interestingly, NC cells studied in vitro, in the absence of repulsive signaling, which would restrict their migration in vivo, are still able to migrate as a group. Recently, it has been shown that NC cells are able to mutually attract each other. In Xenopus, migrating cranial NC cells express the complement fragment C3a and cognate receptor $\mathrm{C} 3 \mathrm{aR}$ both well characterized in chemotaxis in immune system (Carmona-Fontaine et al., 2008; Ricklin et al., 2010). Concentration of C3a is higher in area with higher density of NC what allows C3aR-positive cells, which detached from the group after CIL to be attracted back to the cluster (Fig.1.6.6). 


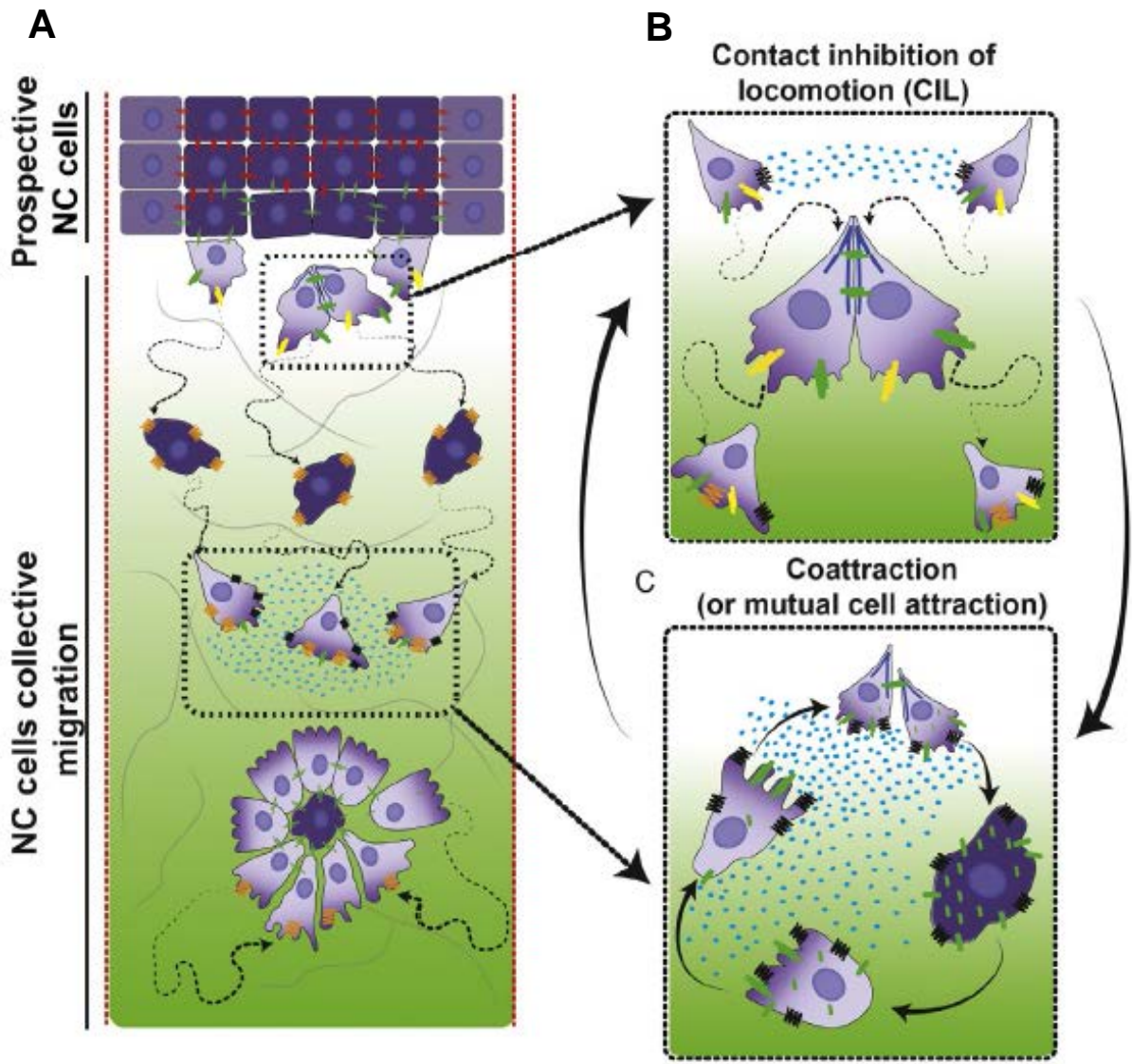

Key:

\begin{tabular}{|c|c|c|c|c|c|}
\hline \multicolumn{2}{|c|}{ Chemotaxis: } & \multicolumn{2}{|c|}{ Adhesion molecules: } & \multicolumn{2}{|c|}{ Coatracttion: } \\
\hline w & Cxcr4 & $=$ & Type-II cadherin & 0 & C3a \\
\hline & Low $\rightarrow>$ high Sdf1 gradient & $=$ & $\mathrm{N}$-cadherin & $m$ & C3aReceptor \\
\hline CIL: & & $=$ & E-cadherin & & \\
\hline & RhoA $\rightarrow$ Rac1 activity gradient & 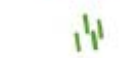 & $\mathrm{N}$-cadherin fragments & & \\
\hline & Wnt/PCP signal & $\cdots$ & Restrictive signals & & \\
\hline
\end{tabular}

Figure.1.6.6. Cross-talk of chemotaxis, contact inhibition of locomotion and coattraction during collective migration on NC cells. A Repulsive signaling is represented as parallel dotted lines. Concentration of Sdf1 is represented as green gradient. After delamination from prospective neural tube, NC experience CIL what results in cell repolarization and dispersion. Single NC cells become polarized in the direction of increasing Sdf1 concentration. NC cells following Sdf1 gradient encounter each other and form transient cell-cell contacts. Upon new contacts NC cells again undergo repolarization and dispersion. Alternating cycles of CIL and Sdf1 coattraction are proposed to impose directive migration of transient NC clusters. B Colliding NC cells form transient $\mathrm{N}$-cadherin-dependent contacts, which promote activation of PCP signaling. Repression of Rac1 and activation of RhoA at the contact side leads to cell repolarization and migration in opposite directions. C Additionally, repolarized NC cells, which experienced CIL can mutually coattract each other through expression of C3a and C3aR. NC cells form transient collectives and again undergo CIL. Adapted from Barriga and Mayor, 2015. 


\subsection{Cross-talk of Wnt pathways}

The classical view of Wnt signaling assumes participation of particular components either in canonical or non-canonical Wnt pathway. Currently accumulating evidence shows that Wnt ligands can transduce signals through both pathways depending on the tissue-specific combination of receptors (van Amerongen et al., 2008, Mikels and Nusse, 2008). What's more, several studies indicate that activation of one Wnt pathway results in attenuation of another one. Signal transduction through Wnt5a/Ror2 causes the inhibition of canonical Wnt signaling (Mikels and Nusse 2006). In mice, Ryk promotes Wnt1 and Wnt3a-dependent gene expression. (Lu et al., 2004). Additionally, several groups reported that Ryk mediates Wnt5a-dependent axon guidance in Drosophila and mammals, as well as, Wnt11-dependent regulation of convergent extension movements in zebrafish. Thus, Ryk is proposed to play a role in canonical and non-canonical Wnt pathway. In Xenopus, PTK7 was shown to be required for PCP-dependent regulation of NC migration (Shnitsar and Borchers, 2008). Furthermore, PTK7 mediates inhibition of Wnt3a- and Wnt8-dependent canonical Wnt signaling (Peradziryi et al., 2011). Taken together, this evidence emphasizes the complexity of Wnt pathways and their mutual interactions.

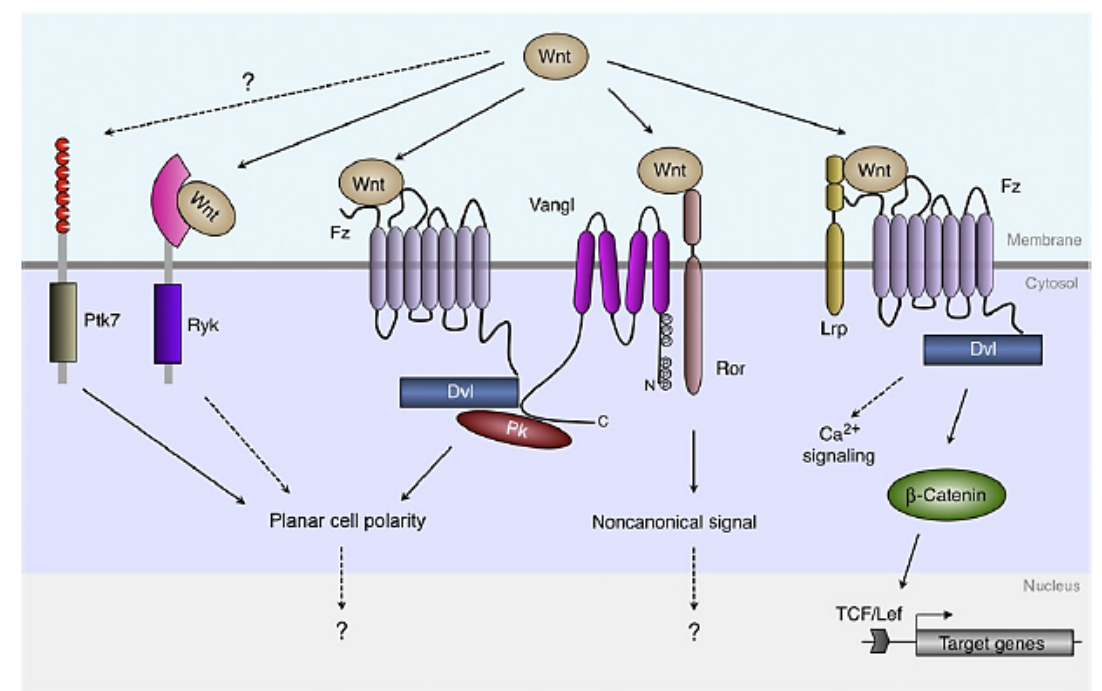

Figure.1.7 Wnt signaling pathways. Tissue-specific combination of receptors as well as involvement of Ror2, Ryk and PTK7 coreceptors results in the activation or inhibition of particular Wnt pathway. Adapted from Gao, 2012. 


\subsection{Methodes to investigate migration of NC cells in Xenopus laevis}

In vivo analysis of NC migration comprises microinjection techniques, which allow down or upregulation in gene expression through morpholino-mediated knowdown preventing protein translation, overexpression of dominant-nagative mRNA/DNA variants overwhelming endogenous gene expression and overexpression of fullfunction mRNAs/DNAs enforcing extensive expression of a particular gene. In this kind of experiment RNA/DNA constructs are injected dorsally in one blastomere at the 2-cell stage leaving the second blastomer as an internal control. Subsequently, defects in NC migration are analysed by whole mount in situ hybridization (WISH) using specific, antisense RNA probes detecting endogenous mRNAs coding for NC markers (Fig.1.7A). The advantage of this approach is the ability to monitor migration changes caused by genetic manipulations among various subpopulations of NC cells. WISH provides information about the ability of NC cells to perform migration, form NC streams as well as alternations in expression of NC markers upon manipulations. In addition, transplantation of NC cells overexpressing particular RNA/DNA constructs together with fluorescent dye into wild type embryo and vice versa allows determining if observed defect is NC cells-autonomous or if it is caused by the surrounding tissues (Fig.1.7B). Furthermore, in vitro experiment where NC cells are explanted from the embryos and cultivated on fibronectin-coated dishes, allows time-lapse monitoring of NC migration, migration behavior and formation of cell protrusion by NC cells upon overexpression of mRNAs/DNAs constructs, chemical treatment or exposure to attractant/repellent molecules (Fig.1.7C).

A
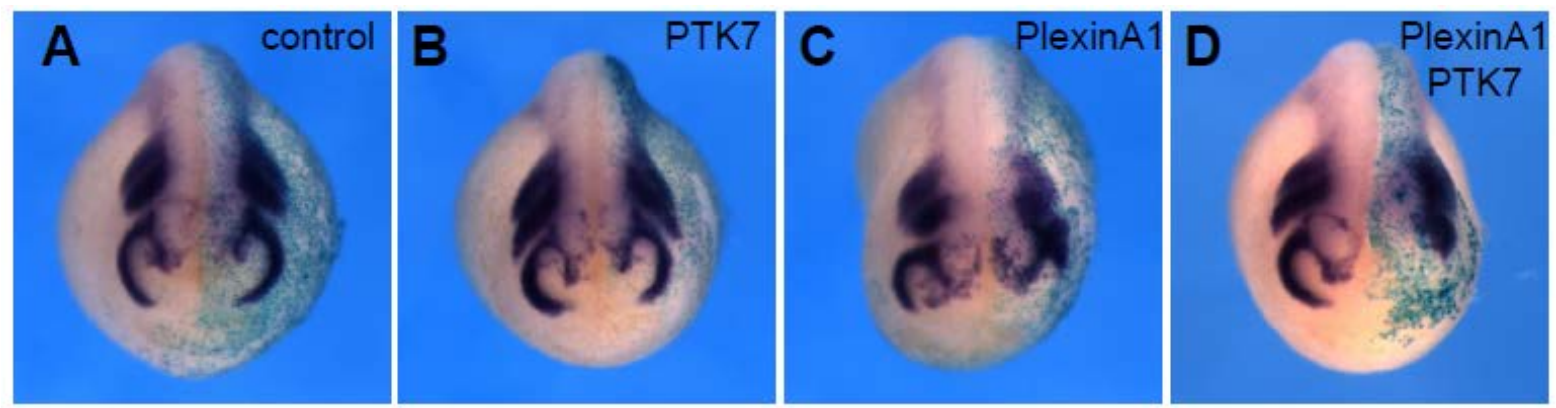
B

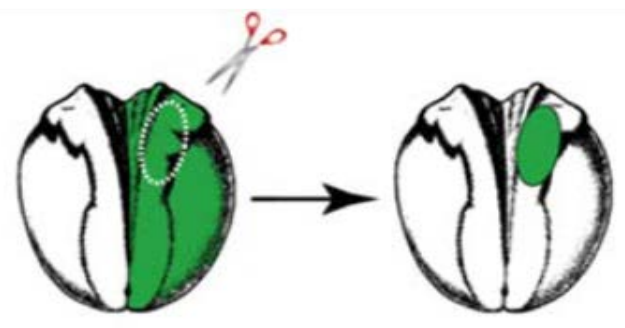

phenotyp

control
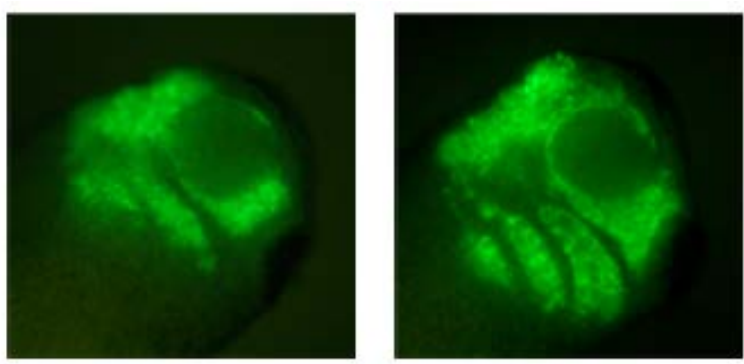

Migration behavior

C
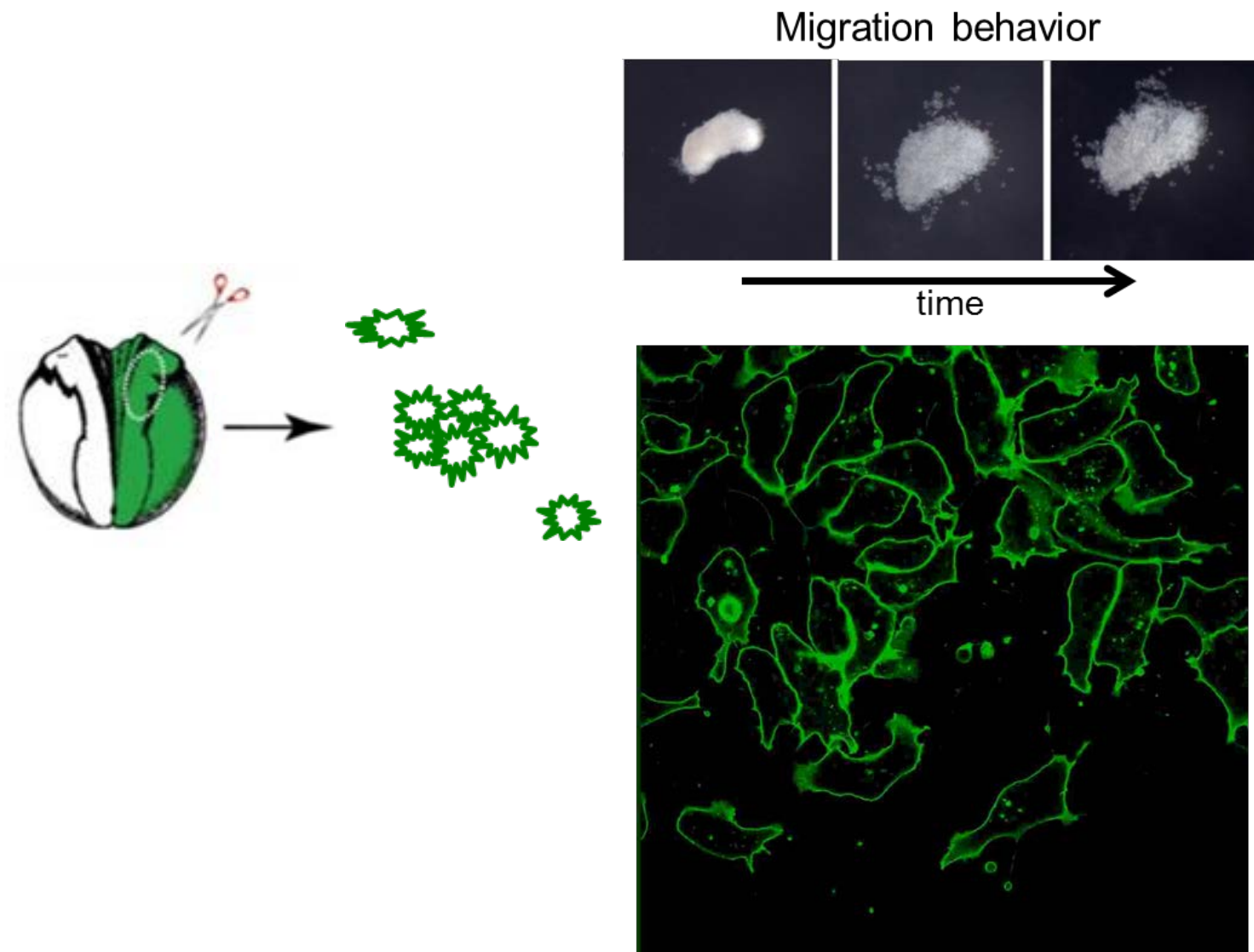

Figure.1.8. Methodes to investigate NC migration in Xenopus leavis. A Visualisation of changes in migration of Twist-positive NC population upon overexpression of RNA/DNA constructs through whole mount in situ hybridization (WISH). B Transplantation of NC cells from embryos overexpressing particular RNA/DNA constructs to wild type host and vice versa allows to determine if observed phenotyope is NC-specific or if it is caused by adjacent tissues. C Explantation of NC cells and cultivation on fibronectin-coated dishes allows life-cell imaging and analysis of migrational behavior. Adapted from Wagner et al., 2010, Shnitsar and Borchers, 2008. 


\subsection{Aims}

Canonical Wnt signaling plays a crucial role in the two phases of NC development, namely induction and differentiation. Interestingly, a potential involvement of canonical Wnt signaling in migration, which occurs after induction and precedes differentiation, remains elusive. So far, another Wnt pathway, the PCP pathway has been shown to be a key player in the orchestration of NC migration. Since both pathways share core components and several studies proved their antagonistic interactions we expect a fine-tuning of canonical Wnt signaling during migration of $\mathrm{NC}$ cells. As little is known about the potential participation of canonical Wnt signaling in post-induction NC development, we will determine the necessity of canonical Wnt signaling in migratory NC cells. Additionally, we will delineate the temporal and spatial activity of canonical Wnt signaling during NC cells migration. To study the effects of canonical Wnt signaling in migratory NC cells we will activate and inhibit canonical Wnt signaling at different time points after NC induction. Activation and inhibition of canonical Wnt signaling will be performed by whole-embryo treatment with commercially available modulators including $\mathrm{BIO}, \mathrm{LiCl}$ and IWR-1. As all chemical modulators work at the level of the cytoplasm they might exert a more global effect and not only gene expression, but also cell-cell adhesion could be affected. Therefore, modulation of canonical Wnt signaling will be expanded to overexpression of glucocorticoid-receptor (GR) fusion constructs of the Lef/Tcf transcription factors family. mRNA of GR-fusion constructs of known activatory/inhibitory activity in canonical Wnt signaling will be injected in one blastomer of 2-cell stage embryos and subsequently activated at specific time-points by Dexamethasone treatment. Alternations of $\mathrm{NC}$ migration in whole-embryos will be analyzed by in situ hybridization and RT-PCR for cranial NC markers. In addition, to in vivo experiments, migration behavior of explanted NC cells upon modulation of canonical Wnt signaling will be monitored by time-lapse imaging to asses changes in cell morphology, formation of protrusions and cell motility. Temporal and spatial activity of canonical Wnt signaling will be analyzed by monitoring nuclear localization of ß-catenin in migrating NC cells. Finally, modulation of non-canonical Wnt signaling will be performed by in vivo and in vitro treatment with commercialy available chemicals including SP600125, Box5 and IWP-2 and will allow the comparison to phenotypes observed upon modulation of canonical Wnt signaling. 


\section{Materials}

\subsection{Materials}

\subsubsection{Model organism}

In vivo and in vitro experiments were done with the use of Xenopus laevis embryos. The embryos were obtained by in vitro fertilization and staged according to Niewkoop and Faber (Niewkoop P.D., 1956). Adult frogs were provided by Nasco (Ft. Atkinson, USA).

\subsection{Chemicals, solutions, media and buffers}

\subsubsection{Chemicals}

All chemicals, used in the given work, were obtained from the following companies: Roth (Karlsruhe), Sigma (Munich), Cayman (Hamburg), Merck (Darmstadt), Life Technologies (Carlsbad, California) and Amresco (Ohio)

\subsubsection{Buffers and Solutions}

Alkaline phosphatase buffer (APB): $100 \mathrm{mM}$ Tris- $\mathrm{HCl}$ (pH 9.5), $50 \mathrm{mM} \mathrm{MgCl}$, $100 \mathrm{mM} \mathrm{NaCl}, 0.1 \%$ Tween-20

Cystein solution: 2\% L-Cystein- $\mathrm{HCl}, \mathrm{pH} 7.8$

Danylchik's for Amy (DFA) medium: $53 \mathrm{mM} \mathrm{NaCl}, 5 \mathrm{mM} \mathrm{Na} 2 \mathrm{CO}_{3}$, potassium gluconate 4,5 mM, sodium gluconate $32 \mathrm{mM}, \mathrm{MgSO}_{4} 1 \mathrm{mM}, \mathrm{CaCl}_{2} 1 \mathrm{mM}, \mathrm{BSA}_{0.1 \%}$ Ficoll: 10\% (w/v) Ficoll PM 400 (Sigma), sterile filtered Hybridization mix (Hyb-mix): 50\% (v/v) Formamid; 5xSSC; 1 mg/ml Torula RNA (Sigma); $100 \mu \mathrm{g} / \mathrm{ml}$ Heparin (Sigma); 1x Denhards; $0.1 \%$ (v/v) Tween-20; $0.1 \%$ (w/v) CHAPS (Sigma)

Injection buffer: 1x MBS, 1\% Ficoll

MAB (5x): 100 mM Maleinic acid; 150 mM NaCl, pH 7.5

MEM (10): 100 mM MOPS, 2 mM EGTA, 1 mM MgSO4

MEMFA: 1x MEM with 3.7\% Formaldehyde

PBS (10x): 8\% (w/v) NaCl, 2\% (w/v) KCl, 65 mM Na2HPO4, 18 mM KH2PO4, pH 7.4 SSC (20x): $150 \mathrm{mM} \mathrm{NaCl}, 15 \mathrm{mM} \mathrm{Na-Citrate,} \mathrm{pH} \mathrm{7.2-7.4}$

PBS/Tween: PBS (1x)/Tween 0,1\%

TAE (Tris/Acetat/EDTA) (50x): 40 mM Tris-Acetate (pH 8.5), 1 mM EDTA 
X-gal staining solution: $1 \mathrm{mg} / \mathrm{ml}$ X-gal, $5 \mathrm{mM} \mathrm{K3Fe} \mathrm{(CN)6,} 5 \mathrm{mM} \mathrm{K4Fe} \mathrm{(CN)6,} 2$ $\mathrm{mM} \mathrm{MgCl} 2$

\subsection{Other chemical substances and reagents}

Bovine fibronectin: F4759-5MG, Sigma;

DNA ladder: Low, Middle, High-Range, MBI Fermentas

6x Loading Dye, Fermentas

Human chorionic gonadotropin (HCG), Sigma

NBT and BCIP (NBT: C40H30Cl2N10O6, BCIP: C8H6NO4BrCIP x C7H9N), Roche X-gal (5-Bromo-4-chloro-3-indolyl a-D-galactopyranoside), Roche

DMSO Sigma

1,9-Pyrazoloanthrone, Anthrapyrazolone (SP600125), Sigma

N-(6-Methyl-2-benzothiazolyl)-2-[(3,4,6,7-tetrahydro-4-oxo-3-phenylthieno[3,2-

d]pyrimidin-2-yl)thio]-acetamide (IWP-2), Sigma

6-bromoindirubin-3'-oxime (BIO), Sigma and Cayman

4-[(3aR,4S,7R,7aS)-1,3,3a,4,7,7a-hexahydro-1,3-dioxo-4,7-methano-2H-isoindol-2-

yl]-N-8-quinolinyl-benzamide (IWR-1-endo), Cayman

t-Boc-NH-Met-Asp-Gly-Cys-Glu-Leu- $\mathrm{CO}_{2} \mathrm{H}$ (Box5), Calbiochem

Random Hexamers, Roche

Dexamethasome, Sigma

\subsection{Enzymes and Kits}

Illustra RNAspin Mini, GE Healthcare

GeneJET PCR purification Kit, Thermo Scientific

dNTPs 100 mM, Thermo Scientific

M-MulV Reverse Transcriptase, Thermo Scientific

Proteinase $\mathrm{K}(20 \mathrm{mg} / \mathrm{ml})$, Merck;

RNase A (100 U/ $/$ l), Sigma

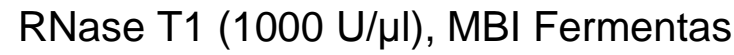

RNase Mix A (2 mg/ml)/T1(5000 U/ml), Thermo Scientific

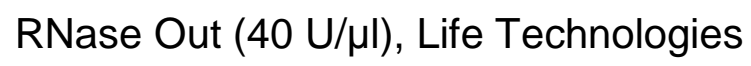

Restriction endonucleases (normal and Fast Digest), MBI Fermentas

SP6, T3, T7 RNA Polymerases (20 U/ $\mu$ l), Fermentas

RNeasy Mini Kit, Qiagen 
SP6, T3 and T7 mMESSAGE mMACHINE Kits, Ambion

Tag DNA polymerase (5 U/ $\mu \mathrm{l})$ and 10x buffer, GE Healthcare

Tag DNA polymerase (recombinant) $(5 \mathrm{U} / \mu \mathrm{l})$, 10x buffer and $25 \mathrm{mM} \mathrm{MgCl}$, Thermo

Scientific

\subsection{Laboratory equipment and software}

\section{Microinjection}

Microinjector 5242, Eppendorf

Needle puller PN-31, Narishing

\section{Equipment for the work with DNA}

Visualization and documentation of agarose gels: UV solo and P93, Biometra Nanodrop: NanoDrop 2000 Spectrophotometer, Thermo Scientific PCR-machines: Flex Cycler 4, Analytik Jena

Table centrifuge: Heraeus Pico and Fresco 17, Thermo Scientific

Water Bath: GFL 1092, GFL

Thermo Sheker: Grant Bio PCMT

\section{Optics}

Fluorescent microscope: Leica M165 FC, camera Leica DFC 450C

Confocal microscope: LSM780 with ZEN software 2011, Zeiss

Spinning Disc microscopy: Axio Observer Z1 with ZEN software 2012, Zeiss

\section{Software:}

Microsoft office 7

BLAST online system (Altschul et al., 1990)

Adobe Photoshop CS6

LAS AF Lite

LAS V4.3

Zeiss software 2011 and 2012 


\subsection{Tables}

Table 1 DNA constructs

\begin{tabular}{|c|c|c|c|}
\hline Name & Vector & Description & References \\
\hline Twist & pGEM-T & Twist & Hopwood et al., 1989 \\
\hline Sox10 & & Sox10 & Aoki et al., 2003 \\
\hline AP2 & & AP2 & Winning et al., 1991 \\
\hline Krox-20 & pGEM-T & $\begin{array}{l}\text { Krox-20, early growth } \\
\text { response protein } 2 \\
\text { EGR-2 }\end{array}$ & Wilkinson et al., 1989 \\
\hline LacZ & pCS2+ & n-ß-galactosidase & Smith and Harland, 1991 \\
\hline GAP43-GPF & pCS2+ & $\begin{array}{l}\text { membrane localized } \\
\text { GFP }\end{array}$ & Kashef et al., 2009 \\
\hline H2B mcherry & & $\begin{array}{l}\text { Momosapiens histone } \\
\text { cluster } 1 \mathrm{H} 2 \mathrm{bJ}\end{array}$ & Moriyoshi et al., 1996 \\
\hline Lef-1-GR & GR/pCS2+ & $\begin{array}{l}\text { Inducible mouse full- } \\
\text { lengh Lef1 fused to } \\
\text { human GR-LBD. }\end{array}$ & Behrens et al., 1996 \\
\hline EnR-Lef-1-GR & GR/pCS2+ & $\begin{array}{l}\text { Inducible full-length } \\
\text { Lef-1 fused to } \\
\text { Drosophila EnR and } \\
\text { human GR-LBD }\end{array}$ & Lyons et al., 2009 \\
\hline $\begin{array}{l}\text { Tcf3-VP16- } \\
\text { GR }\end{array}$ & GR/pCS2+ & $\begin{array}{l}\text { Inducible Tcf3 fused to } \\
\text { VP16 transcription } \\
\text { enhancer from } \\
\text { Herpres simplex and } \\
\text { human GR-LBD. }\end{array}$ & Borday et al., 2012 \\
\hline
\end{tabular}




\begin{tabular}{|c|c|c|c|}
\hline Tcf3 $\Delta$ C-GR & GR/pCS2+ & $\begin{array}{l}\text { Xenopus Tcf3 } \\
\text { lacking the CtBP } \\
\text { binding domain } \\
\text { fused to human GR- } \\
\text { LBD. }\end{array}$ & $\begin{array}{l}\text { Tcf3 } \triangle \text { C-GR was cloned by PCR } \\
\text { amplification from Tcf3A } \triangle \mathrm{C} / \mathrm{pCS} 2+ \\
\text { (Pukrop et al., 2001) using the } \\
\text { following primers: 5' } \\
\text { ACGAATTCATGCCTCAGCTCAA } \\
\text { 3' (forward) and 5' } \\
\text { GTCTCGAGGTTTTCCATCTCAGG } \\
\text { 3' (reverse) primers. The PCR } \\
\text { product was cut with EcoRI/Xhol } \\
\text { and inserted into the same } \\
\text { restriction sites of the GR/pCS2+ } \\
\text { vector containing the human } \\
\text { glucocorticoid receptor domain } \\
\text { (GR) (Generated by Dr. Juliane } \\
\text { Melchert, PhD Thesis: "Expression } \\
\text { screen for Wnt signaling-like } \\
\text { phenotypes identifies Fam132b as } \\
\text { a novel inhibitor of BMP signaling in } \\
\text { Xenopus") }\end{array}$ \\
\hline
\end{tabular}

Table 2 Linearization and in vitro transcription of DNA constructs

\begin{tabular}{|l|l|l|l|}
\hline Construct Name & Lin. Enzyme & Polymerase & Direction \\
\hline Twist & EcoRI & T7 & antisense \\
\hline Sox10 & EcoRI & T3 & antisense \\
\hline AP2 & HindIII & T7 & antisense \\
\hline Krox-20 & EcoRI & T3 & antisense \\
\hline Lef-1-GR & Notl & Sp6 & sense \\
\hline EnR-Lef-1-GR & Notl & Sp6 & sense \\
\hline Tcf3-VP16-GR & Notl & Sp6 & sense \\
\hline Tcf3 $\Delta$ C-GR & Notl & Sp6 & sense \\
\hline GAP43-GPF & Notl & Sp6 & sense \\
\hline H2B mcherry & Notl & Sp6 & sense \\
\hline LacZ & Notl & Sp6 & sense \\
\hline
\end{tabular}




\section{Table 3 RT-PCR primers}

All primers were ordered in the lyophilized form from Sigma Aldrich (Germany) and subsequently diluted to $100 \mu \mathrm{M}$ with HPLC-pure water (Roth).

\begin{tabular}{|c|c|c|c|}
\hline $\begin{array}{l}\text { Gene name } \\
\text { Xenopus } \\
\text { laevis }\end{array}$ & Forward and revers primer & $\begin{array}{l}\text { Product } \\
\text { length }\end{array}$ & Design \\
\hline H4 & $\begin{array}{l}\text { 5'CGGGATAACATTCAGGGTATCACT3' } \\
\text { 5' ATCCATGGCGGTAACTGTCTTCCT3' }\end{array}$ & 189bp & $\begin{array}{l}\text { Accession No. } \\
\text { X03017 }\end{array}$ \\
\hline Twist & $\begin{array}{l}\text { 5' GGGATGCAGAAAGAGGCGAT 3' } \\
\text { 5' AAGGCTTCGTTGAGGGACTG 3' }\end{array}$ & 186bp & $\begin{array}{l}\text { designed with the use } \\
\text { of NCBI Primer- } \\
\text { BLAST, NCBI } \\
\text { Reference Sequence: } \\
\text { NM_001085883.1 }\end{array}$ \\
\hline Sox10 & $\begin{array}{l}\text { 5' TCACGTTAAGCGGCCAATGA 3' } \\
\text { 5' CATGGGAGAACCATGTCGGT 3' }\end{array}$ & 349bp & $\begin{array}{l}\text { designed with the use } \\
\text { of NCBI Primer- } \\
\text { BLAST, GenBank } \\
\text { Reference Sequence: } \\
\text { AY157667.1 }\end{array}$ \\
\hline C-Jun & $\begin{array}{l}\text { 5' ACAGCAACAGCATGGGCTAT 3' } \\
\text { 5' ACAGCCACTGTTGACGTGAT 3' }\end{array}$ & 365bp & $\begin{array}{l}\text { designed with the use } \\
\text { of NCBI Primer- } \\
\text { BLAST, NCBI } \\
\text { Reference Sequence: } \\
\text { NM001090797.1 }\end{array}$ \\
\hline Brachyury & $\begin{array}{l}\text { 5' CAACTTTGGAGCCCACTGGA 3' } \\
\text { 5' TGTGTGGATTGGGCGAACAT 3' }\end{array}$ & 395bp & $\begin{array}{l}\text { designed with the use } \\
\text { of NCBI Primer- } \\
\text { BLAST, NCBI } \\
\text { Reference Sequence: } \\
\text { NM001090578.1 }\end{array}$ \\
\hline
\end{tabular}


Table 4 Antibodies and dyes

\begin{tabular}{|l|l|l|l|l|}
\hline Name & Produced in & Purpose & Dilution & Company \\
\hline anti- $\beta$-catenin & rat & IF & $1: 150$ & $\begin{array}{l}\text { generous gift of Ralph } \\
\text { Rupp }\end{array}$ \\
\hline $\begin{array}{l}\text { donkey anti-rat } \\
\text { Alexa 594 nm }\end{array}$ & donkey & IF & $1: 400$ & $\begin{array}{l}\text { Santa Cruz } \\
\text { Biotechnology }\end{array}$ \\
\hline $\begin{array}{l}\text { anti-Twist } \\
\text { Onti-rabbit } \\
488 \mathrm{~nm}\end{array}$ & gabbit & IF & $1: 50$ & Abcam \\
\hline DAPI & IF & $1: 200$ & Life Technologies \\
\hline
\end{tabular}

\section{Methods}

\subsection{DNA methods}

\subsubsection{DNA concentration measurement}

DNA quantification was performed by measurement of the absorption values at 260 nm (OD260) using the NanoDrop (2000 Spectrophotometer, Thermo Scientific). The DNA concentration in a given sample was calculated according to the following ratio: OD260 $=1$ corresponds to $50 \mu \mathrm{g}$ of dsDNA. The purity of the DNA sample was estimated by OD260/OD280 for protein contamination (optimal values: 1.6-1.8) and OD260/OD230 for salt contamination (optimal values: higher than 2,0).

\subsubsection{Agarose-gel electrophoresis}

DNA or RNA fragments were size-separated in a horizontal electrical field being embedded into agarose-matrix. $1 \%(\mathrm{w} / \mathrm{v})$ agarose gels were prepared in $1 \mathrm{x}$ TAE buffer. Gels always contained $0.5 \mu \mathrm{g} / \mathrm{ml}$ ethidium bromine to visualize nucleic acids. Before loading samples into gel slots, nucleic acids were mixed with $5 / 1(\mathrm{v} / \mathrm{v})$ of DNA loading dye (6x, Fermentas). The electrophoresis was run in the 1x TAE running buffer at 100-120 V in a house made horizontal electrophoresis chamber (Sharp et 
al., 1973). After electrophoresis, DNA bands were visualized with UV-transilluminator and documented with UV solo (Biometra). The DNA fragments were sized according to the standard DNA ladder run in parallel (High, Middle or Low Range, Fermentas)

\subsubsection{DNA restriction digest}

For the digest of plasmid DNA, 0.5-1 $\mu \mathrm{g}$ of the DNA sample was incubated with 2-10 $U$ of a chosen enzyme in the corresponding buffer. The total reaction volume was 25 $\mu \mathrm{l}$, and the digest was performed for $1-12 \mathrm{~h}$ at $37^{\circ} \mathrm{C}$.

\subsection{RNA methods}

In order to avoid the RNA degradation by RNAses, the work with RNA samples was performed exclusively with RNAse-free water (Amresco)

\subsubsection{Total RNA extraction, cDNA synthesis and RT-PCR}

For expression analysis of embryos injected with GR-inducible constructs embryos were injected with $25 \mathrm{pg}$ of Tcf3A $\triangle \mathrm{C}-\mathrm{GR}, 25 \mathrm{pg}$ of Tcf3-VP16-GR, $25 \mathrm{pg}$ of Lef-1-GR and $25 \mathrm{pg}$ of EnR-Lef-1-GR into two blastomers of a 2-cell stage embryo, cultivated till premigratory stages (16-18) and treated with Dexamethasome (4 $\mathrm{gg} / \mathrm{ml})$. For extraction of total RNA 10 embryos from each injection pool were fixed in $\mathrm{N}_{2}$ liq at stage 24/25 and macerated in 720 $\mu$ I TRIzol ${ }^{\circ}$ Reagent (Life Technologies) using a sterile Omnican ${ }^{\circledR} 40$ syringe (Braun). Lysates were incubated for $10 \mathrm{~min}$ (RT). Subsequently $144 \mu \mathrm{l}$ of Chloroform (Roth) were added to the samples followed by 5 min of incubation (RT) and 20 min of centrifugation $\left(4^{\circ} \mathrm{C}, 13000 \mathrm{rpm}\right)$. The upper phase was transferred into a new tube, 1 vol. of Chloroform (Roth) was added to the samples followed by $10 \mathrm{~min}$ of centrifugation $\left(4^{\circ} \mathrm{C}, 13000 \mathrm{rpm}\right)$. Again the upper phase was transferred into a new tube and precipitation of nucleic acids was performed by addition of $360 \mu \mathrm{l}$ of 2-Propanol (Roth) and overnight incubation ($20^{\circ} \mathrm{C}$ ). After $30 \mathrm{~min}$ of centrifugation $\left(4^{\circ} \mathrm{C}, 13000 \mathrm{rpm}\right)$ pellet was washed with $500 \mu \mathrm{l}$ of $75 \%$ ethanol by centrifugation for $5 \mathrm{~min}\left(4^{\circ} \mathrm{C}, 13000 \mathrm{rpm}\right)$. The air-dried pellet was dissolved in $20 \mu \mathrm{l}$ of RNase-free water. DNA digestion was performed for $1 \mathrm{~h}$ at $37^{\circ} \mathrm{C}$ (1 $\mu$ l DNase TURBO (Ambion Kit), 0,5 $\mu$ l Rnase Out (Life Technologies), $10 \mu \mathrm{l} 5 \mathrm{x}$ transcription buffer (Thermo Scientific), filled up with $\mathrm{H} 2 \mathrm{O}$ to $50 \mu \mathrm{l}$ ). DNase treatment 
was stopped by addition of $150 \mu \mathrm{l}$ RNase-free water. Subsequently 1 vol. of Roti@Aqua-Phenol/C/l (Roth) was added to the samples followed by $10 \mathrm{~min}$ of centrifugation $\left(4^{\circ} \mathrm{C}, 13000 \mathrm{rpm}\right)$. The upper phase was transferred into a new tube, 1 vol. of Chloroform/lsoamylalkohol (24:1) (Merck/Roth) was added to the samples, followed by $10 \mathrm{~min}$ of centrifugation $\left(4^{\circ} \mathrm{C}, 13000 \mathrm{rpm}\right)$. The upper phase was again transferred into a new tube and another precipitation was performed by addition of $0.2 \mathrm{vol}$. of $5 \mathrm{M}$ ammonium acetate and 1 vol. of 2-Propanol (Roth) and overnight incubation $\left(-20^{\circ} \mathrm{C}\right)$. After $30 \mathrm{~min}$ of centrifugation $\left(4^{\circ} \mathrm{C}, 13000 \mathrm{rpm}\right)$ the pellet was washed with $75 \%$ ethanol by centrifugation for $5 \mathrm{~min}\left(4^{\circ} \mathrm{C}, 13000 \mathrm{rpm}\right)$, air-dried and dissolved in $30 \mu$ of RNase-free water.

\subsubsection{Revers transcription and RT-PCR}

cDNA synthesis was performed according to the manufacturer's instructions ( $M$ MuLV Reverse Transcriptase, Protocol for First Strand cDNA Synthesis, Thermo Scientific). RNA concentration was adjusted to $1 \mu \mathrm{g}$, hexamers concentration was adjusted to $0,2 \mu \mathrm{g}(100 \mathrm{pmol})$. The total volume of reaction was $11,5 \mu \mathrm{l}$. An optional step for GC rich templates was included. Samples were mixed gently, centrifuged brifly and incubated for $5^{\prime}$ at $65^{\circ} \mathrm{C}$. Samples were placed chilled on ice, centrifuged briefly and placed back on ice. Subsequently, samples were mixed with following components: $4 \mu \mathrm{l}$ 5x RT reaction buffer (Thermo Scientific), 0,5 $\mu \mathrm{l}$ (20U) RNase Out (Life Technologies), $2 \mu \mathrm{l}(1 \mathrm{mM}$ final concentration) dNTPmix 10mM each (Thermo Scientific), $2 \mu \mathrm{l}(40 \mathrm{U}) \mathrm{M}-M u L V$ Reverse Transcriptase. The total volume of reaction was $20 \mu \mathrm{l}$.

To amplify desired cDNA fragments, a standard PCR reaction was used, which contains the following components: $2,5 \mu \mathrm{l} 10 \mathrm{x}$ buffer (GE healthcare), $0,5 \mu \mathrm{lNTP}$ mix $10 \mathrm{mM}$ each (Thermo Scientific), 0,5 $\mu \mathrm{l}$ each forward and revers primer (Sigma), $0,25 \mu \mathrm{l}$ Tag Polymerase (GE healthcare), $1 \mu \mathrm{l}$ cDNA and filled up to $25 \mu \mathrm{l}$ with $\mathrm{H} 2 \mathrm{O}$. Or according to protocol of Tag DNA polymerase (recombinant) (Thermo Scientific): 2,5 $\mu \mathrm{l}$ 10x Tag buffer with $\mathrm{KCl}$ (Thermo Scientific), 0,125 $\mu \mathrm{l}$ Tag Polymerase (Thermo Scientific), 0,5 $\mu \mathrm{l}$ dNTP mix $10 \mathrm{mM}$ each (Thermo Scientific), 0,5 $\mu \mathrm{l}$ each forward and revers primer (Sigma), $1,5 \mu \mathrm{l}$ of $25 \mathrm{mM} \mathrm{MgCl}_{2}$ (Thermo Scientific), $1 \mu \mathrm{l}$ CDNA and filled up to $25 \mu \mathrm{l}$ with $\mathrm{H} 2 \mathrm{O}$. The initial DNA denaturation step was performed for $5 \mathrm{~min}$ at $95^{\circ} \mathrm{C}$, and all subsequent DNA denaturation steps for $30 \mathrm{sec}$ at $95^{\circ} \mathrm{C}$. The 
annealing step was done for $30 \mathrm{sec}$ at $58^{\circ} \mathrm{C}$. The elongation step was done for 30 sec at $72^{\circ} \mathrm{C}$. The one PCR cycle included 27 loops. The final elongation step was done for $10 \mathrm{~min}$ at $72^{\circ} \mathrm{C}$ (Mullis et al., 1986).

\subsubsection{In vitro transcription}

\subsubsection{In vitro transcription of the dioxigenin-labelled antisense RNA for the whole mount in situ hybridization (WISH)}

For the synthesis of a digoxigenin-labelled antisense WISH RNA probe the following reaction mixture was set up:

$5 \mu$ of a $5 x$ transcription buffer

$0,5 \mu \mathrm{l}$ of pyrophosphatase $4 \mathrm{U} / \mathrm{mol}$ (Fermentas)

4 Il DIG mix (Stock solutions: Digoxigenin-11-UTP (250 nmol)(Roche), ATP, GTP, CTP (100 mM each, diluted 1:10)(Fermentas), UTP single (100 mM, diluted 1:10) (Fermentas). Mix: $25 \mu \mathrm{l}$ Digoxigenin-11-UTP (10 mM), $44 \mu \mathrm{l}$ UTP (10 mM), $69 \mu \mathrm{l}$ ATP, CTP, GTP (10 mM))

$1 \mu \mathrm{l} 0.75$ M DTT

$1 \mu$ l RNAseOut $(40 U / \mu l)$

$200 \mathrm{ng}$ linearized plasmid (the DNA template)

$1 \mu \mathrm{l}$ corresponding polymerase (T3, T7 or Sp6)

RNAse-free water to the final volume of $25 \mu \mathrm{l}$

The reaction was carried out for $2 \mathrm{~h}$ at $37^{\circ} \mathrm{C}$, and then the DNA template was destroyed by incubation with $1 \mu$ l of the TURBO DNase $(2 \mathrm{U} / \mathrm{ml})$ (mMESSAGE mMACHINE Kits Ambion) for $15 \mathrm{~min}$ at $37^{\circ} \mathrm{C}$. The obtained WISH probe was purified from the reaction mixture with RNeasy Mini Kit (Quiagen) according to manufacturer's instructions.

\subsubsection{In vitro transcription of capped-mRNA for microinjections}

The synthesis of mRNA for microinjections was done with Sp6, T7 or T3 mMESSAGE mMACHINE Kits (Ambion) according to manufacturer's instructions. 
The reaction was set up in a volume of $20 \mu \mathrm{l}$ and 0.5-1 $\mu \mathrm{g}$ of the linear DNA was used for 1 reaction ( $6 \mu$ linearized plasmid, $10 \mu$ NTP/Cap, $2 \mu$ l buffer, $2 \mu \mathrm{l}$ enzyme). The in vitro transcription was carried out for $2 \mathrm{~h}$ at $37^{\circ} \mathrm{C}$, and subsequently the template was destroyed by 15 min DNase treatment. The mRNA was purified with RNeasy Mini Kit (Qiagene).

\subsubsection{RNA analysis and concentration measurement}

The RNA samples, both DIG-labelled and capped-mRNA, were analyzed on the $1 \%$ agarose gel supplemented with $0.5 \%$ ethidium bromide. The concentration of the total RNA and capped-mRNA was measured using the NanoDrop (2000 Spectrophotometer, Thermo Scientific), by using the following ratio: OD260=1 corresponds to $40 \mu \mathrm{g}$ of the RNA. Similar to DNA, the RNA purity was analyzed by measurement of OD260/OD280 and OD260/OD230 ratios. Additionally, the amount of DIG-labeled RNA was estimated visually on an agarose gel.

\subsection{Xenopus laevis embryos injections and manipulations}

\subsubsection{Preparation of Xenopus laevis testis}

The testis was removed from a narcotized decapitated male frog, washed with and stored in $1 \times$ MBS buffer at $4^{\circ} \mathrm{C}$

\subsubsection{Injections and culture of Xenopus laevis embryos}

Embryos were obtained from Xenopus laevis female frogs by HCG induced egglaying (800 -1000 U HCG approximately 12 hours before egg-laying). Spawns were in vitro fertilized with minced testis in $0.1 \mathrm{XMBS}$, dejellied with $1.5-2 \%$ cyteine hydrochloride, $\mathrm{pH} 8.2$ and cultured in $0.1 \times \mathrm{MBS}$ at $12.5-18^{\circ} \mathrm{C}$. Injections were performed in the injection buffer on a cold plate $\left(12.5^{\circ} \mathrm{C}\right)$. The mRNAs were injected animally to the prospective ectodermal tissues. For all purposes 2-, 4-cell injections were done. The injection volume was adjusted to 2 or $4 \mathrm{nl}$. Injected embryos were kept for at least 1 hour in the injection buffer on a cooling plate $\left(12,5^{\circ} \mathrm{C}\right)$ and then 
transferred into $0.1 \mathrm{x} \mathrm{MBSH}$. The staging of embryos was done according to Niewkoop and Faber (1956).

\subsubsection{Functionality of GR-inducible constructs in canonical Wnt pathway}

Activity and hormone inducibility of GR-inducible constructs was verified by their function in canonical Wnt signaling. Each construct was determined as activator or inhibitor of canonical Wnt pathway by analyzing its effect on primary dorsalventral body axis formation. RNAs of candidate activating constructs - like Lef1- GR and Tcf3-VP16 - were injected marginally into one ventral blastomere at the 4-cell stage. Embryos were cultured with Dexamethasone (Sigma) starting at the 16-cell stage (the solution was changed to normal medium at gastrula stages 10-11) and induction of double axis was analyzed at early tailbud stages. RNAs coding for candidate inhibiting constructs - like EnRLef1-GR or Tcf3 $\triangle C-G R$ - were injected into both dorsal blastomeres at the 4-cell stage, treated with Dexamethasone starting at the 16-cell stage (the solution was changed to normal medium at gastrula stages 10-11) and ventralized embryos were scored at early tailbud stages (Most work was done by Dr. Juliane Melchert, PhD Thesis: "Expression screen for Wht signaling-like phenotypes identifies Fam132b as a novel inhibitor of BMP signaling in Xenopus").

\subsubsection{In vivo and in vitro analysis of NC migration}

For analysis of NC migration RNAs of Tcf3 $\triangle C-G R$, Tcf3-VP16-GR, Lef-1-GR (50 pg for migration analysis in whole embryos, $75 \mathrm{pg}$ for life-cell imaging analysis of explanted NC cells), were co-injected with $30 \mathrm{pg}$ GAP43-GFP and $150 \mathrm{pg} \mathrm{H} 2 \mathrm{~B}$ mcherry or $50 \mathrm{pg}$ of lacZ RNA into one blastomere of a 2-cell stage embryo. Embryos at stage 16/17 exhibiting GFP- and mcherry-fluorescence were sorted in terms of left or right side fluorescence and used for explanation. Embryos co-injected with $50 \mathrm{pg}$ lacZ RNA were treated with Dexamethasome (Sigma) $(4 \mu \mathrm{g} / \mathrm{ml})$ at premigratory (1618) or migratory NC cell stages (20-21), further cultivated until stages 22-24 (Sox10) or 25-29 (Twist) and analyzed by whole mount in situ hybridization. $\beta$-galactosidase staining and whole mount in situ hybridization were performed as described (Harland, 1991; Borchers et al., 2001). Antisense probes were synthesized from the published plasmids listed in table 2.1. For chemical treatment embryos at premigratory (16- 
18/19) or migratory NC cell stages (20-21) were transferred to $1 \times$ MBS medium containing 6-bromoindirubin-3'-oxime (BIO) $(12,5 \mu \mathrm{M}, 25 \mu \mathrm{M})$ or IWR-1 $(10 \mu \mathrm{M}, 20$ $\mu \mathrm{M}, 100 \mu \mathrm{M})$ or DMSO as solvent control, cultivated until stage 22-29 and analyzed using whole mount in situ hybridization.

\subsubsection{Xenopus NC explants for life-cell imaging}

For in vitro life-cell imaging cranial NC cells (CNC) from embryos co-injected with GR-inducible constructs and GAP43-GFP/H2B mcherry RNA, were explanted at stage $16 / 17$. The operation took place in petri dishes coated with $1 \%$ agarose in $0,8 x$ MBS. Prior to explantation, the embryonic vitelline membrane was manually removed and the embryo was immobilized by placing it in a rectangular hole cut out of the agarose coating. A small piece of epidermis covering the cranial NC area was peeled off from the GFP/mcherry-positive side. The underlying NC cells were removed, divided into smaller pieces and placed on fibronectin-coated chamber slides (LabTek ${ }^{\circledR}$ Chamber Slide, Thermo Scientific) containing DFA medium with diluted reagents. Chamber slides were washed once with pure DFA before applying DFA with reagents. For inducing GR-constructs, Dexamethasone was used at the concentration of $20 \mu \mathrm{g} / \mathrm{ml}$ was used. For chemical treatment concentration of $1 \mu \mathrm{M}$ BIO, 2,5 $\mu \mathrm{M}$ IWR1, $1 \mu \mathrm{M}$ IWP-2, $1 \mu \mathrm{M}$ SP600125 and $100 \mu \mathrm{M}$ Box5 in DFA was used. The respective concentrations of ethanol or DMSO were used as solvent control. Explants were cultured in the respective media directly after explantation and time-lapse imaging was started on average 2-3 hours after preparation of explants representing stage 19/20 of control embryos from the same batch. Time-lapse analysis of NC cell migration was performed using Spinning Disk microscopy (Axio Observer Z1 with ZEN software 2012 (Zeiss)). For data analysis membrane tethered GFP (GAP43- GFP) was used to assess the general morphology of explanted NC cells, their ability to form protrusions, and their interactions with other NC cells. Histon 2B tagged with mcherry marked cell nuclei and allowed tracking of individual NC cells. 


\subsubsection{4 ß-catenin localization in Xenopus NC explants}

To analyze the nuclear localization of ß-catenin cranial NC cells were explanted as described above. Explanted NC cells were placed on a fibronectin-coated chamber slide (Lab-Tek ${ }^{\circledR}$ Chamber Slide, Thermo Scientific) filled up with DFA medium containing $1 \mu \mathrm{M}$ BIO or DMSO as a solvent control and cultivated for 30 minutes, 3 hours or 7 hours at $16^{\circ} \mathrm{C}$ on a cooling plate. After cultivation the medium was removed. Cranial NC cells were covered with a cover slide attached with Vaseline and fixed with MEMFA for 20 minutes. Explants were washed 3x 10' in Ptw and blocked overnight with $1 \%$ BSA or $10 \%$ FCS in Ptw. Next day bloking solution was removed and explants were rinsed once with Ptw and primary antibodies diluted in Ptw with $1 \%$ BSA or $10 \%$ FCS were applied overnight. Next day primary antibodies were removed, explants were washed 3x 10' with Ptw with 1\% BSA or 10\% FCS and secondary antibodies diluted in Ptw with 1\% BSA or 10\% FCS were applied for 2-3h. Subsequently, secondary antibodies were removed, explants were washed 3x'10 with Ptw with 1\% BSA or 10\% FCS, stained with DAPI diluted in PBS for 7', washed $2 \times 10$ ' with PBS. Antibodies were used in the following dilutions: rat anti- $\beta$-catenin (generous gift of Ralph Rupp) 1:150, donkey anti-rat Alexa 594 nm (Santa Cruz Biotechnology) 1:400, rabbit anti-Twist (Abcam) 1:50, goat anti-rabbit Oregon Green $488 \mathrm{~nm}$ (Life Technologies) 1:200, DAPI (Carl Roth) 1 $\mu \mathrm{g} / \mathrm{ml}$. After immunostaining cranial NC cells were imaged using a confocal microscope (LSM780 with ZEN software 2011 (Zeiss)). The area of each nucleus in the NC explants was determined by DAPI staining and the intensity of the ß-catenin fluorescent signal expressed as Integrated Density was measured using ImageJ. Integrated Densities of ß-catenin fluorescent signals from all explants of a particular treatment were averaged. One Way ANOVA and post hoc Games-Howell test were used for statistical analysis.

\subsection{Image Analysis}

Image analysis and data evaluation was performed in collaboration with $\mathrm{Dr}$ Timo Aspelmeier (Institute for Mathematical Stochastics and Felix Bernstein Institute for Mathematical Statistics, Georg August University, Göttingen) and Lutz Künneke (Institute for Theoretical Physics, Georg August University, Göttingen). The description of methods was done by Dr Timo Aspelmeier. 
Image analysis was performed using a custom filtering routine in Matlab 2010b, adapted from the one described in Kanesaki et al. (Kanesaki et al., 2011). Images were presmoothed by convolution with a gaussian kernel with a width of 2-3 pixels. The typical inverse diameter of the fluorescently labeled nuclei was approximated by the position Kmax of the dominant mode of the radial averaged power spectral density (PSD), and this position was estimated by the first moment of the PSD. Contributions to the Fourier transform of the image from wavevectors both much smaller and much larger than Kmax were removed by multiplication of the Fourier transform with a gaussian window function with variance $K$ max2/8 centered around Kmax (a gaussian window function was used rather than a box-like window function to minimize artifacts). This serves to remove both high frequency image content (noise) and low frequency content (slow variations in background intensity). An inverse Fourier transform was then applied to the modified Fourier transform to obtain a filtered image showing only the nuclei against a homogeneous background. The images were then binarized by thresholding, and image pixels containing a " 1 " were assumed to belong to a nucleus. A suitable threshold was found to be the $80 \%$ quantile of the gray value distribution of the image. Single isolated "0" pixels inside a cluster of "1" pixels were removed by the operation of image opening (Soille, 2003). The resulting segmentation was verified by eye for some exemplary cases. The cell positions were then extracted from the images by calculating the center of mass of each connected cluster of pixels belonging to a nucleus (see Savin and Doyle (Savin and Doyle, 2005) for a discussion of particle tracking errors). These positions were tracked over the image sequence, i.e. for each pair of consecutive images each nucleus in the first image was identified with a nucleus in the second image in such a way that the total squared movement between the images was minimized. Sometimes nuclei are lost by going out of the focal plane or crossing the image border, or new ones may appear for similar reasons. Hence tracking of nuclei is not failsafe. Therefore we discarded tracks with very large jumps between consecutive images (more than half the mean nearest neighbor distance) or of very short length (2-3 images). 


\subsubsection{Data evaluation- cell classification}

To allow for a structural characterization of the cell configurations, cells were classified as center, border, or outlier cells in the following way. In each frame the Delaunay triangulation of cell positions was calculated. Connected cells were treated as neighbors. The mean distance between neighbors in the first frame was denoted as r0. Each cell, which had all of its neighbors at a distance of $\mathrm{r} 0$ or less away, was classified as a center cell. Cells with at least one neighbor further away than ro but with at least one center cell neighbor were classified as border cells. All remaining cells were classified as outlier cells. The concept is illustrated in Fig. 5C.

\subsubsection{Data evaluation-mean cluster size}

To characterize the tendency of cells to stick together, we partitioned the cells into clusters. A cluster is defined as a maximal set of neighboring cells connected by distances $\leq \mathrm{r} 0$. We define the "size" of a cluster as the number of cells in the cluster. At each time we can determine the mean cluster size, which is a measure of how much the cells have moved apart: a mean cluster size of the order of the total number of cells means that almost all cells form one big cluster, whereas a mean cluster size of the order of 1 means that most cells have separated from each other and each one forms its own cluster.

\subsubsection{Data evaluation-mean square displacement}

The previous concepts of cell classification and cluster sizes describe static (i.e. single time) properties of the cell assemblies. In order to characterize dynamic properties, i.e. movement of cells over several points in time, we used the mean square displacement where $\mathrm{r} \rightarrow(\mathrm{t})$ denotes the position of cell $\mathrm{i}$ at time $\mathrm{t}$ and $\mathrm{N}$ is the total number of cells. For cells undergoing directed motion this quantity grows quadratically in time, whereas for cells fluctuating around a fixed point in space it approaches a limit. The intermediate case of diffusive motion shows a linear increase in time. For a review of applications of the mean square displacement in living cells see Gal et al. (Gal et al., 2013).

$$
\Delta r^{2}(t):=\frac{1}{N} \sum_{i=1}^{N}\left(\vec{r}_{i}(t)-\vec{r}_{i}(0)\right)^{2}
$$




\section{Results}

\subsection{Activation of canonical Wnt signaling by BIO and treatment causes defects in migration of NC cells}

To analyze the role of canonical Wnt signaling in migration of cranial NC cells, 6bromoindirubin-3'-oxime (BIO), an inhibitor of GSK3ß was used (Meijer et al., 2003; Myers et al., 2014) to specifically activate canonical Wnt signaling during migration of NC cells. Embryos were treated with BIO either at premigratory stages (16-18), where NC cells were already induced but had not migrated yet, or at early migratory stages (20-21) (Fig.4.1.1). BIO was added to the culture media and embryos were kept in this solution till fixation. NC migration upon treatment was analyzed at tadpole stages by in situ hybridization using the NC marker Twist. Twist is expressed already in NC precursor cells and remains to be expressed as the cranial NC cells migrate through the branchial arches (Lander et al., 2013), thus is an excellent marker to monitor NC migration throughout all stages. Treatment of Xenopus embryos with BIO at premigratory NC cell stages caused three types of defects in NC migration (Fig. 4.1.2A). In the first, most frequent phenotype embryos showed a strong inhibition of migration. Additionally, a severe reduction of Twist-positive cells was observed. A small cluster of Twist-positive NC cells remained in the premigratory position next to neural tube and only NC cells of the mandibular branch reached the final location surrounding the developing eye (phenotype: 'inhibited migration' Fig. 4.1A, panel IV, red arrowheads; Fig. 4.1.2B, red bars). In the second, less severe phenotype, cranial NC cells were separated into the typical branches, but the Twist-positive cells were also reduced and their migration was inhibited (phenotype: 'reduced branches' Fig. 4.1A, panel III, yellow arrowheads; Fig. 4.1.2B, yellow bars). In the third, the least severe phenotype, NC cells showed the typical branch-like structure but the posterior NC streams seemed to be merged and their migration was also inhibited (phenotype: 'merged branches' (Fig. 4.1.2A, panel II, green arrow; Fig. 4.1.2B, green bars). To sum up, the inhibition of migration was less severe for the 'reduced branches' and 'merged branches' phenotypes in comparison to 'inhibited migration' phenotype. BIO treatment at migratory stages caused the same defects, however, most embryos showed the less severe 'reduced branches' phenotype (Fig. 4.1.2C, panel III; yellow arrowheads; Fig. 4.1.2D, yellow bars). Similarly to treatments at premigratory stages, 
the separation of the cranial NC cells into branches in this phenotype was not disrupted, but a reduction of the Twist-expressing cells was again observed. In addition, the embryos treated at migratory stages also showed the 'merged branches' and 'inhibited migration' phenotype. Taken together, BIO treatment at premigratory and migratory stages produced comparable results, however, these were less severe if embryos were treated at migratory stages.

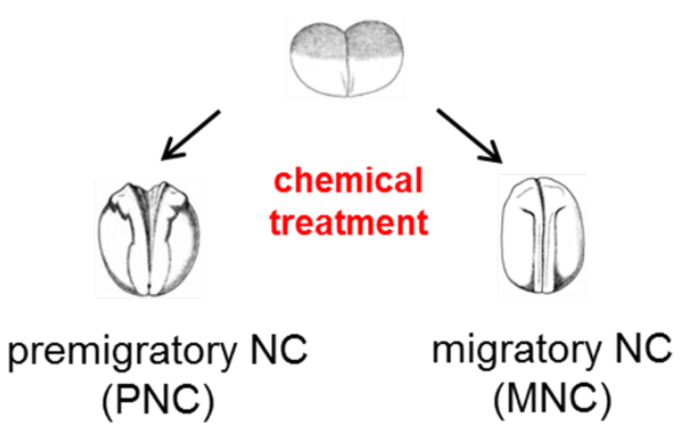

Figure.4.1.1.Experimental scheme. Wild type Xenopus embryos were treated with particular chemical modulator at premigratory or migratory NC stages. DMSO served as a solvent control. The given scheme was applied in each experiment using chemical modulators. 
A

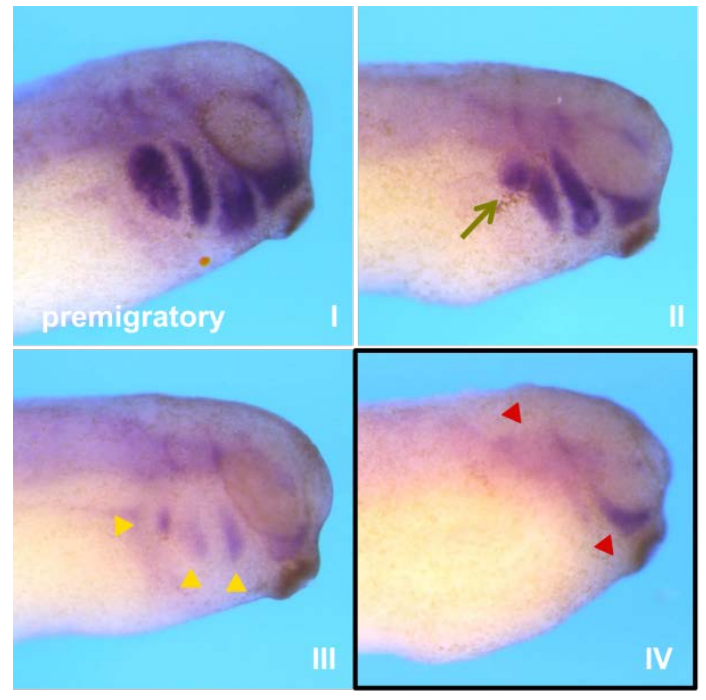

B

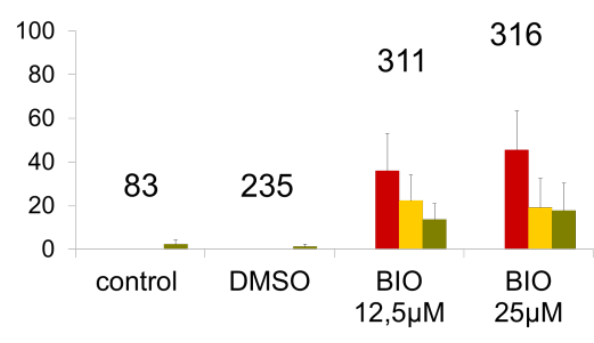

C

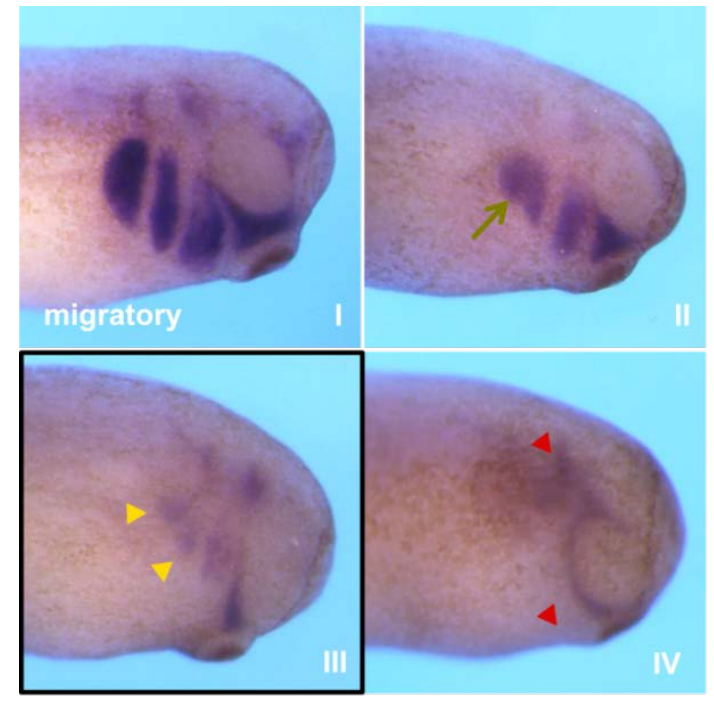

D

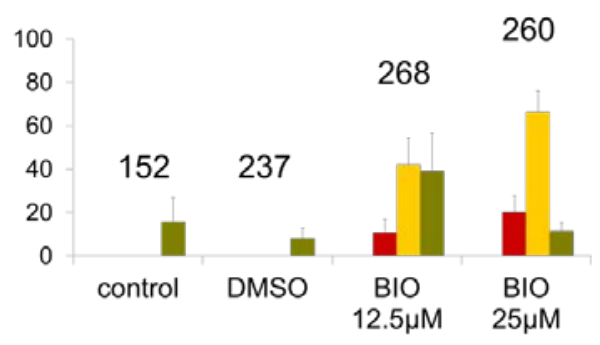

no NC

inhibited miogration

reduced branches

merged branches

Figure 4.1.2. Activation of canonical Wnt signaling using BIO at premigratory and migratory NC cell stages affects NC migration. Wild type Xenopus embryos were treated with BIO at NC premigratory or migratory stages or with DMSO as a solvent control. A The panel shows the different phenotypes observed after BIO treatment at premigratory stages. Phenotypes are grouped by increasing severity: unaffected embryo (I), embryo with merged NC branches (II), embryo with reduced NC branches (III), embryo with inhibited NC migration and reduced Twist expression (IV). Green arrow marks merged posterior NC streams. Yellow arrows mark reduced NC branches. Red arrows mark a weakly stained NC cluster in the premigratory position and the remaining mandibular branch. The black frame marks the most frequent phenotype. B Graph summarizing the percentage of phenotypes observed in 5 independent experiments. Standard errors of the mean and number of treated embryos are indicated for each column. C The panel shows the different phenotypes observed after BIO treatment at migratory stages. Phenotypes are grouped by increasing severity as for the premigratory treatment. D Graph summarizing the percentage of phenotypes observed in 3 independent experiments. Standard errors of the mean and number of treated embryos are indicated for each column. 


\subsection{Functionality of Lef1/Tcf 3 constructs in double axis assay}

BIO increases ß-catenin levels in the cytoplasm by inhibiting GSK3ß and can potentially influence other intercellular processes. Since Lef/Tcf transcription factors are the most downstream targets of canonical Wnt signaling, the inducible constructs of these proteins were used to compare phenotypes observed upon BIO treatment. Prior to investigating the effect of Lef/Tcf modulation on NC migration, the functionality of the respective Tcf/Lef glucocorticoid-receptor (GR)-fusion constructs was assessed by analyzing their ability to induce a secondary axis in Xenopus embryos (Supplement Fig.6.1) (tested mostly by Dr. Juliane Melchert-PhD Thesis: Expression screen for Wnt signaling-like phenotypes identifies Fam132b as a novel inhibitor of BMP signaling in Xenopus). Among many constructs tested only Lef1-GR and Tcf3-VP16-GR were able to activate canonical Wnt signaling in double axis assays, therefore these constructs were used to analyze how Lef/Tcf signaling affects NC migration (Fig.4.2).

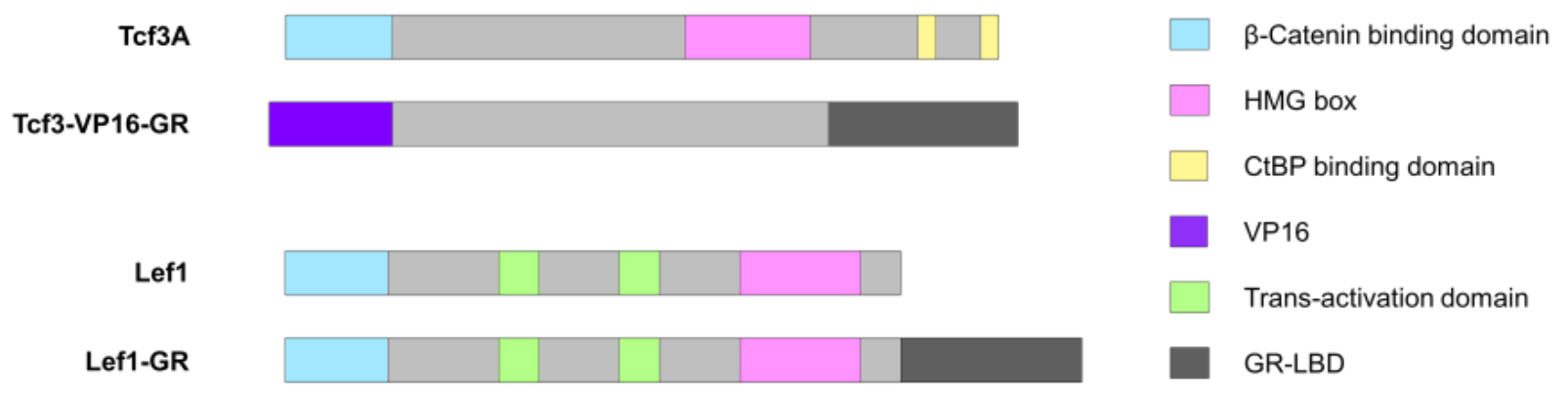

Figure 4.2. Lef-1-GR and Tcf3-VP16-GR activate canonical Wnt signaling in early Xenopus embryos as indicated by secondary axis induction A Schematic representation of wild type and modified GR-fusion constructs of Tcf3 and Lef1 used for activation of canonical Wnt signaling. Tcf3-VP16-GR is a construct, where the ß-catenin binding domain was replaced with the VP16 transcriptional activator (Vonica et al., 2000; Agathocleous et al., 2009; Borday et al., 2012).

\subsection{Activation of Lef1/Tcf3 signaling causes the same defects as BIO treatment}

To activate canonical Wnt signaling in migrating NC cells by overexpressing Lef1-GR or Tcf3-VP16-GR, mRNA of each construct was injected in one blastomere of a 2-cell stage embryo together with lacZ RNA for lineage tracing (Fig.4.3.1). The uninjected 
blastomer served as an internal control allowing comparison of NC migration in the same embryos. The respective fusion-proteins, blocked by the heat shock proteins in the cytoplasm, were activated at premigratory (16-18) and early migratory stages (2021) by Dexamethasone treatment, which allowed for their translocation into the nucleus. Alternations in NC migration were analyzed by Twist in situ hybridization at late migratory stages (25-29). Overexpression of Lef-1-GR caused two types of defects, which were also classified according to their severity. Similarly to BIO treatment, activation of Lef1-GR at premigratory NC stages lead mostly to an inhibition of NC migration and a reduction of Twist-expressing cells. The majority of embryos showed strongly inhibited NC migration where clusters of NC cells were remaining at the premigratory position. Only the mandibular branch was present at its final location surrounding the developing eye ('inhibited migration' phenotype: Fig. 4.3.2E, upper panel, red arrowheads; Fig. 4.3.2F, red bar). Less frequently the activation of the Lef1-GR construct caused the less severe 'reduced branches' phenotype, where the NC branches were more narrow and/or shorter and the most posterior stream was either missing or appeared diffuse ('reduced branches' phenotype: Fig. 4.3.2E, lower panel, yellow arrowheads, Fig. 4.3.2F, yellow bars). If the Lef1-GR construct was activated at migratory NC stages the same phenotypes were observed (Fig. 4.3.2G,H).

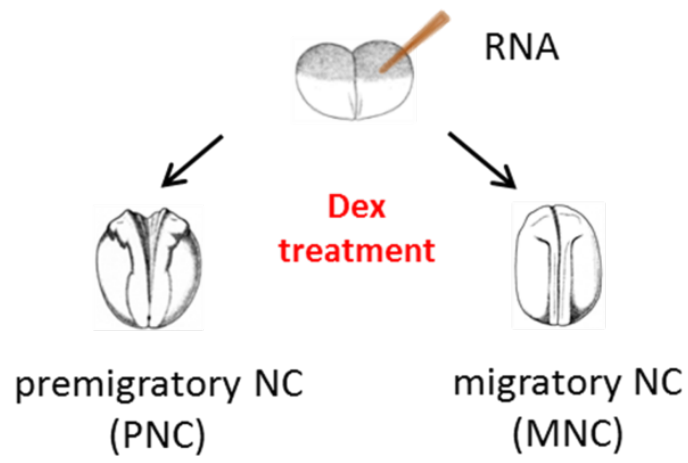

Figure.4.3.1 Experimental scheme. mRNA coding for a particular GR construct was injected into one blastomer of 2-cell stage embryos. The respective fusion-protein was activated by Dexamethasone treatment at premigratory or migratory $\mathrm{NC}$ stages. EtOH served as a solvent control. The given scheme was apply in each experiment using GR-constructs. 
A

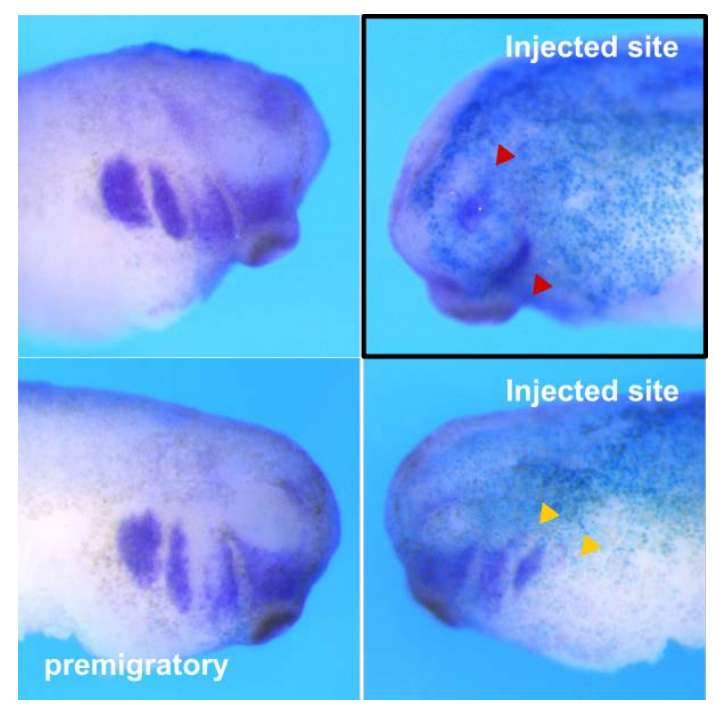

B

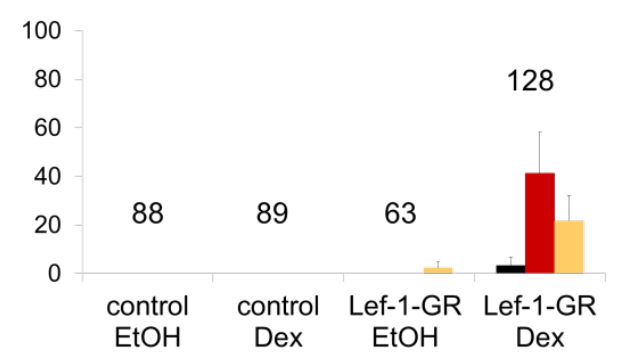

C

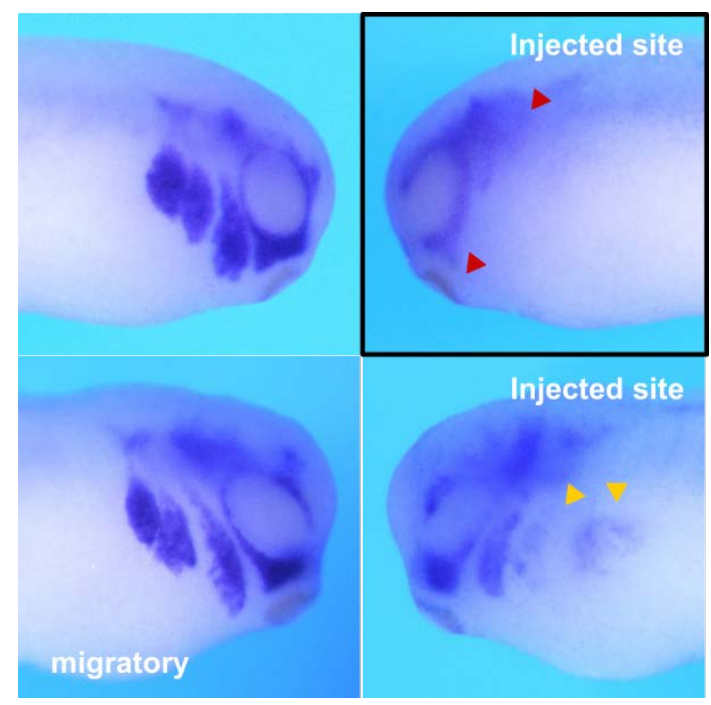

D

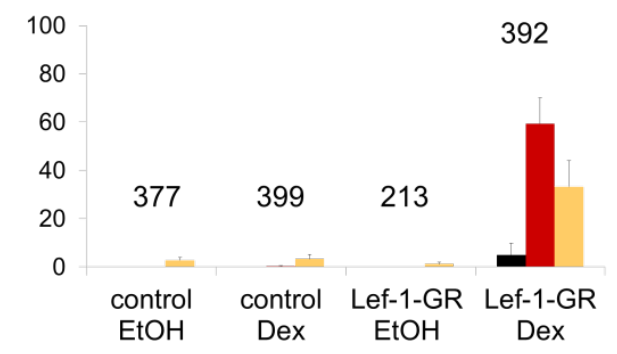

no NC

inhibited miogration

reduced branches

merged branches

Figure 4.3.1. Activation of canonical Wnt signaling at premigratory and migratory NC cell stages by Lef1GR overexpression affects NC migration A Wild type Xenopus embryos were injected with 50 pg of Lef-1-GR in 1 blastomer at the 2-cell stage embryos. Panel shows the different phenotypes observed after Lef1-GR activation at premigratory stages. Phenotypes are grouped by increasing severity: embryo with inhibited NC migration and reduced Twist expression (upper panel), embryo with reduced NC branches (lower panel). Red arrows mark a weakly stained NC cluster in the premigratory position and the remaining mandibular branch. Yellow arrows mark reduced NC branches. The black frame indicates the most abundant phenotype. B Graph summarizing the percentage of phenotypes observed in 6 independent experiments. Standard errors of the mean and number of injected embryos are indicated for each column. $\mathbf{C}$ The panel shows the different phenotypes observed after Lef1-GR activation at migratory stages. Phenotypes are grouped as in the premigratory treatment. D The graph summarizes 9 independent experiments. Standard errors of the mean and number of injected embryos are indicated for each column. 
Consistent with these findings activation of Tcf3-VP16-GR at both premigratory and migratory stages, also caused defects already observed upon BIO treatment and Lef1-GR overexpression. Interestingly, overexpression of Tcf-VP16-GR caused mostly the 'reduced branches' phenotype both at premigratory and migratory stages (Fig.4.3.2). In summary, these findings show that activation of canonical Wnt signaling using either BIO-treatment or an inducible Lef1/Tcf3-GR construct results in similar NC migration defects.

A

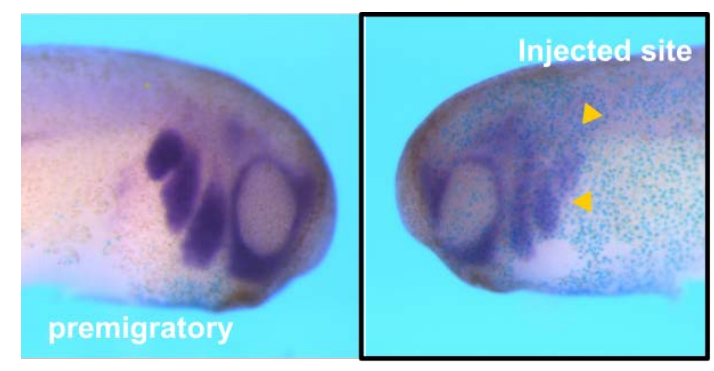

B

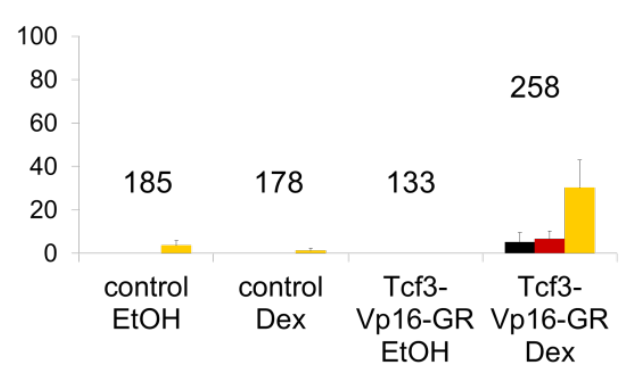

C

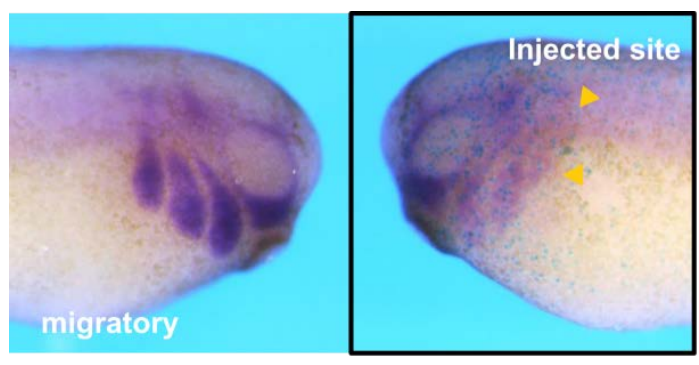

D

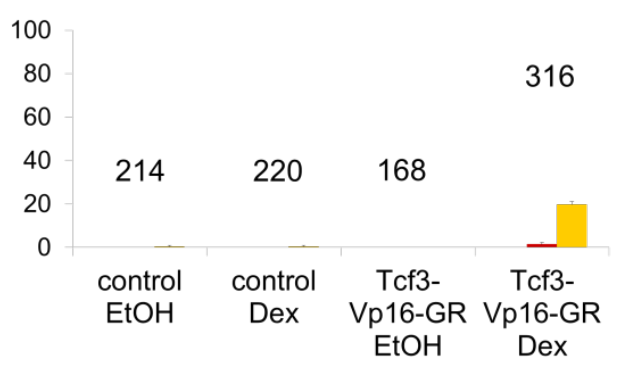

no NC

inhibited miogration

reduced branches

merged branches

Figure 4.3.2. Activation of canonical Wnt signaling at premigratory and migratory NC cell stages by Tcf3VP-16-GR overexpression affects NC migration A Wild type Xenopus embryos were injected with 50 pg of Tcf3-VP16-GR at 1 blastomer at 2-cell stage embryos. Panel shows embryo with reduced NC branches (yellow arrows), which is the predominant phenotype observed after Tcf3-VP16-GR activation at premigratory stages. B Graph summarizing 5 independent experiments. Standard errors of the mean and number of injected embryos are indicated for each column C Panel shows embryo with reduced NC branches (yellow arrows) representing the predominant phenotype observed after Tcf3-VP16-GR activation at migratory stages. Injected site is shown on the right. D The graph summarizes 5 independent experiments. Standard errors of the mean and number of injected embryos are indicated for each column.

\subsection{Activation of canonical Wnt signaling also causes defects in migration of Sox10-positive NC cells}

Twist is a bona fide NC marker, expressed in all areas of the cranial NC at premigratory and migratory stages and therefore well suited for investigating NC 
migration. As, not all cranial NC cells express Twist, the analysis of migration behavior upon activation of canonical Wnt signaling was additionally extended to the other cranial NC population, namely the Sox10-positive NC cells. Sox10 is initially expressed in NC cells from all axial levels, but at tailbud stages downregulated in the cranial NC (Aoki et al., 2003). Therefore, we focused on treatments at premigratory stages and analyzed the migration of Sox10-positive NC cells by in situ hybridization at early tailbud stages (22-24). As expected, treatment with BIO affected the migration of Sox10-positive cells (Fig.4.4.1A, B). Embryos showed either an inhibition of migration (Fig. 4.4.1A, panel III, red arrowheads; Fig. 4.4.1B, red bars) or merged NC branches, where the Sox10-expressing otic placode, could not be distinguished from the NC cells (Fig. 4.4.1A, panel II, Fig. 4.4.1B green bars). On the contrary to Twist-positive NC cell, a reduction of Sox10 was not observed.
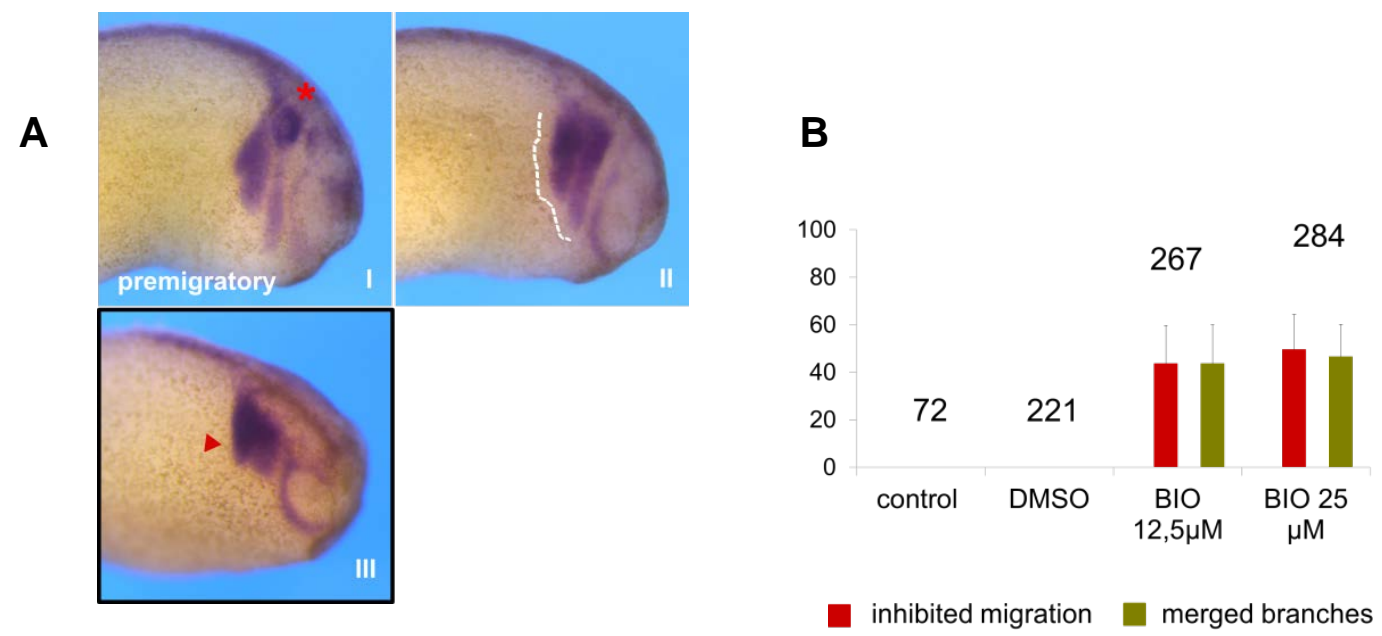

Figure 4.4.1. Activation of canonical Wnt signaling by BIO treatment affects the migration of Sox10positive NC cells. Wild type Xenopus embryos were treated with BIO at premigratory NC stages and DMSO treatment served as a solvent control. A The panel shows the different phenotypes observed after BIO treatment at premigratory stages. Phenotypes are grouped by increasing severity: unaffected embryo (I), embryo with merged NC branches (II), embryo with inhibited NC migration (III). Red arrows mark cells with inhibited migration and the remaining mandibular branch; merged NC cells are indicated by a dashed line. Black frame marks the most abundant phenotype. B Graph summarizing 4 independent experiments. Standard errors of the mean and number of treated embryos are indicated for each column.

Similar effects were observed, if canonical Wnt signaling was activated using the Lef1-GR construct. In this case, similarly to BIO treatment, Sox10-positive NC cells remained in a premigratory position (Fig. 4.4.2C, upper panel, red arrowhead; Fig. 4.4.2D, red bar) or NC cells migrated to some extent, but the streams appeared to be merged (Fig. 4.4.2C, lower panel; Fig. 4.4.2D, green bar). This latter, less severe, 
phenotype was also observed if canonical Wnt signaling was activated using Tcf3VP16-GR, while inhibited migration was rarely observed (less than 2\%). Thus, activation of canonical Wnt signaling using BIO or inducible Lef/Tcf constructs also affects the migration of Sox10-positive NC cells.

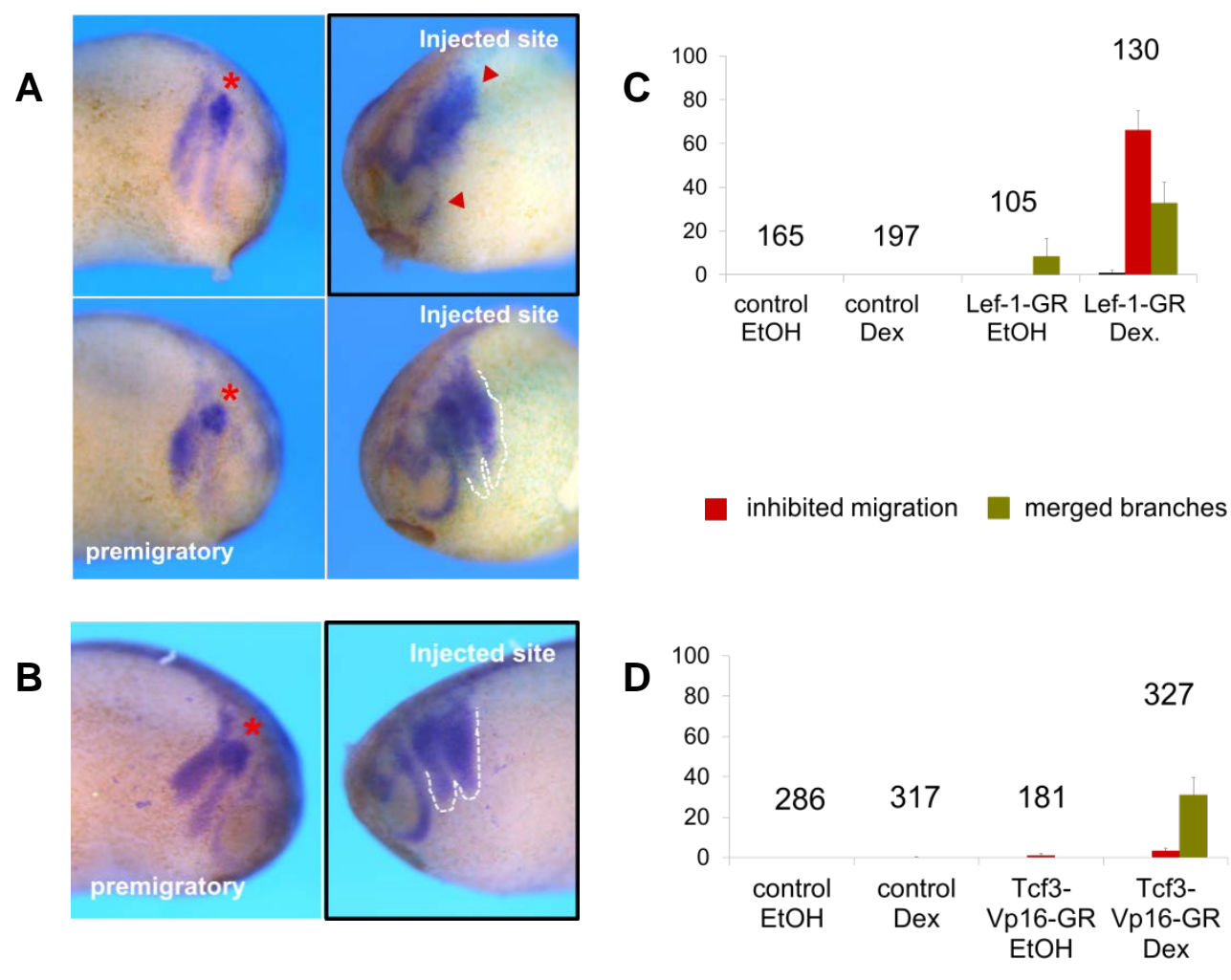

Figure 4.4.2. Activation of canonical Wnt signaling by overexpression of Lef1-GR and Tcf3-VP16-GR affects the migration of Sox10-positive NC cells. Wild type Xenopus embryos were injected with $50 \mathrm{pg}$ of Lef1-GR or Tcf3-VP16-GR in 1 blastomer at the 2-cell stage embryo. A The panel shows the different phenotypes observed after Lef1-GR activation at premigratory stages. Phenotypes are grouped by increasing severity: embryo with a strong inhibition of NC migration (upper panel), embryo with merged NC branches (lower panel). Red arrows mark NC cluster in the premigratory position and the remaining mandibular branch; merged NC cells are indicated by a dashed line. Black frame marks the most abundant phenotype. B Graph summarizing 5 independent experiments. Standard errors of the mean and number of injected embryos are indicated for each column. C The panel shows an embryo with merged NC branches, which is the predominant phenotype observed after Tcf3-VP16-GR activation at premigratory stages. Black frame marks the most abundant phenotype. D Graph summarizing 6 independent experiments. Standard errors of the mean and number of injected embryos are indicated for each column. 


\subsection{Inhibition of canonical Wnt signaling by IWR-1 treatment causes the same defects in migration of NC cells as BIO treatment}

To determine if inhibition of canonical Wnt signaling also has an influence on cranial NC cell migration, embryos were treated with IWR-1, which inhibits canonical Wnt signaling by stabilizing the destruction complex (Chen et al., 2009; Myers et al., 2014). Treatment of embryos with IWR-1 at premigratory stages led mostly to a fusion of the posterior Twist-positive NC branches corresponding to the less severe 'merged branches' phenotype already seen for BIO-treated embryos (Fig. 4.5A,B). The most posterior branchial NC streams did not separate properly and their migration was inhibited. IWR-1 treatment at migratory NC cell stages caused the same defects. Other defects like; 'inhibited migration' or 'reduced branches', which were caused by BIO-treatment, were only rarely observed.

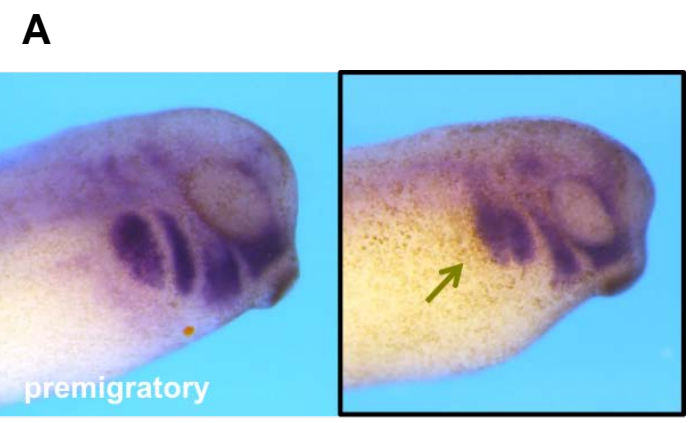

B

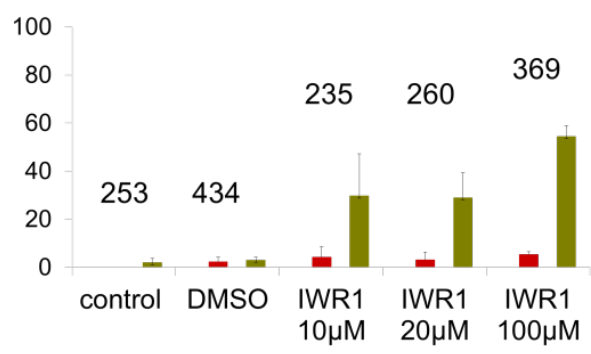

C

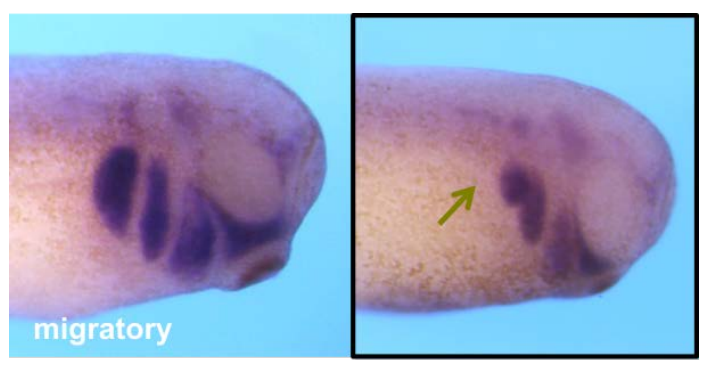

D

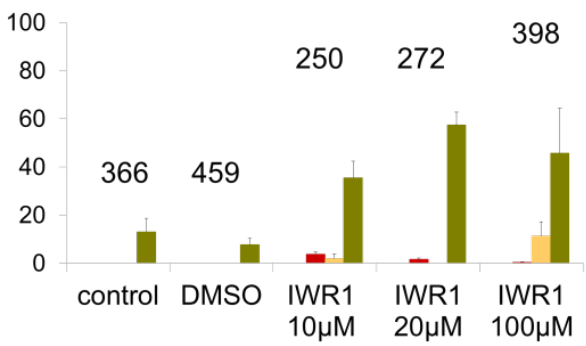

no NC

inhibited miogration

reduced branches

merged branches

Figure 4.5. Inhibition of canonical Wnt signaling using IWR1 at premigratory and migratory NC cell stages affects NC migration. Wild type Xenopus embryos were treated with IWR1 at NC premigratory and migratory stages or with DMSO as a solvent control. A The panel shows an embryo with merged NC branches (right panel, black frame, green arrow), the predominant phenotype observed after IWR1 treatment at premigratory stages. An untreated control embryo is shown on the left. B Graph summarizing the percentage of phenotypes of 4 independent experiments. Standard errors of the mean and number of treated embryos are indicated for each column. C The panel shows an embryo with merged NC branches (right panel, green arrow) after IWR1 treatment at migratory stages. A control embryo is shown on the left. D The graph summarizes 3 independent experiments. Standard errors of the mean and number of treated embryos are indicated for each column. 


\subsection{Functionality of Lef1/Tcf3 constructs in ventralization axis assay}

Since IWR-1 also works at the level of the cytoplasm and could therefore modulate wider spectra of intercellular processes we asked if direct inhibition of Lef1/Tcf3 signaling also affects NC migration. As for the activation, the functionality of the respective Tcf/Lef glucocorticoid-receptor (GR)-fusion constructs in inhibiting canonical Wnt signaling was assessed by analyzing their ability to induce ventralization of Xenopus embryos (Supplement Fig.6.2) (all tested by Dr. Juliane Melchert PhD Thesis:- Expression screen for Wnt signaling-like phenotypes identifies Fam132b as a novel inhibitor of BMP signaling in Xenopus). Again, form the panel of potential inhibitors only EnR-Lef-1-GR (Lyons et al., 2009) and Tcf3 $\Delta$ C-GR (Pukrop et al., 2001) were able to ventralize Xenopus embryos (Fig.4.6).

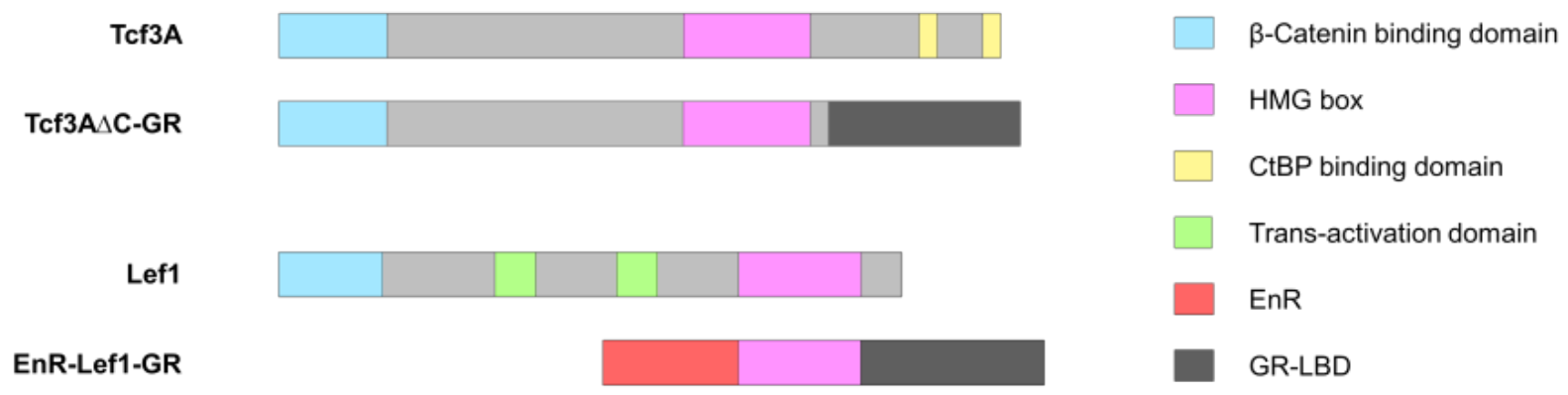

Figure 4.6. EnR-Lef-1-GR and Tcf3 $\triangle$ C-GR inhibit canonical Wnt signaling in early Xenopus embryos as indicated by secondary axis induction. A Schematic representation of wild type and modified GR-fusion constructs of Tcf3 and Lef1 used for inhibition of canonical Wnt signaling. Tcf3 $\triangle \mathrm{C}-\mathrm{GR}$ is a construct lacking CtBP binding domain (generated by Dr. Juliane Melchert), while EnR-Lef1-GR instead of ß-catenin binding domain and trans-activation domain has Drospohila engrailed repressor domain (Lyons et al., 2009)

\subsection{Inhibition of Tcf3 signaling causes the same defects in migration of NC cells as Lef1/Tcf-activation}

Interestingly, overexpression of EnR-Lef-1-GR caused no defects in the migration of Twist-positive NC cells either at premigratory or migratory stages (Supplement Fig.6.3). On the contrary, overexpression of Tcf3 $\Delta C-G R$, which showed high ventralization potency, at both premigratory and migratory stages resulted mainly in occurence of a less severe 'reduced branches' phenotype observed already upon 
overexpression of Lef1/Tcf3 activators (Fig. 4.7A,B lower panel, yellow arrowheads; Fig. 4.7C,D, yellow bars). In addition, Tcf3 $\Delta \mathrm{C}-\mathrm{GR}$ also caused the more severe 'inhibited migration' phenotype (Fig. 4.7A,B, upper panel, red arrowheads; Fig. 4.7C,D, red bar).

A

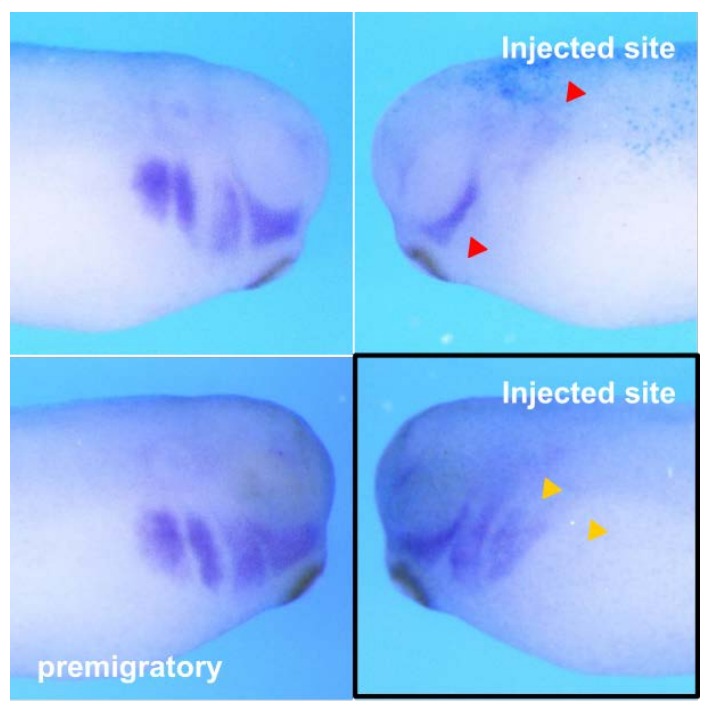

B

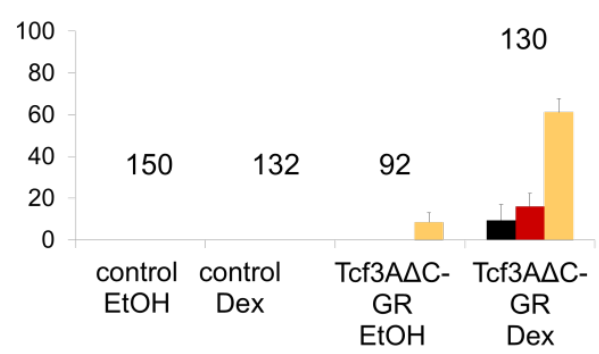

no NC
C

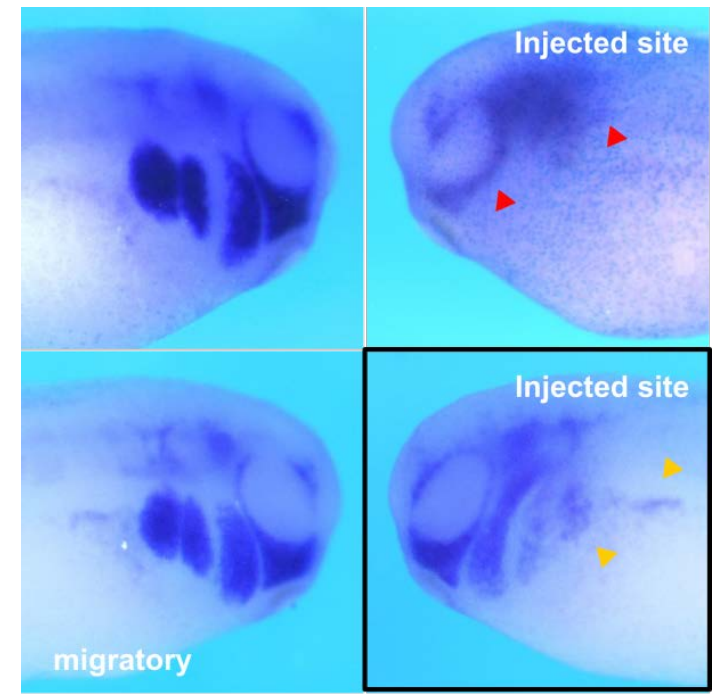

D

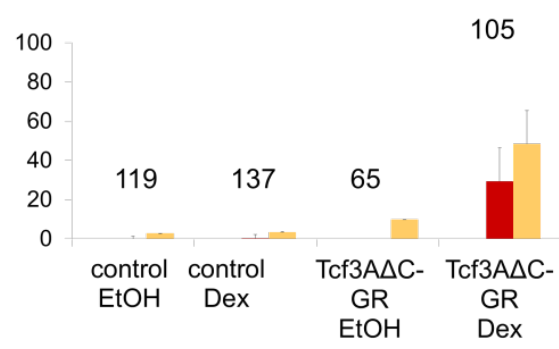

Figure 4.7. Inhibition of canonical Wnt signaling at premigratory and migratory NC cell stages by Tcf $\triangle \mathrm{C}$ 16-GR overexpression affects NC migration A Wild type Xenopus embryos were injected with Tcf3 $\triangle C$-GR RNA in one blastomere at the 2-cell stage. The panel shows the different phenotypes observed after Tcf3 $\triangle \mathrm{C}$-GR activation at premigratory stages. Phenotypes are grouped by increasing severity: embryo with inhibited NC migration and reduced Twist expression (upper panel), embryo with reduced NC branches (lower panel). Red arrows mark a weakly stained NC cluster in the premigratory position and the remaining mandibular branch. Yellow arrows mark reduced NC branches. Black frame indicates the most abundant phenotype. B Graph summarizing the percentage of phenotypes in 9 independent experiments. Standard errors of the mean and number of injected embryos are indicated for each column. $\mathbf{C}$ The panel shows the different phenotypes observed after Tcf3 $\triangle \mathrm{C}$-GR activation at migratory stages. The same phenotypes as for the premigratory Dexamethasone treatment are observed. D Graph summarizing 3 independent experiments. Standard errors of the mean and number of injected embryos are indicated for each column. 


\subsection{Inhibition of canonical Wnt signaling also causes defects in migration of Sox10-positive NC cells}

In addition we analyzed if inhibition of canonical Wnt signaling affects the migration of Sox10-expressing NC cells. The defects observed after treatment with IWR1 were comparable to the ones observed for the Twist-positive cells, but only a low percentage of embryos were affected (Fig. 4.8.1A,B). Sox10-positive cells remained in a premigratory position and only the first mandibular branch showed some migrating NC cells. As in the case of the Twist-positive NC cells, inhibition of canonical Wnt signaling by overexpressing the Tcf3 $\triangle \mathrm{C}$-GR construct strongly inhibited the migration of the Sox10-expressing NC cells. They clustered in a premigratory position and only cells of the mandibular arch migrated ('inhibited migration' phenotype) (Fig. 4.8.2C, upper panel; Fig. 4.8.2D). In addition a less severe phenotype was observed where the branchial NC streams merged and the Sox10-staining of the otic placode could not be distinguished from the NC staining (Fig. 4.8.2C, lower panel; Fig. 4.8.2D). Thus, these data are consistent with our previous findings on the migration of Twist expressing cells and show that overactivation or inhibition of canonical Wnt signaling affect NC migration.

A

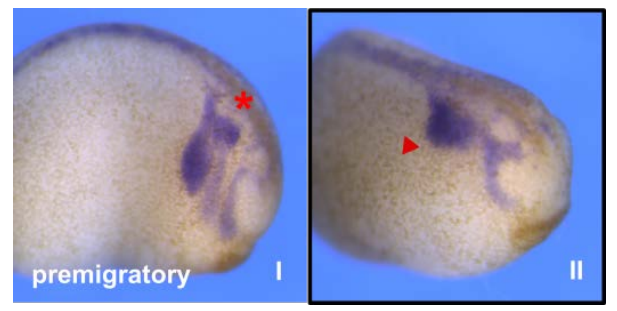

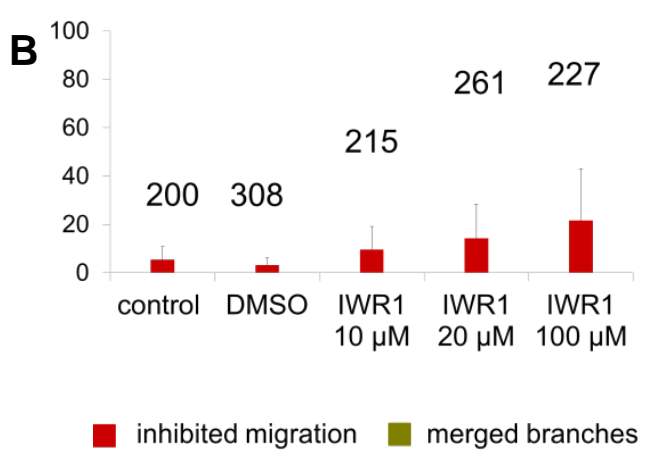

Figure 4.8.1. Inhibition of canonical Wnt signaling by IWR-1 treatment affects the migration of Sox10positive NC cells. Wild type Xenopus embryos were treated with IWR-1 at premigratory NC stages and DMSO treatment served as a solvent control. A Panel shows an unaffected embryo (I) and the 'inhibited migration' phenotype (II) observed after IWR1 treatment at premigratory stages. Black frame marks the only observed phenotype B Graph summarizes 4 independent experiments for $10 \mu \mathrm{M}$ and $20 \mu \mathrm{M}$ of IWR1 and 3 independent experiments for $100 \mu \mathrm{M}$ of IWR1. Standard errors of the mean and number of injected embryos are indicated for each column. 
A

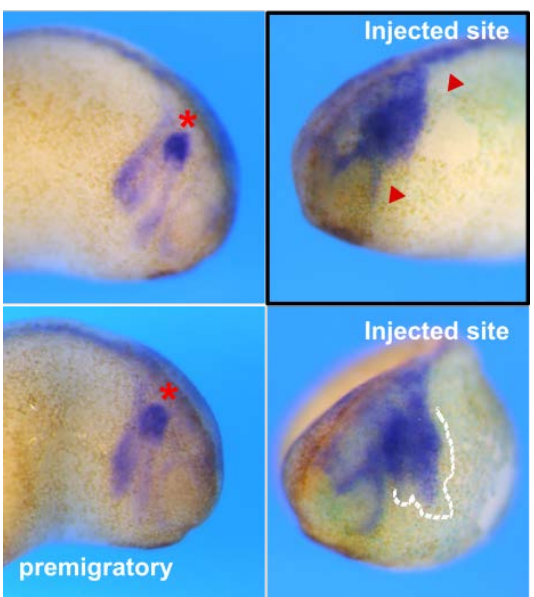

B

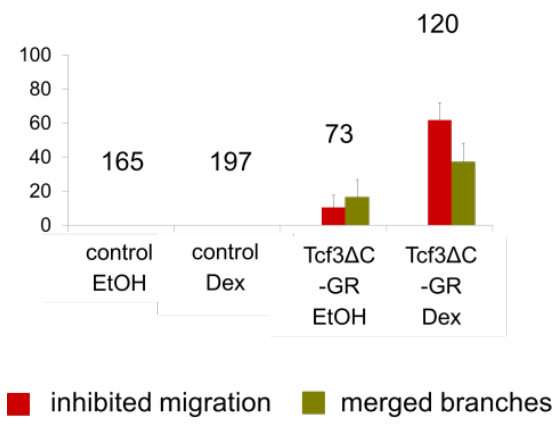

Figure 4.8.2. Inhibition of canonical Wnt signaling by overexpression of Tcf $3 \Delta \mathrm{c}-\mathrm{GR}$ affects the migration of Sox10-positive NC cells Wild type Xenopus embryos were injected with 50pg of Tcf3 $\Delta \mathrm{C}$-GR at 1 blastomer at 2-cell stage embryo. A The panel shows the different phenotypes observed after Tcf3 $\Delta \mathrm{C}$-GR activation at premigratory stages. Phenotypes are grouped by increasing severity: embryo with a strong inhibition of NC migration (upper panel), embryo with merged NC branches (lower panel). Red arrows mark NC cluster in the premigratory position and the remaining mandibular branch; merged NC cells are indicated by a dashed line. Black frame marks the most abundant phenotype. B Graph summarizing the percentage of phenotypes in 5 independent experiments. Standard errors of the mean and number of injected embryos are indicated for each column.

\subsection{Activation of canonical Wnt signaling by BIO affects in vitro NC migration}

To investigate the role of canonical Wnt signaling in the orchestration of NC migration on the cellular level, we used life-cell imaging of explanted NC cells. For this type of experiment, NC cells were dissected from embryos at premigratory NC cell stages and cultured in medium containing either Dexamethasone or BIO/IWR1 for 2 to 3 hours before imaging. Controls were cultured in the respective solvent controls. For each experimental condition, being it treatment with a chemical modulator or overexpression of a GR-inducible construct, a control from the same embryonic batch was used. Cell movements were monitored using spinning disk time-lapse imaging. As expected from the results in whole embryos, activation of canonical Wnt signaling using BIO-treatment inhibited NC migration (Mov.1 Supplement, Fig.4.9). Border cells 
formed proper lamellipodia as long as they remained in the NC cluster, however, these were lost upon detachment and cells were not able to move any further. In contrast control cells left the NC clusters efficiently and performed single cell migration.

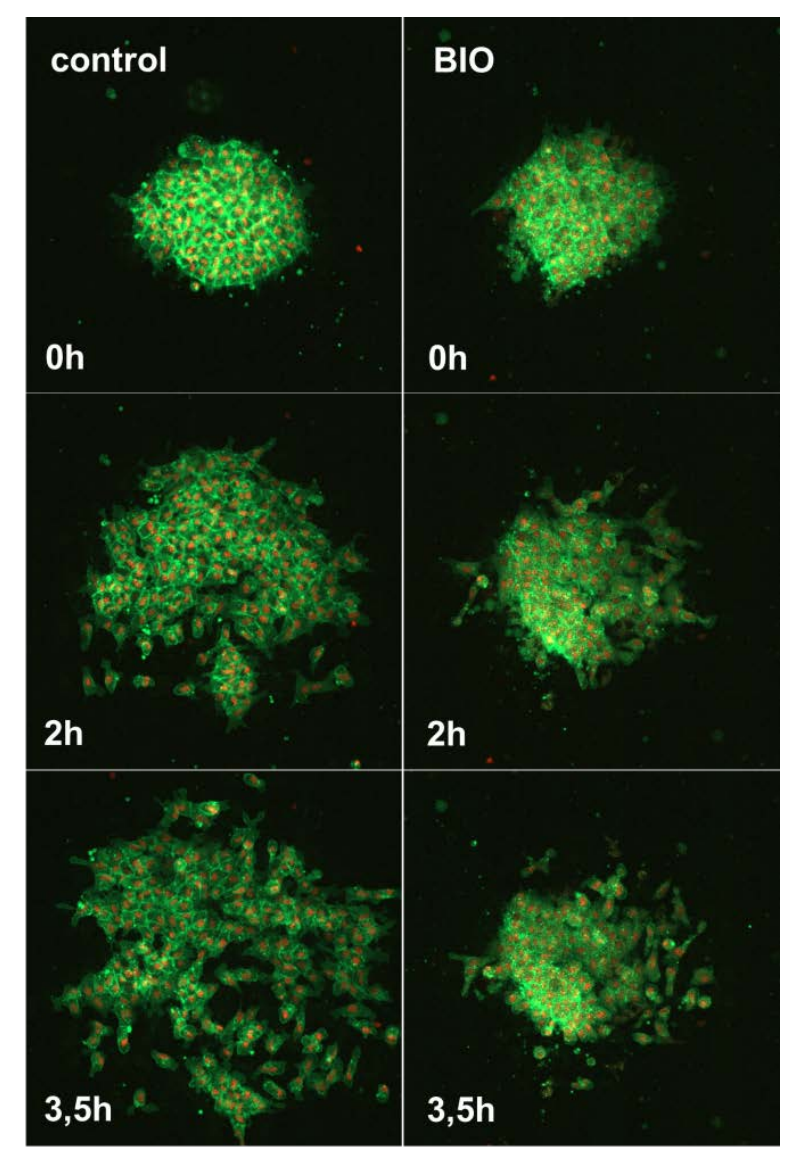

Figure 4.9. BIO treatment inhibits migration of explanted NC cells. Time-lapse images of control (left panels) and BIO-treated NC cells (right panels) at the start of imaging ( 0 hours) and after 2 and 3.5 hours. Cells were treated with $1 \mu \mathrm{M}$ BIO and DMSO as a control. Control NC cells leave the cluster and perform single cell migration. BIO treated NC cells show inhibited migration. Upon detachment from the cluster cell protrusions are retracted.

\subsection{Activation of canonical Wnt signaling by Lef1-GR affects in vitro NC migration}

Similary to BIO treatment, activation of canonical Wnt signaling using Lef1-GR overexpression also inhibited NC migration (Mov.2 Supplement, Fig.4.10.1). This effect was even more severe than upon BIO-treatment and Lef1-GR-overexpressing cells were unable to leave the NC cluster. Interestingly, the ability of the Lef1-GR- 
overexpressing NC cells to form lamellipodia was not affected. Conversely, control $\mathrm{NC}$ cells from the same batch rapidly left the main cluster and spread efficiently.

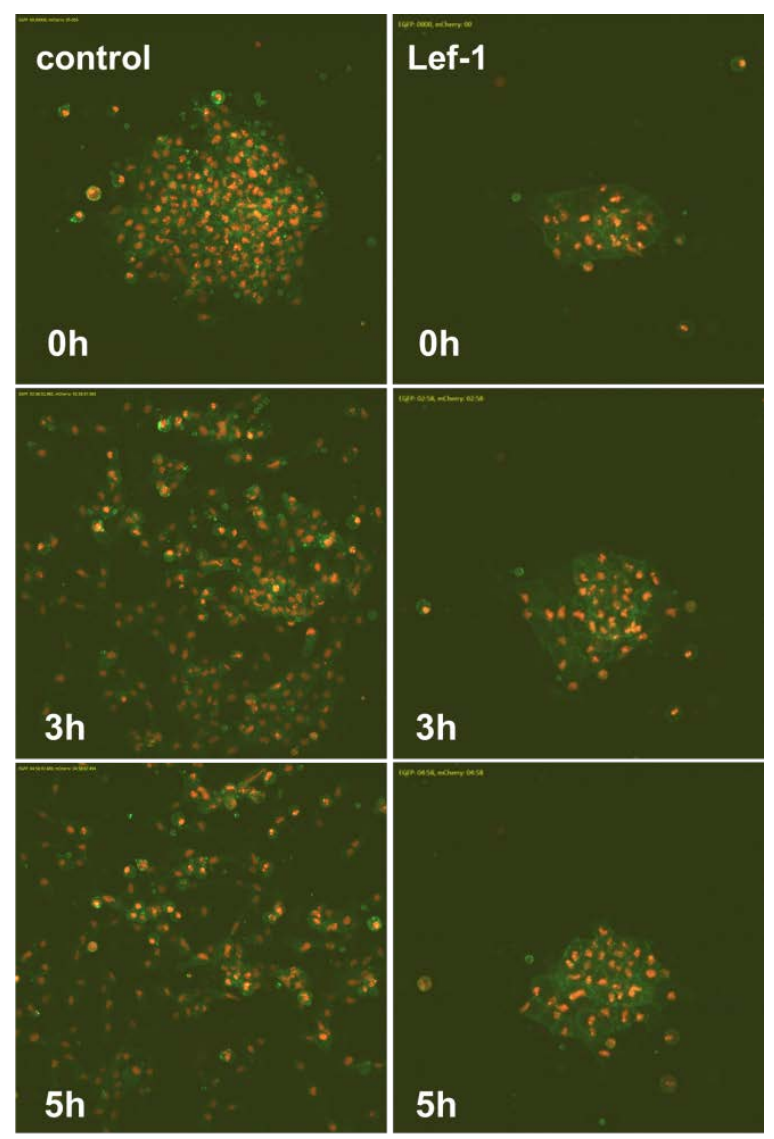

Figure 4.10.1. Lef-1-GR overexpression inhibits migration of explanted NC cells. Time-lapse images of control (left panels) and Lef1-GR-overexpressing NC cells (right panels) at the start of imaging (0 hours) and after 3 and 5 hours. Control NC cells leave the cluster and perform single cell migration. NC cells overexpressing Lef1-GR are not able to leave the cluster. Formation of cell protrusions is normal.

Migration of NC cells overexpressing Lef-1-GR was additionally characterized by cell classification (center, border, outsider cells; Fig.4.10.2.A), determination of mean cluster size and mean square displacement. These parameters were employed here to describe the cell migration behavior of explanted NC cells quantitatively. The definition, as well as calculation of these parameters is introduced in the Materials and Methods section. In short, successful NC migration is accompanied by a fast reduction in mean cluster size and an increase in outsider cells at the expense of center and border cells. These parameters were used to analyze how activation of canonical Wnt signaling by Lef1-GR affects NC migration. In control NC cells the mean cluster size dropped rapidly and center cells were quickly replaced by border 
and outsider cells (Fig. 4.10.2B,C). In contrast in Lef1-GR overexpressing explants the mean cluster size remained high and no significant increase in outsider cells at the expense of center cells was observed (Fig. 4.10.2D,E). Moreover, there was even an increase in mean cluster size as the explant stretched as the cells were trying to leave, but still remained attached together (Fig. 4.10.2D).
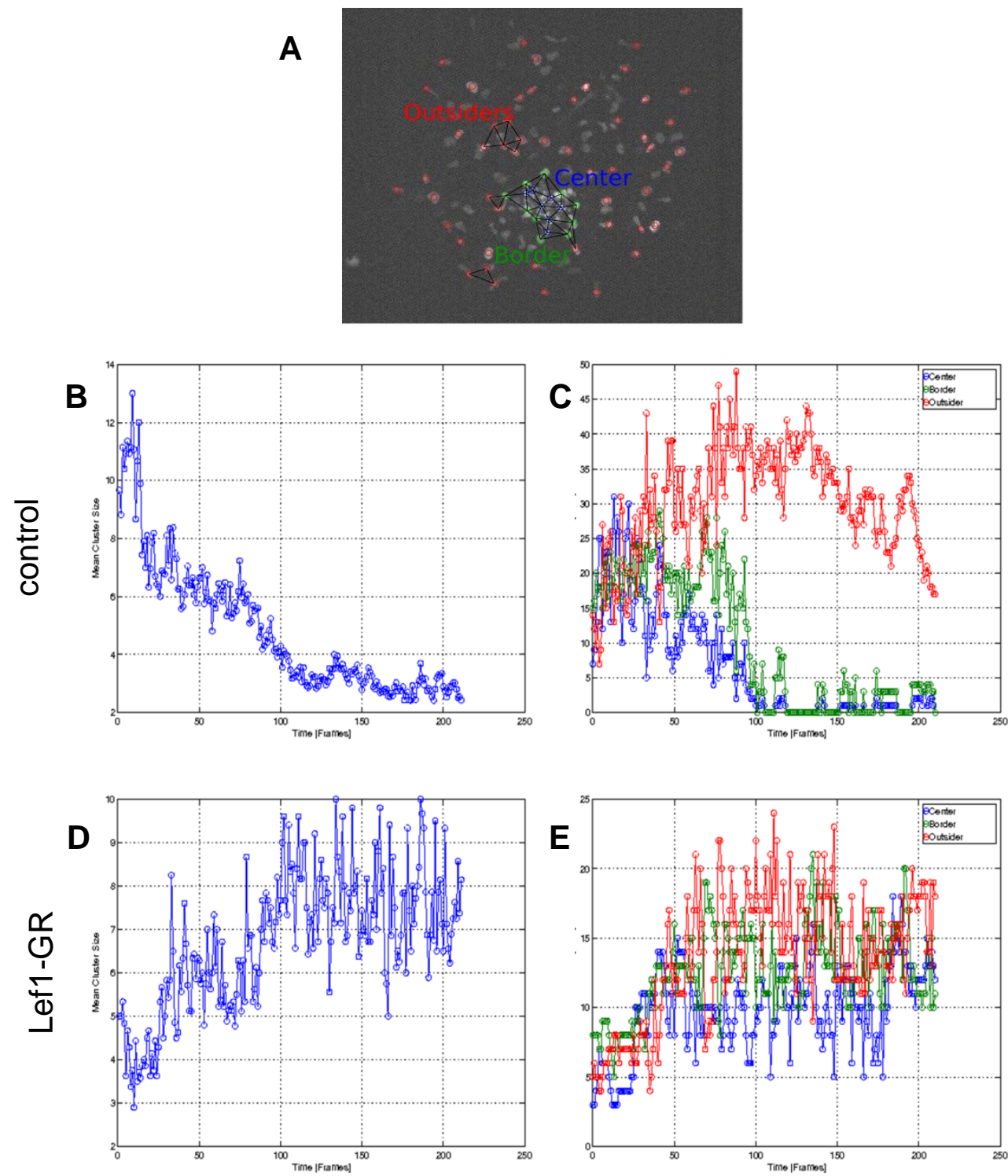

Figure 4.10.2. Data analysis of NC overexpressing Lef-1-GR and respective control A Classification of center, border and outsider cells. For classification criteria see Material and Method section. B Changes in mean cluster size of control NC explants over time. Time is given in "frames", which constitutes a time interval of 2.03 minutes. This is identical for all graphs shown in this figure. C Cell classification of a control NC explant. D Changes in mean cluster size of Lef1-GR-overexpressing NC explant. E Cell classification of a Lef-1-GRoverexpressing explant. Anlyzed was performed by Lutz Künneke. 


\subsection{Inhibition of canonical Wnt signaling by IWR1 affects in vitro NC migration}

By analogy to the whole embryos, treatment with IWR1 also exhibited less severe defects in migration of explanted NC cells. NC cells treated with IWR-1 formed proper lamellipodia, but shortly after leaving the cluster these were partially retracted, while controls showed normal migration behavior (Mov.3 Supplement, Fig.4.11). IWR1treated cells also continuously left the NC cluster, but due to their decreased mobility they remained in close proximity.

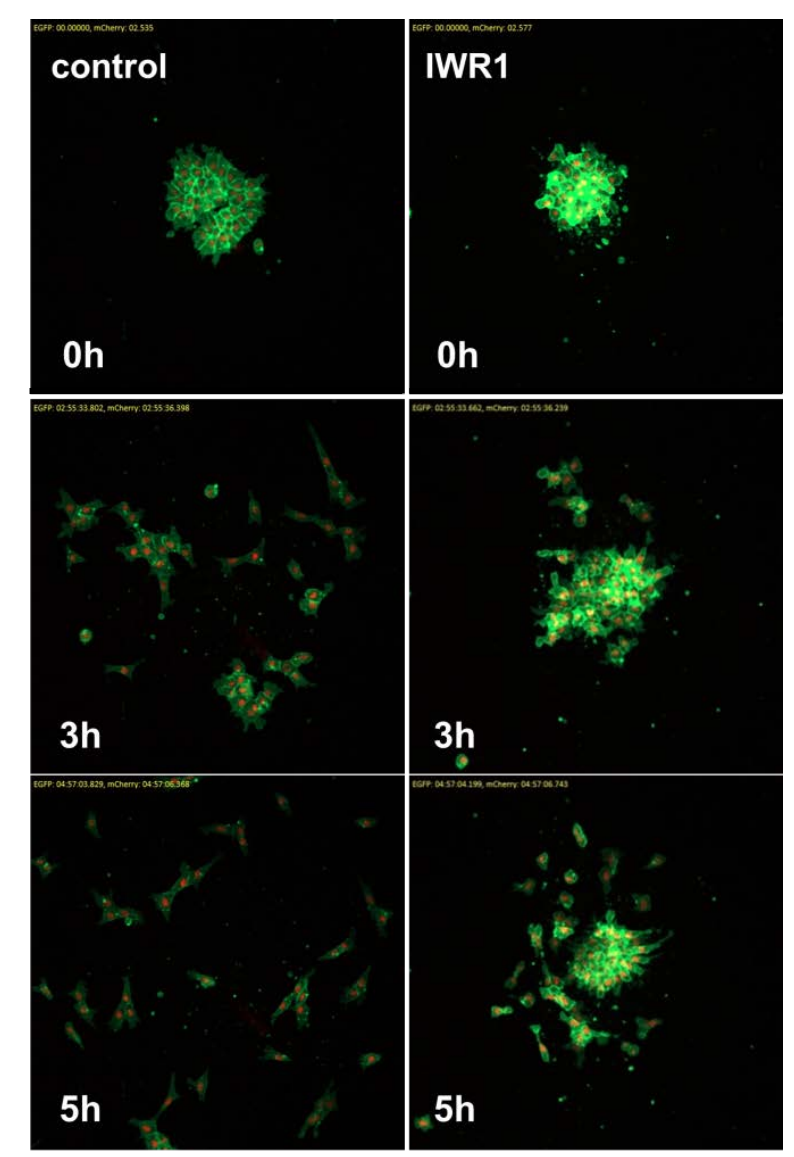

Figure 4.11. IWR1 treatment limits the motility of explanted NC cells. A Time-lapse images of control (left panels) and IWR1-treated NC cells (right panels) at the start of imaging (0 hours) and after 3 and 5 hours. Cells were treated with 2,5 $\mu \mathrm{M}$ IWR1 and DMSO as control. Control NC cells left the cluster and performed single cell migration. IWR1-treated NC cells show decreased motility. Upon detachment from the cluster cell protrusions are partially retracted. 


\subsection{Inhibition of canonical Wnt signaling by $\operatorname{Tcf} 3 \Delta \mathrm{C}$-GR affects in vitro NC migration}

As already seen in the whole embryos, in vitro overexpression of Tcf3 $\Delta \mathrm{C}$-GR also results in decreased severity of migration defects (Mov.4 Supplement, Fig.4.12.1). NC cells remained joined together while they migrated. Thereby, the whole cell cluster stretched, and only eventually cells were able to detach. Therefore, the mean cluster size remained at a steady state level and the appearance of outsider cells was severely delayed compared to the control (Fig. 4.12.2A-D). The rapid drop in the number of outsider cells in the control can be explained by migration of the big cell cluster out of the focus plane. Taken together, inhibition of canonical Wnt signaling using IWR or overexpression of Tcf3 $\Delta \mathrm{C}$-GR inhibited the motility of NC cells and their ability to perform single cell migration.

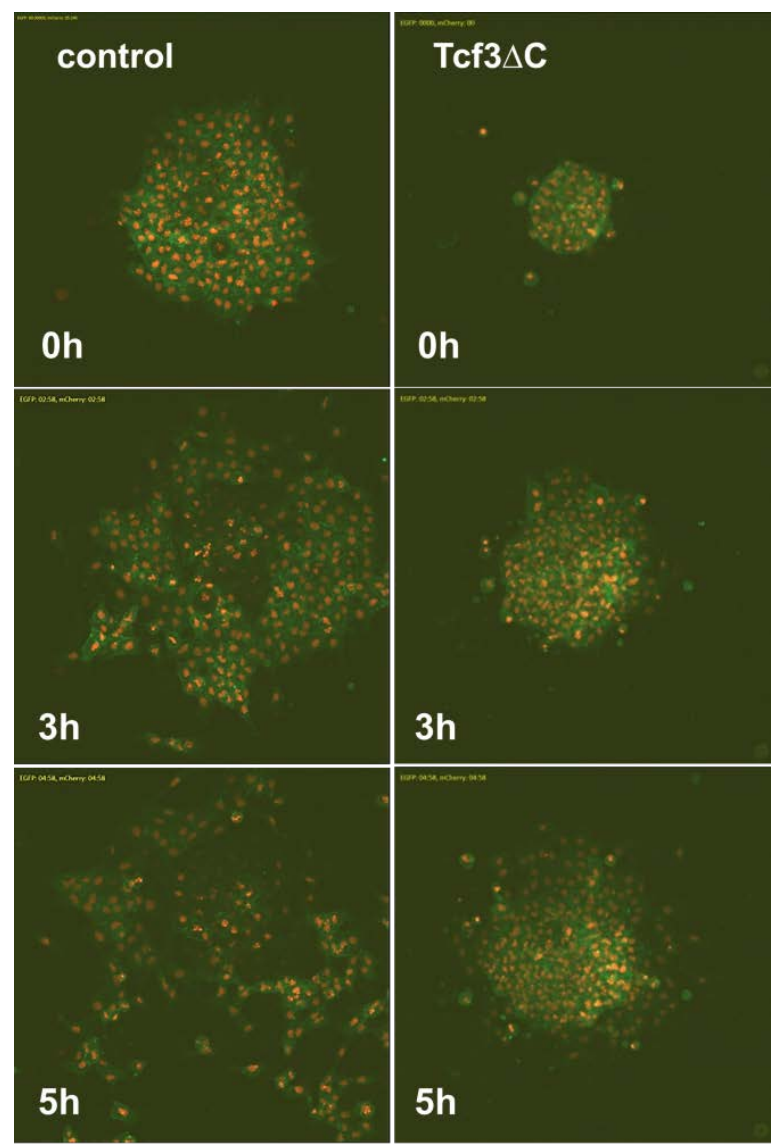

Figure 4.12.1. Tcf3 $\Delta$ C-GR overexpression limits motility of explanted NC cells. Time-lapse images of control (left panels) and Tcf3 $\Delta$ C-GR overexpressing NC cells (right panels) at the start of imaging (0 hours) and after 3 and 5 hours. Control NC cells leave the cluster and perform single cell migration. NC cells overexpressing Tcf3 $\Delta$ C-GR remain joined together and spread all over the fibronectin dish as one cluster. Gradually single NC cells are released from the collective. Formation of cell protrusions is normal. 

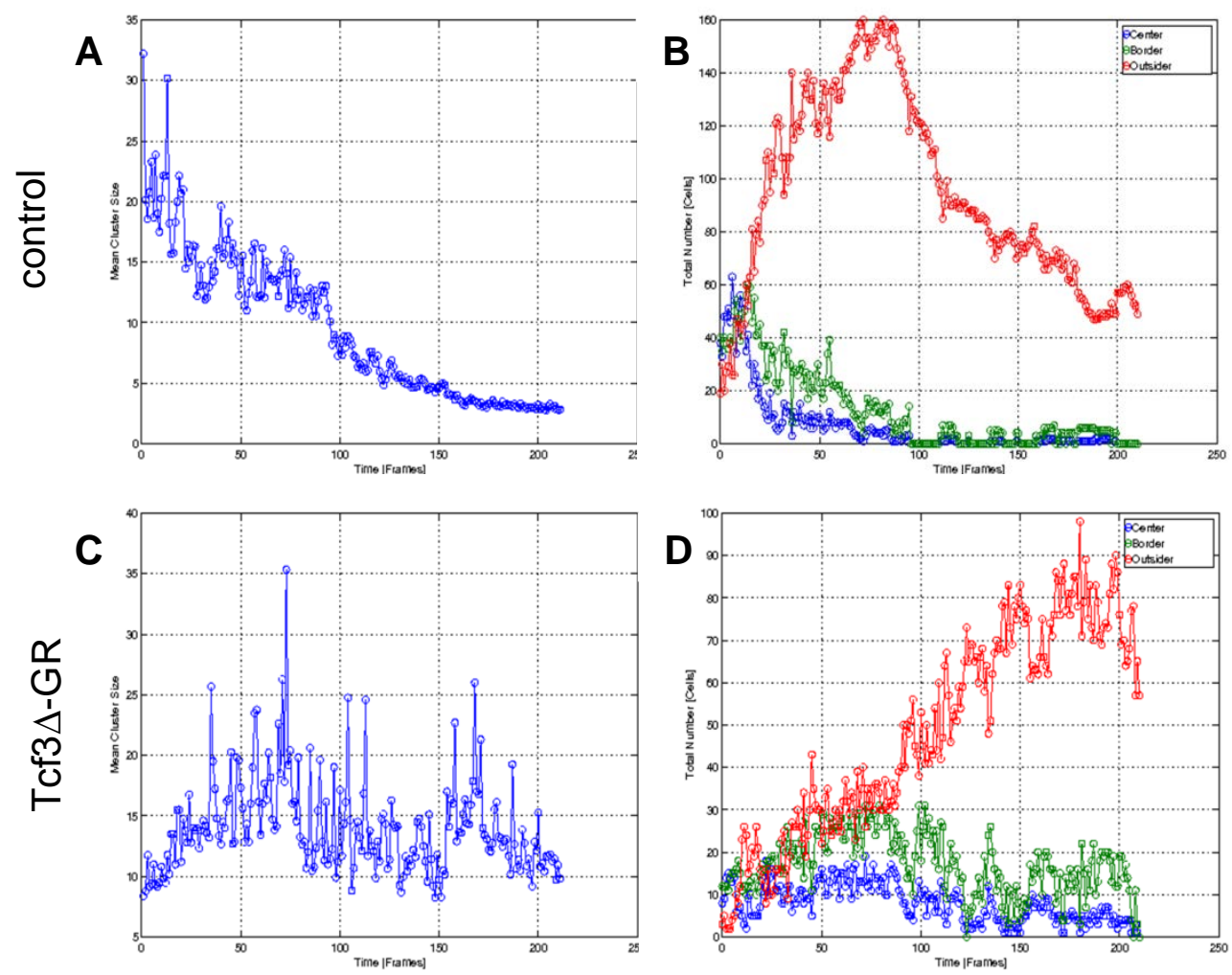

Figure 4.12.2. Data analysis of NC overexpressing Tcf3 $\Delta$ C-GR and respective control C Changes in mean cluster size of control NC cells over time. Time is given in "frames", which constitutes a time interval of 2.03 minutes. This is identical for all graphs shown in this figure. D Cell classification of a control NC explant. E Changes in mean cluster size of a Tcf3 $\Delta$ C-GR-overexpressing NC explant. F Cell classification of a Tcf3 $\Delta$ C-GRoverexpressing explant. Analysed was performed by Lutz Künneke.

\subsection{Comparison of Mean Square Displacement}

Determination of mean cluster size and cell classification were further confirmed by analyzing the mean square displacement (Fig.4.13) describing the dynamic properties of cell movement. Here cell movement during the starting phase of the experiment (first 2 hours, Fig. 4.13A) is compared to cell movement during a later time period of the experiment (4-6 hours, Fig. 4.13B), when cell movement is in general accelerated. Lef1-GR and Tcf3 $\Delta \mathrm{C}$-GR overexpression both result in a strong decrease of the mean square displacement in comparison to control NC cells. This effect is already apparent in the first 2 hours of the experiment (Fig. 4.13A) and is 
getting more severe during the course of the experiment (Fig. 4.13B). Control NC cells show in general a wider range of cell motility likely also depending on their position in explants or their position in respect to other migrating cells. In summary the in vitro migration data confirm the results obtained in whole embryos, but shows that cell mobility as well as the ability to leave the NC clusters is impaired.
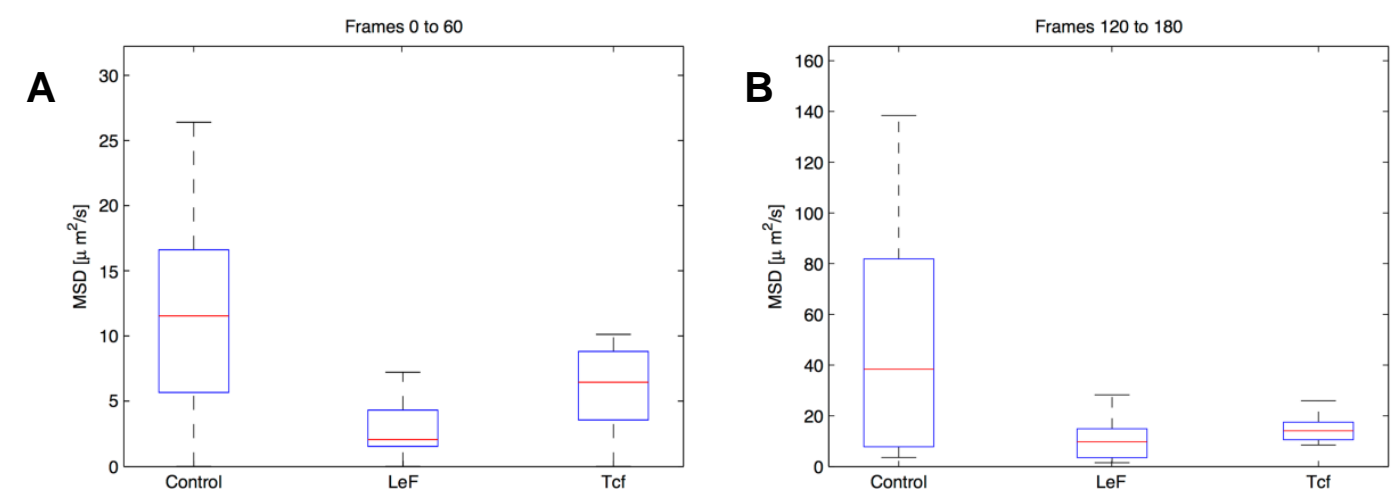

Figure 4.13. The mean square displacement confirms that modulation of canonical Wnt signaling inhibits in vitro NC migration. Comparison of the mean square displacement of control cells and NC cells overexpressing Lef1-GR or $\operatorname{Tcf} 3 \Delta \mathrm{C}$ respectively. The mean square displacement is given for cell movement during the first 2 hours of the experiment $\mathbf{A}$ compared to cell movements during a later time period in the course of the experiment (between 4 and 6 hours) B. Analysed was performed by Lutz Künneke.

\subsection{Endogenous nuclear ß-catenin levels decrease during NC migration}

As our data indicate that canonical Wnt signaling has to be tightly controlled to allow for NC migration, we asked if this is also reflected by the endogenous activity of canonical Wnt signaling during NC migration. Nuclear localization of $\beta$-catenin as readout for canonical Wnt activity was analyzed at different time points of NC migration (premigratory, early migratory, late migratory). Premigratory NC explants showed higher levels of nuclear $\beta$-catenin (Fig. 4.14.1/4.14.2) in comparison to migratory explants, where ß-catenin was mainly localized at the plasma membrane (Fig. 4.14.1/4.14.2). This indicates that during stages of NC migration nuclear ßcatenin levels decreased compared to the premigratory stage, consistent with the hypothesis that canonical Wnt activity has to be tuned down to allow for proper NC 
migration. In contrast, explants cultured in BIO-containing medium showed elevated levels of nuclear ß-catenin. While canonical Wnt activity - measured as nuclear localization of ß-catenin - decreased in control explants after 3 hours (Fig. 4.14.1/4.14.2), BIO-treated cells retained the high levels of nuclear ß-catenin as seen in premigratory NC cells (Fig. 4.14.1/4.14.2). This effect is even enhanced in explants treated for 7 hours with BIO (Fig. 4.14.1/4.14.2 ), where ß-catenin accumulation is visible in the cytoplasm as well as the nucleus. Considering that BIO treatment inhibits in vivo and in vitro NC migration (Fig. 4.1A-D, Fig. 4.9) ectopic activation of canonical Wnt signaling likely causes these defects. Thus, these data suggest that endogenous canonical Wnt activity has to be kept at a comparably low level to enable NC migration.

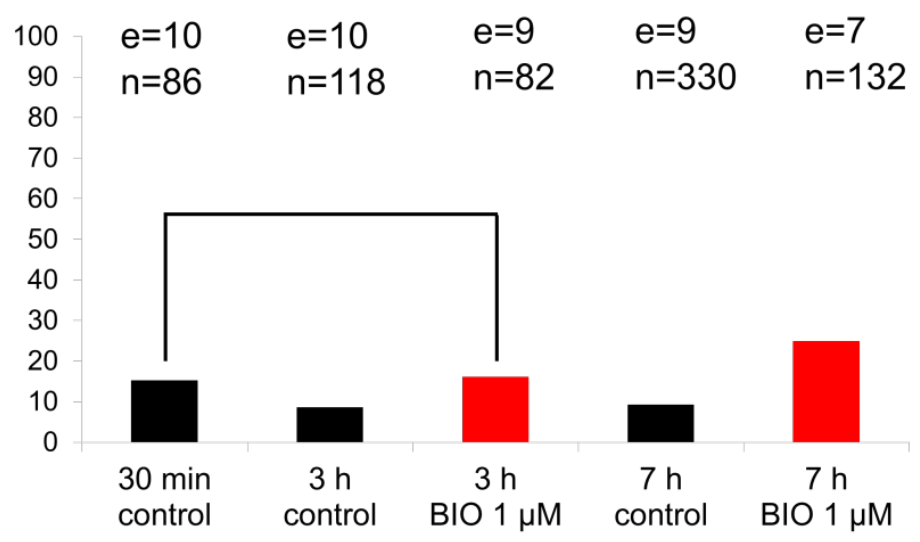

Figure 4.14.1 The levels of nuclear $\beta$-catenin change during stages of NC migration and are upregulated by BIO treatment. A A premigratory control NC explant (30 minutes after explantation) showing elevated levels of nuclear ß-catenin in comparison to control NC explants fixed 3 hours B or 7 hours $\mathbf{D}$ after explantation. NC explants treated with $1 \mu \mathrm{M}$ BIO for 3 hours $\mathbf{C}$ or 7 hours $\mathbf{E}$ showing translocation of ß-catenin into the nuclei and elevated levels of cytoplasmic ß-catenin (Fig.21, panel I and II). F Graph summarizing averaged fluorescent intensity expressed as Integrated Density of control and BIO-treated explants. The area of each nucleus was determined by DAPI staining (Fig.21) and the intensity of the ß-catenin fluorescent signal expressed as Integrated Density was measured using ImageJ. Integrated Densities of ß-catenin fluorescent signals from all explants of a particular treatment were averaged. One Way ANOVA and post hoc Games-Howell test were used for statistical analysis. Numbers of nuclei and explants analyzed are indicated for each column. BIO treatment leads to increased nuclear ß-catenin compared to controls. One Way ANOVA and post hoc Games-Howell test confirm that NC explants treated with $1 \mu \mathrm{M}$ BIO for 3 hours $\mathbf{C}$ show similar levels of nuclear ß-catenin compared to untreated NC explants fixed 30 min after explantation $\mathbf{A}$. 


\begin{tabular}{|c|c|c|c|c|}
\hline $30 \mathrm{~min}$ B-catenin & I & $30 \mathrm{~min}$ B-catenin & $\begin{array}{l}30 \text { min } \\
\text { B-catenin +DAPI }\end{array}$ & 30 min Twist \\
\hline $3 \mathrm{~h}$ control & I & $3 \mathbf{~ h}$ control B-catenin & $\begin{array}{l}3 \mathrm{~h} \text { control } \\
\text { B-catenin +DAPI }\end{array}$ & 3 h control Twist \\
\hline 3 h BIO & I & $3 \mathrm{~h}$ BIO B-catenin & $\begin{array}{l}3 \text { h BIO } \\
\text { B-catenin +DAPI }\end{array}$ & 3 h BIO Twist \\
\hline $7 \mathrm{~h}$ control & I & $7 \mathrm{~h}$ control B-catenin & $\begin{array}{l}7 \mathrm{~h} \text { control } \\
\text { B-catenin +DAPI }\end{array}$ & $\begin{array}{c}\text { 7h control-Twist } \\
0 \\
0\end{array}$ \\
\hline 7 h BIO & I & 7 h BIO B-catenin & $\begin{array}{l}7 \text { h BIO } \\
\text { B-catenin +DAPI }\end{array}$ & 7 h BIO Twist \\
\hline
\end{tabular}

Figure 4.14.2. The levels of nuclear $\beta$-catenin change during stages of $\mathrm{NC}$ migration and are upregulated by BIO treatment NC explants were dissected at premigratory stages and fixed at premigratory stages (30 minutes after explantation) or migratory stages (3 hours or 7 hours after explantation). B-catenin localization was determined by immunostaining (red, panel I and II). Panel I shows an optical section through the membrane layer of the explant, while panel II shows a section through a layer containing the nuclei. Panel III shows DAPI staining (blue) of the layer seen in panel II. Panel IV shows an immunostaining for Twist (green). 


\subsection{Modulation of non-canonical Wnt pathway}

In addition to modulation of canonical Wnt pathway, we also investigated the migration behavior of NC upon inhibition of non-canonical Wnt signaling. Here, we took advantage of three commercially available chemicals, including SP600125, Box5 and IWP-2. SP600125 inhibits phosphorylation of Jun N-terminal kinase (JNK) by competing with ATP (Bennett et al., 2001). JNK kinases are mostly linked with inflammation, proliferation and cell apoptosis, however, they have been also shown to play a role in cell migration. In Drosophila, JNK is required for dorsal closure (Riesgo-Escovar et al., 1996; Sluss et al., 1996), while in zebrafish and rat JNK1 is responsible for the rapid movement of keratocytes and bladder tumour epithelial cells respectively (Huang et al., 2003). Moreover, JNK phosphorylates paxillin, a focal adhesion adaptor, where its role in cell migration is well established. Mutation in serine residue phosphorylated by JNK causes formation of focal adhesions and limited cell movement indicating that phosphorylation of paxillin by JNK is crucial for labile adhesions facilitating rapid movement (Huang et al., 2003). Furthermore, activation of JNK has been also linked to the expression of metalloproteinase, which are also required during delamination of NC cells (Han et al., 2001). Since SP600125 has been shown to inhibit proliferation, angiogenesis, growth as well as migration of tumor cells (Bruce et al., 2004,) it might be an attractive tool to investigate inhibition of non-canonical Wnt signaling in migratory NC cells. Upon treatment of whole embryos with SP600125 we observed an inhibited migration of Twist-positive NC cells as well as a 'comb-shaped' NC streams, where all four stream where formed, but remained in close proximity with each other and seemed to be compact (Fig.4.15.1AB) Interestingly, a reduction of Twist expression was not observed. Treatment of explanted NC cells with SP1600125 caused an increase in cellsubstratum adhesiveness. NC cell formed proper protrusions in the direction of anticipated movement maintaining thereby directional polarity, but were unable to leave the NC cluster (Fig.4.15.2, Mov.5). Noteworthy, the whole NC cluster seemed to be highly immobilized and its movement was strongly limited. 
A

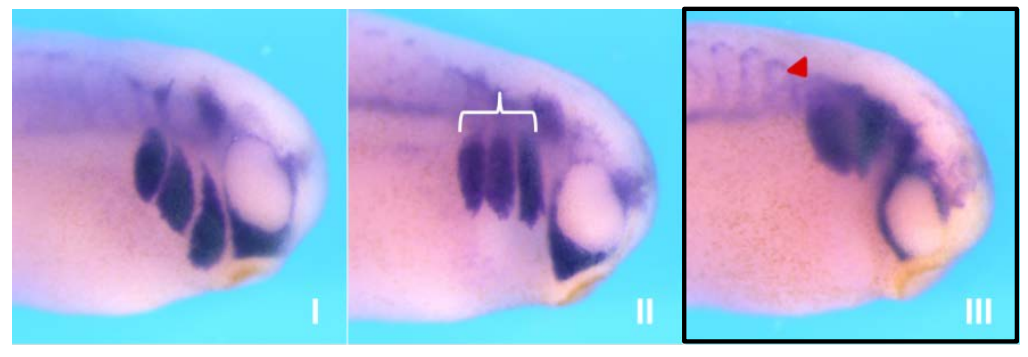

B

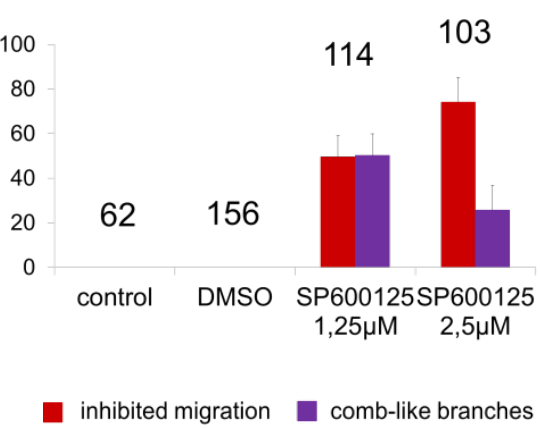

Figure.4.15.1. Inhibition of non-canonical Wnt signaling in whole embryos by treatment with the JNK inhibitor SP600125. Wild type Xenopus embryos were treated with increasing concentrations of SP600125 at NC premigratory stages or DMSO as a solvent control. A Panel shows an unaffected embryo (I), 'comb-like' phenotype with compacted NC branches (II) (white bracket) and 'inhibited migration' phenotype where clusters of NC remaining at the proximity of the neural tube (III) (red arrowhead). Black frame marks the most abundant phenotype B The graph summarizes three independent experiments. Standard errors of the means and number of treated embryos are indicated for each column.

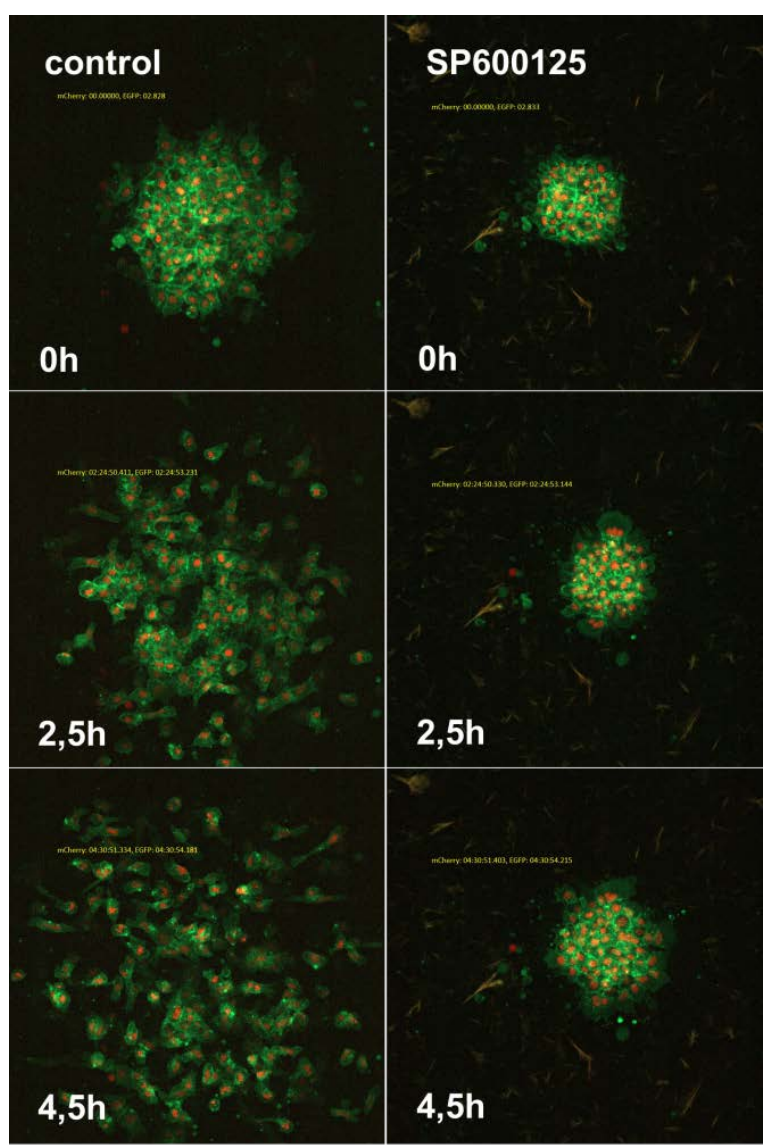

Figure.4.15.2. SP600125 treatment inhibits migration of explanted NC cells. A Time-lapse images of control (left panels) and SP600125-treated NC cells (right panels) at the start of imaging (0 hours) and after 2,5 and 4,5 hours. Cells were treated with $1 \mu \mathrm{M}$ SP600125 and DMSO as control. Control NC cells leave the cluster and perform single cell migration. SP600125-treated NC cells show decreased motility. NC cells do not detach from the cluster, but form normal cell protrusions. 
Another chemical modulator used in our studies is Box5, a Wnt5a-derived hexapeptide which has been shown to antagonize activities mediated by Wnt5a and Fz5 such as calcium response, cell invasion and migration in tumor cell lines (Jenei et al., 2009). Wnt5a can also bind Ror2 and this interaction is well established and classified as a separated branch of the Wnt pathway, namely Wnt5a/Ror2 pathway (Grenn et al., 2008, Minami et al., 2010). In Xenopus, activation of the Wnt5a/Ror2 pathway was shown to promote expression of paraxial protocedherin (XPAPC) which promotes convergent extension movements by acting through PI3K, Cdc-42, MKK7, JNK and C-jun (Schambony and Wedlich, 2007). What's more, expression of Ror2 in Xenopus cranial NC cells starts already at stage 15 (Hikasa et al., 2002), but so far expression of Wnt5a in NC cells has been reported in the early and mid-tadpole stages (Aaron Zorn Lab Xenbase Archive: XB-IMG-38521; Jung et al., 2011).

Treatment of the whole embryos with Box5 caused mostly formation of unstructured NC streams, however, the percentage of observed defects was very low (Fig.4.15.3AB). Defects resembling the 'inhibited migration' phenotype were not observed. Since Box5 is a hexapeptide it is likely that penetration through vitellin membrane and epidermis was impaired, therefore additional analysis would require higher concentrations, mechanical removal of the vitellin membrane as well as slight enzymatic digest of the epidermis using proteinase $\mathrm{K}$. As in the case of SP600125 treatment, reduction in Twist-positive NC cells through Box5 activity was either not observed or was mild. Treatment of explanted NC cells with Box5 phencopies migration behavior observed upon treatment with SP600125 (Fig.4.15.4. Mov.5). NC cells formed proper protrusion and exerted directional polarization, but showed limited ability in detaching from the NC cluster, which separated into smaller clusters migrating with decreased motility. In comparison to SP600125, Box treatment results in milder disturbances in NC migration both in vivo and in vitro experiments what could be credited to limited penetrance of Box5, but also the fact that Wnt5a seems to play a role in later migration of $\mathrm{NC}$ cells. Another possibility could be that Box5 structurally mimics other Wnt ligands. 
A

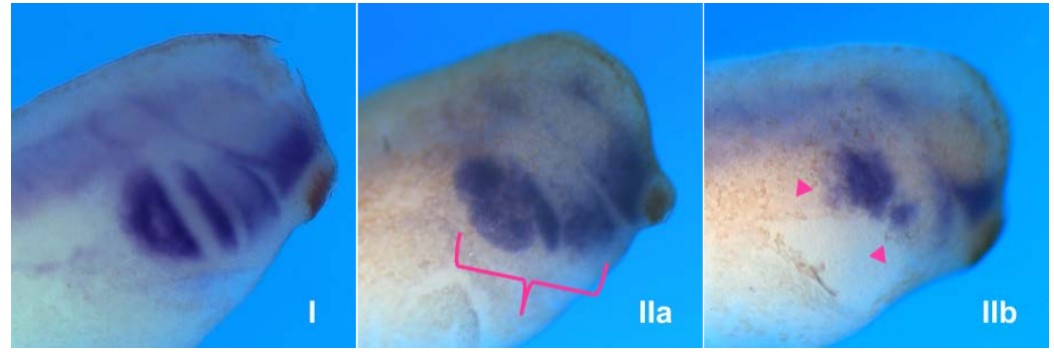

B

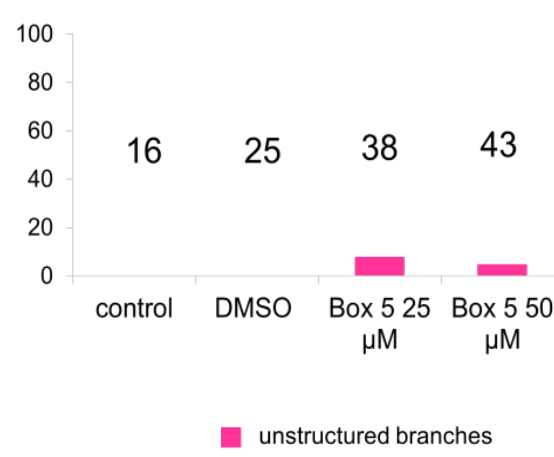

Figure.4.15.3. Inhibition of non-canonical Wnt signaling in whole embryos by treatment with Box5 mimicking Wnt5a. Wild type Xenopus embryos were treated with increasing concentrations of Box5 at NC premigratory stages or DMSO as a solvent control. A Panel shows an unaffected embryo (I), two variants of 'unstructured branches' phenotype, milder (Ila) (pink brackets) and stronger (Ilb) (pink arrowheads). B The graph summarizes two independent experiments. Number of treated embryos are indicated for each column.

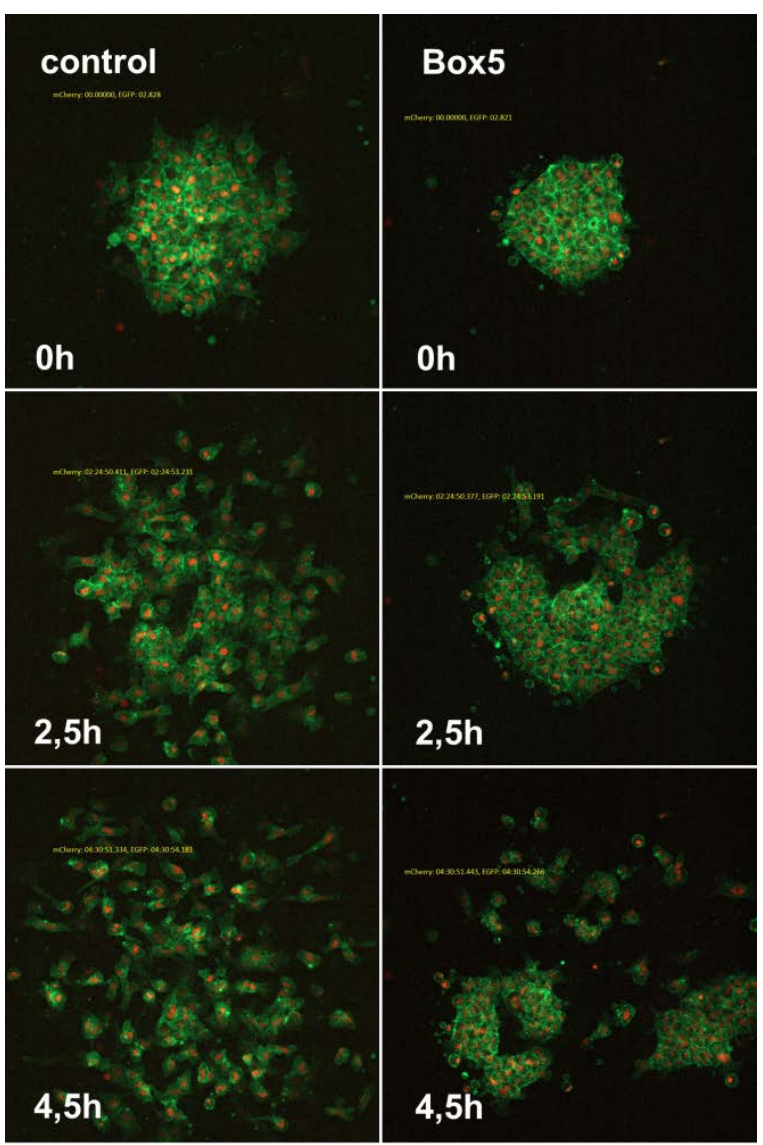

Figure.4.15.4. Box5 treatment limits migration of explanted NC cells. A Time-lapse images of control (left panels) and Box5-treated NC cells (right panels) at the start of imaging (0 hours) and after 2,5 and 4,5 hours. Cells were treated with $100 \mu \mathrm{M}$ Box 5 and DMSO as control. Control NC cells leave the cluster and perform single cell migration. Box5-treated NC cells show decreased motility. NC cells don't detachment from the cluster. The NC cluster becomes divided into smaller clusters, which migrate with limited motility. 
Finally we used IWP-2, which is an inhibitor of Wnt processing and secretion. IWP-2 inactivates Porcupine (Porcn), a membrane-bound O-acyltransferase, thereby selectively inhibiting palmitoylation of Wnt ligands. In the cell lines IWP-2 was shown to block all canonical Wnt-dependent biochemical changes including phosphorylation of the Lrp6 receptor and Dvl2 and B-catenin accumulation. This inhibition was not only specific for Wnt3a activity, but also for Wnt1 and Wnt2. In addition, IWP-2 was also able to decrease palmitoylation of Wnt5, regarded as "non-canonical" Wnt indicating that IWP-2 might be a global inhibitor of Wnt signaling (Chen et al., 2009). Interestingly, treatment of whole-embryos and explanted NC cells with IWP-2 resulted in migration defects observed already upon treatment with BIO and IWR-1. In whole-embryos analysis, migration defects resembling 'inhibited migration' and 'merged branches' phenotypes were observed (Fig.4.15.5AB) In explanted NC cells, treatment with IWP-2 caused only slightly decreased cell mobility, however formation of protrusion seemed to be more disturbed (Fig.4.15.6. Mov.6). The amount and severity of observed phenotypes were milder in comparison to BIO and IWR-1 treatment suggesting that IWP-2 might not be a potent modulator.

A

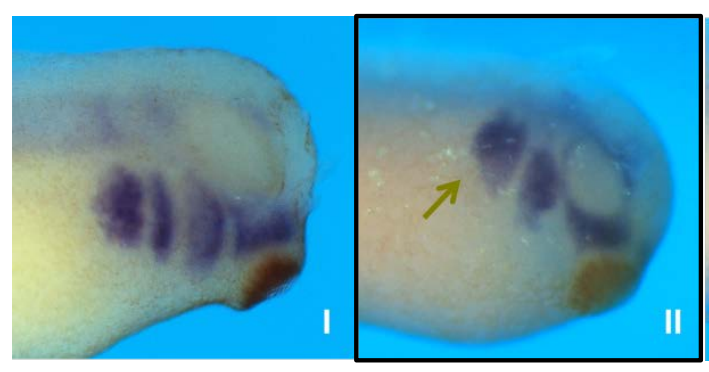

B

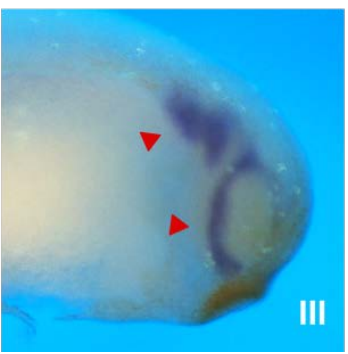

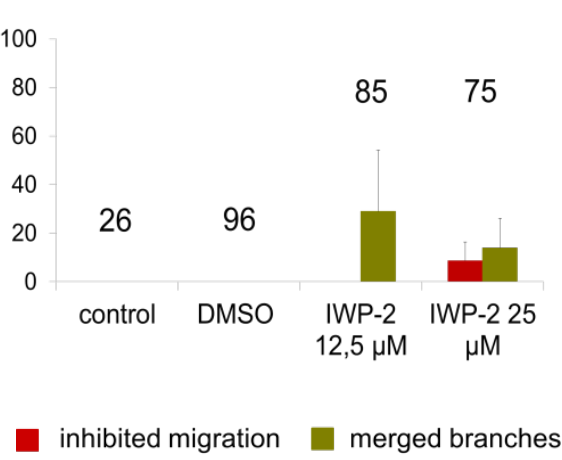

Figure.4.15.5. Global inhibition of Wnt signaling in whole embryos by treatment with IWP-2 inactivating Wnt processing and secretion. Wild type Xenopus embryos were treated with increasing concentrations of IWP-2 at NC premigratory stages or DMSO as a solvent control. A Panel shows an unaffected embryo (I), embryo with merged NC branches (II), embryo with inhibited NC migration (III). Green arrow marks merged posterior NC streams. Red arrows mark a weakly stained NC cluster in the premigratory position and the remaining mandibular branch. Black frame marks the most abundant phenotype $\mathbf{B}$ The graph summarizes three independent experiments. Standard errors of the means and number of treated embryos are indicated for each column. 


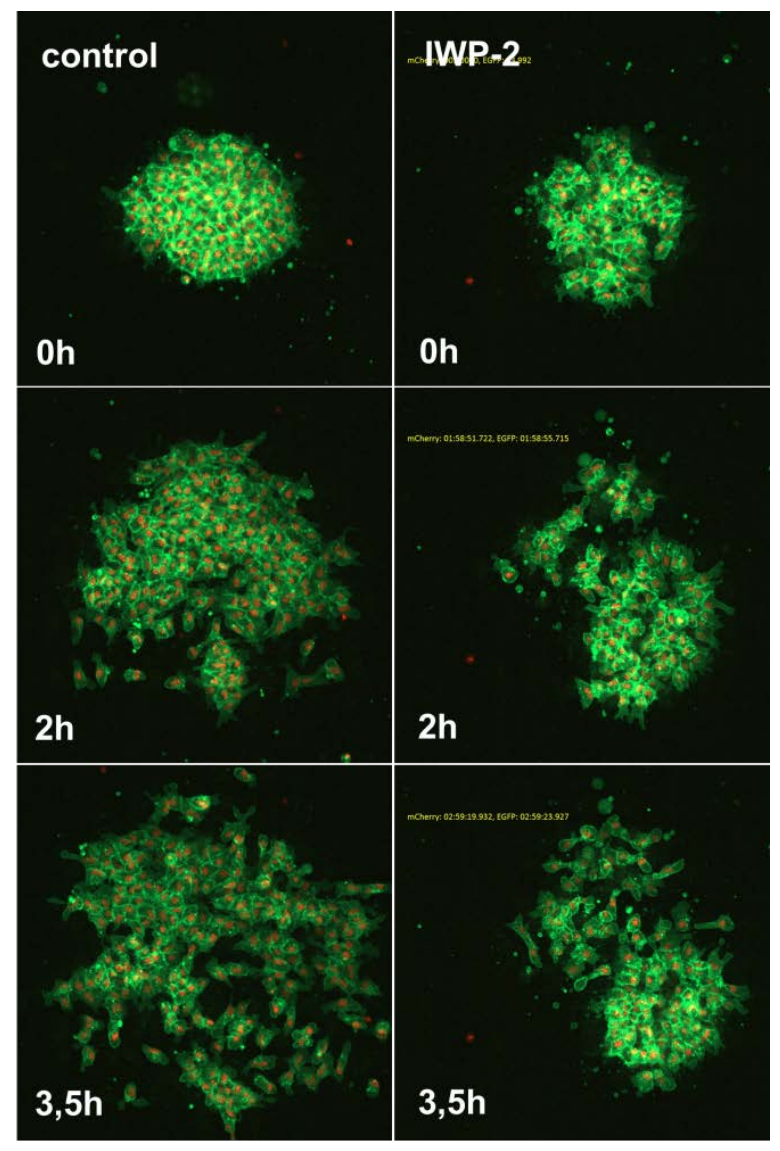

Figure.4.15.6. IWP-2 treatment slightly limits migration of explanted NC cells. A Time-lapse images of control (left panels) and IWP-2-treated NC cells (right panels) at the start of imaging ( 0 hours) and after 2 and 3,5 hours. Cells were treated with $1 \mu \mathrm{M}$ IWP-2 and DMSO as control. Control NC cells leave the cluster and perform single cell migration. IWP-2-treated NC cells show slightly decreased motility. NC cells detach partially from the cluster and migrate in the restricted area. 


\section{Discussion}

\section{Activation and inhibition of canonical Wnt signaling causes the same migration defects}

A requirement of canonical Wnt signaling in NC induction as well as in the formation of its derivatives has been well established, however, its function in NC migration remains in the initial phase of investigation. Taking advantage of chemical modulators and inducible constructs to activate or inhibit canonical Wnt signaling either at premigratory or migratory NC cell stages, we show that Wnt signaling needs to be tightly controlled to allow for proper NC migration. Intriguingly, our data demonstrate that up-regulation or inhibition of canonical Wnt signaling both lead to the same NC migration defects. Since similar defects are observed upon treatment of whole embryos at premigratory and migratory stages, this effect seems not to be restricted to a function in NC delamination, but likely causes defects in NC migration. Results obtained in explanted NC cells, where activation and inhibition of canonical Wnt signaling severely reduced the migration abilities of NC cells, confirm the in vivo studies.

\section{Role of canonical Wnt signaling in post-induction development of NC cells}

The concept that canonical Wnt signaling plays a role in post-induction NC development has been supported by a plethora of studies across vertebrates. Canonical Wnt signaling has been associated with development of craniofacial structures and NC derivatives including melanocytes and sensory neural cells (Dorsky et al., 1998; Brault et al., 2001; Hari et al., 2002; Lee et al., 2004; Lewis et al., 2004; Hari et al., 2012). In zebrafish, overexpression of ß-catenin in premigratory NC cells has been shown to promote pigment cell formation at the expense of neurons and glia, while inhibition of Wnt signaling using a truncated form of Tcf3 or a dominant-negative Wnt1 caused an opposite effect promoting neuronal fate at the expense of pigment cells (Dorsky et al., 1998). In accordance with these findings, conditional deletion of ß-catenin in premigratory mouse NC cells also prevented the formation of melanocytes (Hari et al., 2002). However, here sensory neurogenesis 
was also impaired. Another study in mouse, contradicting zebrafish data shows that sustained ß-catenin activity in NC cells promoted the generation of sensory neurons, but had no effect on melanocyte formation (Lee et al., 2004). A later study using different Cre-lines to activate ß-catenin signaling at specific time points during NC development proved a temporal separation in generation of NC derivatives where sensory neurons are determined earlier than melanocytes (Hari et al., 2012). Thus, even after migration there seem to be a clear-cut time windows where canonical Wnt signaling exerts certain functions in the development of NC cells. Interestingly, in none of these studies comprising in vitro and in vivo analysis in the mouse model system, any defects in NC migration have been observed (Brault et al., 2001; Hari et al., 2002; Lee et al., 2004). However, it is worth noting that sustained ß-catenin signaling in the mouse has been shown to prevent cranial NC cells from entering the branchial arches (Lee et al., 2004) indicating that NC migration should also be affected. In addition, the growing concern on the usage of mouse as a model system for the study of NC development supports the necessity to use complementary systems (Barriga et al., 2015). As an example, further usage of the Wnt1-Cre lines for conditional gene knockout seem to be doubtful, as its expression is not limited to the $\mathrm{NC}$ domain and was recently found to cause ectopic activation of Wnt signaling (Lewis et al., 2013).

\section{Role of canonical Wnt signaling in delamination of NC cells}

As opposed to the mouse data, our results as well as studies in the avian embryo support a role of canonical Wnt signaling in NC delamination and migration (BurstynCohen et al., 2004; de Melker et al., 2004). In chick, canonical Wnt signaling has been shown to facilitate G1/S transition and delamination of trunk NC cells (BurstynCohen et al., 2004). Inhibition of canonical Wnt signaling by expression of a dominant negative dsh, a dominat negative Lef1 or a ß-catenin-engrailed fusion protein prevented G1/S transition and inhibited NC delamination. Interestingly, activation of canonical Wnt signaling using $\mathrm{LiCl}$ or exogenous Wnt1 treatment was shown to inhibit in vitro migration of avian trunk NC cells (de Melker et al., 2004). Thus, these data provide evidence for a specific requirement of canonical Wnt signaling in different phases of post-induction NC development. NC delamination requires canonical Wnt activity, however later it needs to be downregulated to enable NC 
migration. The hypothesis that post-induction NC development is divided into distinct phases of canonical Wnt signaling activity is further supported by the time-course of nuclear localization of B-catenin, which is a hallmark of active canonical Wnt signaling. Analysis of the avian trunk NC cells emigrating from neural tube explants, revealed a subset of $\mathrm{NC}$ cells remaining in close proximity to the neural tube, most likely representing $\mathrm{NC}$ cells at the onset of migration, which showed nuclear localization of ß-catenin. On the contrary, in migrating NC cells ß-catenin was mainly localized to cell-cell contacts in association with $\mathrm{N}$-cadherin (de Melker et al., 2004). In this study, ß-catenin staining was analyzed in NC cells emigrating from trunk neural tube explants, and distinction between premigratory and migratory cells was based on their distance from the neural tube. In our approach we determined ßcatenin localization in cranial NC explants at premigratory stages versus explants that were cultured for extended time periods corresponding to migratory stages ( 3 or 7 hours respectively). Supporting that canonical Wnt signaling is still active before NC emigration, we detected nuclear ß-catenin in NC explanted from embryos at the premigratory stages, while $ß$-catenin in NC explants which underwent migration was mainly localized at cell-cell contacts. These data suggest that canonical Wnt needs to be active possibly to allow for delamination, but then has to be down-regulated during NC migration. Consistent with this, we find that BIO-treatment leads to an increase in nuclear ß-catenin levels in explanted NC cells, comparable to the level detected in NC explanted at premigratory stages contributing to defects in NC migration in vivo and in vitro. Since ß-catenin staining is still detected at cell-cell contacts of BIOtreated embryos and similar defects in NC migration are also observed after activation of Lef1- or Tcf3-signaling, these migration defects are likely the result of a transcriptional regulation and not simply caused by an absence of ß-catenin at cellcell contacts. A recent study in zebrafish analyzing the function of rabconnectin-3a, a v-ATPase interacting protein, has shown that loss of function of rabconnectin-3a leads to NC migration defects linked to elevated nuclear localization of B-catenin in comparison to controls (Tuttle et al., 2014). Conversely, at premigratory stages, ßcatenin was localized mostly to the nucleus in controls, but not in rabconnectin-3adeficient NC cells. Taken together, these data support a necessity of active canonical Wnt signaling at premigratory stages followed by a decrease in activity at migratory stages as shown both in avian and Xenopus NC cells (this study) and suggests that deregulation of canonical Wnt activity impairs NC migration. Further as 
Rabconnectin-3a deficient embryos showed alterations in the expression of celladhesion molecules - like for example an upregulation of the canonical Wnt-target gene cadherin-11, which we have previously shown to inhibit NC migration (Borchers et al., 2001) - this may explain how overstimulation of canonical Wnt signaling affects NC migration. Considering that endogenous nuclear ß-catenin peaks at premigratory stages and is subsequently reduced in migratory stages it is surprising that inhibition of canonical Wnt signaling leads to similar defects in migration as ectopic activation. Possibly, migrating NC cells retain a basal level of canonical Wnt signaling, which may be affected by our inhibitory approaches. In addition, a reduction in overall cytoplasmic levels may also reduce ß-catenin at sites of cell-cell contacts and thereby account for defects in NC migration. This may explain how IWR1, which stabilizes axin and thereby reduced ß-catenin levels, may affect NC migration. However, overexpressions of a dominant negative Tcf3 construct inhibiting Wnt signaling downstream of ß-catenin also caused NC migration defects, suggesting that defects are caused by transcriptional changes rather than a transcriptionindependent direct modulation of ß-catenin adhesion complexes.

\section{Interplay of NC-specifiers}

Time-laps images provide information about the initial changes in migration behavior of NC cells upon modulation of canonical Wnt pathway allowing assessment of general cell morphology, adhesiveness, motility and formation of cell protrusion. On the contrary, manipulation of canonical Wnt signaling in the whole embryos provides more global information about the ability to perform migration, formation of $\mathrm{NC}$ streams and expression of NC markers. In a two-step model of NC induction, which has been proposed in Xenopus, zebrafish and chick systems (Stuhlmiller and GarciaCastrio 2012; Faure et al., 2002, Patthey et al., 2009), canonical Wnt signaling has been shown to be responsible for activation of BMP signaling in the second step of NC induction. In accordance with this two-step model inhibition of BMPs during gastrulation allows other signaling pathway (Wnts, FGFs) to initially specify NC, while later activation of BMPs at neurula stages maintains NC fate in the neural plate borders (NPB) and is followed by expression of NC specifiers including: Snail2, FoxD3, Sox9/10, Twist, cMyc, and Ap2. NC specifiers establish a gene regulatory network, which governs further development of NC cells. Activation of canonical Wnt 
signaling could therefore cause a dysregulation in the expression of those specifiers and impairment in either delamination or NC migration. Indeed, treatment with BIO and $\mathrm{LiCl}$ (summarized in the supplement, only Twist-positive NC cells) as well as overexpression of Lef1/Tcf3 activator constructs in vivo inhibited NC cell migration or disturbed the formation of separate NC streams. This was observed for Twist- and Sox10-positive NC cells respectively. However, while in general the Sox10 expression was rather increased and branches seemed to merge to a broad Sox10positive cluster, the Twist expression appeared reduced. The preliminary RT-PCR data (summarized in the supplement) seem to confirm the in vivo data. Twist expression is initiated in an anterior to posterior sequence, beginning in the presumptive mandibular crest and subsequently expanding to the hyoid and two branchial NC segments, which could explain an increasing reduction of Twist-positive cells in NC streams along anterior-posterior axis. The mandibular branch is the least affected, found at its final location around or under the developing eye in contrast to the II branchial arch either being merged with the I branchial arch or missing, indicating that the mandibular branch migrates earlier making it less prone to activation of canonical Wnt signaling. Moreover, Twist has been shown to regulate NC migration by repression of E-cadherin in delaminating cells. Knockdown of Twist leads to an upregulation of E-cadherin and subsequently inhibition of cell dispersion (Barriga et al., 2013). In vitro monitoring of NC explants treated with BIO or overexpressing Lef1 activator construct revealed an elevated cell adherence. NC cells were unable to perform single cell migration and remain joined together in NC clusters. Since E-cadherin is a target gene of canonical Wnt signaling it's upregulation causing impaired migration could also lead to loss of Twist expression in cells unable to move. NC cells treated with BIO showed additionally disturbances in membrane dynamics, which could be credited not only to transcriptional changes, but also dysregulation of the ß-catenin pool at the cell membrane.

Expression of Sox10 during induction is stimulated by canonical Wnt signaling. Analysis of Sox10 gene in mouse revealed multiple regulatory regions including Lef/Tcf binding sites which are responsible for controlling expression of Sox10 (Werner et al., 2007). Sustained activation of canonical Wnt signaling could therefore explain enhanced expression of Sox10 in the migratory NC cells. Furthermore, our preliminary data summarized in the supplement, show that expression of AP2 and Krox-20 upon chemical modulation of canonical Wnt signaling differs from the 
expression of Twist and Sox10 indicating that each population of NC cells might exhibit a distinct requirement for canonical Wnt signaling. However, more precise conclusion will be possible after analysis of NC migration defects cause by modulation of Lef1/Tcf3 signaling.

\section{Lef1 and Tcf3 signaling}

Both Lef1 and Tcf3 have been reported to be expressed in the NC cells (Molenaar et al., 1998). In tailbud stages, Lef1 has been shown to be present at higher levels in comparison with Tcf3, while in tadpole stages expression of Lef1 is less prominent than that of Tcf3 providing an additional explanation for different frequencies of defects cause by overexpression of Lef1-GR and Tcf3-VP16-GR activator constructs at premigratory versus migratory stages in Twist-positive $\mathrm{NC}$ and indicating opposite requirements for Lef1/Tcf3 expression during development of NC cells. Since several studies have shown that Lef/Tcf's have overlapping but also unique expression patterns as well as different functions in canonical Wnt pathway, it is likely that Lef1 and Tcf3 signaling represtents distinct pathways in the development of NC cells (Cadigan and Waterman 2012, Staal and Clevers, 2000). Furthermore, defects in the migration of NC cells upon overexpression of Tcf3 $\triangle \mathrm{C}$-GR inhibitor construct in later stages could be linked not only with NC migration, but also with formation of Twistpositive NC derivatives since its expression (Yoon et al., 2011) together with others Tcf's including; Tcf1 (Roël et al., 2003.), Tcf12 (Yoon et al., 2011) and Tcf21 (Simrick et al., 2005) have been shown in the branchial arches at late talibud stages. Overexpression of EnR-Lef1-GR inhibitor construct in different concentrations and at different stages of NC development did not cause any defects in migration of NC cells implicating that most likely EnR-Lef1-GR is not functional (summarized in supplement). Thus, further investigation of Lef1 signaling in NC cells migration would require a generation of another dominan-negative GR-construct.

\section{Lef/Tcf signaling as a platform for canonical and non-canonical Wnt signaling}

The necessity to down-regulate ß-catenin levels at the onset of NC migration and expression of ß-catenin effectors, namely Lef1/Tcf3 in the NC cells seems to be contradictory, however, Lef1/Tcf3 could provide a platform to integrate canonical and 
non-canonical Wnt signaling. A B-catenin independent activity of Lef1/Tcf1 and expression of target genes associated with canonical Wnt pathway involving direct recruitment of ATF2 in Human Hematopoietic tumor cells has been reported (Sprowl, and Waterman 2013, Grumolato et al., 2013). Since ATF2 is activated in response to signals that converge on proteins involved in cell migration including JNK, it is tempting to speculate that Lef1/Tcf3 could mediate expression of genes being targets of both Wnt pathways. Many of NC specifiers including Snai2 and Sox10 have Lef/Tcf binding motives. It could be possible that expression of various NC-specific genes during migration would be maintained by ß-catenin-independent Lef/Tcf signaling, while down-regulation of $B$-catenin before NC migration would serve to restrict the number of target genes expressed during NC migration and potentially also explain less severe effect of IWR1 treatment in comparison to overexpression of Tcf3 inhibitor.

\section{Perspectives}

For the time being, the most crucial question is why activation as well as inhibition of canonical Wnt signaling results in a comparable defects in migration of Twist- and Sox10-positive NC cells. So far, the preliminary RT-PCR data (summarized in supplement) show that modulation of Lef1/Tcf3 signaling increases expression of cJun and Brachyury suggesting a possible contribution of those genes in the observed defects. Therefore, future studies should employ transcriptome analysis to precisely reveal how canonical Wnt signaling controls migration of different NC populations. 


\section{Supplement}

\subsection{Functionality of Lef1/Tcf3 constructs in double axis assay}

A
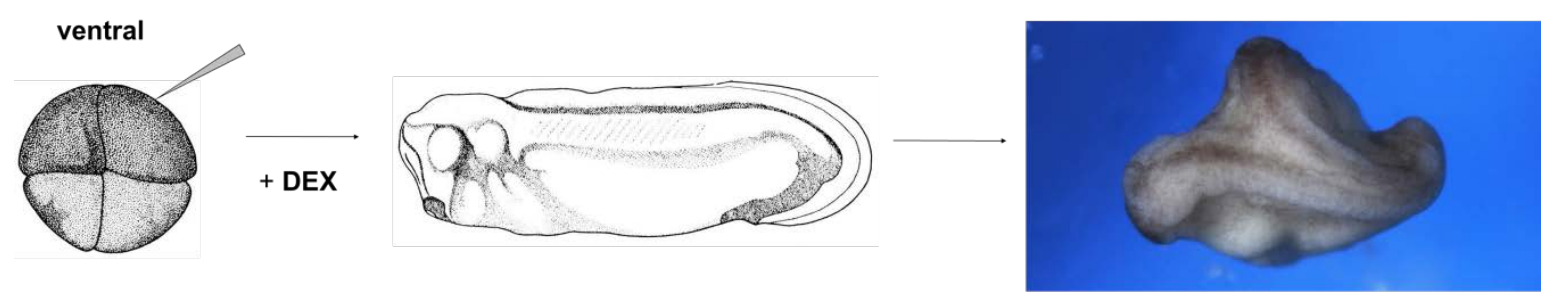

axis duplication
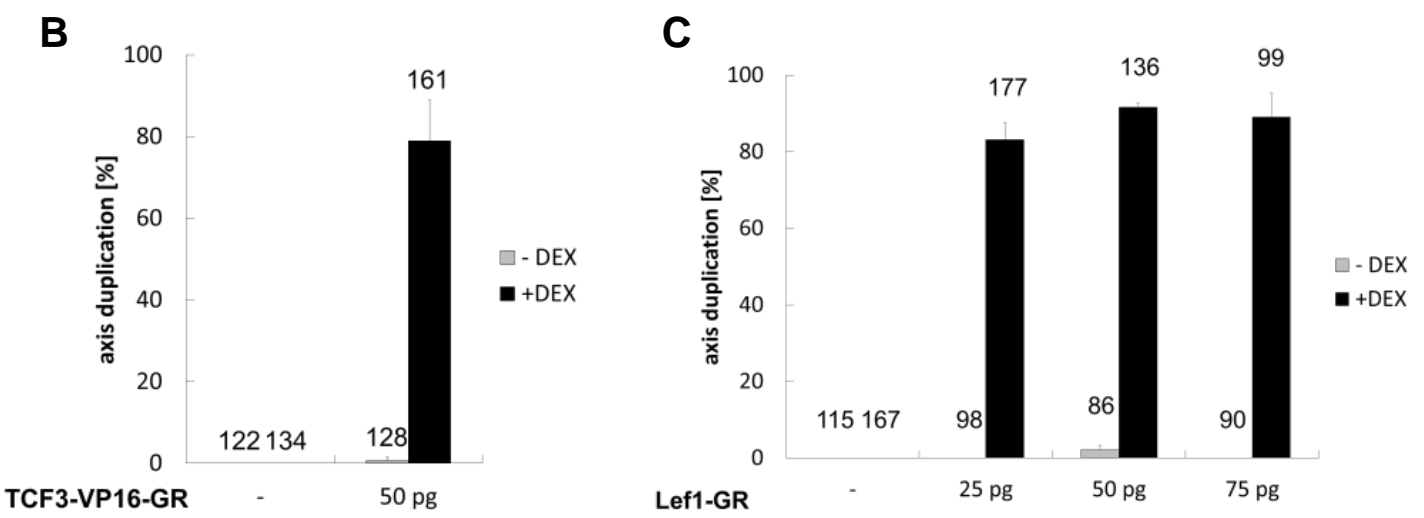

Supplement figure 6.1. Functionality of Lef1/Tcf3 constructs in double axis assay. A Experimental procedure leading to dorsalization of Xenopus embryos. RNAs of Lef1- GR and Tcf3-VP16 were injected marginally into one ventral blastomere at the 4-cell stage. Embryos were cultured with Dexamethasone starting at the 16-cell stage (the solution was changed to normal medium at gastrula stages 10-11) and induction of double axis was analyzed at early tailbud stages. B Graph summarizing the percentage of secondary axes of 3 independent experiments of embryos injected with 50 pg of Tcf3-VP16-GR RNA. Numbers of injected embryos and standard error of the means are indicated for each column. C Graph summarizing the percentage of secondary axis in 3 independent experiments after injection of increasing concentrations of the Lef1-GR construct. 


\subsection{Functionality of Lef1/Tcf3 constructs in ventralization axis assay}
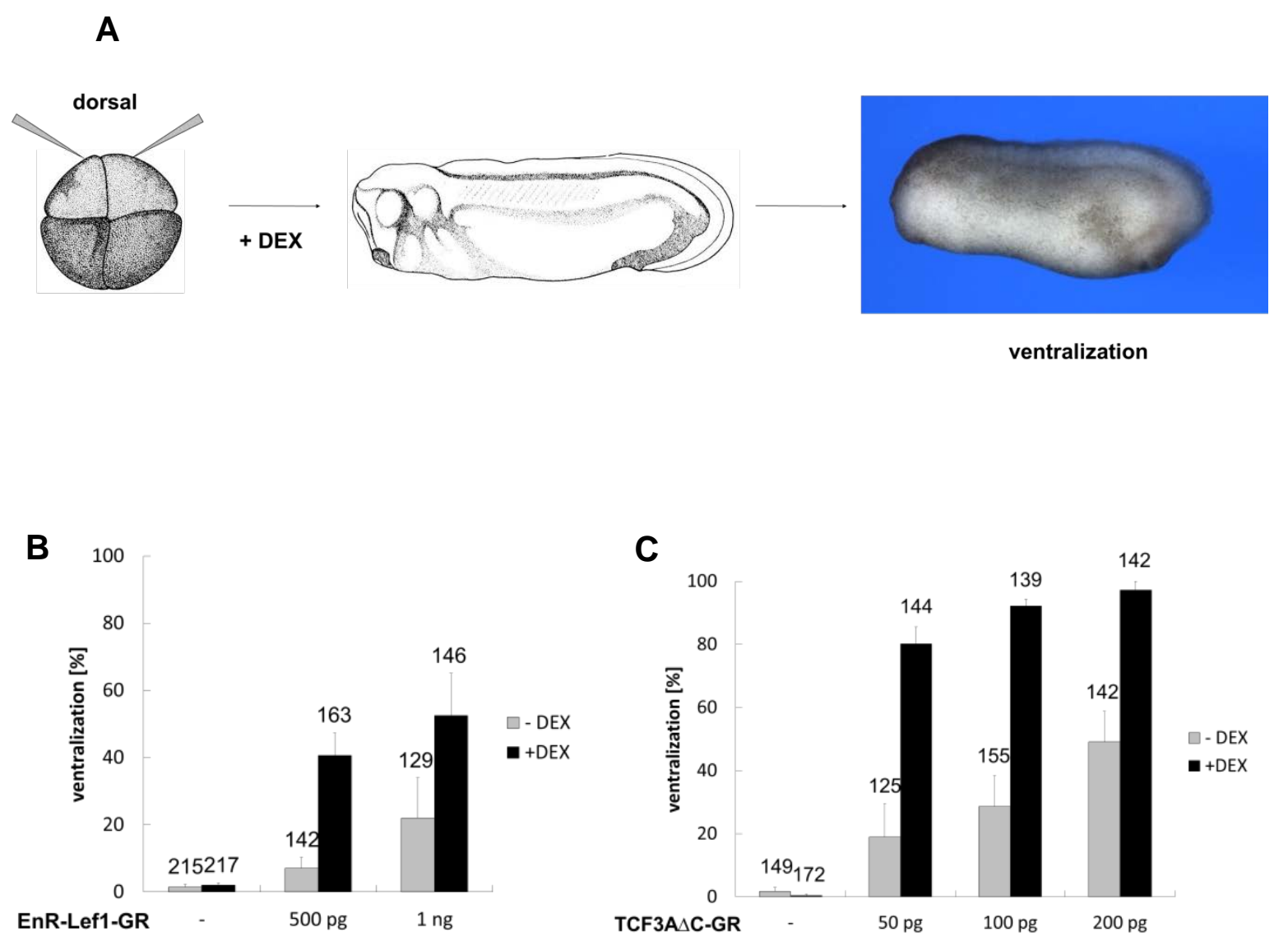

Supplement figure 6.1. Functionality of Lef1/Tcf3 constructs in ventralization axis assay A Experimental procedure leading to ventralization of Xenopus embryos. RNAs coding for EnRLef1-GR or Tcf3 $\Delta$ C-GR were injected into both dorsal blastomeres at the 4-cell stage, treated with Dexamethasone starting at the 16-cell stage (the solution was changed to normal medium at gastrula stages 10-11) and ventralized embryos were scored at early tailbud stages. The analysis of EnR-Lef1-GR and Tcf3 $\Delta \mathrm{C}$ was done by Dr. Juliane Melchert. B Graph summarizing the percentage of ventralized embryos in 3 independent experiments after injection of EnR-Lef1-GR. At low concentrations EnR-Lef1-GR overexpression did not show strong ventralizing activity and at high concentrations, it showed activation even in the absence of Dexamethasone. C Graph summarizing the percentage of ventralized embryos of 3 independent experiments in embryos injected with increasing concentrations of Tcf3 $\triangle C$-GR RNA. Numbers of injected embryos and standard error of the means are indicated for each column. Tcf3 $\triangle \mathrm{C}-\mathrm{GR}$ is a potent ventralizing agent, however, also active to some extent in the absence of Dexamethasone 


\subsection{Inhibition of canonical Wnt pathway by overexpression of EnR-Lef1-GR does not cause any defects in migration of Twist-positive NC cells}

Overexpression of EnR-Lef1-GR in the concentration with no significant background (50 pg) at the premigratory and migratory NC stages, as well as in the higher concentration $(500 \mathrm{pg})$ at the premigratory NC stages did not cause any defects in migration of the Twist-positive NC cells (Supplement Fig.6.3).

A

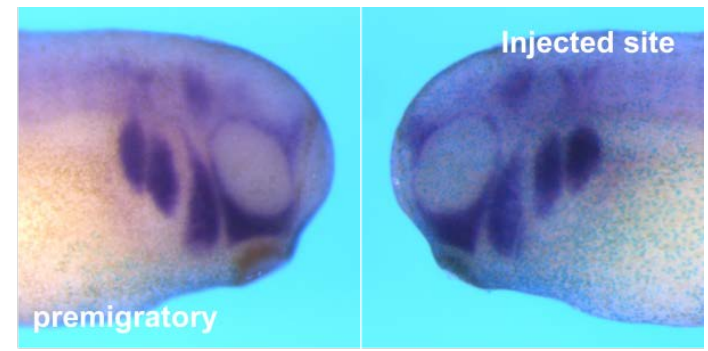

B

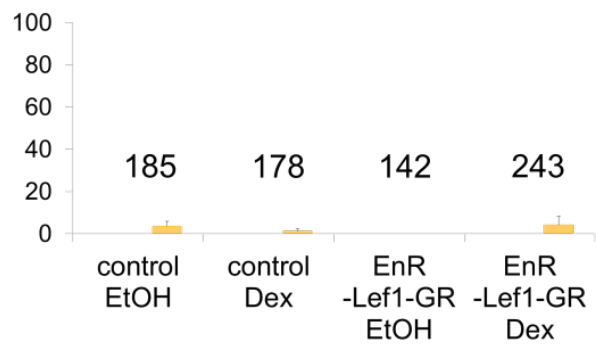

C

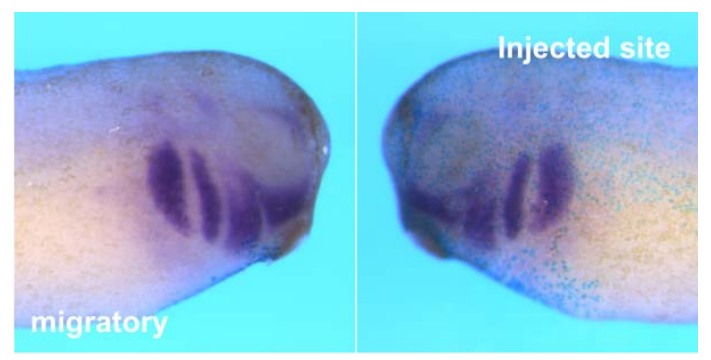

100
80
60

40

\begin{tabular}{|c|c|c|c|}
\hline 214 & 220 & 171 & 255 \\
\hline $\begin{array}{c}\text { control } \\
\text { EtOH }\end{array}$ & $\begin{array}{c}\text { control } \\
\text { Dex }\end{array}$ & $\begin{array}{c}\text { EnR } \\
\text {-Lef1-GR } \\
\text { EtOH }\end{array}$ & $\begin{array}{c}\text { EnR } \\
\text {-Lef1-GR } \\
\text { Dex }\end{array}$ \\
\hline
\end{tabular}

E
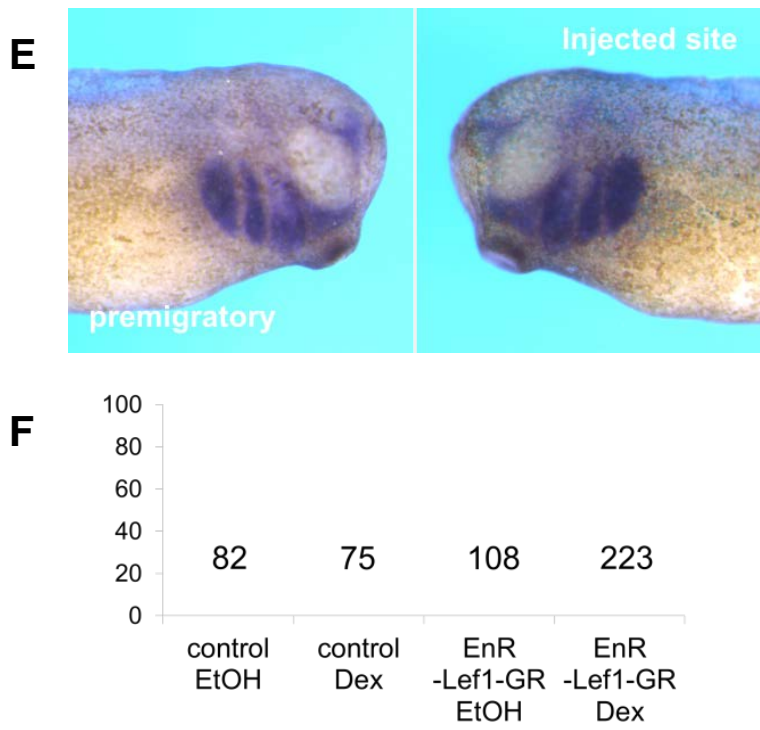

Supplement figure.6.3. Overexpression of EnR-Lef1-GR does not cause any defect in migration of Twistpositive NC cells. A Overexpression of EnR-Lef1-GR at premigratory NC stages $(50 \mathrm{pg})$. C Overexpression of EnR-Lef1-GR at migratory NC stages (50 pg). E Overexpression of EnR-Lef1-GR at premigratory NC stages (500 pg). B,D,F Each graph summarizes three independent experiments. Standard errors of the means and number of treated embryos are indicated for each column 


\subsection{Activation of canonical Wnt signaling by $\mathrm{LiCl}$ treatment causes defects in migration of NC cells}

The role of $\mathrm{LiCl}$ in activation of canonical Wnt signaling is well established (Klein and Melton 1996). As BIO, it activated canonical Wnt signaling by inhibiting GSK3ß. Treatment with $\mathrm{LiCl}$ was performed in the same maner as with the $\mathrm{BIO}$ and caused the same phenotypes observed already upon BIO treatment. Treatment at the premigratory stages resulted mostly in occurrence of 'inhibited migration' phenotype (Supplement Fig.6.4A), while in the migratory treatment 'merged braches' phenotype was the most prominent (Supplement Fig.6.4). On the contrary to the $\mathrm{BIO}$, $\mathrm{LiCl}$ did not cause 'reduced branches', which was observed in embryos treated with BIO:

A

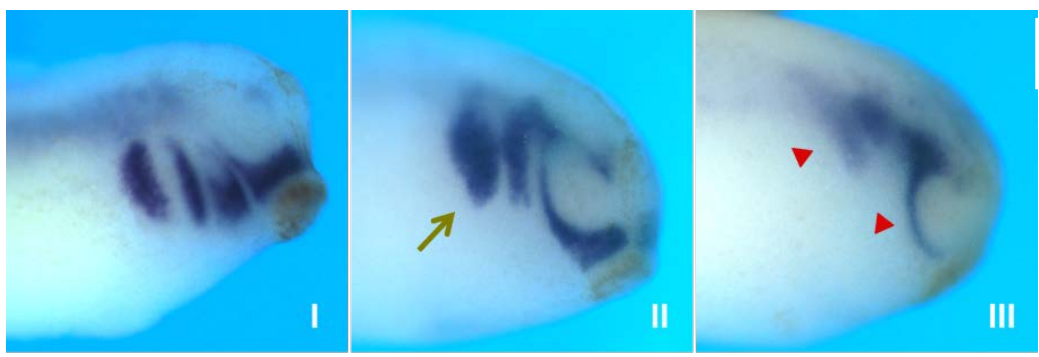

C

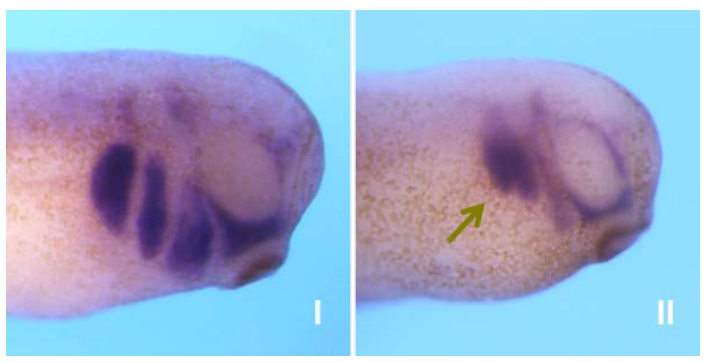

B

60

40

20

0

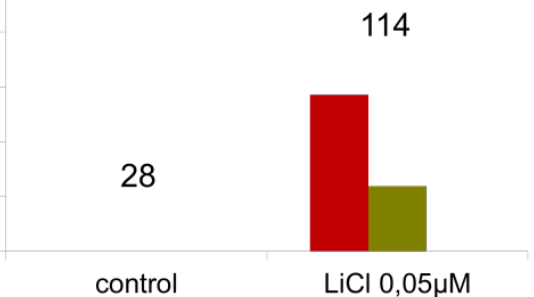

control

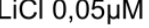

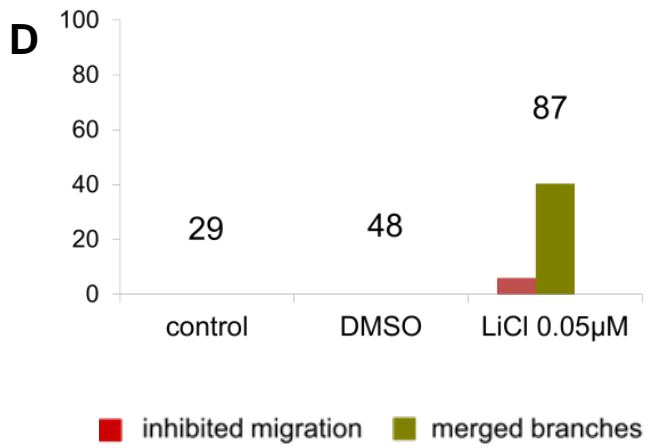

Supplement figure.6.4. Activation of canonical Wnt signaling using $\mathrm{LiCl}$ at premigratory and migratory NC cell stages affects NC migration. Wild type Xenopus embryos were treated with $\mathrm{LiCl}$ at $\mathrm{NC}$ premigratory or migratory stages or with DMSO as a solvent control. A The panel shows the different phenotypes observed after $\mathrm{LiCl}$ treatment at premigratory stages. Phenotypes are grouped by increasing severity: unaffected embryo (I), embryo with merged NC branches (II), embryo with inhibited NC migration and reduced Twist expression (III). Green arrow marks merged posterior NC streams. Red arrows mark a weakly stained NC cluster in the premigratory position and the remaining mandibular branch. B Graph summarizing the percentage of phenotypes observed in 1 experiment. C The panel shows the different phenotypes observed after LiCl treatment at migratory stages. Phenotypes are grouped by increasing severity: unaffected embryo (I), embryo with merged NC branches (II). Very few embryos showed 'inhibited migration phenotype'. D Graph summarizing the percentage of phenotypes observed in 1 experiment. 


\subsection{Modulation of non-canonical Wnt pathway affects migration of Sox10- positive NC cells}

Similary to Twist-positive NC cells, inhibition of non-canonical Wnt signaling using SP600125 causes an inhibited migration of Sox10-positive NC cells as well as a 'comb-shaped' NC streams where all streams where formed, but remained in close proximity with each other and seemed to be compact. Additionally, in the 'comshaped' phenotype the typical tear-shape of NC branches was lost and otic placode was not easily distinguishable as in the control. In 'inhibited migration' phenotype otic placide was not visible (Supplement Fig.6.5A). As in the case of Twist-positive NC, treatment with SP600125 did not result in reduction of Sox10 staining.

A

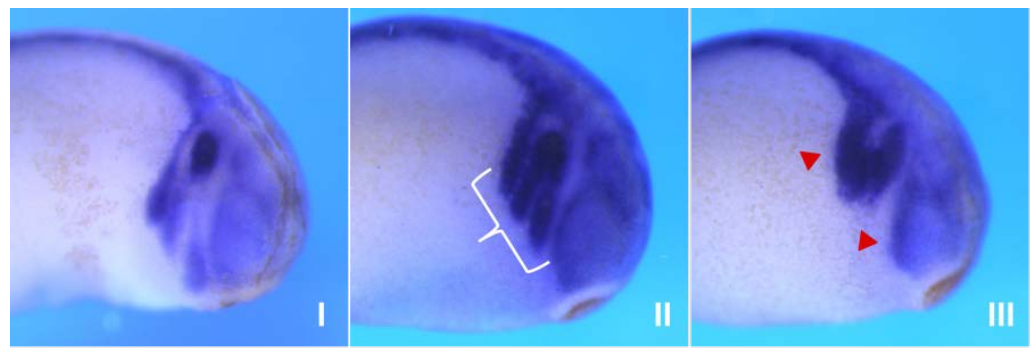

B

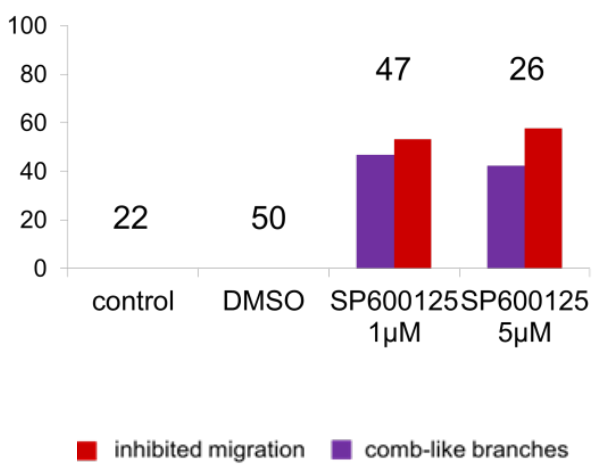

Supplement figure.6.5. Inhibition of non-canonical Wnt signaling in whole embryos by treatment with the JNK inhibitor SP600125 causes defects in migration of Sox10-positove NC cells. Wild type Xenopus embryos were treated with increasing concentrations of SP600125 at NC premigratory stages or DMSO as a solvent control. A Panel shows an unaffected embryo (I), 'comb-like' phenotype with compacted NC branches (II) (white bracket) and 'inhibited migration' phenotype where clusters of NC remaining at the proximity of the neural tube (III) (red arrowhead). B The graph summarizes 1 experiment. Number of treated embryos are indicated for each column. 


\subsection{Determination of Twist and Sox10 expression by RT-PCR}

Since Twist- and Sox10-positive NC cells seemed to be reduced and expanded respectively through modulation of Lef1/Tcf3 signaling we performed RT-PCR to reveal if activation and inhibition of Lef1/Tcf3 signaling has an effect on Twist and Sox10 expression levels. In the case of embryos overexpressing Lef1/Tcf3 constructs a clear reduction of Twist expression in comparison to controls was observed. Interestingly, all constructs including activators and inhibitors caused Twist reduction. On the contrary, reduction of Sox10 was not observed upon modulation of Lef1/Tcf3 signaling. Noteworthy, expression of Sox10 is slightly increased with a clear rise upon overexpression of Tcf3 $\Delta$ C-GR (Supplement Fig.6.6A,B)

A

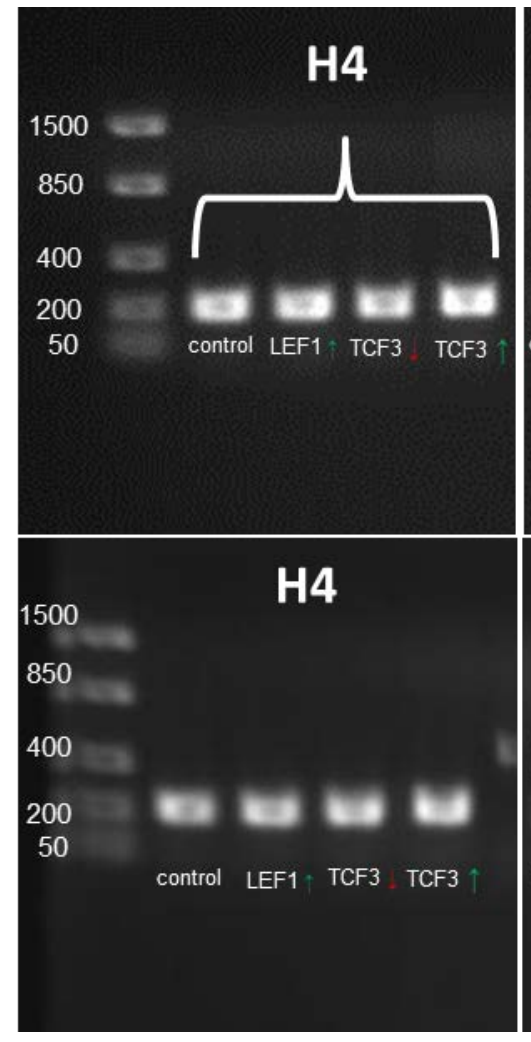

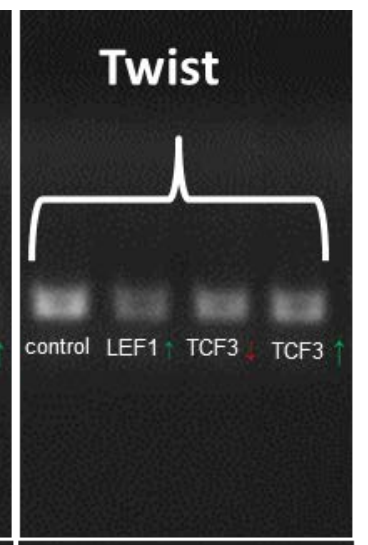

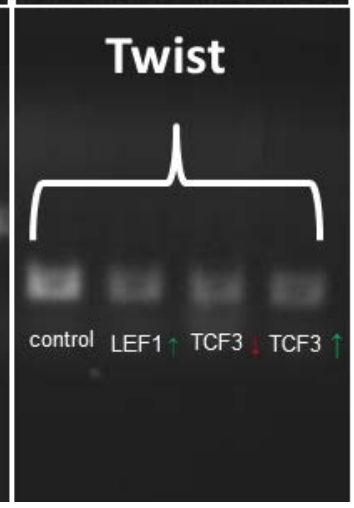

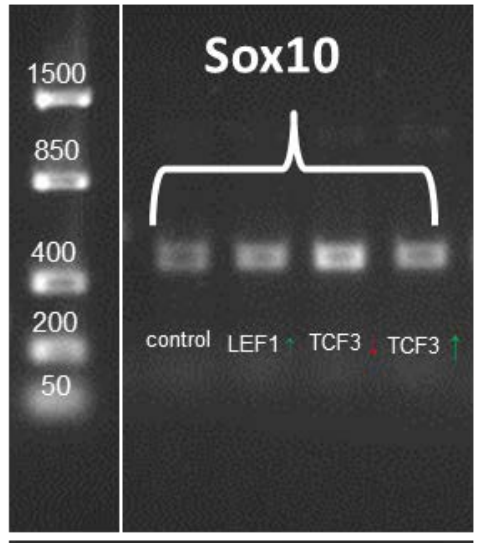

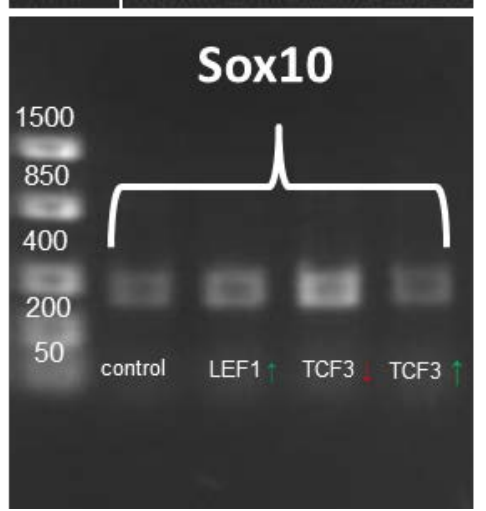

Supplement figure 6.6. Expression of Twist and Sox10 upon modulation of Lef1/Tcf3 signaling. A,B Wild type Xenopus embryos were injected with 25pg of Lef-1-GR, Tcf3 $\Delta$ C-GR and Tcf3-VP16-GR at 2 blastomers at 2cell stage embryo. Embryos were treated with Dexamethasone at the premigratory stages and processed for RTPCR. 10 embryos were processed for each treatment. Modulation of Lef1/Tcf3 signaling caused clear reduction of Twist expression and slightly elevated Sox10 expression with significant increase upon Tcf $3 \Delta \mathrm{C}-\mathrm{GR}$ overexpression. $\mathrm{H} 4$ served as a control. Figure summarizes 2 independent experiments. 


\subsection{Determination of c-Jun and Brachury expression by RT-PCR}

Both c-Jun and Brachyury are recognized as target genes of canonical Wnt signaling. Expression of C-Jun is found in premigratory as well as migratory NC cells (Knöchel et al., 2000). On the contrary, Brachyury expression is localized to the circumblastopolar collar and notochord at NC-induction stages and later to the eye, otic placode and head region at NC-differentiation stages (Cast et al., 2012). Overexpression of Lef1-GR caused a significant increase in expression of c-Jun and Brachyury. Overexpression of $\operatorname{Tcf} 3 \Delta \mathrm{C}-\mathrm{GR}$ also slightly elevated the expression of both genes (Supplement Fig.6.7). It is tempting to speculate that phenotypes observed upon modulation of Lef1/Tcf3 pathway are caused by dysregulated expression of c-Jun and Brachyury, however, the final conclusion will be only possible after performing transcriptome analysis of explanted NC overexpressing Lef1/Tcf3 constructs.

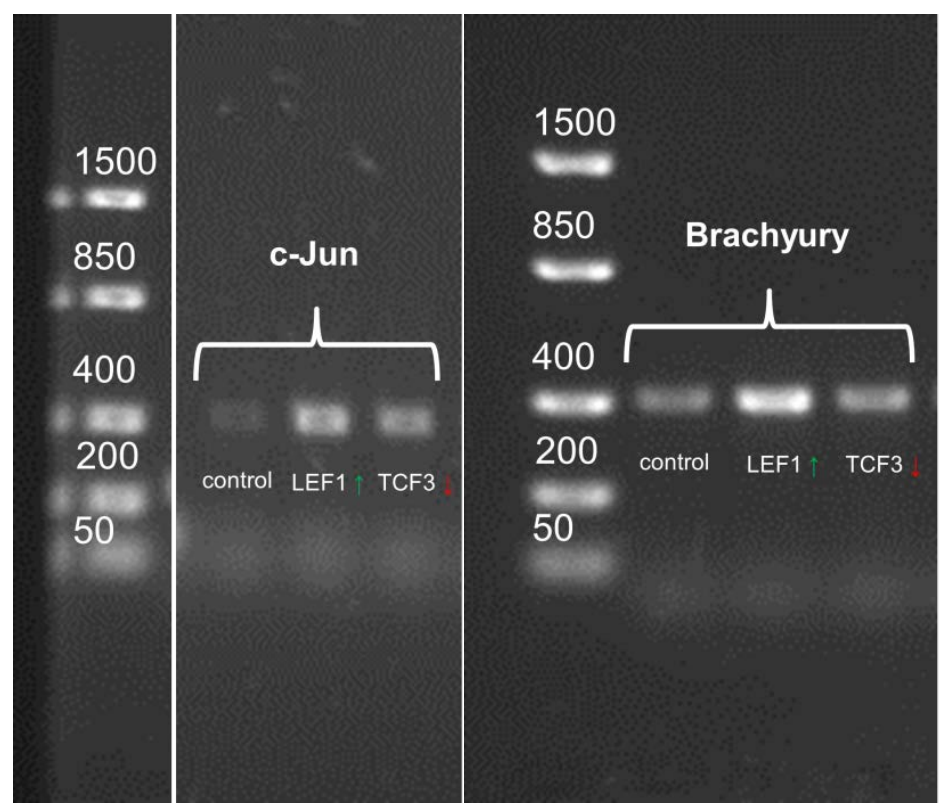

Supplement figure 6.7. Expression of c-Jun and Brachyury upon modulation of Lef1/Tcf3 signaling. Wild type Xenopus embryos were injected with 25pg of Lef-1-GR and Tcf3 $\Delta \mathrm{C}-\mathrm{GR}$ at 2 blastomers at 2-cell stage embryo. Embryos were treated with Dexamethasone at the premigratory stages and processed for RT-PCR. 10 embryos were processed for each treatment. Overexpression of Lef1-GR causes increase in expression of c-Jun and Brachyuyr. Figure summarizes experiment. 


\subsection{Effects of chemical modulation on AP2- and Krox20-positive NC cells}

In addition to monitoring the migration behavior of Twist- and Sox10-positve NC upon chemical modulation we also investigated migration of AP2- and Krox20-positive NC cells. In the case of the AP2-positive NC cells, activation of canonical Wnt signaling by BIO treatment at post-induction stages causes the 'reduced branches' phenotype observed already for Twist-positive cells. NC cells were able to migrate away from the prospective neural tube but the AP2-positive staining was considerably reduced. The more severe 'inhibited migration' phenotype defined as a small cluster of NC cells remaining at the premigratory position was not observed at all (Supplement Fig.6.8A,B). Interestingly, inhibition of canonical Wnt signaling by IWR1 treatment did not cause any defects in NC migration indicating that canonical Wnt signaling needs to be attenuated at the post-induction phase and is not required for the proper migration of the AP2-positive population of NC cells. In the case of Krox-20-positive NC population, neither activation nor inhibition of canonical Wnt signaling by chemical modulation caused defects in migration indicating that canonical Wnt might not play any role in their post-induction development (Supplement Fig.6.8C,D).

A

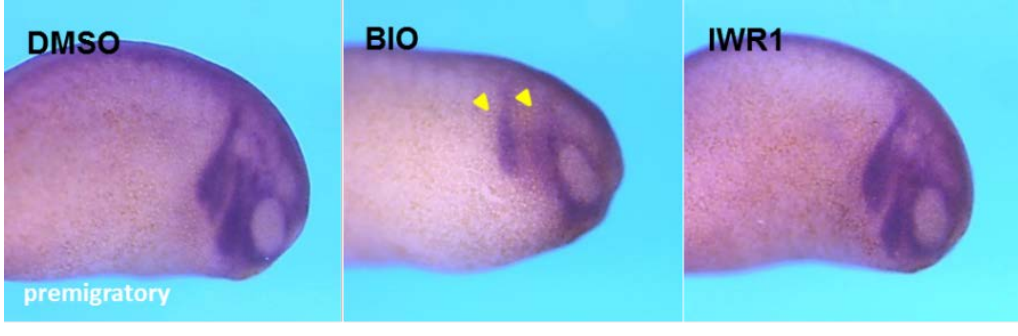

B
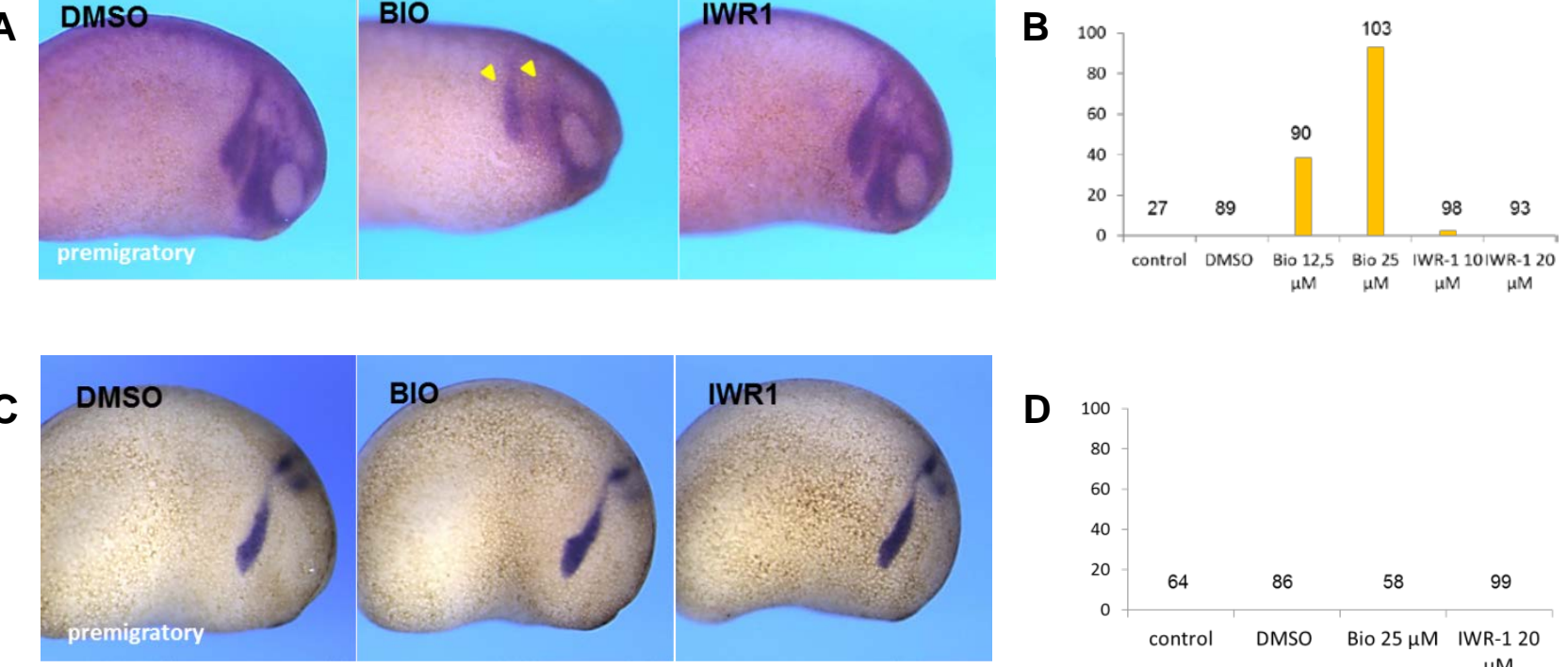

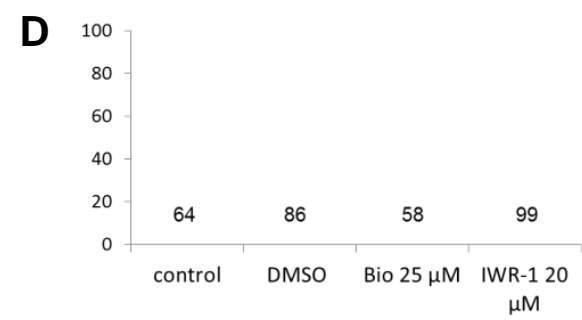

Supplement figure 6.6. Effect of chemical modulation on AP2- and Krox20-positive NC cells Wild type Xenopus embryos were treated with BIO or IWR1 at premigratory NC stages and DMSO treatment served as a solvent control. A The panel shows phenotypes observed after BIO and IWR1 treatments at premigratory stages. Left panel; control embryo. Middle panel; embryo showing 'reduced branches', the only phenotype observed upon BIO treatment. Right panel; embryo showing no NC migration defects after IWR1 treatment. B Graph summarizing 2 independent experiments. C Panel shows no defects observed after BIO and IWR1 treatment on Krox-20-positive NC cells D Graph summarizing 2 independent experiments. 


\subsection{Time-laps images}

Mov.1 BIO treatment inhibits in vitro migration of NC cells. Control NC explant (left panel) disassembles completely by frame 80th corresponding to approximately $2.45 \mathrm{~h}$ (out of $6 \mathrm{~h}$ ). NC cells detach from the cluster and perform single cell migration. BIO-treated NC cells (right panel) form initially proper lamellipodia, however, these are lost immediately upon detachment from the NC cluster and NC cells remain in close proximity to the explant.

\section{Mov.2 Lef1-GR inhibits in vitro migration of NC cells}

Control NC cells (left panel) disassemble completely by frame 100th, corresponding to approximately $3 \mathrm{~h}$ (out of 7 ). NC cells are able to detach from the cluster and perform single cell migration. NC cell overexpressing Lef-1-GR (right panel) show a strong inhibition of migration. NC cells form proper protrusion, but remain joined together and are not able to leave the cluster.

\section{Mov.3 IWR1 treatment inhibits in vitro migration of NC cells}

Control NC clusters disassemble completely by frame 50th, corresponding to approximately $3.40 \mathrm{~h}$ (out of 15). NC cells are able to detach from the cluster and perform single cell migration. IWR1-treated NC cells (right panel) leave the cluster efficiently. Initially cells form proper lamellipodia, which are partially retracted after leaving the cluster. Cells continuously leave the NC clusters, but due to their decreased mobility they remain in close proximity.

\section{Mov.4 Tcf3 $\Delta \mathrm{C}-\mathrm{GR}$ inhibits in vitro migration of NC cells}

Control NC clusters disassemble completely by frame 100th, corresponding to approximately $3 \mathrm{~h}$ (out of 7 ). NC cells are able to detach from the cluster and perform single cell migration. NC cells overexpressing Tcf3 $\Delta \mathrm{C}-\mathrm{GR}$ (right panel) form proper protrusion, but remain joined together for a prolonged time period until they finally detach.

Mov.5 SP600125 and Box treatment inhibits in vitro migration of NC cells Control NC explant (left panel) disassembles completely by frame 125th corresponding to approximately $4 \mathrm{~h}$ (out of $8 \mathrm{~h}$ ). NC cells detach from the cluster and 
perform single cell migration. Box5-treated NC cells have limited ability to migrate out from the NC cluster. NC cells migrate as smaller cluster (right panel). SP600125treated NC cells (middle panel) form proper lamellipodia, however, these are unable to detachment from the NC cluster and NC cells remain stick together.

Mov.6 IWP-2 treatment slightly limits in vitro migration of NC cells Control NC explant (left panel) disassembles completely by frame 80th corresponding to approximately $2.45 \mathrm{~h}$ (out of $6 \mathrm{~h}$ ). NC cells detach from the cluster and perform single cell migration. IWP-2-treated NC cells (right panel) form initially proper lamellipodia, however, these are partially lost upon detachment from the NC cluster and NC cells remain in closer proximity to the explant 


\section{References}

Aaku-Saraste E, Hellwig A, Huttner WB (1996) Loss of occludin and functional tight junctions, but not ZO-1, during neural tube closure-remodeling of the neuroepithelium prior to neurogenesis. Developmental Biology 180, 664-679.

Aberle H, Bauer A, Stappert J, Kispert A, Kemler R (1997) B-Catenin is a target for the ubiquitin-proteasome pathway.EMBO J 16: 3797-3804.

Acloque H, Adams MS, Fishwick K, Bronner-Fraser M, Nieto A (2009) Epithelial mesenchymal transitions: the importance of changing cell state in development and disease. The Journal of Clinical Investigation 119 (6).

Adams RH, Diella F, Hennig S, Helmbacher F, Deutsch U, Klein R (2001) The cytoplasmic domain of the ligand ephrinB2 is required for vascular morphogenesis but not cranial neural crest migration. Cell 104, 57-69.

Adler PN (2002) Planar signaling and morphogenesis in Drosophila. Dev. Cell 2, 525535.

Adler PN, Krasnow RE, Liu J (1997) Tissue polarity points from cells that have higher Frizzled levels towards cells that have lower Frizzled levels. Curr Biol.7:940-949.

Adler PN, Vinson C, Park WJ, Conover S, Klein L (1990) Molecular structure of frizzled, a Drosophila tissue polarity gene. Genetics.,126(2):401-16.

Afzal AR, Rajab A, Fenske CD, Oldridge M, Elanko N, Ternes-Pereira E, Tuysuz B, Murday VA, Patton MA, Wilkie AO, et al. (2000) Recessive Robinow syndrome, allelic to dominant brachydactyly type $B$, is caused by mutation of ROR2. Nat Genet. 25:419-422.

Agapitov AV, Haynes WG (2002) Role of endothelin in cardiovascular disease. J Renin Angiotensin Aldosterone Syst., (1):1-15.

Ahlstrom JD, Erickson CA (2009) The neural crest epithelial-mesenchymal transition in 4D: a 'tail' of multiple non-obligatory cellular mechanisms. Development 136, 18011812.

Alfandari D, Cousin H, Gaultier A, Hoffstrom BG, DeSimone DW (2003) Integrin alpha5beta1 supports the migration of Xenopus cranial neural crest on fibronectin. Developmental Biology 15; 260(2):449-64. 
Alfandari D, Cousin H, Gaultier A, Smith K, White JM, Darribere T, DeSimone DW (2001) Xenopus ADAM 13 is a metalloprotease required for cranial neural crest-cell migration. Curr. Biol. 11, 918-930.

Alfandari D, Cousin H, Marsden M (2010) Mechanism of Xenopus cranial neural crest cell migration. Cell Adh. Migr. 4.

Amit S, Hatzubai A, Birman Y, Andersen JS, Ben-Shushan E, Mann M, Ben-Neriah Y, Alkalay I (2002) Axin-mediated CKI phosphorylation of b-catenin at Ser 45: A molecular switch for the Wnt pathway. Genes Dev 16: 1066-1076.

Anderson RB, Bergner AJ, Taniguchi M, Fujisawa H, Forrai A, Robb L, Young HM (2007) Effects of different regions of the developing gut on the migration of enteric neural crest-derived cells: A role for Sema3A, but not Sema3F. Developmental Biology Vol 305 (1), Pages 287-299.

Anderson RB, Stewart AL, Young HM (2006) Phenotypes of neural-crest-derived cells in vagal and sacral pathways. Cell Tissue Res., 323:11-25.

Aoki Y, Saint-Germain N, Gyda M, Magner-Fink E, Lee YH, Credidio C, SaintJeannet JP (2003) Sox10 regulates the development of neural crest-derived melanocytes in Xenopus. Developmental Biology 259(1): 19-33.

Avilés EC, Wilson NH, Stoeckli ET (2013) Sonic hedgehog and Wnt: antagonists in morphogenesis but collaborators in axon guidance. Front Cell Neurosci. 7:86.

Axelrod JD (2001) Unipolar membrane association of Dishevelled mediates Frizzled planar cell polarity signaling. Genes Dev. 15(10): 1182-1187.

Barlow A, de Graaff E, Pachnis V (2003) Enteric nervous system progenitors are coordinately controlled by the $G$ protein-coupled receptor EDNRB and the receptor tyrosine kinase RET. Neuron 40, 905-916.

Barriga EH, Maxwell PH, Reyes AE, Mayor R (2013). The hypoxia factor Hif-1alpha controls neural crest chemotaxis and epithelial to mesenchymal transition. J. Cell Biol. 201, 759-776.

Barriga EH, Mayor R (2015) Embryonic Cell-Cell Adhesion:A Key Player in Collective Neural Crest Migration. Current Topics in Developmental Biology, Vol 112.

Barrott JJ, Cash GM, Smith AP, Barrow JR, Murtaugh LC (2011) Deletion of mouse Porcn blocks Wnt ligand secretion and reveals an ectodermal etiology of human focal dermal hypoplasia/Goltz syndrome. Proc Natl Acad Sci 108: 12752-12757. 
Barth KA, Kishimoto Y, Rohr KB, Seydler C, Schulte-Merker S ,Wilson SW (1999) Bmp activity establishes a gradient of positional information throughout the entire neural plate. Development 126, 4977-4987.

Basch ML, Bronner-Fraser M, Garcia-Castro MI (2006) Specification of the neural crest occurs during gastrulation and requires Pax7. Nature 441(7090):218-222.

Bastock R., Strutt H., Strutt D. (2003). Strabismus is asymmetrically localised and binds to Prickle and Dishevelled during Drosophila planar polarity patterning. Development 130, 3007-3014.

Baum B, Georgiou M (2011) Dynamics of adherens junctions in epithelial establishment, maintenance, and remodeling. Journal of Cell Biology, 192, 907-917.

Bauma B, Settleman J, Quinlan MP (2008) Transitions between epithelial and mesenchymal states in development and disease. Seminars in Cell \& Developmental Biology 19, 294-308.

Becker S, Mayor R, Kashef J (2013) Cadherin-11 mediates contact inhibition of locomotion during Xenopus neural crest cell migration. PLoS One, 8, e85717.

Behrens J, von Kries JP, Kuhl M, Bruhn L, Wedlich D, Grosschedl R, Birchmeier W (1996) Functional interaction of beta-catenin with the transcription factor LEF-1. Nature 382(6592): 638-42.

Behrens J, von Kries JP, Kuhl M, Bruhn L, Wedlich D, Grosschedl R, Birchmeier W (1996) Functional interaction of beta-catenin with the transcription factor LEF-1. Nature. 382, 638-42.

Bekman E, Henrique D (2002) Embryonic expression of three mouse genes with homology to the Drosophila melanogaster prickle gene. Gene Expr Patterns 2: 73-77.

Belmadani A, Tran PB, Ren D, Assimacopoulos S, Grove EA, Miller RJ (2005) The Chemokine Stromal Cell-Derived Factor-1 Regulates the Migration of Sensory Neuron Progenitors. The Journal of Neuroscience, 25(16): 3995-4003.

Bennett BL, Sasaki DT, Murray BW, O'Leary EC, Sakata ST, Xu W, Leisten JC, Motiwala A, Pierce S, Satoh Y, Bhagwat SS, Manning AM, Anderson DW (2001) SP600125, an anthrapyrazolone inhibitor of Jun N-terminal kinase. Proc. Natl. Acad Sci. USA 98(24):13681-6.

Berndt JD, Clay MR, Langenberg T, Halloran MC (2008) Rho-kinase and myosin II affect dynamic neural crest cell behaviors during epithelial to mesenchymal transition in vivo. Dev. Biol., 324, pp. 236-244. 
Bertsch E, Minturn L, Gotteiner NL, Ernst LM (2015) Absent Aortic Valve in DiGeorge Syndrome. Pediatr. Dev. Pathol.

Biechele S, Cox BJ, Rossant J (2011) Porcupine homolog is required for canonical Wnt signaling and gastrulation in mouse embryos. Dev Biol 355: 275-285.

Bilic J, Huang YL, Davidson G, Zimmermann T, Cruciat CM, Bienz M, Niehrs C (2007) Wnt induces LRP6 signalosomes and promotes disheveled dependent LRP6 phosphorylation. Science 316, 1619-1622.

Bolande RP (1997) Neurocristopathy: its growth and development in 20 years. Pediatr. Pathol. Lab. Med., 17, pp. 1-25.

Bonstein L, Elias S, Frank D. Paraxial-fated mesoderm is required for neural crest induction in Xenopus embryos. Dev. Biol. 1998; 193:156-168.

Borchers A, David R, Wedlich D (2001) Xenopus cadherin-11 restrains cranial neural crest migration and influences neural crest specification. Development, 128 (2001), pp. 3049-3060.

Borday C, Cabochette P, Parain K, Mazurier N, Janssens S, Tran HT, Sekkali B, Bronchain O, Vleminckx K, Locker M, et al., (2012) Antagonistic cross-regulation between Wnt and Hedgehog signalling pathways controls post-embryonic retinal proliferation. Development 139(19): 3499-509.

Bosoi CM, Capra V, Allache R, Trinh VQ, De Marco P, Merello E, et al., (2011) Identification and characterization of novel rare mutations in the planar cell polarity gene PRICKLE1 in human neural tube defects. Human Mutation, 32 pp. 1371-1375

Boutros M, Paricio N, Strutt DI, Mlodzik M (1998) Dishevelled activates JNK and discriminates between JNK pathways in planar polarity and wingless signaling. Cell 94, 109-118.

Bradley LC, Snape A, Bhatt S, Wilkinson DG (1993) The structure and expression of the Xenopus Krox-20 gene: conserved and divergent patterns of expression in rhombomeres and neural crest. Mech. Dev. 40, 73-84.

Brault V, Moore R, Kutsch S, Ishibashi M, Rowitch DH, McMahon AP, Sommer L Boussadia O, Kemler R (2001) Inactivation of the beta-catenin gene by Wnt1Cremediated deletion results in dramatic brain malformation and failure of craniofacial development. Development 128(8): 1253-64.

Bronner-Fraser M, Fraser S (1989) Developmental potential of avian trunk neural crest cells in situ. Neuron Vol. 3, Issue 6, Pages 755-766. 
Burns AJ, Le Douarin NM (1998) The sacral neural crest contributes neurons and glia to the post-umbilical gut: spatiotemporal analysis of the development of the enteric nervous system. Development 125, 4335-4347.

Burridge K, Chrzanowska-Wodnicka M (1996) Focal adhesions, contractility, and signaling. Annu Rev Cell Dev Biol. 12:463-518.

Burridge K, Wennerberg K (2004) Rho and Rac take center stage. Cell 23;116(2):167-79.

Burstyn-Cohen T, Stanleigh J, Sela-Donenfeld D, Kalcheim C (2004) Canonical Wnt activity regulates trunk neural crest delamination linking BMP/noggin signaling with G1/S transition. Development 131(21): 5327-39.

Bustos RI, Forget MA, Settleman JE, Hansen SH (2008). Coordination of Rho and Rac GTPase function via p190B RhoGAP. Curr. Biol. 18, 1606-1611.

Cadigan KM, Waterman ML (2012) TCF/LEFs and Wnt signaling in the nucleus. Cold Spring Harb Perspect Biol.,4(11).

Cai DH, Vollberg Sr TM ,Hahn-Dantona E ,Quigley JP, Brauer PR (2000) MMP-2 expression during early avian cardiac and neural crest morphogenesis. Anat. Rec. 259,168-179.

Cantemir V, Cai DH, Reedy MV, Brauer PR (2004) Tissue inhibitor of metalloproteinase-2 (TIMP-2) expression during cardiac neural crest cell migration and its role in proMMP-2 activation. Dev. Dyn. 231, 709-719.

Capelluto DG, Kutateladze TG, Habas R, Finkielstein CV, He X, Overduin M (2002) The DIX domain targets dishevelled to actin stress fibres and vesicular membranes. Nature 419, 726-729.

Carmen Carrascosa-Romero M, Carlos de Cabo de la Vega (2012) Neurocristopathies: Role of Glial Cells, Genetic Basis and Relevance of Brain Imaging for Diagnosis. Neuroimaging - Clinical Applications. Chapter 5

Carmona-Fontaine C, Mayor R (2008) Directional cell migration in vivo: Wnt at the crest. Cell Adhesion \& Migration, 2, 240-242.

Carreira-Barbosa F, Concha ML, Takeuchi M, Ueno N, Wilson SW, Tada M (2003) Prickle 1 regulates cell movements during gastrulation and neuronal migration in zebrafish. Development 130, 4037- 4046. 
Cast AE, Gao C, Amack JD, Ware SM (2012) An essential and highly conserved role for Zic3 in left-right patterning, gastrulation and convergent extension morphogenesis. Dev Biol., 364(1):22-31.

Cavey M, Lecuit T (2009) Molecular bases of cell-cell junctions stability and dynamics. Cold Spring Harb Perspect Biol 1:a002998.

Cell Adh. Migr. 4.

Chae J, Kim MJ, Goo JH, Collier S, Gubb D, Charlton J, Adler PN, Park WJ (1999) The Drosophila tissue polarity gene starry night encodes a member of the protocadherin family. Development 126(23):5421-9.

Chang C, Hemmati-Brivanlou A (1998) Neural crest induction by Xwnt7B in Xenopus. Dev Biol 194, 129-134.

Chen B, Dodge ME, Tang W, Lu J, Ma Z, Fan C-H, Wei S, Hao W, Kilgore J, Williams NS, Roth MG, Amatruda JF, Chen C, Lum L (2009) Small moleculemediated disruption of Wnt-dependent signaling in tissue regeneration and cancer. Nature Chemical Biology 5, 100 - 107.

Chen G, Courey AJ (2000) Groucho/TLE family proteins and transcriptional repression. Gene., 249(1-2):1-16.

Chen, W.S., D. Antic, M. Matis, C.Y. Logan, M. Povelones, G.A. Anderson, R. Nusse, and J.D. Axelrod (2008) Asymmetric homotypic interactions of the atypical cadherin flamingo mediate intercellular polarity signaling. Cell. 133:1093-1105.

Cheung M, Briscoe J (2003). Neural crest development is regulated by the transcription factor Sox9. Development 130, 5681- 5693.

Cheung M, Chaboissier M-C, Mynett A, Hirst E, Schedl A, Briscoe J (2005) The transcriptional control of trunk neural crest induction, survival, and delamination. Dev. Cell 8, 179-192.

Cheyette BN, Waxman JS, Miller JR, Takemaru K, Sheldahl LC, Khlebtsova N, Fox EP, Earnest T, Moon RT (2002) Dapper, a Dishevelled-associated antagonist of $ß$ catenin and JNK signaling, is required for notochord formation. Dev. Cell 2, 449-461.

Coles EG, Taneyhill LA, Bronner-Fraser M (2007) A critical role for Cadherin6B in regulating avian neural crest emigration. Dev. Biol. 312, 533-544.

Cordero DR, Brugmann S, Chu Y, Bajpai R, Jame M, Helms JA (2011) Cranial Neural Crest Cells On The Move: Their Roles In Craniofacial Development. Am. J. Med. Genet. A.,1,155(2): 270-279. 
Coudreuse D, Korswagen HC (2007) The making of Wnt: new insights into Wnt maturation, sorting and secretion. Development.,134(1):3-12.

Culotti JG, Merz DC (1998) DCC and netrins. Curr Opin Cell Biol. 5, 609-13.

Dajani R, Faser E, Roe SM, Yeo M, Good VM, Thompson V, Dale TC, Pearl LH (2003) Structural basis for recruitment of glycogen synthase kinase $3 b$ to the axinAPC scaffold complex. EMBO J 22: 494-501.

Daniels DL Weis WI (2005) Beta-catenin directly displaces Groucho/TLE repressors from Tcf/Lef in Wnt-mediated transcription activation. Nat Struct Mol Biol., 12(4):36471

Darken RS, Scola AM, Rakeman AS, Das G, Mlodzik M, Wilson PA (2002) The planar polarity gene strabismus regulates convergent extension movements in Xenopus. EMBO J. 21:976-985.

Davidson G, Wu W, Shen J, Bilic J, Fenger U, Stannek P, Glinka A, Niehrs C (2005) Casein kinase 1 gamma couples Wnt receptor activation to cytoplasmic signal transduction. Nature. 438, 867-72.

De Bellard ME, Rao Y, Bronner-Fraser M (2003) Dual function of Slit2 in repulsion and enhanced migration of trunk, but not vagal, neural crest cells. J. Cell Biol. 162, 269-279.

De Calisto J , Araya C , Marchant L , Riaz CF , Mayor R (2005) Essential role of noncanonical Wnt signalling in neural crest migration. Development 132 (11): 2587-97.

de Melker AA, Desban N, Duband JL (2004) Cellular localization and signaling activity of beta-catenin in migrating neural crest cells. Developmental dynamics: an official publication of the American Association of Anatomists 230(4): 708-26.

De Marco P, Merello E, Rossi A, Piatelli G, Cama A, Kibar Z et al., (2012) FZD6 is a novel gene for human neural tube defects. Human Mutation, 33 pp. 384-390

Deardorff MA, Tan C, Saint-Jeannet JP, Klein PS (2001) A role for frizzled 3 in neural crest development. Development 128, 3655-3663.

DeChiara TM, Kimble RB, Poueymirou WT, Rojas J, Masiakowski P, Valenzuela DM, Yancopoulos GD (2000) Ror2, encoding a receptor-like tyrosine kinase, is required for cartilage and growth plate development. Nat Genet. 24:271-274.

Dee CT, Szymoniuk CR, Mills PE, Takahashi T (2013) Defective neural crest migration revealed by a Zebrafish model of Alx1-related frontonasal dysplasia. Hum. Mol. Genet., 15; 22(2):239-51. 
DeLuca SM, Gerhart J, Cochran E, Simak E, Blitz J, Mattiacci-Paessler M, Knudsen K, George-Weinstein M (1999) Hepatocyte growth factor/scatter factor promotes a switch from E- to N-cadherin in chick embryo epiblast cells. Exp. Cell Res. 251, 3-15.

Devenport D (2014) The cell biology of planar cell polarity. JCB vol. 207 no. 2 171179.

Dickinson ME, Selleck MA, McMahon AP, Bronner-Fraser M. Dorsalization of the neural tube by the non-neural ectoderm. Development 1995;121:2099-2106.

Dorsky RI, Moon RT, Raible DW (1998) Control of neural crest cell fate by the Wnt signalling pathway. Nature 396(6709): 370-3.

Dottori M, Gross MK, Labosky P, Goulding M (2001) The winged-helix transcription factor Foxd3 suppresses interneuron differentiation and promotes neural crest cell fate. Development 128, 4127-4138.

Duban JL (2010) Diversity in the molecular and cellular strategies of epithelium-tomesenchyme transitions Insights from the neural crest. Cell Adhesion \& Migration $4: 3,458-482$.

Duband JL (2010) Diversity in the molecular and cellular strategies of epithelium-tomesenchyme transitions: insights from the neural crest. Cell Adh. Migr. 4, pp. 458482

Duong TD, Erickson CA (2004) MMP-2 plays an essential role in producing epithelial-mesenchymal transformations in the avian embryo Dev. Dyn. 229, 42-53.

Dupin E, Creuzet S, Le Douarin NM (2006) The contribution of the neural crest to the vertebrate body. Adv. Exp. Med. Biol., 589, pp. 96-119.

Dupin E, Sommer L (2012) Neural crest progenitors and stem cells: From early development to adulthood. Dev. Biol., 366, 83-95.

Ennis BW, Fultz KE, Smith KA, Westwick JK, Zhu D, Boluro-Ajayi M, Bilter GK, Stein B (2004) Inhibition of Tumor Growth, Angiogenesis, and Tumor Cell Proliferation by a Small Molecule Inhibitor of c-Jun N-terminal Kinase. The Journal of Pharmacology and Experimental Therapeutics 313:325-332.

Fairchild CL, Gammill LS (2013) Tetraspanin18 is a FoxD3-responsive antagonist of cranial neural crest epithelial-to-mesenchymal transition that maintains cadherin-6B protein. J. Cell Sci. 126, 1464-1476. 
Faure S, de Santa Barbara P, Roberts DJ, Whitman M (2002) Endogenous patterns of BMP signaling during early chick development. Dev Biol 244(1):44-65.

Feiguin F, Hannus M, Mlodzik M, Eaton S (2001) The ankyrin repeat protein Diego mediates Frizzled-dependent planar polarization. Dev. Cell 1, 93-101.

Ferronha T, Rabadan MA, Gil-Guinon E, Le Dreau G, de Torres C, Marti E (2013). LMO4 is an essential cofactor in the Snail2-mediated epithelialto- mesenchymal transition of neuroblastoma and neural crest cells. J. Neurosci. 33, 2773-2783.

Gal N, Lechtman-Goldstein D, Weihs D (2013) Particle tracking in living cells: a review of the mean square displacement method and beyond. Rheologica Acta 52(5): 425-443.

Gammill LS, Gonzalez C, Gu C, Bronner-Fraser M (2006) Guidance of trunk neural crest migration requires neuropilin 2/semaphorin 3F signaling. Development 133, 99106.

Gao (2012) Wnt regulation of Planar Cell Polarity (PCP) Current Topics in Developmental Biology Vol 101.

Gao, B., H. Song, K. Bishop, G. Elliot, L. Garrett, M.A. English, P. Andre, J. Robinson, R. Sood, Y. Minami, et al (2011) Wnt signaling gradients establish planar cell polarity by inducing Vangl2 phosphorylation through Ror2. Dev. Cell. 20:163-176.

García-Castro MI, Marcelle C, Bronner-Fraser M (2002) Ectodermal Wnt function as a neural crest inducer. Science 297, 848-851.

Garriock RJ, Krieg PA (2006) Wnt11-R signaling regulates a calcium sensitive EMT event essential for dorsal fin development of Xenopus. Dev. Biol. 304, 127-140.

Gaultier A, Cousin H, Darribere T, Alfandari D (2002) ADAM13 disintegrin and cysteine-rich domains bind to the second heparin-binding domain of fibronectin. $\mathrm{J}$. Biol. Chem. 277, 23 336-23 344.

Ghogomu SM, van Venrooy S, Ritthaler M, Wedlich D, Gradl D (2006) HIC-5 is a novel repressor of lymphoid enhancer factor/T-cell factor-driven transcription. J Biol

Goodrich LV, Strutt D (2011) Principles of planar polarity in animal development. Development, 138 pp. 1877-1892

Goto T, Keller R (2002) The planar cell polarity gene Strabismus regulates convergence and extension and neural fold closure in Xenopus. Dev. Biol. 247:165181. 
Graham A, Begbie J, McGonnell I (2004) Significance of the Cranial Neural Crest. Developmental Dynamics 229:5-13.

Gray RS, Roszko I, Solnica-Krezel L (2011) Planar cell polarity: Coordinating morphogenetic cell behaviors with embryonic polarity. Developmental Cell, 21 pp. 120-133

Green JL, Kuntz SG, Sternberg PW (2008) Ror receptor tyrosine kinases: Orphans no more. Trends Cell Biol. 18:536-544.

Grenier J, Teillet MA, Grifone R, Kelly RG, Duprez D (2009) Relationship between neural crest cells and cranial mesoderm during head muscle development PLoS One, 4, p. e4381.

Gros J, Serralbo O, Marcelle C (2008) WNT11 acts as a directional cue to organize the elongation of early muscle fibres. Nature 457:589-593.

Grumolato L, Liu G, Haremaki T, Mungamuri SK, Mong P, Akiri G, Lopez-Bergami P, Arita A, Anouar Y, Mlodzik M, Ronai ZA, Brody J, Weinstein DC, Aaronson SA (2013) $\beta$-Catenin-independent activation of TCF1/LEF1 in human hematopoietic tumor cells through interaction with ATF2 transcription factors. LoS Genet.,9(8):e1003603

Gubb D, Green C, Huen D, Coulson D, Johnson G, Tree D, Collier S, Roote J (1999) The balance between isoforms of the prickle LIM domain protein is critical for planar polarity in Drosophila imaginal discs. Genes Dev. 13, 2315-2327.

Ha N-C, Tonozuka T, Stamos JL, Choi H-J, Weis WI (2004) Mechanism of phosphorylation-dependent binding of APC to ß-catenin and its role in ß-catenin degradation. Mol Cell 15: 511-521.

Habas R, Kato Y, He X (2001) Wnt/Frizzled activation of Rho regulates vertebrate gastrulation and requires a novel Formin homology protein Daam1. Cell., 107(7):84354

Halbleib JM, Nelson WJ (2006) Cadherins in development: cell adhesion, sorting, and tissue morphogenesis. Genes Dev. 20, 3199-3214.

Hall B (2008) The neural crest and neural crest cells in vertebrate development and evolution. Springer, New York.

Hammerschmidt M, Pelegri F, Mullins MC, Kane DA, Brand M, van Eeden FJM, Furutani-Seiki M, Granato M, Haffter P, Heisenberg C-P, Jiang Y-J, Kelsh RN, Odenthal J, Warga RM, Nüsslein-Volhard C (1996) Mutations affecting morphogenesis during gastrulation and tail formation in the zebrafish, Danio rerio. Development 123, 143-151. 
Han Z, Boyle DL, Chang L, Bennett B, Karin M, Yang L, Manning AM, Firestein GS (2001) C-Jun N-terminal kinase is required for metalloproteinase expression and joint destruction in inflammatory arthritis. J Clin Invest. 108(1):73-81.

Hari L, Brault V, Kleber M, Lee HY, Ille F, Leimeroth R, Paratore C, Suter U, Kemler $R$, Sommer $L$ (2002) Lineage-specific requirements of beta-catenin in neural crest development. The Journal of cell biology 159(5): 867-80.

Hari L, Miescher I, Shakhova O, Suter U, Chin L, Taketo M, Richardson WD Kessaris $\mathrm{N}$, Sommer L (2012) Temporal control of neural crest lineage generation by Wnt/beta-catenin signaling. Development 139(12): 2107-17.

Harland RM (1991) In situ hybridization: an improved whole-mount method for Xenopus embryos. Methods Cell Biol 36: 685-95.

Harrison M, Abu-Elmagd M, Grocott T, Yates C, Gavrilovic J, Wheeler GN (2004) Matrix metalloproteinase genes in Xenopus development. Dev. Dyn. 231, 214-220.

Hart MJ, de los Santos R, Albert IN, Rubinfeld B, Polakis P (1998) Downregulation of B-catenin by human Axin and its association with the APC tumor suppressor, $B$ catenin and GSK3ß. Curr Biol 8: 573-581.

Hassler C, Cruciat CM, Huang YL, Kuriyama S, Mayor R, Niehrs C (2007) Kremen is required for neural crest induction in Xenopus and promotes LRP6-mediated Wnt signaling. Development 134(23):4255-4263.

He X, Semenov M, Tamai K, Zeng X (2004) LDL receptorrelated proteins 5 and 6 in Wnt/b-catenin signaling: Arrows point the way. Development 131: 1663-1677.

Heanue TA, Pachnis V (2007) Enteric nervous system development and Hirschsprung's disease: advances in genetic and stem cell studies. Nat Rev Neurosci., 8(6):466-79.

Heckman CA (2009) Contact inhibition revisited. Journal of Cellular Physiology, 220, 574-575.

Heisenberg C.P., M. Tada, G.J. Rauch, L. Saúde, M.L. Concha, R. Geisler, D.L. Stemple, J.C. Smith, and S.W. Wilson (2000) Silberblick/Wnt11 mediates convergent extension movements during zebrafish gastrulation. Nature 405:76-81.

Helbling PM, Tran CT, Brandli AW (1998) Requirement for EphA receptor signaling in the segregation of Xenopus third and fourth arch neural crest cells. Mech. Dev. 78, 63-79. 
Higgs, HN (2005) Formin proteins: a domain-based approach. Trends Biochem.Sci. 30, 342-353.

Hikasa H, Shibata M, Hiratani I, Taira M (2002) The Xenopus receptor tyrosine kinase Xror2 modulates morphogenetic movements of the axial mesoderm and neuroectoderm via Wnt signaling. Development 1.129 (22): 5227-39.

Himanen JP et al. 2004 Repelling class discrimination: ephrin-A5 binds to and activates EphB2 receptor signaling. Nat. Neurosci. 7, 501-509.

Hinck L, Nelson WJ, Papkoff J (1994) Wnt-1 modulates cell-cell adhesion in mammalian cells by stabilizing beta-catenin binding to the cell adhesion protein cadherin. J Cell Biol.,124(5):729-41.

Hofmann K (2000) A superfamily of membrane-bound Oacyltransferases with implications for Wnt signaling. Trends Biochem Sci 25: 111-112.

Hong CS, Park BY, Saint-Jeannet JP (2008) Fgf8a induces neural crest indirectly through the activation of Wnt8 in the paraxial mesoderm. Development 135(23):39033910.

Hopwood ND, Pluck A, Gurdon JB (1989) A Xenopus mRNA related to Drosophila twist is expressed in response to induction in the mesoderm and the neural crest. Cell 59(5):893-903.

Hsu W, Zeng L, Costantini $F$ (1999) Identification of a domain of Axin that binds to the serine/threonine protein phosphatase $2 \mathrm{~A}$ and a self-binding domain. $\mathrm{J}$ Biol Chem 274: 3439-3445.

Huang C, Rajfur Z, Borchers C, Schaller MD, Jacobson K (2003) JNK phosphorylates paxillin and regulates cell migration. Nature $\mathrm{Vol} 424$

Huang GY, Wessels A, Smith BR, Linask KK, Ewart JL, Lo CW (1998) Alteration in Connexin 43 Gap Junction Gene Dosage Impairs Conotruncal Heart Development. Developmental Biology 198, 32-44.

Ikeda S, Kishida S, Yamamoto H, Murai H, Koyama S, Kikuchi A (1998) Axin, a negative regulator of the Wnt signaling pathway, forms a complex with GSK-3b and beta-catenin and promotes GSK-3beta-dependent phosphorylation of beta-catenin. EMBO J.,17(5)

Ikenouchi J, Matsuda M, Furuse M, Tsukita S (2003) Regulation of tight junctions during the epithelium-mesenchyme transition: direct repression of the gene expression of claudins/occludin by Snail. Journal of Cell Science 116, 1959-1967. 
Itoh RE, Kurokawa K, Ohba Y, Yoshizaki H, Mochizuki N, Matsuda M (2002). Activation of Rac and Cdc42 video-imaged by FRET-based single-molecule probes in the membrane of living cells. Mol. Cell. Biol. 22:6582-6591.

Janda CY, Waghray D, Levin AM, Thomas C, Garcia KC (2012) Structural basis of Wnt recognition by Frizzled. Science doi: 10.1126/science.1222879.

Jenei V, Sherwood V, Howlin J, Linnskog R, Säfholm A, Axelsson L, Andersson T (2009) A t-butyloxycarbonyl-modified Wnt5a-derived hexapeptide functions as a potent antagonist of Wnt5a-dependent melanoma cell invasion. PNAS Vol.106

Jessen JR, Topczewski J, Bingham S, Sepich DS, Marlow F, Chandrasekhar A, Solnica-Krezel L (2002) Zebrafish trilobite identifies new roles for Strabismus in gastrulation and neuronal movements. Nat. Cell Biol. 4:610-615.

Jia L, Cheng L, Raper J (2005) Slit/Robo signaling is necessary to confine early neural crest cells to the ventral migratory pathway in the trunk. Dev. Biol. 282, 411421.

Jiang Y, Liu M, Gershon MD (2003) Netrins and DCC in the guidance of migrating neural crest-derived cells in the developing bowel and pancreas. Developmental Biology Vol 258 (2), Pages 364-384.

Joosa TO, Whittakerb CA, Mengb F, DeSimoneb DW, Gnauc V, Hausen P (1995) Integrin a5 during early development of Xenopus laevis. Mechanisms of Development 50, 187- 199.

Joseph MJ, Dangi-Garimella S, Shields MA, Diamond ME, Sun L, Koblinski JE, Munshi HG (2009) Slug Is a Downstream Mediator of Transforming Growth Factorb1-Induced Matrix Metalloproteinase-9 Expression and Invasion of Oral Cancer Cells. Journal of Cellular Biochemistry 108:726-736.

Jung B , Köhler A , Schambony A , Wedlich D (2011) PAPC and the Wnt5a/Ror2 pathway control the invagination of the otic placode in Xenopus. BMC Dev Biol. 11 36.

Kadowaki T,Wilder E, Klingensmith J, Zachary K, Perrimon N (1996) The segment polarity gene porcupine encodes a putative multitransmembrane protein involved inWingless processing. Genes Dev 10: 3116-3128.

Kanesaki T, Edwards CM, Schwarz US, Grosshans J (2011) Dynamic ordering of nuclei in syncytial embryos: a quantitative analysis of the role of cytoskeletal networks. Integrative biology: quantitative biosciences from nano to macro 3(11): 1112-9. 
Karner $\mathrm{CM}$, Chirumamilla R, Aoki $\mathrm{S}$, et al. Wnt9b signaling regulates planar cell polarity and kidney tubule morphogenesis. Nat Genet. 2009; 41:793-799.

Kasemeier-Kulesa JC, Bradley R, Pasquale EB, Lefcort F, Kulesa PM (2006) Eph/ephrins and $\mathrm{N}$-cadherin coordinate to control the pattern of sympathetic ganglia. Development 133, 4839-4847.

Kasemeier-Kulesa JC, McLennan R, Romine MH, Kulesa PM, Lefcort F (2010) CXCR4 controls ventral migration of sympathetic precursor cells. J. Neurosci. 0:13078-13088.

Kashef J, Köhler A, Kuriyama S, Alfandari D, Mayor R, Wedlich D (2009) Cadherin11 regulates protrusive activity in Xenopus cranial neural crest cells upstream of Trio and the small GTPases. Genes \& Development 23:1393-1398.

Keyte A, Hutson MR (2012) The neural crest in cardiac congenitala nomalies. Differentiation 84, 25-40.

Kibar Z, Bosoi CM, Kooistra M, Salem S, Finnell RH, De Marco P, et al., (2009) Novel mutations in VANGL1 in neural tube defects. Human Mutation, 30 pp. E706E715

Kibar Z, Capra V, Gros P (2007) Toward understanding the genetic basis of neural tube defects. Clinical Genetics, 71 pp. 295-310

Kikuchi A, Yamamoto H, Sato A, Matsumoto S (2012) Wnt5a: its signalling, functions and implication in diseases. Acta Physiol (Oxf)., 204(1):17-33.

Kil SH, Lallier T, Bronner-Fraser M (1996) Inhibition of Cranial Neural Crest Adhesion in Vitro and Migration in Vivo Using Integrin Antisense Oligonucleotides. Developmental Biology 179, 91-101

Kim G-H, Her J-H, Han J-K (2008) Ryk cooperates with Frizzled 7 to promote Wnt11mediated endocytosis and is essential for Xenopus laevis convergent extension movements. J. Cell Biol. 182:1073-1082.

Kirby ML, Gale TF, Stewart DE (1983). Neural crest cells contribute to normal aorticopulmonary septation. Science 220, 1059-1061.

Kirby ML, Hutson MR (2010) Factors controlling cardiac neural crest cell migration Kirby ML, Waldo KL (1995). Neural crest and cardiovascular patterning. Circ. Res. 77, 211-215. 
Kitagawa M, Hatakeyama S, Shirane M, Matsumoto M, Ishida N, Hattori K, Nakmichi I, Kikuchi A, Nakayama K, Nakayama K (1999) An F-box protein, FWD1, mediates ubiquitin-dependent proteolysis of beta-catenin. EMBO J.,18(9):2401-10

Klein PS, Melton DA (1996) A molecular mechanism for the effect of lithium on development. Proc. Natl. Acad. Sci. USA. 93:8455-8459.

Klein TJ, Mlodzik M (2005) Planar cell polarization: an emerging model points in the right direction. Annu Rev Cell Dev Biol. 21:155-76.

Knöchel S, Schuler-Metz A, Knöchel W (2000) c-Jun (AP-1) activates BMP-4 transcription in Xenopus embryos. Mech Dev., 98(1-2):29-36.

Koblar SA, Krull CE, Pasquale EB, McLennan R, Peale FD, Cerretti DP, Bothwell M (2000) Spinal motor axons and neural crest cells use differentmolecular guides for segmental migration through the rostral half-somite. J. Neurobiol. 42, 437-447.

Kohn AD, Moon RT (2005) Wnt and calcium signaling: beta-catenin-independent pathways. Cell Calcium., 38(3-4):439-46.

Kolodkin AL (1998) Semaphorin-mediated neuronal growth cone guidance. Prog. Brain Res. 117, 115-132.

Komekado H, Yamamoto H, Chiba T, Kikuchi A (2007) Glycosylation and palmitoylation of Wnt-3a are coupled to produce an active form of Wnt-3a. Genes Cells 12:521-534

Komiya Y, Habas R (2008) Wnt signal transduction pathways. Organogenesis, 4(2): 68-75.

Krull CE, Lansford R, Gale NW, Collazo A, Marcelle C, Yancopoulos GD, Fraser SE, Bronner-Fraser M (1997) Interactions of Eph-related receptors and ligands confer rostrocaudal pattern to trunk neural crest migration. Curr. Biol. 7, 571-580.

Kuo BR, Erickson CA (2011) Vagal neural crest cell migratory behavior: A transition between the cranial and trunk crest. Developmental Dynamics Vol. 240 (9) 2084 2100.

Kurayoshi M, Yamamoto H, Izumi S, Kikuchi A (2007) Posttranslational palmitoylation and glycosylation of Wnt-5a are necessary for its signalling. Biochem J 402: 515-523.

Kuriyama S, Mayor R (2008) Molecular analysis of neural crest migration. Philos. 
Kuriyama S, Theveneau E, Benedetto A, Parsons M, Tanaka M, Charras G, Kabla A, Mayor R (2014) In vivo collective cell migration requires an LPAR2-dependent increase in tissue fluidity. J. Cell Biol. Vol. 206 No. 1 113-127.

LaBonne C, Bronner-Fraser M (1998) Neural crest induction in Xenopus: evidence for a two-signal model. Development 125, 2403-2414.

Lallier TE, Bronner-Fraser M (1991) Avian neural crest cell attachment to laminin: involvement of divalent cation dependent and independent integrins. Development 113, 1069-1084.

Lallier TE, Whittaker CA, DeSimone DW (1996) Integrin a6 expression is required for early nervous system development in Xenopus laevis. Development 122, 2539-2554.

Lander R, Nasr T, Ochoa SD, Nordin K, Prasad MS, Labonne C (2013) Interactions between Twist and other core epithelial-mesenchymal transition factors are controlled by GSK3-mediated phosphorylation. Nature communications 4: 1542.

Latres E, Chiaur DS, Pagano M (1999) Human F box protein ß-Trcp associates with the Cul1/Skp1 complex and regulates the stability of b-catenin. Oncogene 18: 849854.

Le Douarin N, Kalcheim C (1999) The neural crest. Cambridge University Press, Cambridge, UK; New York, NY, USA.

Le Douarin N, Teillet MA (1971) Localization, by the method of interspecific grafts of the neural area from which adrenal cells arise in the bird embryo. C. R. Acad. Sci. Hebd. Seances Acad. Sci. D, 272, pp. 481- 484.

Lee HO, Levorse JM, Shin MK (2003) The endothelin receptor-B is required for the migration of neural crest-derived melanocyte and enteric neuron precursors. Dev. Biol. 259, 162-175.

Lee HY, Kleber M, Hari L, Brault V, Suter U, Taketo MM, Kemler R, Sommer L (2004) Instructive role of $\mathrm{Wnt} /$ beta-catenin in sensory fate specification in neural crest stem cells. Science 303(5660): 1020-3.

Leibl MA et al. (1999) Expression of endothelin 3 by mesenchymal cells of embryonic mouse caecum. Gut 44, 246-252.

Lewis AE, Vasudevan HN, O'Neill AK, Soriano P, Bush JO (2013) The widely used Wnt1-Cre transgene causes developmental phenotypes by ectopic activation of Wnt signaling. Developmental Biology 379(2): 229-34. 
Lewis JL, Bonner J, Modrell M, Ragland JW, Moon RT, Dorsky RI, Raible DW (2004) Reiterated Wnt signaling during zebrafish neural crest development. Development 131(6): 1299-308.

Li Z, Dong X, Wang Z, Liu W, Deng N, Ding Y, Tang L, Hla T, Zeng R, Li L, Wu D (2005) Regulation of PTEN by Rho small GTPases. Nat Cell Biol. 7(4):399-404.

Li Z, Nie F, Wang S, Li L (2011) Histone H4 Lys 20 monomethylation by histone methylase SET8 mediates Wnt target gene activation. Proc Natl Acad Sci 108: 31163123

Liu C, Kato Y, Zhang Z, Do VM, Yankner BA, He X (1999) B-Trcp couples b-catenin phosphorylation-degradation and regulates Xenopus axis formation. Proc Natl Acad Sci.,96: 6273-6278.

Liu C, Li Y, Semenov M, Han C, Baeg G-H, Tan Y, Zhang Z, Lin X, He X (2002) Control of $ß$-catenin phosphorylation/ degradation by a dual-kinase mechanism. Cell 108: 837-847.

Liu JAJ, Wu M-H, Yan CH, Chau BKH, So H, Ng A, Chan A, Cheah KSE, Briscoe J, Cheung M (2013) Phosphorylation of Sox9 is required for neural crest delamination and is regulated downstream of BMP and canonical Wnt signaling. Proc. Natl. Acad. Sci. USA 110, 2882-2887.

Liu W, Sato A, Khadka D, Bharti R, Diaz H, Runnels LW, Habas R (2008) Mechanism of activation of the Formin protein Daam1. PNAS Vol. 105.

Logan CY, Nusse R (2004) The Wnt signaling pathway in development and disease. Annu Rev Cell Dev Biol.,20:781-810.

Lu X, Borchers AG, Jolicoeur C, Rayburn H, Baker JC, Tessier-Lavigne M (2004) PTK7/CCK-4 is a novel regulator of planar cell polarity in vertebrates. Nature 430:938.

Lu X, Borchers AG, Jolicoeur C, Rayburn H, Baker JC, Tessier-Lavigne M (2004) PTK7/CCK-4 is a novel regulator of planar cell polarity in vertebrates. Nature, 430, 93-98.

Lyons JP, Miller RK, Zhou X, Weidinger G, Deroo T, Denayer T, Park JI, Ji H, Hong $\mathrm{JY}$, Li A, et al., (2009) Requirement of Wnt/beta-catenin signaling in pronephric kidney development. Mechanisms of development 126(3-4): 142-59. 
MacDonald BT, He X (2012) Frizzled and LRP5/6 receptors for Wnt/3-catenin signaling. Cold Spring Harb Perspect Biol., 4(12).

Macheda ML, Sun WW, Kugathasan K, Hogan BM, Bower NI, Halford MM, Zhang YF, Jacques BE, Lieschke GJ, Dabdoub A, Stacker SA (2012) The Wnt receptor Ryk plays a role in mammalian planar cell polarity signaling. J. Biol. Chem. 287, 2931229323.

Mahmoudi A, Rami M, Khattala K, Elmadi A, Afifi MA, Youssef B (2013) ShahWaardenburg syndrome. Pan. Afr. Med. J.14:60.

Mancilla A, Mayor R. Neural crest formation in Xenopus laevis: mechanisms of Xslug induction. Dev. Biol. 1996; 177:580-589.

Marchant L, Linker C, Ruiz P, Guerrero N, Mayor R. The inductive properties of mesoderm suggest that the neural crest cells are specified by a BMP gradient. Dev Biol 1998; 198:319-329.

Mason I (2007) Initiation to end point: the multiple roles of fibroblast growth factors in neural development. Nat Rev Neurosci.,583-96.

Matusek T., Gombos R., Szecsenyi A., Sanchez-Soriano N., Czibula A., Pataki C., Gedai A., Prokop A., Rasko I., Mihaly J. (2008). Formin proteins of the DAAM subfamily play a role during axon growth. J. Neurosci. 28, 13310-13319.

Mayor R, Morgan R, Sargent MG. Induction of the prospective neural crest of Xenopus. Development 1995; 121:767-777.

Mayor R, Young R, Vargas A (1999) Development of neural crest in Xenopus. Current topics in Developmental Biology, 43 85-113.

McCusker C, Cousin H, Neuner R, Alfandari D (2009) Extracellular cleavage of cadherin-11 by ADAM metalloproteases is essential for Xenopus cranial neural crest cell migration. Mol Biol Cell 20:78-89.

McLennan R, Krull CE (2002) Ephrin-as cooperate with EphA4 to promote trunk neural crest migration. Gene. Expr. 10, 295-305.

Meijer L, Skaltsounis AL, Magiatis P, Polychronopoulos P, Knockaert M, Leost M, Ryan XP, Vonica CA, Brivanlou A, Dajani R, et al., (2003) GSK-3-selective inhibitors derived from Tyrian purple indirubins. Chemistry \& biology 10(12): 1255-66.

Mellitzer G, Xu Q, Wilkinson DG (1999) Eph receptors and ephrins restrict cell intermingling and communication. Nature 400, 77-81. 
Meng W, Takeichi M (2009) Adherens junction: Molecular architecture and regulation. Cold Spring Harb Perspect Biol 1:a002899.

Merrill AE, Bochukova EG, Brugger SM, Ishii M, Pilz DT, Wall SA, Lyons KM, Wilkie AO, Maxson Jr RE (2006) Cell mixing at a neural crest-mesodermboundary and deficient ephrin-Eph signaling in the pathogenesis of craniosynostosis. Hum. Mol. Genet. 15, 1319-1328.

Mikels AJ, Nusse R (2006) Purified Wnt5a protein activates or inhibits beta-cateninTCF signaling depending on receptor context. PLoS Biology, 4, e115.

Miller JR, Hocking AM, Brown JD, Moon RT (1999) Mechanism and function of signal transduction by the Wnt/b-catenin and Wnt/Ca2+ pathways. Oncogen, 18, 78607872.

Minami Y, Oishi I, Endo M, Nishita M (2009) Ror-family receptor tyrosine kinases in noncanonical Wnt signaling: Their implications in developmental morphogenesis and human diseases. Developmental Dynamics Vol 239, Issue 1, pages 1-15.

Minoux M, Rijli FM (2010) Molecular mechanisms of cranial neural crest cell migration and patterning in craniofacial development. Development, 137, pp. 26052621.

Molenaar M , Roose J , Peterson J , Venanzi S , Clevers H, Destrée O (1998) Differential expression of the HMG box transcription factors XTcf-3 and XLef-1 during early xenopus development. Mech Dev.75 (1-2): 151-4.

Molenaar M, van de Wetering M, Oosterwegel M, Peterson-Maduro J, Godsave S, Korinek V, Roose J, Destree O, Clevers H (1996) XTcf-3 transcription factor mediates beta-catenin-induced axis formation in Xenopus embryos. Cell. 86, 391-9.

Montcouquiol M, Crenshaw EB 3rd, Kelley MW (2006) Noncanonical Wnt signaling and neural polarity. Annu Rev Neurosci., 29:363-86.

Moore AC, Amann JM,Williams CS, Tahinci E, Farmer TE, Martinez JA, Yang G, Luce KS, Lee E, Hiebert SW (2008) Myeloid translocation gene family members associate with T-cell factors (TCFs) and influence TCF-dependent transcription. Mol Cell Biol 28: 977-987.

Morales AV, Barbas JA, Nieto MA (2005) How to become neural crest: from segregation to delamination. Semin. Cell Dev. Biol. 16, 655-662. 
Morgan R, Sargent MG (1997) The role in neural patterning of translation initiation factor elF4All; induction of neural fold genes. Development 124:2751-2760.

Moriguchi T, Kawachi K, Kamakura S, Masuyama N, Yamanaka H, Matsumoto K, Kikuchi A, Nishida E (1999) Distinct domains of mouse dishevelled are responsible for the c-Jun N-terminal kinase/stressactivated protein kinase activation and the axis formation in vertebrates. J. Biol. Chem. 274, 30957-30962.

Moriyoshi K, Richards LJ, Akazawa C, O'Leary DD, Nakanishi S (1996) Labeling neural cells using adenoviral gene transfer of membrane-targeted GFP Neuron, 16, pp. 255-260.

Moury D, Jacobson AG (1989) Neural fold formation at newly created boundaries between neural plate and epidermis in the axolotl. Developmental Biology 133, 4457.

Mullis K, Faloona F, Scharf S, Saiki R, Horn G, Erlich H (1986) Specific enzymatic amplification of DNA in vitro: the polymerase chain reaction. Cold Spring Harb. Symp. Quant. Biol. 51 Pt 1: 263-273.

Myers CT, Appleby SC, Krieg PA (2014) Use of small molecule inhibitors of the Wnt and Notch signaling pathways during Xenopus development. Methods 66(3): 380-9.

Nakaya MA, Habas R, Biris K, Dunty WC, Jr Kato Y, He X, Yamaguchi TP (2004) Identification and comparative expression analyses of Daam genes in mouse and Xenopus. Gene Expr. Patterns 5, 97-105.

Natarajan D, Marcos-Gutierrez C, Pachnis V, de Graaff E (2002) Requirement of signalling by receptor tyrosine kinase RET for the directed migration of enteric nervous system progenitor cells during mammalian embryogenesis. Development 129, 5151-5160.

Nguyen VH, Schmid B, Trout J, Connors SA, Ekker M, Mullins MC. Ventral and lateral regions of the zebrafish gastrula, including the neural crest progenitors, are established by a bmp2b/swirl pathway of genes. Dev Biol 1998; 199:93-110.

Nieto A (2011) The Ins and Outs of the Epithelial to Mesenchymal Transition in Health and Disease. Annu. Rev. Cell Dev. Biol. 27:347-76.

Nieto A, Sargent MG, Wilkinson DG, Cooke J (1994) Control of Cell Behavior During Vertebrate Development by Slug, a Zinc Finger Gene. Science Vol. 264.

Nieto MA, Sargent MG, Wilkinson DG, Cooke J (1994) Control of cell behavior during vertebrate development by Slug, a zinc finger gene. Science 264:835-839.

Noden D.M (1988) Interactions and fates of avian craniofacial mesenchyme. Craniofacial Development. Development 103. 
Noden DM (1975) An analysis of the migratory behavior of avian cephalic neural crest cells. Developmental Biology Vol. 42 (1), 106-13

Oinuma I, Katoh H, Negishi M (2006) Semaphorin 4D/Plexin-B1-mediated R-Ras GAP activity inhibits cell migration by regulating $\beta_{1}$ integrin activity. JCB vol. 173 no. 4 601-613.

Oishi I, Suzuki H, Onishi N, Takada R, Kani S, Ohkawara B, Koshida I, Suzuki K, Yamada G, Schwabe GC, et al. (2003) The receptor tyrosine kinase Ror2 is involved in non-canonical Wnt5a/JNK signalling pathway. Genes Cells 8:645-654.

Orford K, Crockett C, Jensen JP, Weissman AM, Byers SW (1997) Serine phosphorylation-regulated ubiquitination and degradation of ß-catenin. J Biol Chem 272: 24735-24738.

Osborne NJ, Begbie J, Chilton JK, Schmidt H, Eickholt BJ (2005) Semaphorin/neuropilin signaling influences the positioning of migratory neural crest cells within the hindbrain region of the chick. Dev. Dyn. 232, 939-949.

Pandur P, Läsche M, Eisenberg LM, Kühl M (2002) Wnt-11 activation of a noncanonical Wnt signalling pathway is required for cardiogenesis. Nature, 418(6898):636-41.

Park WJ, Liu J, Adler PN (1994) The frizzled gene of Drosophila encodes a membrane protein with an odd number of transmembrane domains. Mech Dev., 45(2):127-13.

Patthey C, Edlund T, Gunhaga L (2009) Wnt-regulated temporal control of BMP exposure directs the choice between neural plate border and epidermal fate. Development 136(1):73-83.

Penton, A., Wodarz, A. and Nusse, R. (2002). A mutational analysis of dishevelled in Drosophila defines novel domains in the dishevelled protein as well as novel suppressing alleles of axin. Genetics 161, 747-762.

Perez TD, Tamada M, Sheetz MP, James Nelson WJ (2008) Immediate-early signaling induced by E-cadherin engagement and adhesion. Journal of Biological Chemistry, 283, 5014-5022.

Peters-Van Der Sanden MJH, Kirby ML, Gittenberger-De Groot A, Tibboel D, Mulder MP, Meijers C (1993) Ablation of various regions within the avian vagal neural crest has differential effects on ganglion formation in the fore-, mid- and hindgut. Developmental Dynamics Vol. 196, Issue 3, pages 183-194. 
Pilot F, Philippe JM, Lemmers C, Lecuit T (2006). Spatial control of actin organization at adherens junctions by the synaptotagmin-like protein Btsz. Nature 442, 580-584.

Price MA (2006) CKI, there's more than one: caseine kinase I family members in Wnt and Hedegehog signaling. Genes Dev. 20, 399-410.

Pukrop T, Gradl D, Henningfeld KA, Knochel W, Wedlich D, Kuhl M (2001) Identification of two regulatory elements within the high mobility group box transcription factor XTCF-4. Journal of Biological Chemistry 276(12): 8968-78.

Qian D, Jones C, Rzadzinska A, Mark S, Zhang X, Steel KP, Dai X, Chen P (2007) Wnt5a functions in planar cell polarity regulation in mice. Dev. Biol. 306:121-133. doi:10.1016/j.ydbio.2007.03.01.

Ragland JW, Raible DW (2004) Signals derived from the underlying mesoderm are dispensable for zebrafish neural crest induction. Dev. Biol. 276(1):16-30.

Ransom DG, Hens MD, DeSimone DW (1993) Integrin Expression in Early Amphibian Embryos: cDNA Cloning and Characterization of Xenopus $\beta_{1}, \beta_{2}, \beta_{3}$, and $\beta_{6}$ Subunits. Developmental Biology Vol. 160 (1) 265-275.

Rao TP, Kühl M (2010) An updated overview on Wnt signaling pathways: a prelude for more. Circ Res.,106(12):1798-806.

Ratcliffe MJ, Itoh K, Sokol SY (2000) A positive role for the PP2A catalytic subunit in Wnt signal transduction. J Biol Chem 275: 35680-35683.

Rauch GJ, Hammerschmidt M, Blader P, Schauerte HE, Strahle U, Ingham PW, McMahon AP, Haffter P (1997). Wnt5 is required for tail formation in the zebrafish embryo. Cold Spring Harbor Symp. Quant. Biol. 62, 227-234.

Raven CP, Kloos J (1945) Induction by medial and lateral pieces of the archenteron roof, with special reference to the determination of the neural crest. Acta Neerl Morphol 5:348-362.

Ricklin D, Hajishengallis G, Yang K, Lambris JD (2010) Complement: A key system for immune surveillance and homeostasis. Nature Immunology, 11, 785-797.

Rida PC, Chen P (2009) Line up and listen: Planar cell polarity regulation in the mammalian inner ear. Seminars in Cell \& Developmental Biology, 20 pp. 978-985.

Ridley AJ, Hall A (1992) The small GTP-binding protein rho regulates the assembly of focal adhesions and actin stress fibers in response to growth factors. Cell 70(3):389-399. 
Ridley AJ, Paterson HF, Johnston CL, Diekmann D, Hall A (1992) The small GTPbinding protein rac regulates growth factor-induced membrane ruffling. Cell 70(3):401-10.

Ridley AJ. Life at the leading edge (2011) Cell 145:1012-1022.

Riesgo-Escovar JR, Jenni M, Fritz A, Hafen E (1996) The Drosophila Jun-N-terminal kinase is required for cell morphogenesis but not for DJun-dependent cell fate specification in the eye. Genes Dev. 10, 2759-2768.

Ro S, Hwang SJ, Muto M, Jewett WK, Spencer NJ (2006) Anatomic modifications in the enteric nervous system of piebald mice and physiological consequences to colonic motor activity. Am. J. Physiol. Gastrointest. Liver Physiol. 290, G710-G718.

Robinson V, Smith A, Flenniken AM, Wilkinson DG (1997) Roles of Eph receptors and ephrins in neural crest pathfinding. Cell Tissue Res. 290, 265-274.

Roël G1, van den Broek O, Spieker N, Peterson-Maduro J, Destrée O (2003) Tcf-1 expression during Xenopus development. Gene Expr Patterns.,3(2):123-6.

Rogers CD, Saxena A, Bronner ME (2013) Sip1 mediates an E-cadherin-to-Ncadherin switch during cranial neural crest EMT. J. Cell Biol. 203, 835-847.

Rohatgi R, Nollau P, Ho HYH, Kirschner MW, Mayer BJ (2001) Nck and phosphatidylinositol 4,5-bisphosphate synergistically activate actin polymerization through the N-WASP-Arp2/3 pathway. J. Biol.Chem. 276, 26448-26452.

Roose J, Molenaar M, Peterson J, Hurenkamp J, Brantjes $\mathrm{H}$, Moerer $\mathrm{P}$, van deWetering M, DestreeO, Clevers H (1998) The Xenopus Wnt effector XTcf-3 interacts with Groucho-related transcriptional repressors. Nature 395: 608-612.

Rothbächer U, Laurent MN, Deardorff MA, Klein PS, Cho KWY, Fraser SE (2000) Dishevelled phosphorylation, subcellular localization and homomerization regulate its role in early embryogenesis. EMBO J. 19, 1010-1022.

Sadaghiani B, Thiebaud C (1987) Neural crest development in the Xenopus laevis embryo, studied by interspecific transplantation and scanning electron microscopy. Dev.Biol. 124, 91-110.

Saint-Jeannet JP, He X, Varmus HE, Dawid IB (1997) Regulation of dorsal fate in the neuraxis by Wnt-1 and Wnt-3a. Proc Natl Acad Sci USA 94,13713-13718.

Santiago A, Erickson CA (2002) Ephrin-B ligands play a dual role in the control of neural crest cell migration. Development 129, 3621-3632. 
Sato A, Khadka DK, Liu W, Bharti R, Runnels LW, Dawid IB, Habas R (2006) Profilin is an effector for Daam1 in non-canonical Wnt signaling and is required for vertebrate gastrulation. Development 133, 4219-4231.

Sauka-Spengler T, Bronner-Fraser M (2008) A gene regulatory network orchestrates neural crest formation Nat. Rev. Mol. Cell Biol., 9, pp. 557-568.

Savin T, Doyle PS (2005) Static and dynamic errors in particle tracking microrheology. Biophysical journal 88(1): 623-38.

Schambony A, Wedlich D (2007) Wnt-5A/Ror2 regulate expression of XPAPC through an alternative noncanonical signaling pathway. Dev. Cell 12, 779-792.

Schlessinger K, McManus EJ, Hall A (2007) Cdc42 and noncanonical Wnt signal transduction pathways cooperate to promote cell polarity. J. Cell Biol. 178:355-361.

Seeling JM, Miller JR, Gil R, Moon RT, White R, Virshup DM (1999) Regulation of ßcatenin signaling by the B56 subunit of protein phosphatase 2A. Science 283: 208991

Segalen M, Bellaïche $Y$ (2009) Cell division orientation and planar cell polarity pathways. Semin Cell Dev Biol., 20(8):972-7.

Seifert JR, Mlodzik M (2007) Frizzled/PCP signalling: a conserved mechanism regulating cell polarity and directed motility. Nat Rev Genet. 8(2):126-38.

Selleck MA, Bronner-Fraser M (1995) Origins of the avian neural crest: the role of neural plate-epidermal interactions. Development 121:525-538.

Serafini T, Kennedy TE, Galko MJ, Mirzayan C, Jessell TM, Tessler-Lavigne M (1994) The Netrins Define a Family of Axon Outgrowth-Promoting Proteins Homologous to C. elegans UNC-6. Cell, Vol. 79, 409-424.

Serbedzija GN, Bronner-Fraser M, Fraser SE (1994) Developmental potential of trunk neural crest cells in the mouse. Development 120, 1709-1718.

Sheldahl LC, Slusarski DC, Pandur P, Miller JR, Kuhl M, Moon RT (2003) Dishevelled activates Ca2+ flux, PKC, and CamKII in vertebrate embryos. J. Cell Biol. 161, 769-777.

Shih W, Yamada, S (2012) N-cadherin-mediated cell-cell adhesion promotes cell migration in a three-dimensional matrix. Cell Adhesion \& Migration, 6, 513-517. 
Shimada Y, Usui T, Yanagawa S, Takeichi M, Uemura T (2001) Asymmetric colocalization of Flamingo, a seven-pass transmembrane cadherin, and Dishevelled in planar cell polarization. Curr. Biol. 11, 859-863.

Shimizu H, Julius MA, Giarré M, Zheng Z, Brown AM, Kitajewski J (1997) Transformation by Wnt family proteins correlates with regulation of beta-catenin. Cell Growth Differ.,8 (12):1349-58.

Shnitsar I, Borchers A. (2008) PTK7 recruits dsh to regulate neural crest migration. Development 135(24):4015-24.

Shook D, Keller R (2003) Mechanisms, mechanics and function of epithelialmesenchymal transitions in early development. Mech. Dev. 120, 1351-1383.

Simões-Costa M, Bronner ME (2015) Establishing neural crest identity: a gene regulatory recipe. Development.,142(2):242-57.

Simrick S1, Massé K, Jones EA (2005) Developmental expression of Pod 1 in Xenopus laevis. Int J Dev Biol.,49(1):59-63.

Sluss HK, Han Z, Barrett T, Davis RJ, Ip YT (1996) A JNK signal transduction pathway that mediates morphogenesis and an immune response in Drosophila. Genes Dev. 10, 2745-2758.

Smith A, Robinson V, Patel K, Wilkinson DG (1997) The EphA4 and EphB1 receptor tyrosine kinases and ephrin-B2 ligand regulate targeted migration of branchial neural crest cells. Curr. Biol. 7, 561-570.

Smith KM, Gaultier A, Cousin H, Alfandari D, White JM, DeSimone DW (2002) The cysteine-rich domain regulates ADAM protease function in vivo. J. Cell Biol. 159, 893-902.

Smith WC, Harland RM (1991) Injected Xwnt-8 RNA acts early in Xenopus embryos to promote formation of a vegetal dorsalizing center. Cell 67(4): 753-65.

Smolich BD, McMahon JA, McMahon AP, Papkoff J (1993) Wnt family proteins are secreted and associated with the cell surface. Mol Biol Cell.,4(12):1267-75.

Soille, P (2003) Morphological image analysis: principles and applications. Berlin; London:Springer.

Song H, Goetze S, Bischof J, Spichiger-Haeusermann C, Kuster M, Brunner E, Basler K (2010) Coop functions as a corepressor of Pangolin and antagonizes Wingless signaling. Genes Dev 24: 881-886. 
Sprowl S, Waterman ML (2013) Past visits present: TCF/LEFs partner with ATFs for $\beta$-catenin-independent activity. PLoS Genet. 2013;9(8):e1003745.

Staal FJ, Clevers H (2000) Tcf/Lef transcription factors during T-cell development: unique and overlapping functions. Hematol J.,1(1):3-6.

Staal FJ, Luis TC, Tiemessen MM (2008) WNT signalling in the immune system: WNT is spreading its wings. Nat Rev Immunol.,8(8):581-93.

Stamos JL, Weis WI (2013) The $\beta$-catenin destruction complex. Cold Spring Harb Perspect Biol., 5(1):a007898.

Steventon B, Araya C, Linker C, Kuriyama S, Mayor R (2009) Differential requirements of BMP and Wnt signalling during gastrulation and neurulation define two steps in neural crest induction. Development. 136(5):771-9.

Steventon B, Carmona-Fontaine C, Mayor R (2005) Genetic network during neural crest induction: from cell specification to cell survival. Semin. Cell Dev. Biol., 16, pp. 647-654.

Strachan LR, Condic ML (2003) Neural crest motility and integrin regulation are distinct in cranial and trunk populations. Dev. Biol. 259:288-302.

Strachan LR, Condic ML (2008) Neural crest motility on fibronectin is regulated by integrin activation. Exp. Cell Res., 314, pp. 441-452.

Struhl G, Casal J, Lawrence PA (2012) Dissecting the molecular bridges that mediate the function of Frizzled in planar cell polarity. Development 139:3665-3674.

Strutt DI (2001) Asymmetric localization of frizzled and the establishment of cell polarity in the Drosophila wing. Mol. Cell 7, 367-375.

Strutt H, Strutt D (2008) Differential stability of flamingo protein complexes underlies the establishment of planar polarity. Curr. Biol. 18:1555-1564.

Stuhlmiller TJ, Garcia-Castro MI (2012) FGF/MAPK signaling is required in the gastrula epiblast for avian neural crest induction. Development 139(2):289-300.

Tada M, Kai M (2009) Noncanonical Wnt/PCP Signaling During Vertebrate Gastrulation. Zebrafish 6:29-40.

Tada M, Smith JC (2000) Xwnt11 is a target of Xenopus Brachyury: regulation of gastrulation movements via Dishevelled, but not through the canonical Wnt pathway. Development, 127(10):2227-38. 
Takada R, Satomi Y, Kurata T, Ueno N, Norioka S, Kondoh H, Takao T, Takada S (2006) Monounsaturated fatty acid modification of Wnt protein: Its role in Wnt secretion. Dev Cell 11: 791-801.

Takeuchi S, Takeda K, Oishi I, Nomi M, Ikeya M, Itoh K, Tamura S, Ueda T, Hatta T, Otani $\mathrm{H}$, et al. (2000) Mouse Ror2 receptor tyrosine kinase is required for the heart development and limb formation. Genes Cells 5:71-78.

Tamai K, Semenov M, Kato Y, Spokony R, Liu C, Katsuyama Y, Hess F, SaintJeannet JP, He X (2000) LDL-receptor-related proteins in Wnt signal transduction. Nature 407, 530-535.

Taneyhill LA, Coles EG, Bronner-Fraser M (2007) Snail2 directly represses cadherin6B during epithelial-to-mesenchymal transitions of the neural crest. Development 134, 1481-1490.

Taylor J, Abramova N, Charlton J, Adler PN (1998) Van Gogh: A new Drosophila tissue polarity gene. Genetics 150:199-210.

Theveneau E, Marchant L, Kuriyama S, Gull M, Moepps B, Parsons M, Mayor R (2010) Collective Chemotaxis Requires Contact-Dependent Cell Polarity. Developmental Cell 19, 39-53.

Theveneau E, Mayor R (2011a) Can mesenchymal cells undergo collective cell migration? The case of the neural crest. Cell Adh. Migr. 5

Theveneau E, Mayor R (2011b) Collective cell migration of the cephalic neural crest: The art of integrating information. Genesis, 49, 164-176.

Theveneau E, Mayor R (2012) Neural crest delamination and migration: From epithelium-to-mesenchyme transition to collective cell migration. Developmental Biology 366 (1), 34-54.

Theveneau E, Steventon B, Scarpa E, Garcia S, Trepat X, Streit A, Mayor R (2013) Chase-and-run between adjacent cell populations promotes directional collective migration. Nat. Cell Biol., 15, pp. 763-772.

Thiery JP, Acloque H, Ruby YJ, Huang M, Nieto A (2009) Epithelial-Mesenchymal Transitions in Development and Disease. Cell 139.

Thiery JP, Sleeman JP (2006) Complex networks orchestrate epithelialmesenchymal transitions. Nature Reviews, Molecular Cell Biology Vol. 7. 
Thiesen H, Purcell J, Bennett M, Kansagara D, Syed A, Marsh JL (1994) dishevelled is required during wingless signaling to establish both cell polarity and cell identity. Development 120:347-360.

Tobin JL, Di Franco M, Eichers E, May-Simera H, Garcia M, Yan J, Quinlan R, Justice MJ, Hennekam RC, Briscoe J, Tada M, Mayor R, Burns AJ, Lupski JR, Hammond P, Beales PL (2008) Inhibition of neural crest migration underlies craniofacial dysmorphology and Hirschsprung's disease in Bardet-Biedl syndrome. Proc. Natl. Acad. Sci. USA.

Toyofuku T, Yoshida J, Sugimoto T, Yamamoto M, Makino N, Takamatsu H, Takegahara N, Suto F, Hori M, Fujisawa H, Kumanogoh A, Kikutani H (2008) Repulsive and attractive semaphorins cooperate to direct the navigation of cardiac neural crest cells. Developmental Biology Vol. 321 (1), Pages 251-262.

Tree DR, Shulman JM, Rousset R, Scott MP, Gubb D, Axelrod JD (2002) Prickle mediates feedback amplification to generate asymmetric planar cell polarity signaling. Cell 109, 371-381.

Tribulo C, Aybar MJ, Nguyen VH, Mullins MC, Mayor R (2003) Regulation of Msx genes by a Bmp gradient is essential for neural crest specification. Development 130(26):6441-6452.

Tuttle AM, Hoffman TL, Schilling TF (2014) Rabconnectin-3a regulates vesicle endocytosis and canonical Wnt signaling in zebrafish neural crest migration. Plos Biology 12(5): e1001852.

Twigg SR, Kan R, Babbs C, Bochukova EG, Robertson SP, Wall SA, Morriss-Kay GM, Wilkie AO (2004) Mutations of ephrin-B1 (EFNB1), a marker of tissue boundary formation, cause craniofrontonasal syndrome. Proc. Natl Acad. Sci. USA 101, 86528657.

Usui T, Shima Y, Shimada Y, Hirano S, Burgess RW, Schwarz TL, Takeichi M, Uemura $T$ (1999) Flamingo, a seven-pass transmembrane cadherin, regulates planar cell polarity under the control of Frizzled. Cell 3;98(5):585-95.

Vallin J, Thuret R, Giacomello E, Faraldo MM, Thiery JP, Broders F (2001) Cloning and characterization of three Xenopus slug promoters reveal direct regulation by Lef/beta-catenin signaling. J. Biol. Chem. 276, 30350-30358.

van Amerongen R, Mikels A, Nusse R (2008) Alternative wnt signaling is initiated by distinct receptors. Science Signaling, 1, re9.

van Amerongen R, Nusse R (2009) Towards an integrated view of Wnt signaling in development. Development 136, 3205-3214. 
van de Wetering $M$, Cavallo $R$, Dooijes $D$, van Beest $M$, van Es J, Loureiro J, Ypma A, Hursh D, Jones T, Bejsovec A, Peifer M, Mortin M, Clevers H (1997) Armadillo coactivates transcription driven by the product of the Drosophila segment polarity gene dTCF. Cell. 88, 789-99.

van den Heuvel M, Harryman-Samos C, Klingensmith J, Perrimon N, Nusse R (1993) Mutations in the segment polarity genes wingless and porcupine impair secretion of the wingless protein. EMBO J., 12(13):5293-302.

Vasioukhin V, Bauer C, Yin M, Fuchs E (2000) Directed actin polymerization is the driving force for epithelial cell-cell adhesion. Cell 100:209-219.

Vinson CR, Conover S, Adler PN (1989) A Drosophila tissue polarity locus encodes a protein containing seven potential transmembrane domains. Nature 338: 263-264.

Vinson CR1, Adler PN (1987) Directional non-cell autonomy and the transmission of polarity information by the frizzled gene of Drosophila. Nature 14;329(6139):549-51.

Vladar EK, Bayly RD, Sangoram AM, Scott MP, Axelrod JD (2012) Microtubules enable the planar cell polarity of airway cilia. Curr Biol., 22(23):2203-12.

Wagner G, Peradziryi H, Wehner P, Borchers A (2010) PlexinA1 interacts with PTK7 and is required for neural crest migration. Biochemical and Biophysical Research Communications Vol 402 (2), Pages 402-407.

Wallingford JB, Habas R (2005) The developmental biology of Dishevelled: an enigmatic protein governing cell fate and cell polarity. Development, 132(20):442136.

Wallingford JB, Rowning BA, Vogeli KM, Rothbacher U, Fraser SE, Harland RM (2000) Dishevelled controls cell polarity during Xenopus gastrulation. Nature 405, 8185.

Wang H, Copeland NG, Gilbert DJ, Jenkins NA, Tessier-Lavigne M (1999) Netrin-3, a Mouse Homolog of Human NTN2L, Is Highly Expressed in Sensory Ganglia and Shows Differential Binding to Netrin Receptors. The Journal of Neuroscience, 19(12):4938-4947.

Wang HU, Anderson DJ (1997) Eph family transmembrane ligands can mediate repulsive guidance of trunk neural crest migration and motor axon outgrowth. Neuron 18, 383-396.

Wang Y, Macke JP, Abella BS, Andreasson K, Worley P, Gilbert DJ, Copeland NG, Jenkins NA, Nathans J (1996) A large family of putative transmembrane receptors 
homologous to the product of the Drosophila tissue polarity gene frizzled. J Biol Chem.,271(8):4468-76.

Wang Y, Nathans J (2007) Tissue/planar cell polarity in vertebrates: New insights and new questions Development, 134 pp. 647-658

Weinstein DC, Hemmati-Brivanlou A (1999) Neural induction. Annu Rev Cell Dev Biol 15:411-433.

Werner T, Hammer A, Wahlbuhl M, Bosl MR, Wegner M (2007) Multiple conserved regulatory elements with overlapping functions determine Sox10 expression in mouse embryogenesis. Nucleic Acids Res. 35, 6526-6538.

Whittaker CA, DeSimone DW (1993) Integrin subunit mRNAs are differentially expressed in early Xenopus embryos. Development 117, 1239-1249.

Wikramanayake AH, Peterson R, Chen J, Huang L, Bince JM, McClay DR, Klein WH (2004) Nuclear beta-catenin-dependent Wnt8 signaling in vegetal cells of the early sea urchin embryo regulates gastrulation and differentiation of endoderm and mesodermal cell lineages. Genesis; 39(3):194-205.

Wilkinson DG, Bhatt S, Chavrier P, Bravo R, Charnay P (1989) Segment-specific expression of a zinc-finger gene in the developing nervous system of the mouse. Nature 337:461-464.

Willert K1, Nusse R (2012) Wnt proteins. Cold Spring Harb Perspect Biol., 4(9):a007864.

Winning RS, Shea LJ, Marcus SJ, Sargent TD (1991) Developmental regulation of transcription factor AP-2 during Xenopus laevis embryogenesis. Nucleic Acids Res. 19,3709-3714.

Wodarz A, Nusse R (1998) Mechanisms of Wnt signaling in development. Annu Rev Cell Dev Biol. 14, 59-88.

Wolff T, Rubin GM (1998) Strabismus, a novel gene that regulates tissue polarity and cell fate decisions in Drosophila. Development 125:1149-1159.

Wong HC, Bourdelas A, Krauss A, Lee HJ, Shao Y, Wu D, Mlodzik M, Shi DL, Zheng $\mathrm{J}$ (2003) Direct binding of the PDZ domain of Dishevelled to a conserved internal sequence in the C-terminal region of Frizzled. Mol. Cell 12, 1251-1260.

Wu J, Yang J, Klein PS (2005) Neural crest induction by the canonical Wnt pathway can be dissociated from anterior-posterior neural patterning in Xenopus. Dev. Biol. 279(1):220-232. 
Wu MY, Ramel MC, Howell M, Hill CS (2011) SNW1 is a critical regulator of spatial BMP activity, neural plate border formation, and neural crest specification in vertebrate embryos. PLoS Biol 9(2):e1000593.

Yamamoto H, Hinoi T, Michiue T, Fukui A,Usui H, Janssens V, Van Hoof C, Goris J, Asashima M, Kikuchi A (2001) Inhibition of the Wnt signaling pathway by the PR61 subunit of protein phosphatase 2A. J Biol Chem 276: 26875-26882.

Yates LL, Dean CH (2011) Planar polarity: A new player in both lung development and disease. Organogenesis, 7 pp. 209-216

Yen WW, Williams M, Periasamy A, Conaway M, Burdsal C, Keller R, Lu X, Sutherland A (2009) PTK7 is essential for polarized cell motility and convergent extension during mouse gastrulation. Development 136(12):2039-48.

Yntema CL, Hammond WS (1954) The origin of intrinsic ganglia of trunk viscera from vagal neural crest in the chick embryo. J. Comp. Neurol. 101:515-541.

Yonemura S (2011) Cadherin-actin interactions at adherens junctions. Current Opinion in Cell Biology, 23, 515-522.

Yook JI, Li X-Y, Ota I, Hu C, Kim HS, Kim NH, Cha SY, Ryu JK, Choi YJ, Kim J et al. (2006) A Wnt-Axin2-GSK3beta cascade regulates Snail1 activity in breast cancer cells. Nat. Cell Biol. 8, 1398-1406.

Yoon SJ, Wills AE, Chuong E, Gupta R, Baker JC (2011) HEB and E2A function as SMAD/FOXH1 cofactors. Genes Dev.,25(15):1654-61.

Yost C, Torres M, Miller JR, Huang E, Kimelman D, Moon RT (1996) The axisinducing activity, stability, and subcellular distribution of b-catenin is regulated in Xenopus embryos by glycogen synthase kinase 3. Genes Dev 10:1443-1454

Young CS, Kitamura M, Hardy S, Kitajewski J (1998) Wnt-1 Induces Growth, Cytosolic $\beta$-Catenin, and Tcf/Lef Transcriptional Activation in Rat-1 Fibroblasts. Mol Cell Biol., 18(5): 2474-2485.

Yu HH, Kolodkin AL (1999) Semaphorin signaling: a little less per-plexin. Neuron 22, 11-14.

Yu HH, Moens CB (2005) Semaphorin signaling guides cranial neural crest cell migration in zebrafish. Dev. Biol. 280, 373-385.

Zallen JA (2007) Planar polarity and tissue morphogenesis.Cell, 129 pp. 1051-1063 
Zandy N, Playford M, Pendergast NM (2007) Abl tyrosine kinases regulate cell-cell adhesion through Rho GTPases. Proceedings of the National Academy of Sciences of the United States of America, 104, 17686-17691.

Zeng X, Tamai K, Doble B, Li S, Huang H, Habas R, Okamura H, Woodgett J, He X (2005) A dual-kinase mechanism for Wnt co-receptor phosphorylation and activation. Nature. 438, 873-7.

Zhang D, Ighaniyan S, Stathopoulos L, Rollo B, Landman K, Hutson J, Newgreen D (2014) The neural crest: a versatile organ system. Birth Defects Res. C. Embryo Today. 


\section{Curriculum vitae}

\section{Educational and professional activities}

\subsubsection{1 - 30.09.2015}

PhD thesis under the supervision of Prof. Dr. Annette Borchers: Controlled levels of canonical Wnt signaling are required for neural crest migration" Georg-AugustUniversität in Göttingen, Germany, Philipps-Universität in Marburg Germany

20.09.2010 - 15.07.2011

Molecular Biology MSc/PhD Program of Georg-August-Universität and International Max Planck Research School in Göttingen, Germany

$01.10 .2008-02.07 .2010$

Intercollegiate Faculty of Biotechnology, University of Gdańsk and Medical University of Gdańsk in Gdańsk, Poland - obtained a Master degree in the field of molecular virology

$01.10 .2005-02.07 .2008$

Faculty of Biology, University of Gdańsk in Gdańsk, Poland - obtained a Bachelor degree in the field of molecular biology

\section{Awards/Scholarships}

28th May 2015

Poster Prize awarded during Wnt Symposium May 26-28, 2015 in Heidelberg in Memoriam of Herbert Steinbeisser entitled Mechanisms; functions and evolution of Wnt-signaling pathways.

5th October 2009/ 7th January 2011

Congratulatory letters form Dean of Intercollegiate Faculty of Biotechnology, University of Gdańsk and Medical University of Gdańsk in Gdańsk on outstanding results in academic years: 2008/2009 - 2009-2010 and active participation in the academic community life, which have contributed to the promotion and strengthening of Faculty's high position. 
$2010-2011$

Stipend of the Excellence Foundation for the Promotion of the Max Planck Society

\section{Abroad Experience}

30 August - 30 September 2009

Training at Wuhan Institute of Virology, Chinese Academy of Science. Participation in project Molecular Characterization of Nonstructural Protein NS38 of Grass Carp Reovirus Ling Shao, Chao Fan, Ewa Maj, and Qin Fang VIROLOGICA SINICA, April 2010, 25 (2):123-129

\section{Additional skills}

Polish language- native speaker English language- proficiency in speaking and writing German language- intermediate in speaking and writing European driving license- $B$ category 